\title{
A Measurement of the Analyzing Power for Pion Proton Elastic Scattering Between 471 and $687 \mathrm{MeV} / \mathrm{C}$
}

\author{
Alireza Mokhtari-Amirmajdi*
}

DISCLAIMER \begin{abstract}
This report was prepared as an account of work sponsored by an agency of the United States
Government. Neither the United States Government nor any agency therecf, nor any of their bility for the accuracy, completeness, or usefulness of assumes any legal liability or responsiprocess disclosed, or represents that its use would of any information, apparatus, product, or ence herein to any specific commercial product, process infringe privately owned rights. Fiefermanufacturer, or otherwise does not necessarily constitute orvice by trade name, trademark, menjation, or favoring by the Uniter States Govertitute or imply its endorsement, recomand opinions of authors expressed herein do not necessarily any agency thereof. The views United States Government or any agency thereof.
\end{abstract}

-Guest Sciantlst at Los Alamos. Department of Physics, University of California at Los Angeles, CA 90024. 
List of Tables

I. Pion-Nucleon Scattering Formalism

1.l. Spin 0-Spin 1/2 Elastic Scattering

II. The Experiment

2.1. The Pion Beam

2.1.1 Beam Monitoring

2.2. The Target

2.2.1 The Polarized Proton Target Magnet 80

$\begin{array}{ll}2.2 .2{ }^{4} \mathrm{He} \text { System } & 80\end{array}$

$\begin{array}{ll}2.2 .3{ }^{3} \mathrm{He} \text { System } & 84\end{array}$

2.2 .4 Cryostat Vacuum $\quad 88$

2.2.5 The rmometry $\quad 88$

2.2.6 Polarized Target Material 90

2.2.7 Microwaves 90

2.2.8 NMR 91

2.3. Detectors and Detection System 93

2.3.1 The LAS 94

2.3.2 The Recoil Detector 100

2.4. The Electronics 103

2.4.1 The LAS Electronic Circuit 103

2.4.2 The Recoil Detector Electronic Circuit 105

2.4.3 The Inhibit Electronic Circuit 108

2.4.4 The Coincidence Event Trigger Electronic Circuit 110

2.5. Data Acquisition

III. The Analysis

3.1 Scatlering Data Analysis

114

3.2 The Target Polarization Ana -ysis 130

3.3 The Background 
3.4 Statistical Uncertainties

3.5 Results and Conclusions

Acknowledgements

186

References

187

Appendix A. Beam Line Shunt Readings

190 
2.1. Summary of beam characteristics

2.2. Beam Elements for the LAS 97

2.3. The LAS wire chamber dimensions 98

3.1. A representative set of efficiencies 129

3.2. Target polarization as a function of time 133

$3.3(\varepsilon)-(h)$. Background to signal ratio 141

$3.4(a)-(b)$. A consistency check on the data 146

3.5(a). $A_{N}\left(\pi^{+} p \rightarrow \pi^{+} p\right)$ at $471 \mathrm{MeV} / \mathrm{c} \quad 159$

3.5(b). $A_{N}\left(\pi^{+} p \rightarrow \pi^{+} p\right)$ at $471 \mathrm{MeV} / \mathrm{c} \quad 160$

3.6(a). $A_{N}\left(\pi_{p}^{+} \rightarrow \pi^{+} p\right)$ at $547 \mathrm{MeV} / \mathrm{c} \quad 161$

3.6(b). $A_{N}\left(i^{+}{ }^{+} \rightarrow i^{+} p\right)$ at $547 \mathrm{MeV} / \mathrm{c} \quad 162$

3.7(a). $A_{N}^{N}\left(\pi^{+}{ }^{+}+\pi^{+} p\right)$ at $625 \mathrm{MeV} / c \quad 163$

3.7(b). $A_{N}\left(r^{+} p+\pi^{+}+\right.$p $)$at $625 \mathrm{MeV} / \mathrm{c} \quad 164$

3.8(a). $\mathrm{A}_{\mathrm{N}}\left(\mathrm{ir}^{+} \mathrm{p} \cdot \pi^{+} \mathrm{p}\right)$ at $687 \mathrm{MeV} / \mathrm{c} \quad 165$

3.8(b). $\mathrm{A}_{\mathrm{N}}\left(\right.$ ir $\left.{ }_{-}^{+} \mathrm{p}+\mathrm{if}_{-}^{+} \mathrm{p}\right)$ at $687 \mathrm{MeV} / \mathrm{c} \quad 166$

$3.9(a) \cdot A_{N}(\pi-p \rightarrow \pi-p)$ at $471 \mathrm{MeV} / \mathrm{c} \quad 167$

3.9(b). $A_{N}\left(\pi^{-} p+\pi p\right)$ at $471 \mathrm{MeV} / \mathrm{c} \quad 168$

$3.10(a) \cdot A_{N}\left(\pi_{-}^{-} p+\pi_{-}^{-} p\right)$ at $547 \mathrm{MeV} / \mathrm{c} \quad 169$

$3.10(\mathrm{~b}) \cdot \mathrm{A}_{\mathrm{N}}\left(\mathrm{ir}+\mathrm{H}^{-} \mathrm{p}\right)$ at $547 \mathrm{MeV} / \mathrm{c} \quad 170$

3.11(a). $A_{N}\left(\pi^{-} p+\pi^{-p}\right)$ at $625 \mathrm{MeV} / \mathrm{c} \quad 171$

$3.11(b) \cdot A_{N}\left(\right.$ ir $\left.p \rightarrow \pi_{-}^{-p}\right)$ at $625 \mathrm{MeV} / \mathrm{c} \quad 172$

$3.12(a) \cdot A_{N}\left(\pi^{-p} \rightarrow \pi^{-} p\right)$ at $687 \mathrm{MeV} / \mathrm{c} \quad 173$

3.12(b). $A_{N}(\pi p \rightarrow \pi p)$ at $687 \mathrm{MeV} / \mathrm{c} \quad 174$ 


\section{List of Figures}

Figure

Page

1.1. Polar coordinates of $\vec{k}_{f} \quad 14$

1.2. Symmetry operations applied to a scattering experiment 21

1.3. Scattering to the left and right

1.4. Projectile helicity frame and recoll proton laboratory helicity frame 36

1.5. The component of polarization to be measured to determine the spin projection rotation angle $B$

1.6. The amplitudes $a^{+}, a^{-}$, and $a^{0}$ form a triangle in the complex plane

2.l. Time structure of the LAMPF $\mathrm{H}^{+}$beam

55

2.2. Los Alamos Meson Physics Facility

63

2.3. Beam switchyard and experimental area A 66

2.4. The Pion Particle Physics channel 68

2.5. Layout of the experimental setup 70

2.6. Elements of the polarized proton target 75

2.7. Energy levels of an isolated electron-proton pair in a strong magnetic field with and without dipole-dipole interactions

2.8. PTM-Ricardo field maps

2.9. Field maps of polarized target magnet 82

2.10. The separator

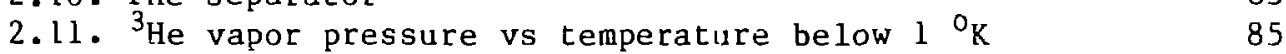

2.12. Target cryostat and target insert 86

2.13. Temperature versus resistance curve for an Allen Bradley $100 \Omega, 1 / 2$ watt resistor

2.14. Positive polarization absorbs energy from RF field. Negative polarization gives energy to RF field 91

2.15. LAS and its particle detection systems 95

2.16. Recoil detector

2.17. Electronic schematic for the LAS

2.18. Electronic diagram for recoil detector

2.i9. Electronic schematic for dead time generator

2.20. Electronic schematic for the coincidence event

3.1. Bending of charged particles in the PTM

3.2. $\pi^{ \pm}$p elastic scattering in the presence of the PTi 117

3.3. The LAS time of flight

3.4. The LAS front scintillator pulse height

3.5. Horizontal and vertical target projections

3.6. Deviations of the tracks from the central ray between the third and fourth chambers in the LAS

3.7. Recoil time of flight

3.8. Recoil pulse helght

3.9. The LAS momentum spectrum

3.10. A typical thermal equilibrium spectrum 
3.11. Regions of the LAS momentum spectrum used for

3.12. The difference between polarization up and

polarization down yields, $Y \uparrow-Y \downarrow$, and $Y \uparrow+Y \downarrow-2 B$

3.13. Normalized background vs $\theta$

3.14. $A_{N}\left(\pi^{+} p \rightarrow \pi^{+} p\right)$ at $471 \mathrm{MeV} / c^{c}$

3.15. $A_{N}^{N}\left(\pi^{+} p \rightarrow \pi^{+} p\right)$ at $547 \mathrm{MeV} / \mathrm{c}$

175

3.16. $A_{N}^{N}\left(\pi^{+} p+\pi_{+}^{+} p\right)$ at $625 \mathrm{MeV} / \mathrm{c}$

3.17. $\mathrm{A}_{\mathrm{N}}^{\mathrm{N}}\left(\pi^{+} \mathrm{P} \rightarrow \pi^{+} \mathrm{p}\right)$ at $687 \mathrm{MeV} / \mathrm{c}$

3.18. $A_{N}^{N}\left(\pi^{-p}+\pi^{-p}\right)$ at $471 \mathrm{MeV} / \mathrm{c}$

3.19. A $A_{N}^{N}(\pi-p \rightarrow \pi-p)$ at $547 \mathrm{MeV} / \mathrm{c}$

3.20. $A_{N}^{N}\left(\pi^{-} \mathrm{p} \rightarrow \pi^{-} \mathrm{p}\right)$ at $625 \mathrm{MeV} / \mathrm{c}$

180

3.21. $A_{N}^{N}\left(\pi^{-} p+\pi^{-p}\right)$ at $687 \mathrm{MeV} / \mathrm{c}$

181

3.22. Results of the present experiment compared to Martin et al. data

3.23. Results of the present experiment compared to Abaev et al. data

183

184

3.24. Results of the present experiment compared to Bekreuvi et al. data 
ABSTRACT OF THE DISSERTATION

A Measurement of the Analyzing Power

for Pion Proton Eiastic Scattering

Between 471 and $687 \mathrm{MeV} / \mathrm{c}$

by

\author{
Alireza Mokhtari-Amirmajdi \\ Doctor of Philosophy in Physics \\ University of Calffornia, Los Angeles, 1984 \\ Professor Bernard M. K. Nefkens, Chatr
}

The analyzing power, $A_{N}$, has been measured for $\pi^{ \pm} p \rightarrow \pi{ }^{ \pm}$at 471 , 547, 625, and $687 \mathrm{MeV} / \mathrm{c}$ in an angular range corresponding to $-0.9<\cos \theta_{\mathrm{cm}}<0.8$. A polarized proton target with polarization axis normal to the scattering plane was used. The scattered pion and recoll proton were detected in coincidence, using a magnetic spectrometer and a wire chamber/scintillator array, except in cases where one of the particles was kinematically inaccessible. Statistical uncertaintles in the data are as low as 0.02 ; systematic uncertainties are estimated to be less than $3 \%$. The $\pi^{-}$p data are characterized by large values of analyzing power, and rapid variations in the angular distribution with incident momentum. The measurements are compared with the results of existing partial wave analysis. 
Introduction and Motivation

Around 1968, electron scattering experiments at SLAC gave the first clear hints that pointlike particles existed inside the proton.l Earlier, 1n 1964, Gell-Mann ${ }^{2}$ and Zweig $^{3}$ had proposed that the protons, neutrons and all other strongly interacting particles - the hadrons are made from basic ertities named "quarks", a heretofore unobserved particles. Over the last ten years, this proposal has become firmly accepted even though quarks have not been observed directly. More recent research has found that fruces between quarks are extremely simple and that these forces explai, why free quarks can not be seen. Therefore, we can not first determine to properties of isolated quarks and then predict the properties of compuste states. Nevertheless we can still learn much indirectly. The quark model enables one to make sense out of the multitude of baryon and meson resonances that have been found. When interpreted as excited states of multi-quark (QQQ)

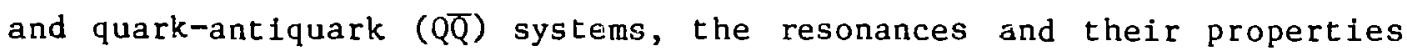
may be understood. It is known from atomic physics that spectroscopy can reveal not only the constituents of a system, but aiso the nature of the forces between the constituents. Experimentally, the rich 
spectroscopy of the non-strange baryons, are known quite well up to masses of about $2 \mathrm{GeV}$. Spectroscopy j.s concerned with the study of how particles absorb and emit energy, in particular with the properties of individual levels such as energy, angular momentum, isospin, electric and magnetic moments, transition rates and so forth. Scatteriug data are used to extract the properties and wavefunctions of lavels and thus contribute to theories for predicting them. When a model is confronted with experimental results, the first step is usually to lock at the spectra of energy levels of known spin and parity. Thus, determining the energies, spins and parities of the quantum states is probably the most important aspect of spectroscopy.

The basic theory of hadrons, Quantum Chromodynamics (QCD), is a generalization of Quantum Electrodynamics (QED) - the theory of photons and electrons that underlies atomic physics. Instead of the electron and photon, QCD has the quark and gluon. The gluons should have odd spin, since even spin field quanta couple in the same way to particles and antiparticles, and hence would lead to unwanted $Q Q$ states degenerate with the $\bar{Q}$ mesons. The simplest possibility is $J=1$. While electrons come in only one electric charge state, quarks come in three color charge states. Emlssion or absorption of a photon leaves an electron as an electron, but emission or absorption of a gluon causes a change of the color state of quarks. Thus, the crucial consequence of the generalization of QED is that gluons, unlike photons, must carry color charge to insure color conservation. By assuming that color is associated with an SU(3) symmetry group, called $S U(3)_{C}$, one can explain 
why $Q Q Q$ and $Q \bar{Q}$ states exist, but $Q Q$ and $Q Q \bar{Q}$, for example, do not. If quarks belong to a triplet, and gluons to an octet repre,entation or SU(3) $C$, the SU(3) $C^{-i n v a r i a n t, ~ o n e-g l u o n ~ e x c h a n g e ~ f o r c e ~ d e t w e e n ~ q u a r k s ~}$ will result in color singlet states $Q Q Q$ and $Q \bar{Q}$ having lcwe: energy than any color non-singlet state. This theory: triplets of colored quarks interacting in an $S U(3) \mathrm{C}^{-i n v a r i a n t}$ way by the exchange of octets of colored gluons together with renormalizability or "asymptotic freedom" (the quark-quark interaction becomes feeble at short distances), is QCD. Therefore one can simply say that QCD is a sort of generalized QED, in which the eight gluons play a role analogous to that of the single photon, and in which the guage-invariance of QED is generalized to the non-Abelian gauge invariance involving the color group $\mathrm{SU}(3)_{\mathrm{C}}$. Three quarks bound together make up a baryon, a bound state of a quark and an antiquark constitutes a meson. From the many hundreds of hadrons now known, there are no established states that do not fit into either the QQQ or $Q \bar{Q}$ family. However, just as a world without charged matter would still have photons, one without quarks would sti.ll have a spectrum of gluons, and we expect the spectrum to be very rich in structure because QCD predicts confinement of the gluons. Theie is one phenomenon $i_{1}$ systems governed by the color force that has no equivalent in electromagnetic systems. It is a particle composed exclusively of gluons, bound together by their color charge. The analogous electromagnetic entity would be a bound state of two photons, but such a system can not exist because the photon has no charge. The gluon bound states are called glueballs. Thus, confinement leads to the belief that hadrons of pure glue should exist. One of the 
distinguishing characteristics of glueballs might be the spectrum of angular momentum states. Because the gluons have a spin of 1 , the glueball state of lowest energy is expected to be an S state with a total angular momentum of zero or two. Such states are predicted in many models. Various models differ in fine details but all agree that the lightest glueballs will have $\mathrm{J}^{\mathrm{PC}}=0^{++}, 0^{-+}, 2^{++}$and masses between 1 and $2 \mathrm{GeV}$. These models also indicate the existence of other simple color singlet systems. These are hybrid or hermaphrodite states which contain both constituent quarks and gluons: $Q \bar{Q} G$ and $Q^{3} G$, in which both quark and gluonic degrees of freedom are excited simultaneously.

If a particle is stable, its mass can be determined with perfect accuracy. If its lifetime is very brief, measurements of its mass will give a distribution with a width related to the value of the lifetime. For a normal strongly decaying hadron the width of the mass distribution would be equivalent to an energy range of about 100-150 MeV. In pion-nucleon collisions at lower energies one can detect the formation of short-lived intermediate states or resonances, whose width is of the order of $100 \mathrm{MeV}$, and whose spin and parity is determined by partial wave analysis of elastic and charge exchange (CEX) scattering or, possibly, through other reactions. A resonance accurs when a meta-stable state is formed. These states are not essentially different from the states stable under the strong interaction (such as the nucleon), in the same way that the ground state of an atom is not different in nature from an excited state. At incident pion energies below about $3 \mathrm{GeV}$, the $\pi \mathrm{N}$ system exhibits many 
resonant states. Most of these are only revealed by phase shift analysis of the differential cross-sections and polarization parameters. In the absence of a dynamical model for $\pi N$ scattering, the phenomenological analysis of low-to-medium energy $\pi N$ scattering has usually taken the form of partial wave analysis, which is practically the only method of obtaining $\pi N$ amplitudes and investigating resonances. Experimental data of high quality is very essential ror such an analysis. These resonances are gcanted the status of elementary particles ard at the same time thay be regarded in some sense as excited states of the nucleons so chat the determination of the energy levels, widths, spins, parities, and other quantum numbers may give important indications to the classification and symmetries of elementary particles and even to their structure. The study of the energy levels is known as hadron spectroscopy.

The ground state Su(3) multiplets are the $\mathrm{J}^{\mathrm{P}}=1 / 2^{+}$nucleon octet and $J^{P}=3 / 2^{+}$decuplet. These are the spin $s=1 / 2$ and $s=3 / 2$ configurations of three quarks with zero orbital angular momentum $\ell$. The spectrum of nucleon resonances is reasonably well established largely from $\pi N$ elastic scattering up to gbout a mass of $2100 \mathrm{MeV}$. Present theories are guided largely by the mass values. From 1500 to $1700 \mathrm{MeV}$, there is a band of aegative parity states with $\mathrm{J}^{\mathrm{P}}=1 / 2^{-}, 3 / 2^{-}, 5 / 2^{-}$. From 1700 to $1950 \mathrm{MeV}$, there is $a$ band of positive parity states with $\mathrm{J}^{\mathrm{P}}=\mathrm{I} / 2^{+}$to $7 / 2^{+}$. And from 1900 to $2100 \mathrm{MeV}$, there are several established negative parity states with $\mathrm{J}^{\text {P }}$ uf to $9 / 2^{-}$. This pattern of alternating parity and steadily increasing 
$J$ is characteristic of an $\&$ excitation spectrum. It is by now firmly established that the grcup symetry is $S U(6) \times 0(3)$, i.e. three spin $1 / 2^{+}$ quarks combined with angular momentum $\ell$. Combining the fundamental representations of three quirk flavors SU(3) with SU(2) of spin of the quarks one can form six-dimensional fundamental representation of SU(6). Combining three fundamental representations of SU(6) yields

$$
6 \times 6 \times 6=56+70+70+20 \text {. }
$$

Placing the quarks in a potential, e.g. a harmonic oscillator, in this

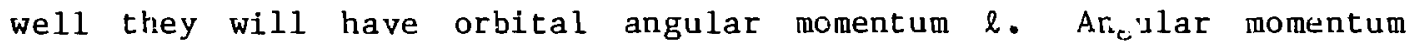
conservation emerges from rotational invariance in three dimensional space, the resulting group structure being $O(3)$. The full symmetry group structure of the quacks in a potential is then $\operatorname{SU}(6) \times 0(3)$. The most extensive quantitative model with this group structure is that of Isgur and Karl.4 The predictions of this model are in acceptable quantitative agreement with experimental data. The first excitation is the $[70, \ell=1]^{-}$. It 1 ies below $1 \mathrm{GeV} / \mathrm{c}$ in pion laboratory momentum. A crucial feature of the model is that it predicts that the first positive parity excitation consists of $[56,2]^{+},[56,0]^{+},[70,0]^{+}$, $[70,2]^{+}$, and $[20,1]^{+}$multiplets; the $[56,0]^{+}$and $[70,0]^{+}$are r.zdial excitations of the ground state. The $[56,2]^{+}$is almost completely established, one $[56,0]^{+}$is established, and there are signs of a second. The $\mathrm{SU}(6) \times 0(3)$ character of the spectrum is reasonably well established, although the symmetry is obviously badly broken in the mass values. A major open question below beam momenta of $2 \mathrm{GeV} / \mathrm{c}$ is whether the $[70,0]^{+},[70,2]^{+}$, and $[20,1]^{+}$multiplets, which are a 
crucial prediction of the model of Isgur and Karl, exist. The present evidence for any states belonging to these multiplets is tenuous. There are no good candidates for the missing multiplets. It is remarkable and worrying that such extensive multiplets should have escaped detection almost completely. So it is clear that in the 1-2 GeV/c momentum range we are still ignorant not only of the quantitative features of the spectrum, but also its qualitative features.

This experiment is the third of a complete set of measurements of TN scattering observables in the energy region of the first two resonances: $\Lambda(1232) \mathrm{P}_{33}$ and $\mathrm{N}(1440) \mathrm{P}_{11}$. The primary motivation for these measurements is to provide precise and unambiguous $\pi N$ amplitudes (or phase shifts). The importance of these measurements has been emphasized in several recent pub: cations. The charge exchange (CEX) data is more extensive and includes other energies than the elastic data. The partial wave analysis must use some model to evaluate the elastic and CEX data at the same energy for an energy Independent fit or de an energy dependent fit. Presently there are several partial wave solutions ${ }^{5-8}$ over our energy range which differ in the number and position of the resonances. Evilence of a new $P_{33}$ resonance has been found 5,6 , but the cited values of this resonance mass are quite different: ${ }^{5}(1522 \pm 13) \mathrm{MeV}$ and ${ }^{6}(1600 \pm 50) \mathrm{MeV}$. As another example the masses of $\Delta^{++}$and $\Delta^{0}$ have been determined as ${ }^{7} \mathrm{~m}_{\Delta}++1230.9 \pm 0.3 \mathrm{MeV}$ and $\mathrm{m}_{\Delta} \mathrm{j}=1233.6 \pm 0.5 \mathrm{MeV}$. This gives a mass splitting $\mathrm{m}_{\Delta} \mathrm{o} \mathrm{m}_{\Delta}++=2.7 \pm 0.6 \mathrm{MeV}$, while another partial wave analysis ${ }^{8}$ predicts $\mathrm{m}_{\Delta}{ }^{0-\mathrm{m}_{\Delta}}++=-0.9 \pm 0.4 \mathrm{Mev}$. 
Yet another partial wave analysis ${ }^{9}$ shows evidence for a spilt Roper $\left(P_{11}\right)$ resonance (which has the same quantum numbers as the nucleon), with a mass difference about $10 \mathrm{C} \mathrm{MeV}$. The first indication of the $P_{11}(1440)$ resonance splitting into two resonances came frum the Saclay phase shift analysis. 10

G. Karl et al. 11 have carried out a study of three-quark one-gluon configurations in the zero strangeness, positive parity sector. The $Q^{3} G$ spectrum performs just about as well in predicting masses as does the radially-excited oscillato: model. The Roper resonance is reproduced well. One is faced with the cholce of accepting as valid either the radially-excited $Q^{3}$ model or the $Q^{3} G$ ground state configuration, or perhaps both. We can consider three possibilities:

(a) Only radial excitations; $Q^{3} G$ is much higher in mass.

(b) Only $Q^{3} \mathrm{G}$; radial excitations are much higher in mass.

(c) Both radial excitations and $Q^{3} G$ are low in mass.

The last possibility is very unlikely, because we would then need two low Roper resonances and four $\mathrm{N} 5 / 2^{+}$states.

The CEX results together with the elastic scattering differential cross section and analyzing power measurements can be used in a model independent test of isospin invariance through the triangle inequality which will be discussed in section 1.4. As a test of the triangle ineguality the Leningrad group ${ }^{12}$ has analyzed existing data, including their $\mathrm{A}_{\mathrm{N}}\left(\pi^{-} \mathrm{P}+\pi^{-} \mathrm{P}\right)$; and they have found, at backward angles, a violation at the level of many standard deviations. To establish unambiguously 
the number of resonances and to decermine more precisely their characteristics new experimenta1 data are necessary.

Finally a few comments concerning the nomenciature of polarization measurements are in order. Scattering experiments in which the spin polarization of one of the particles is known or measured fali 1nto two classes :

(i) $\mathrm{A}(\mathrm{b}, \vec{c}) \mathrm{D}$ are polarization type experiments,

(ii) $A(\vec{b}, c) D$ are analyzing power type experiments.

The particle whose spin polarization is known or measured is denoted by an arrow. Experiments in which the spin polarization of two of reactants are measured are:

(iii) $A(\vec{b}, \vec{c}) D$ are polarization transfer type experiments,

(iv) $\vec{A}(\vec{b}, c) D$ are spin correlation (initial channei) cype experiments, (v) $A(b, \vec{c}) \vec{D}$ are spin correlation (final channel) type experiments. 


\section{CHAPTER ONE}

\section{PION-NUCLEON SCATTERING FORMALISM}

\subsection{Spin $0-$ Spin $1 / 2$ Elastic Scattering}

The scattering formalism of spin zero by spin one-half particles includes pion-nucleon scattering. To take account of the fact that the proton spin can flip in the collision, the asymptotic form of the scattered wave in the center of mass system can be written as a spinor with components

$$
\left|\psi_{\mu}\right\rangle=e^{i k z}\left|x_{\mu}\right\rangle+\frac{e^{i k r}}{r} M_{\nu \mu}\left|x_{\nu}\right\rangle
$$

The first term on the right-hand side represents the incoming plane wave, and we have assumed that the direction of the momentum is paralle1 to the z-axis. $\left|x_{\mu}\right\rangle,\left|x_{\nu}\right\rangle(\mu, \nu= \pm 1 / 2)$ denote the spin state of the incident and scattered particles, respectively. The $2 \times 2$ matrix $M$ acting in a two dimensional suin space relates the outgoing spinor to the incident one. The scattering amplitudes are the matrix elements 
of $M$. The second term in (1.1) represents the outgoing wave, and can be written as

$$
M_{\nu \mu}\left|x_{\nu}\right\rangle=a(\theta)\left|x_{\mu}\right\rangle+b(\theta, \phi)\left|x_{-\mu}\right\rangle
$$

The square of the amplitude $a(\theta)$ represents the probability that the pion in the final state moves away in the direction described by the polar angle $\theta$ relative to the incident direction (the z-axis) with the proton spin in the same direction as before the scattering, while the square of $b(\theta, \phi)$ represents the probability that the pion is scattered at angle $\theta$ and that the spin of the proton has been flipped. These two quantities are referred to as the "non spin-flip amplitude" and "spin-flip amplitude", respectively. The $2 \times 2$ matrix $M$ acts on the Initial nucleon spinor to produce the scattered state

$$
\left|x_{f}\right\rangle=M\left|x_{i}\right\rangle \text {, }
$$

where 1 and $\bar{i}$ derote "Initial" and "final", respectively.

The unit $2 \times 2$ matrix

$$
I=\left(\begin{array}{ll}
1 & 0 \\
0 & 1
\end{array}\right),
$$

and the three Pauli spin matrices

$$
\sigma_{1}=\left(\begin{array}{ll}
0 & 1 \\
1 & 0
\end{array}\right), \sigma_{2}=\left(\begin{array}{rr}
0 & -i \\
i & 0
\end{array}\right), \sigma_{3}=\left(\begin{array}{rr}
1 & 0 \\
0 & -1
\end{array}\right),
$$

form a complete set of $2 \times 2$ matrices. We may therafore write the $2 \times 2$ matrix $M$ as a linear combination of them

$$
M=a_{0} I+a_{1} \sigma_{1}+a_{2} \sigma_{2}+a_{3} \sigma_{3}=a_{0} I+\sum_{i=1}^{3} a_{i} \sigma_{1},
$$

where the coefficients $a_{0}, a_{1}, a_{2}, a_{3}$ are complex numbers. Angular momentum conservation requires the matrix elements of $M$ to be scalars, invariant under rotations. Since $M$ is a scalar the coefficients $a_{i}$ must be the components of a vector $\vec{a}$ and $\sum a_{i} \sigma_{1}(=\vec{a} \cdot \vec{\sigma})$ is a scalar and 
therefore invariant under rotations. There are only two Independent vertors at our disposal: $\vec{k}_{1}$ and $\vec{k}_{f}$. From these we can construct

$$
\hat{n}=\frac{\vec{k}_{i} \times \vec{k}_{f}}{\left|\vec{k}_{i} \times \vec{k}_{f}\right|},
$$

where $\vec{k}_{1}$ and $\vec{k}_{f}$ are the initial and final momenta, respectively, and $\hat{n}$ is the unit vector normal to the scattering plane (conforming to the Basel convention). The most general form of $M$ compatible with rotational invariance $1 \mathrm{~s}^{13}$

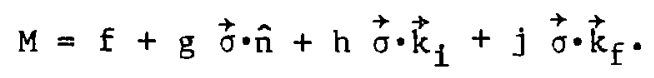

If time reversal invariance holds, $M$ must be unchanged under the . substitutions $\vec{k}_{1} \rightarrow-\vec{k}_{f}, \vec{k}_{f} \rightarrow-\vec{k}_{1}$ and $\vec{\sigma} \rightarrow-\vec{\sigma}$, hence $h=j$. Under the parity operation, $\vec{k}_{i} \rightarrow-\vec{k}_{i}, \vec{k}_{f} \rightarrow-\vec{k}_{f}$, and $\vec{\sigma} \rightarrow \vec{\sigma}$. If parity is conserved, the pseudoscalars $\vec{\sigma}_{\vec{k}} \vec{k}_{i}$ and $\stackrel{\vec{\sigma} \cdot \vec{k}_{f}}{ }$ are excluded, and $\mathrm{h}$ and $j$ can not appear at all. In this case, time reversal is less restrictive than parity invariance and does not impose ary additional limitations on the $M$ matrlx. Note that this condition is not necessarily true in all cases. The most general form of M invariant under parity operation is

$$
M=f(\theta)+g(\theta) \vec{\sigma} \cdot \hat{n}, *
$$

where $f$ and $g$ are two complex parameters. The determination of the matrix $M$ apart from an overall phase requires three measurements. Since $M$ is a function of the scattering angle, these measurements must be done at each angle. A complete set of measurements include in this case a determination of the unpolarized cross section, the left-right

Fifferent authors use differept forms for M. For example, Sakurail 14 uses $M=f(\theta)+i g(\theta) \vec{\sigma} \cdot \hat{n}$, 
asymmetry in the scattering from a transversely polarized target, and an investigation of the polarization of the recoil protons following scattering from polarized (in the scattering plane) proton targets. Measurement of the asymetry of elastic scattering by a transverse polarized target is equivalent, owing to the polarization-asymmetry equality, to a determination of the polarizaiton of the recoil particle in experiments with an unpolarized target. But, in general, the data rate is much higher for asymetry measurements.

The first term in eq. (1.6) can not induce any changes in the spin state of the particles, while the second term may. Let us now investigate the physical meaning of the $\vec{\sigma} \cdot \hat{n}$ term in this equation. The $f$ and $g$ amplitudes depend on the scattering angle and the energy. Choosing the z-axis as the direction of the incident particles, we have

$$
\begin{aligned}
& \hat{k}_{i}=(0,0,1), \\
& \hat{k}_{f}=(\sin \theta \cos \phi, \sin \theta \sin \phi, \cos \theta),
\end{aligned}
$$

where $\theta$ and $\phi$ are polar and azimuthal angles of $\vec{k}_{f}$ (see figire 1.1), and

$$
\hat{\mathrm{n}}=(-\sin \phi, \cos \phi, 0) \text {. }
$$

Consider the scattering to be in the $x-z$ plane; then $\phi=0, \hat{n}$ is along

\footnotetext{
* The Polarization-asymmetry equality in spin $0-\operatorname{spin} 1 / 2$ scattering is a result of invariance under spatial rotations and parity. In the case of spin $1 / 2$ scattering from a target of spin $s \neq 0$ the polarization-asymmetry equality does not follow from parity invariance, it follows from time reversal invariance. Wolfenstein and Ashkin 16 and Dalitz ${ }^{17}$ originally derived this equality for the spin $1 / 2-\operatorname{spin} 1 / 2$ case by examining the most general transition operator invariant under rotations, parity and time reversal. Bell and Mand ${ }^{18}$ pointed out that it holds independent of parity invariance.
} 


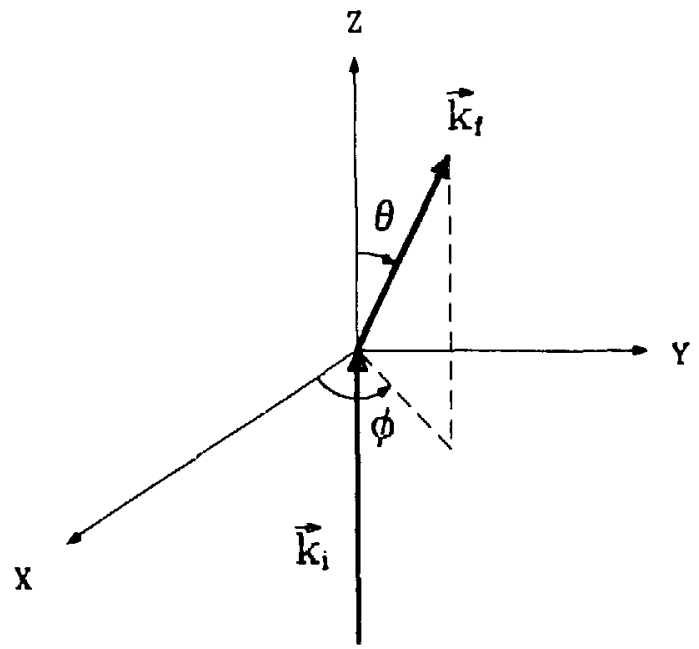

Figure 1.1 Polar angles of $\vec{k}_{f}$.

the +y direction and $g(\theta) \vec{\sigma} \cdot \hat{n}=g(\theta) \sigma_{y}$. If the quantization axis is along the incident beam direction (z-axis), then

$$
\sigma_{x}=\left(\begin{array}{ll}
0 & 1 \\
1 & 0
\end{array}\right), \sigma_{y}=\left(\begin{array}{cc}
0 & -1 \\
i & 0
\end{array}\right), \sigma_{z}=\left(\begin{array}{rr}
1 & 0 \\
0 & -1
\end{array}\right),
$$

and

$$
M=f(\theta)+g(\theta) \sigma_{y}=\left(\begin{array}{rr}
f & -i g \\
i g & f
\end{array}\right)
$$

Under the scattering $|+\rangle \rightarrow M|+\rangle$ and $|\rightarrow \therefore M| \rightarrow$ where $|+\rangle$ and $\mid \rightarrow$ are two component spinors corresponding to the nucleon spin along the +2 and $-z$ direction, respectively

$$
\begin{aligned}
& |+\rangle+M|+\rangle=E|+\rangle+i g \mid \rightarrow, \\
& |\rightarrow+M| \rightarrow=-i g|+\rangle+f|-\rangle .
\end{aligned}
$$

As can be seen, $g(\theta)$ describes spin-flip scattering. Hence the differential cross secticn fir scattering without spin-flip is given by

$$
\begin{aligned}
& \frac{d \sigma}{d \Omega}(|+\rangle+|+\rangle)=|\langle+|M|+\rangle|^{2}=|f|^{2}, \\
& \frac{d \sigma}{d \Omega}(|-\rangle+|-\rangle)=|\langle-|M|-\rangle|^{2}=|f|^{2},
\end{aligned}
$$


while the spin-flip differential cross section is

$$
\begin{aligned}
& \frac{\mathrm{d} \sigma}{\mathrm{d} \Omega}(|+\rangle+|-\rangle)=|\langle-|M|+\rangle|^{2}=|+i g|^{2}=|g|^{2}, \\
& \frac{\mathrm{d} \sigma}{\mathrm{d} \Omega}(|-\rangle+|+\rangle)=\mid\left\langle+t_{1}, i^{2}=|-i g|^{2}=|g|^{2} .\right.
\end{aligned}
$$

Note that in this case the cross sections (1.8) and (1.9) do not exhibit a left-right asymetry. If the axis of quantization is along ac $x$-axis

$$
\sigma_{x}=\left(\begin{array}{cc}
1 & 0 \\
0 & -1
\end{array}\right), \quad \sigma_{y}=\left(\begin{array}{ll}
0 & 1 \\
1 & 0
\end{array}\right), \quad \sigma_{z}=\left(\begin{array}{rr}
0 & -i \\
\mathbf{i} & 0
\end{array}\right),
$$

and the scattering matrix becomes

$$
\mathrm{M}=\left(\begin{array}{ll}
\mathrm{f} & \mathrm{g} \\
\mathrm{g} & \mathrm{f}
\end{array}\right),
$$

leading to the same cross sections (1.8) and (1.9). If the representation of $\vec{\sigma}$ is chosen such that the quantization is along the $y$-axis, then

$$
\sigma_{x}=\left(\begin{array}{rr}
0 & -i \\
i & 0
\end{array}\right), \quad \sigma_{y}=\left(\begin{array}{rr}
1 & 0 \\
0 & -1
\end{array}\right), \quad \sigma_{z}=\left(\begin{array}{ll}
0 & 1 \\
1 & 0
\end{array}\right),
$$

and

$$
M=\left(\begin{array}{cc}
f+g & 0 \\
0 & f-g
\end{array}\right)
$$

Therefore

$$
\begin{aligned}
& |+\rangle+M|+\rangle=(f+g)|+\rangle, \\
& |\rightarrow \rightarrow M| \rightarrow\rangle=(f-g) \mid \rightarrow,
\end{aligned}
$$

there is no spin-flip. Thus the scattering is different for different initial spin states, which explicitly exhibits that scattering is spin-dependent if $g \neq 0$. If the initial polarization lies in the scattering plane, as a result of scattering it may flip, but there is no left-right asymmetry. If the initial polarization is normal to the scattering plane there will be no spin flip, but there is left-right as ymmetry. 
To describe the results of a polarization experiment, one must choose a coordinate system for the projectile polarization and another one for the outgoing particle polarization. These coordinate systems may be identical, or chosen to be different. For the projectile the frame with the z-axis along its momentum $\vec{k}_{i}, y$ along the normal to the scattering plane $\vec{k}_{i} \times \vec{k}_{f}$, and $x$ such as to form a right-handed coordinate system is used. This frame is called the projectile helicity frame. For the scattered particles the $x^{-}, y^{-}, z^{-}$coordinate system is defined with the $z^{\prime}$-axis along its momentum $\vec{k}_{f}, y^{\prime}$ along $\vec{k}_{1} \times \vec{k}_{f}$, and $x^{-}$is chosen to form a right-handed system. In this case, it must be specified whether $\vec{k}_{f}$ represents the scattered particle momentum in the laboratory or in the center of mass system. If $\vec{k}_{f}(\mathrm{~cm})$ is used, the corresponding coordinate system is referred to as the outgoing helicity frame. If $\vec{k}_{f}(1 \mathrm{ab})$ is used, the corresponding system is referred to as the outgoing laboratory helicity frame. For the projectile we will use the projectile helicity frame. A polarimeter measures the polarization components with respect to the laboratory direction of motion, so we will use the outgoing laboratory helicity frame for scattered particles.

Let us now express the differential sross section and polarization for $\pi-N$ scattering in terms of the amplitudes and initial polarization. We will assume at first that the incident particles are fully polarized. A fully polarized spinor in the direction $\theta_{i}, \phi_{i}$ could be obtained as follows. We start with the epin state pointing in the $+z$-direction: $|+\rangle$ and rotate it about the $y$-axis by an angle $\theta_{i}$, then 
rotate 1 about the $z$-axis by $\phi_{i}$

$$
\begin{aligned}
\left|x_{i}\right\rangle & =e^{-i \cdot \sigma_{z} \phi_{1} / 2} e^{-i \sigma_{y} \theta_{1} / 2}|+\rangle \\
& =\left(\begin{array}{l}
\cos \left(\theta_{i} / 2\right) e^{-i \phi_{1} / 2} \\
\sin \left(\theta_{1} / 2\right) e^{+i \phi_{1} / 2}
\end{array}\right) .
\end{aligned}
$$

If the plane of scattering is at an azlmuthal angle $\phi$, then the normal to the scattering plane is given by eq. (1.7), so that

$$
\tilde{\sigma} \cdot \hat{n}=\left(\begin{array}{cc}
0 & -i e^{-1 \phi} \\
i e^{1 \phi} & 0
\end{array}\right) .
$$

The scattering matrix is given by eq. (1.6), or explicitly

$$
M=\left(\begin{array}{cc}
f & -i g e^{-i \phi} \\
1 g e^{1 \phi} & f
\end{array}\right) .
$$

The differential cross section for scattering from the inttial state $\left|x_{i}\right\rangle$ to the final state $\left|x_{f}\right\rangle$ can be calculated from the scattering amplitude

$$
\begin{aligned}
\frac{d \sigma}{d \Omega} & =\left|\left\langle x_{f}|M| x_{i}\right\rangle\right|^{2} \\
& =\left\langle x_{i}|M t| x_{f}\right\rangle\left\langle x_{f}|M| x_{i}\right\rangle .
\end{aligned}
$$

If we do not measure the spin orientation in the final state, the differential cross section for elastic scattering from a given initial spin orientation is

$$
\begin{aligned}
\frac{\mathrm{d} \sigma}{\mathrm{d} \Omega} & =\sum_{X_{f}}\left\langle x_{i}|M \dagger| x_{f}\right\rangle\left\langle x_{f}|M| x_{i}\right\rangle \\
& =\left\langle x_{i}\left|M{ }^{\dagger}\right| x_{i}\right\rangle .
\end{aligned}
$$

We can write this equation in a slightly different way:

$$
\frac{\mathrm{d} \sigma}{\mathrm{d} \Omega}=\left\langle\mathrm{Mx}_{i} \mid M x_{i}\right\rangle \text {. }
$$

Using eqs. $(1.10)$ and $(1.12)$ we find

$$
\begin{aligned}
\left|M x_{i}\right\rangle & =\left(\begin{array}{cc}
f & -i g e^{-1 \phi}
\end{array}\right)\left(\begin{array}{l}
\cos \left(\theta_{i} / 2\right) e^{-1 \phi_{i} / 2} \\
\sin \left(\theta_{i} / 2\right) e^{+i \phi_{1} / 2}
\end{array}\right) \\
& =\left(\begin{array}{l}
f \cos \left(\theta_{i} / 2\right) e^{-1 \phi_{1} / 2}-i g \sin \left(\theta_{1} / 2\right) e^{-i\left(\phi-\phi_{i} / 2\right)} \\
f \sin \left(\theta_{1} / 2\right) e^{+i \phi_{i} / 2}+i g \cos \left(\theta_{i} / 2\right) e^{+i\left(\phi-\phi_{i} / 2\right)}
\end{array}\right) .
\end{aligned}
$$


Substituting $(1.15)$ into $(1.14)$ the differential cross section becomes

$$
\frac{\mathrm{d} \sigma}{\mathrm{d} \Omega}=|\mathrm{f}|^{2}+|\mathrm{g}|^{2}-\sin \theta_{1} \sin \left(\phi-\phi_{1}\right)\left(\mathrm{fg}{ }^{*}+\mathrm{f}^{*} \mathrm{~g}\right) \text {. }
$$

The inttal polartzation is defined as

$$
\overrightarrow{\mathrm{e}}_{i}=\frac{\left\langle x_{i}|\vec{\sigma}| x_{i}\right\rangle}{\left\langle x_{i} \mid x_{i}\right\rangle}
$$

where $\left|x_{i}\right\rangle$ is given by eq. (1.10), and is normalized

$$
\left\langle x_{i} \mid x_{i}\right\rangle=1
$$

From eqs. (1.10) and $(1.11)$ we have

$$
\begin{aligned}
\vec{P}_{i} \cdot \hat{n} & =\left\langle\chi_{i}|\vec{\sigma} \cdot \hat{n}| x_{i}\right\rangle \\
& =\sin \theta_{1} \sin \left(\phi_{1}-\phi\right) .
\end{aligned}
$$

Substituting eq. (1.18) into $(1.16)$ we find

$$
\begin{aligned}
\frac{\mathrm{d} \sigma}{\mathrm{d} \Omega} & =|\mathrm{f}|^{2}+|\mathrm{g}|^{2}+\left(\mathrm{fg}{ }^{*}+\mathrm{f}^{*} \mathrm{~g}\right) \stackrel{+}{\mathrm{P}}_{\mathrm{i}} \cdot \hat{\mathrm{n}} \\
& =|\mathrm{f}|^{2}+|\mathrm{g}|^{2}+2 \operatorname{Re}\left(\mathrm{fg}{ }^{*}\right) \overrightarrow{\mathrm{P}}_{\mathrm{i}} \cdot \hat{\mathrm{n}} .
\end{aligned}
$$

Aiternatively, substituting eq. (1.6) into (1.13) we Find

$$
\begin{aligned}
& \frac{d \sigma}{d \Omega}=\left\langle x_{1}\right|\left(f+g \vec{\sigma} \cdot \hat{n}: \dagger(f+g \vec{\sigma} \cdot \hat{n})\left|x_{1}\right\rangle\right. \\
& =\left\langle x_{i}\left|\left(t^{*}+g^{*} \vec{\sigma} \cdot \hat{n}\right)(f+g \vec{\sigma} \cdot \hat{n})\right| x_{i}\right\rangle \\
& =\left\langle x_{i}\left|\left(|f|^{2}+f^{*} g \vec{\sigma} \cdot \hat{n}+f_{g}{ }^{*} \cdot \hat{\sigma}+|g|^{2}(\vec{\sigma} \cdot \hat{n})(\vec{\sigma} \cdot \hat{n})\right)\right| x_{i}\right\rangle .
\end{aligned}
$$

Nothing that

$$
\begin{gathered}
\left\langle\left. x_{i}|| f\right|^{2} \mid x_{i}\right\rangle=|f|^{2}, \\
\left\langle x_{j}\left|f^{*} g \vec{\sigma} \cdot \hat{n}\right| x_{i}\right\rangle=f^{*}{ }_{g}\left\langle x_{i}|\vec{\sigma}| x_{i}\right\rangle \cdot \hat{n}
\end{gathered}
$$

and using the initial polarization definition given by eq. (1.17):

$$
\begin{aligned}
& \left\langle x_{i}\left|f^{*} g \vec{\sigma} \cdot \hat{n}\right| x_{i}\right\rangle=f^{*} \overrightarrow{g P}_{i} \cdot \hat{n}, \\
& \left\langle x_{i}\left|f g^{*+} \cdot \hat{n}\right| x_{i}\right\rangle=\mathrm{fg}^{*+} P_{i} \cdot \hat{n} .
\end{aligned}
$$

Using the identity

$$
(\vec{\sigma} \cdot \vec{A})(\vec{\sigma} \cdot \vec{B})=\vec{A} \cdot \vec{B}+i \vec{A} \times \vec{B} \cdot \vec{\sigma}
$$


we find

$$
(\vec{\sigma} \cdot \hat{n})(\vec{\sigma} \cdot \hat{n})=1 \text {, }
$$

therefore

$$
\left\langle\left. x_{i}|| g\right|^{2}(\vec{\sigma} \cdot \hat{n})(\vec{\sigma} \cdot \hat{n}) \mid x_{i}\right\rangle=|g|^{2} .
$$

Putting everything together:

$$
\frac{\mathrm{d} \sigma}{\mathrm{d} \Omega}=|\mathrm{f}|^{2}+|\mathrm{g}|^{2}+\left(\mathrm{fg}{ }^{*}+\mathrm{f}^{*} \mathrm{~g}\right) \overrightarrow{\mathrm{P}}_{\mathrm{i}} \cdot \hat{\mathrm{n}}
$$

which is the same as eq. (1.19), as expected.

We see that in general the differential cross section depends not only on $k$ and $\theta$, but also on the spin orientation of the incident particles (more exactly, on the angle between the normal $\hat{\mathfrak{n}}$ to the scattering plane and the direction of initial polarization $\overrightarrow{\mathrm{P}}_{1}$ ). It is seen that the spin component normal to $\hat{\mathbf{n}}$ contributes nothing to the scattering cross section. We will see later that the spin-flip amplitude $g(\theta)$, is proportional to $\sin \theta$. Thus, unless $\operatorname{Re}\left(\mathrm{fg}^{*}\right)=0$, the scattering exhibit a left-right asymetry. This asymetry in the scattering from a polarized target is a consequence of the nucleon spin and it is important in studies of scattering for noncentral forces.

If the system is completely unpolarized in the initial state we have $\left|\vec{P}_{i}\right|=0$, and when we average eq. (1.19) over the initial spin orientations, the corresponding cross section is

$$
\left(\frac{d \sigma}{d \Omega}\right)_{\text {unpol }}=|f(\theta)|^{2}+|g(\theta)|^{2}
$$

Thus for an unpolarized target the differential cross section does not depend on the azimuthal angle $\phi$. 
The polarization of the scattered particles $\overrightarrow{\mathrm{P}}_{\mathrm{f}}$ may be expressed in terms of the initial polarization vector $\overrightarrow{\mathrm{P}}_{\mathfrak{i}}$. By definition

$$
\overrightarrow{\mathrm{P}}_{\mathrm{f}}=\frac{\left\langle x_{\mathrm{f}}|\vec{\sigma}| x_{\mathrm{f}}\right\rangle}{\left\langle x_{\mathrm{f}} \mid x_{\mathrm{f}}\right\rangle} .
$$

The final state $\left|x_{f}\right\rangle$ is related to the initial state $\left|x_{i}\right\rangle$ through the scattering matrix: $\left|x_{f}\right\rangle=M\left|x_{i}\right\rangle \cdot\left|x_{i}\right\rangle$ and $\left|x_{f}\right\rangle$ may be defined in different ccordinate systems. The scattered particle polarization becomes

$$
\begin{aligned}
\overrightarrow{\mathrm{P}}_{\mathrm{f}} & =\frac{\left\langle M x_{i}|\overrightarrow{\mathrm{C}}| M x_{i}\right\rangle}{\left\langle M x_{i} \mid M x_{i}\right\rangle} \\
& =\frac{\left\langle x_{i}\left|M^{\dagger} \vec{\sigma} M\right| x_{i}\right\rangle}{\left\langle x_{i}\left|M^{\dagger} M\right| x_{i}\right\rangle} .
\end{aligned}
$$

Using ( 1.6$)$ for $M$ we find

$$
\left\langle x_{i}\left|M^{\dagger}+M\right| x_{1}\right\rangle=\left\langle x_{i}\left|\left(f^{*}+g^{*}+\vec{\sigma} \cdot \hat{n}\right) \stackrel{\sigma}{\sigma}(f+g \stackrel{\sigma}{\sigma} \cdot \hat{n})\right| x_{i}\right\rangle .
$$

With the help of the identities

$$
\begin{aligned}
& \vec{\sigma}(\vec{\sigma} \cdot \hat{n})=\hat{n}+i \hat{n} \times \vec{\sigma}, \\
& (\vec{\sigma} \cdot \hat{n}) \vec{\sigma}=\hat{n}-i \hat{n} \times \vec{\sigma}, \\
& (\vec{\sigma} \cdot \hat{n}) \vec{\sigma}(\vec{\sigma} \cdot \hat{n})=2 \hat{n}(\vec{\sigma} \cdot \hat{n})-\vec{\sigma},
\end{aligned}
$$

and the definition of $\overrightarrow{\mathrm{P}}_{i}$ given by eq. $(1.17),(1.23)$ becomes

$$
\left\langle\chi_{i}\left|M^{\dagger}+\vec{\sigma}\right| x_{i}\right\rangle=\left(|f|^{2}-|g|^{2}\right) \vec{P}_{i}+\left(f g^{*}+f^{\star} g+2|g|^{2} \stackrel{P}{P}_{i} \cdot \hat{n}\right) \hat{n}+i\left(f g^{*}-f^{*} g\right) \vec{P}_{i} \times \hat{n} \text {. }
$$

Substituting (1.24) into (1.22) and noting that

$$
\left\langle x_{1}\left|M^{\dagger} M\right| x_{i}\right\rangle=\frac{d \sigma}{d \Omega}
$$

where $\frac{d \sigma}{d \Omega}$ is given by $(1.19)$, we find

$$
\stackrel{\vec{P}}{f}_{f}=\frac{\left(|f|^{2}-|g|^{2}\right) \overrightarrow{\mathrm{P}}_{1}+\left(f g^{*}+f^{*} g+2|g|^{2} \vec{P}_{i} \cdot \hat{n}\right) \hat{n}+1\left(f g^{*}-f^{*} g\right) \vec{P}_{i} \times \hat{n}}{|f|^{2}+|g|^{2}+\left(f g^{*}+f^{*} g\right) \vec{P}_{i} \cdot \hat{n}} .
$$

The general structure of $\overrightarrow{\mathrm{P}}_{\mathrm{f}}$ is what would be expected, since there are 
only the axial vectors $\hat{\mathrm{n}}, \overrightarrow{\mathrm{P}}_{i}$, and $\overrightarrow{\mathrm{P}}_{i} \times \hat{\mathrm{n}}$ upon which the axial vector $\overrightarrow{\mathrm{P}}_{f}$ can possibly depend. If the incident beam is unpolarized, we have $\left|\overrightarrow{\mathrm{P}}_{i}\right|=0$, so that

$$
\overrightarrow{\mathrm{P}}_{\mathrm{f}}=\frac{\mathrm{fg}^{*}+\mathrm{f}^{*} \mathrm{~g}}{|\mathrm{f}|^{2}+|\mathrm{g}|^{2}} \hat{\mathbf{n}} .
$$

The scattered beam is polarized along $\hat{n}$, $i$.e. perpendicular to the scattering plane and is dependent upon the interference between the two parts of the scattered wave. Large polarizations can result when the interference term is comparable to the cross section Itself.

Paricy nonconserving terms in the scattering amplitude will produce components of $\overrightarrow{\mathrm{P}}_{\mathrm{f}}$ in the scattering plane. This can be understood in terms of a simple geometrical argument. Consider the scattering experiment depicted in figure 1.2 (a). The $y$-axis is

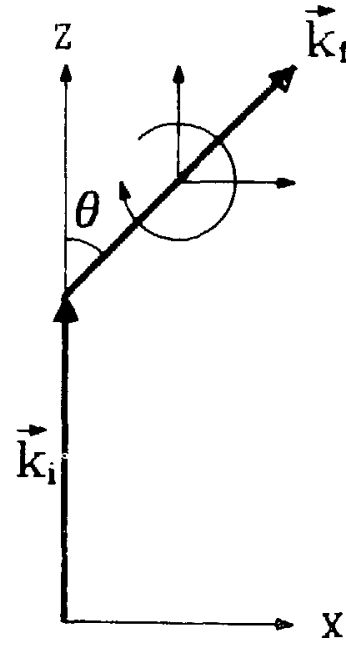

(a)

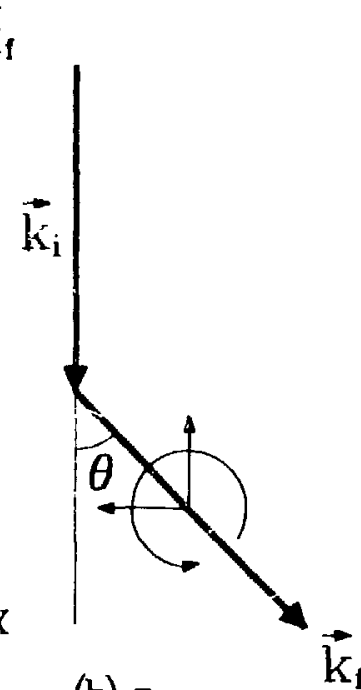

(b) $z \rightarrow-z$

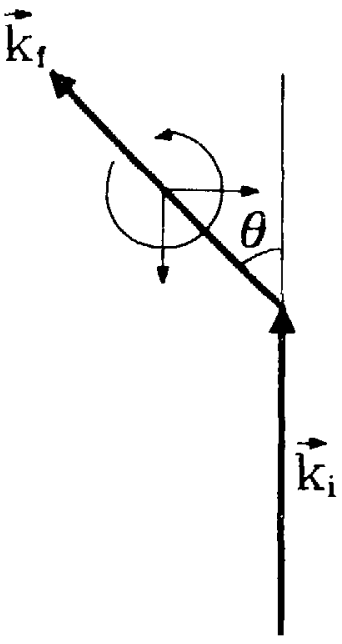

(c) $\pi$ about $\mathbf{x}$

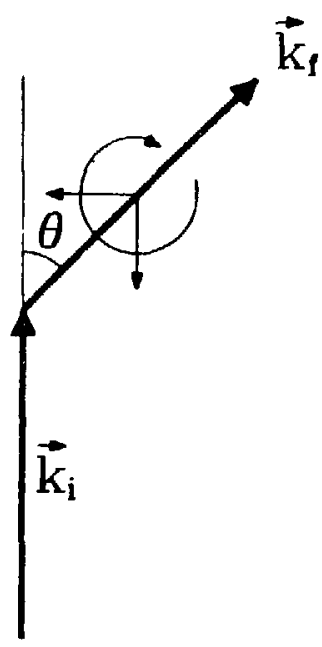

(d) $\pi$ about $z$

Figure 1.2. Symmetry operations applied to a scattering experiment. 
pointing into the page, the unmarked arrows are components of $\overrightarrow{\mathrm{P}}_{f}$ in the scattering plane, and the circular arrow gives the sense of $\stackrel{+}{\mathrm{P}}_{f}$ along the y-axis. $W$ then períorm the following sequ tce of operations: ( 1 ) a reflection in the $x-y$ plane; (if) a rotation through $\pi$ about the $x$-axis; (iii) a rotation through $\pi$ about the $z$-axis. Polarization is the average value of spin operator and under reflection behaves like orbital angular momentum. The reflection in the $x-y$ plane produces the following transformations of the orbital angular momentum:

$$
\begin{aligned}
& R_{x y} L_{x} R_{x y}^{-1}=-L_{x}, \\
& R_{x y} L_{y} R_{x y}^{-1}=-L_{y}, \\
& R_{x y} L_{z} R_{x y}^{-1}=+L_{z},
\end{aligned}
$$

where $R_{x y}$ is the unitary operator associated with this reflection. These relations must hold for any angular momentum operator. Thus in going frow figure 1.2 (a) to figure 1.2 (b)

$$
\begin{aligned}
& P_{f}^{x}+-P_{f}^{x}, \\
& P_{f}^{y}+-P_{f}^{y}, \\
& P_{f}^{z} \rightarrow+P_{f}^{z} .
\end{aligned}
$$

We observe that the components of $\overrightarrow{\mathrm{P}}_{\mathrm{f}}$ in the scattering plane have changed sign in going from (a) to (d), but that its component normal to the scattering plane has not. Hence $\stackrel{\vec{P}}{f}_{\mathrm{f}}$ can not have any components in the scattering plane. $\overrightarrow{\mathrm{P}}_{\mathrm{f}}$ must lie along $\hat{\mathrm{n}}$ when parity is conserved. If reflection and rotation invariance holds, the probabilities for the collision processes shown in these four figures must be equal. Comparing (c) and (d) we conrlude that the probability of scattering to the right through $\theta$ and finding $\overrightarrow{\mathrm{P}}_{\mathrm{f}}$ up must equal that for scattering through the same $\dot{c}^{\prime}$ le to the left and finding $\overrightarrow{\mathrm{P}}_{\mathrm{f}}$ down. 
So far we have assumed that the initial state is completely polartzed (or completely unpolarized). In the next section we will show that a wave function (or spinor) corresponds to a fully polarized state. To generalize the discussion to partially polarized inftial states we need to use the density matrix formalism. 


\subsection{Polarization of Spin 1/2 Particles}

To describe the orientation of a particle with nonvanishing spin s, we introduce a polarization vector $\vec{P}$ by the definition

$$
\overrightarrow{\mathrm{P}}=\frac{1}{\mathrm{~s}}\langle\overrightarrow{\mathrm{S}}\rangle \text {. }
$$

$\vec{P}$ is proportional to the expectation value of the spin operator $\vec{S} ;$ it "points" in the direction of the particle's spin.

For spin $1 / 2$ particles, the polarization vector $\overrightarrow{\mathrm{P}}$ is the expectation value of the Pauli spin vector $\vec{a}$ in the normalized state $|x\rangle$ :

$$
\overrightarrow{\mathrm{P}}=\langle\vec{\sigma}\rangle \text {. }
$$

The magnitude of $\vec{P}$ is called the degree of polarization; it is given by

$$
|\vec{P}|=\sqrt{\left\langle\sigma_{x}\right\rangle^{2}+\left\langle\sigma_{y}\right\rangle^{2}+\left\langle\sigma_{z}\right\rangle^{2}} \text {. }
$$

For a normalized spinor $|x\rangle$ :

$$
|x\rangle=\left(\begin{array}{l}
a \\
b
\end{array}\right) ; \quad a^{*} a+b^{*} b=1 \text {. }
$$

Straightforward computation gives

$$
\begin{aligned}
& \left\langle\sigma_{x}\right\rangle=a b^{*}+a^{*} b, \\
& \left\langle\sigma_{y}\right\rangle=1\left(a b^{*}-a^{*} b\right), \\
& \left\langle\sigma_{z}\right\rangle=a^{*} a-b^{*} b,
\end{aligned}
$$

and thus

$$
\left\langle\sigma_{x}\right\rangle^{2}+\left\langle\sigma_{y}\right\rangle^{2}+\left\langle\sigma_{z}\right\rangle^{2}=\left(a^{*} a+b{ }^{*} b\right)^{2}
$$

Therefore

$$
|\overrightarrow{\mathrm{P}}|=1 \text {. }
$$

Hence in this case $\vec{P}$ is a unit vector whose components are the expectation values of the components of $\vec{\sigma}$. The spinor $|x\rangle$ contains the 
information about the direction of the spin. The direction is defined as that given by the polarization vector of eq. (1.28).

For a spin $1 / 2$ particle in a state $|\chi\rangle$ there always exists a direction of quantization $\hat{u}$ such that the particle has spin projection $+1 / 2$ along it, that is

$$
\hat{u} \cdot \text { む }|x\rangle=|x\rangle \text {. }
$$

To show that the direction $\hat{u}$ is given by $\vec{P}$, we take the $|x\rangle$ expectation value of the identity

$$
\sigma_{1} \sigma_{j}+\sigma_{j} \sigma_{1}=2 \delta_{i j}, 1, j=1,2,3
$$

and find

$$
\left\langle x\left|\sigma_{i} \sigma_{j}\right| x\right\rangle+\left\langle x\left|\sigma_{j} \sigma_{i}\right| x\right\rangle=2 \delta_{i j}
$$

Multiplying this relation by $u_{i}$ and sumining over $i$, we find

$$
\left\langle x\left|\hat{u} \cdot \vec{\sigma} \sigma_{j}\right| x\right\rangle+\left\langle x\left|\sigma_{j} \hat{u} \cdot \vec{\sigma}\right| x\right\rangle=2 u_{j},
$$

and after using eq. (1.32)

$$
2\left\langle x\left|\sigma_{j}\right| x\right\rangle=2 u_{j} .
$$

Thus

$$
\hat{\mathbf{u}}=\langle\overrightarrow{\mathrm{J}}\rangle=\overrightarrow{\mathrm{P}}
$$

We have already shown that the degree of polarization of the spinor $|x\rangle$ given by eq. (1.30) is one, the spinor $|\chi\rangle$ hence describes a fully polarized state, regardless of the cholce of $a$ and $b$. The components $a$ and $b$ only determine the direction of $\stackrel{+}{\mathrm{P}}$, but not its magnitude. The result is due to the fact that a wave function describes a fure state. The spinor $|\chi\rangle$ describes a coherent superposition of the two states with spin up and spin down. A partially polarized or an unpolarized 
beam or target must be described as an incoherent superposition of the two spin states, a so-called mixed state.

A mixed state is described by a density matrix, rather than by a state vector. Apart from the spin orientation we assume that all the other quantum numbers of the particles forming the target are the same and so we need only introduce the spin density matrix.

Incoherent superposition means, by definition, that to calculate the probability of finding a certain experimental result with a system in a mixed state one must first calculate the probability for each of the pure states and then take an average, attributing to each of the pure states an assigned "weight". However, the description of a nonpure state as the incoherent superposition of pure states is not unique. In an unpolarized beam (or target) of spin $1 / 2$ particles, the spin directions of the particles in the bean are randomly distributed. An unpolarized beam $(|\vec{P}|=0)$ may be considered as an equal mixture of any two completely and oppositely polarized beams.

The density operator $\rho$ is defined as 19

$$
\rho=\sum_{\alpha} \mid \alpha>g_{\alpha}\langle\alpha| \text {, }
$$

where the sum runs over a complete set of normalized states $|\alpha\rangle$ with weights $g_{\alpha}$, and

$$
\langle\alpha \mid \alpha\rangle=1, \quad \sum_{\alpha} g_{\alpha}=1 .
$$

The average of an observable $Q$ for the ensemble is

$$
\overline{\langle Q\rangle}=\operatorname{tr}(\rho Q) \text {, }
$$


where the expectation value for a pure state is denoted by \langle\rangle ; the ensemble average by a bar, and "tr" denotes the trace. Thus the knowledge of $\rho$ enables us to compute the statistical average of $Q$. From eq. (1.33) $\rho$ is Herintian and the trace of $\rho$ is unity

$$
\rho t=\rho, \operatorname{tr}(\rho)=1 \text {. }
$$

An unpolarized beam $(\vec{P}=0)$ of spin $1 / 2$ particles may be considered as an equal mixture of two completely and oppositely polarized beams. For example the incoherent mixture of spin up $|+\rangle=\left(\frac{1}{0}\right)$, and spin down $|-\rangle=\left(\begin{array}{l}0 \\ 1\end{array}\right)$ states with equal weights, $g_{+}=g_{-}=1 / 2$. According to eq. (1.33) the density operator is

$$
\begin{aligned}
\rho & =\sum_{\alpha}|\alpha\rangle g_{\alpha}\langle\alpha| \\
& =g_{+}|+\rangle\left\langle+\left|+g_{-}\right|-\right\rangle\langle-| \\
& =\frac{1}{2}\left(\begin{array}{l}
1 \\
0
\end{array}\right)\left(\begin{array}{ll}
1 & 0
\end{array}\right)+\frac{1}{2}\left(\begin{array}{l}
0 \\
1
\end{array}\right)\left(\begin{array}{ll}
0 & 1
\end{array}\right) \\
& =\frac{1}{2}\left(\begin{array}{ll}
1 & 0 \\
0 & 1
\end{array}\right)
\end{aligned}
$$

or

$$
p=\frac{1}{2} \mathrm{~T}
$$

The polarization vector is

$$
\vec{P}=\overrightarrow{\langle\vec{\sigma}\rangle}=\operatorname{tr}(\rho \vec{\sigma})=\frac{1}{2} \operatorname{tr}(\vec{\sigma})=0
$$

The incoherent mixture of the two states $|+\rangle$ and $|\rightarrow\rangle$ with equal weights indeed represents an unpolarized bean.

The differential cross section $1 \mathrm{~s} 20$

$$
\begin{aligned}
\frac{d \sigma}{d n} & =\left\langle\bar{M} T_{M}\right. \\
& =\operatorname{tr}\left(M{ }^{\prime} \rho_{i}\right) .
\end{aligned}
$$


Using eq. (1.6) for $M$ and $\rho_{i}=(1 / 2) I$ for unpolarized iniclal state we find

$$
\begin{aligned}
\left(\frac{d \sigma}{d \Omega}\right)_{\text {unpol }} & =\frac{1}{2} \operatorname{tr}(M M \dagger) \\
& =\frac{1}{2} \operatorname{tr}\left[(f+g \vec{\sigma} \cdot \hat{n})\left(f^{*}+g^{*} \vec{\sigma} \cdot \hat{n}\right)\right] \\
& =|f|^{2}+|g|^{2},
\end{aligned}
$$

in agreement with $(1.20)$.

The density matrix, $\rho_{f}$, for the scattered beam particles from an incident beam described by the density matrix $\rho_{i}$ :

$$
\rho_{i}=\sum_{i} g_{i}\left|x_{i}\right\rangle\left\langle x_{i}\right|
$$

can be found with the help of the scattering matrix M. The amplitude of the scattered spin state $\left|x_{f}\right\rangle$ arising from a pure initial state $\left|x_{i}\right\rangle$ is $N M\left|X_{1}\right\rangle$, where $N$ is a normalization factor. From eq. (1.33) the density matrix for the scattered beam is

$$
\rho_{f}=\sum_{i} g_{i}\left|x_{f}\right\rangle\left\langle x_{f}\right|
$$

Substituting $\left|x_{\mathrm{f}}\right\rangle=\mathrm{NM}\left|x_{1}\right\rangle$ we find

$$
\begin{aligned}
\rho_{f} & =\sum_{i} g_{i}|N|^{2}\left|M x_{i}\right\rangle\left\langle M x_{i}\right| \\
& =|N|^{2} M\left(\sum_{i} g_{i}\left|x_{i}\right\rangle\left\langle x_{i}\right|\right) M^{\dagger} \\
& =|N|^{2} M \rho_{i} M^{\dagger} .
\end{aligned}
$$

The condition $\operatorname{tr}\left(\rho_{\mathrm{f}}\right)=1$ determines $|N|^{2}$ and $\rho_{\mathrm{f}}$ becomes

$$
\rho_{f}=\frac{M \rho_{i} M^{\dagger}}{\operatorname{tr}\left(M \rho_{i} M^{\dagger}\right)} \text {. }
$$

We may use the density matrix formalism to obtain the polarization $\overrightarrow{\mathrm{P}}_{f}$ of che scattered beam. Writing

$$
\overrightarrow{\mathrm{P}}_{\mathrm{f}}=\overline{\langle\vec{\sigma}\rangle}=\operatorname{tr}\left(\rho_{\mathrm{f}} \vec{\sigma}\right),
$$


and using eq. (1.38), we have

$$
\overrightarrow{\mathrm{P}}_{\mathrm{f}}=\frac{\operatorname{tr}\left(M \rho_{i} \mathrm{M}^{\dagger} \mathrm{z}\right)}{\operatorname{tr}\left(M \rho_{i} M^{\dagger}\right)} .
$$

For example, if the system is initially unpolarized, so that $\mid \vec{P}_{1}^{\prime}=0$, we have $\rho_{1}=(1 / 2) I$ and

$$
\begin{aligned}
\operatorname{tr}\left(M \rho_{i} M^{\dagger}\right) & =\frac{1}{2} \operatorname{tr}\left[(f+g \xi \cdot \hat{n})\left(\tilde{I}^{\star}+g^{\star} \text { を・ } \hat{n}\right) \xi\right] \\
& =\left(f g^{\star}+f^{\star} g\right) \hat{n} .
\end{aligned}
$$

We also have in this case

$$
\operatorname{tr}\left(M \rho_{i} M^{\dagger}\right)=\frac{1}{2} \operatorname{tr}\left(M M^{\dagger}\right)=|f|^{2}+|g|^{2},
$$

therefore

$$
\overrightarrow{\mathrm{P}}_{\mathrm{f}}=\frac{\mathrm{fg}^{*}+\mathrm{f}^{*} \mathrm{~g}}{|\mathrm{f}|^{2}+|\mathrm{g}|^{2}} \hat{\mathrm{n}},
$$

in agreement with our previous result eq. $(1.26)$. The polarization $\overrightarrow{\mathrm{P}}_{\mathrm{f}}$ becomes zero when either $f(\theta)$ or $g(\theta)$ vanishes (or $f$ and $g$ are $90^{\circ}$ out of phase). Since the central force does not glve rise to a spin-flip scattering (that is $g=0$ ), $\overrightarrow{\mathrm{P}}_{\mathrm{f}}$ is necessarily zero if the scattering is caused by a central force only (e.g. in pure Coulomb scattering).

Let us now find the density operator for a beatn (or target) with polarization $\vec{P}$, with $0 \leqslant|\vec{P}| \leqslant 1$. Consider an ensemble of spin $1 / 2$ particles. In this case the spin orientation is represented by a $2 \times 2$ density matrix which can depend at most on three real parameters. Because the unit $2 \times 2$ matrix $I$ and the three Paull spin matrices form a complete set of $2 \times 2$ matrices, we may write the density matrix as:

$$
\rho=A_{0} I+A_{x} \sigma x+A_{y} \sigma y+A_{z} \sigma_{z}
$$


where $A_{0}, A_{x}, A_{y}, A_{z}$ are four complex parameters. We may use a slightly different notation and write this as

$$
\rho=A_{0} I+\vec{A} \cdot \vec{\sigma}
$$

Since $\operatorname{tr}(\rho)=1, \operatorname{tr}(I)=2$, and $\operatorname{tr}\left(\sigma_{i}\right)=0$, we see that

$$
\mathrm{A}_{\mathrm{O}}=\frac{1}{2}
$$

The mean polarization is

$$
\vec{P}=\overrightarrow{\langle\vec{\sigma}\rangle}=\operatorname{tr}(\vec{\rho})
$$

Substitution of eq. (1.40) into this expression yields

$$
\overrightarrow{\mathrm{P}}=2 \overrightarrow{\mathrm{A}} \text {. }
$$

Summarizing these results, we may write $(1.40)$ as

$$
\rho=\frac{1}{2}(1+\vec{P}, \vec{\sigma}) \text {. }
$$

The differential cross section for scattering from an initial state with polarization $\overrightarrow{\mathrm{P}}_{i}$ and density matrix $\rho_{i}$ is given by $(1.37)$ :

$$
\frac{\mathrm{d} \sigma}{\mathrm{d} \Omega}=\operatorname{tr}\left(M \rho_{i} M^{\dagger}\right)
$$

Substitution Jf $(1.6)$ and $(1.43)$ yields

$$
\begin{aligned}
\frac{d \sigma}{d \Omega} & =\operatorname{tr}\left[(f+g \vec{\sigma} \cdot \hat{n}) \frac{1}{2}\left(1+\vec{P}_{i} \cdot+\vec{\sigma}\right)\left(f^{*}+g^{*} \vec{\sigma} \cdot \hat{n}\right)\right] \\
& =|f|^{2}+|g|^{2}+2 \operatorname{Re}\left(f g^{*}\right) \vec{P}_{i} \cdot \hat{n} .
\end{aligned}
$$

We note that this result has exactly the same form as eq. (1.19), but it is more general since we may now have $0 \leqslant\left|\vec{P}_{1}\right| \leqslant 1$.

$$
\begin{aligned}
& \text { From eq. (1.39) the polarization } \vec{P}_{f} \text { of the scattered beam is } \\
& \overrightarrow{\mathrm{P}}_{\mathrm{f}}=\frac{\operatorname{tr}\left(M \rho_{i} M{ }^{\prime} t\right)}{\operatorname{tr}\left(M \rho_{1} M^{\dagger}\right)} \\
& =\frac{\left(|f|^{2}-|g|^{2}\right) \vec{P}_{i}+\left(f g^{*}+f^{*} g+2|g|^{2 *} \vec{P}_{i} \cdot \hat{n}\right) \hat{n}+i\left(f g^{*}-f^{*} g\right) \vec{P}_{i} \times \hat{n}}{|f|^{2}+|g|^{2}+\left(f g^{*}+f^{*} g\right) \vec{P}_{i} \cdot \hat{n}} .
\end{aligned}
$$


Again this result has the same form as eq. (1.25), but is more general, since now $\left|\vec{P}_{i}\right|$ may have any value in the range $0 \leqslant\left|\vec{P}_{i}\right|<1$.

We may write the differential cross section eq. (1.44) for scattering off a transversely polarized target in terms of the unpolarized differential cross section (1.26) as

or by defining

$$
\frac{\mathrm{d} \sigma}{\mathrm{d} \Omega}=\left(\frac{\mathrm{d} \sigma}{\mathrm{d} \Omega}\right)_{\text {unpol }}\left(1+\frac{\mathrm{fg}^{\star}+\mathrm{f}^{*} \mathrm{~g}}{|\mathrm{f}|^{2}+|\mathrm{g}|^{2}} \overrightarrow{\mathrm{P}}_{\mathrm{i}} \cdot \hat{\mathrm{n}}\right),
$$

we find

$$
A_{N}(\theta)=\frac{f^{*}+f^{*} g}{|f|^{2}+|g|^{2}}=\frac{2 \operatorname{Re}\left(f^{*}\right)}{|f|^{2}+|g|^{2}},
$$

$$
\frac{d \sigma}{d \Omega}=\left(\frac{d \sigma}{d \Omega}\right)_{\text {unpol }}\left(1+A_{N}(\theta) \vec{P}_{i}{ }^{2} \hat{n}\right) \text {. }
$$

The second term within the parenthesis gives the effect of a polarized incident beam. If we use the symbol $\phi_{0}$ to denote the angle between $\overrightarrow{\mathrm{P}}_{\boldsymbol{i}}$ and $\hat{n}$; that is $\vec{P}_{i} \cdot \hat{n}=P_{i} \cos \phi_{0}$ and denote $\frac{d \sigma}{d \Omega}$ by $I\left(\theta, \phi_{0}\right)$ and $\left(\frac{d \sigma}{d \Omega}\right)_{\text {unpol }}$ by $I_{0}(\theta)$ then we have

$$
I\left(\theta, \phi_{0}\right)=I_{0}(\theta)\left(1+A_{N}(\theta) \vec{P}_{i} \cdot \hat{n}\right),
$$

where $A_{N}(\theta)$ is the "analyzing power" of the reaction.

Polarization experiments play a vital role in the partial wave (or phase shift) analysis of scattering processes involving particles with spin. In the study of pion-nucleon scattering the measurements of the unpolarized differential cross section $I_{0}(\theta)$ is not sufficient to determine the partial wave amplitudes unambiguously. However if these itta are supplemented by measurements of the analyzing power $A_{N}(\theta)$ (together with spin rotation parameters), then these ambiguities can be 
resolved, and in particular the spin and parities of the resonant states formed by the pion-nucleon system can be predicted.

It is therefore relevant to ask how $A_{N}(\theta)$ can be measured. Suppose that the initial polarization $\stackrel{\vec{P}}{\mathbf{i}}_{\text {, of }}$ the proton target is directed upwards (in other words we take the direction of $\vec{P}_{i}$ co define the "up" direction) and that it is normal to the plane of scattering. Here we adopt the viewpoint of an observer upright in the c.m. frame looking in the direction of incident proton beam. From our viewpoint î is vertically upwards for scattering to the left and verically downwards for scattering to the right.* We denote the cross section for scattering to the left $\left(p_{0}=0, \ddot{\mathrm{r} i}\right.$ parallel to $\left.\overrightarrow{\mathrm{P}}_{\mathbf{i}}\right)$ by $\mathrm{L}$ and for scattering to the right $\left(\phi_{0}=\pi, \hat{\mathrm{n}}\right.$ antiparallel to $\left.\overrightarrow{\mathrm{P}}_{\hat{i}}\right)$ by $\mathrm{R}$. It therefore follows from eq. (1.48) that the intensities for left and right scattering are in the ratio

$$
\frac{L}{R}=\frac{1+A_{N}(\theta) P_{i}}{1-A_{N}(\theta) P_{i}},
$$

where $P_{i} \equiv\left|\vec{P}_{i}\right|$, and so we have

$$
P_{i} A_{N}(\theta)=\frac{L-R}{L+R} \equiv \varepsilon
$$

$E$ is called the left-right asymmetry.

*Or one can say that particles are scattered to the left if $\hat{\mathbf{n}}$ is parallel to $\overrightarrow{\mathrm{P}}_{i}$ and to the right if $\hat{\mathrm{n}}$ is antiparallel to $\overrightarrow{\mathrm{P}}_{\mathcal{I}}$, in
agreement with the Basel convention. 


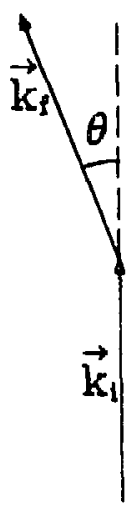

(a)

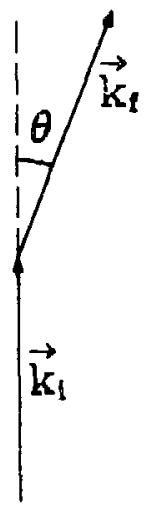

(b)

Figure 1.3 (a) Scattering to the left. (b) Scattering to the right.

The analyzing power $A_{N}(\theta)$ is therefore obtained by a measurement of the left-right asymetry in the scattering. This asymetry occurs even though we have parity conservation.

The actual number of counts recorded in detectors are

$$
L=n N \Omega E I_{0}\left(1+A_{N}(\theta) P_{1}\right)
$$

and when we flip the polarization, i.e. $\vec{P}_{i} \rightarrow-\vec{P}_{i}$, we obtain

$$
L^{-}=n^{-} N \cap E^{-} I_{0}\left(1-A_{N}(\theta) P_{i}\right) \text {, }
$$

where $n\left(n^{\prime}\right)$ is the number of incident particles, $N$ is the target thickness (atoms $/ \mathrm{cm}^{2}$ ), $\lambda$ is the solid angle, and $E\left(E^{\prime}\right)$ is the efficiency. From rotational and parity invariance, the probability of scattering to the left through $\theta$ with target polarization down is the same as scattering to the right through $\theta$ wilen polarization is up $\left(L^{\prime}=R\right)$. 


\subsection{Spin Rotation Parameters}

We have seen that the scattering amplitude $M$ involves two independent complex functions of energy and scattering angle for spin zero - spin one-half scattering. Clearly a simple scattering experiment to measure the differential cross section as a function of energy and scattering angle can not provide more than one relation between these functions. It is for this reason that experiments are performed with polarized targets, since polarization data give further relations among the parameters of the scattering amplitude.

Suppose we have measured the pion-nucleon unpolarized differential cross section $I_{0}$, and the analyzing power $A_{N}(\theta)$ through the measurement of left-right asymetry using a target polarized transversely to the scattering plane. In terms of scattering amplitudes $f$ and $g$ we have

$$
\begin{aligned}
& I_{0}=|f|^{2}+|g|^{2}, \\
& I_{O} A_{N}=f g^{*}+f^{*} g .
\end{aligned}
$$

Adding and subtracting these two equations we find

$$
\begin{aligned}
& |f+g|^{2}=I_{0}\left(1+A_{N}\right), \\
& |f-g|^{2}=I_{0}\left(1-A_{N}\right) .
\end{aligned}
$$

Thus, $I_{0}$ and $A_{N}$ dete ine the magnitude of $f+g$ and $f-g$. We may write

$$
\begin{aligned}
& \mathrm{f}+\mathrm{g}=\sqrt{\mathrm{I}_{\mathrm{O}}\left(1+\mathrm{A}_{\mathrm{N}}\right)} \mathrm{e}^{i(\alpha-\beta / 2)} \\
& \mathrm{f}-\mathrm{g}=\sqrt{\mathrm{I}_{\mathrm{C}}\left(1-\mathrm{A}_{\mathrm{N}}\right)} \mathrm{e}^{i(\alpha+\beta / 2)}
\end{aligned}
$$


defining the phase factors $\alpha$ and $\beta$. From these two equations we Eind

and hence

$$
\begin{aligned}
\sin \beta & =\frac{2 \operatorname{Im}\left(f_{g}^{*}\right)}{I_{0} \sqrt{1-A_{N}^{2}}} \\
\cos \beta= & \frac{|f|^{2}-|g|^{2}}{I_{0} \sqrt{1-A_{N}^{2}}}
\end{aligned}
$$

$$
\tan B=\frac{2 \operatorname{Im}\left(\mathrm{fg}^{*}\right)}{|f|^{2}-|g|^{2}}
$$

Let us now discuss the pilarization of the recoil proton $\overrightarrow{\mathrm{P}}_{\mathrm{f}}$ when the initial polarization $\vec{P}_{1}$ lies in the scattering plane and is perpendicular to the incident momentum $\vec{k}_{i}$. We will use the projectile helicity frame for describing the initial polarization and the outgoing laboratory helicity frame for the recoil proton. Then $\overrightarrow{\mathrm{P}}_{i}=\mathrm{P}_{i} \hat{\mathrm{u}}_{\mathrm{x}}$ where $\hat{\mathbf{u}}_{\mathrm{x}}, \hat{\mathrm{u}}_{\mathrm{y}}, \hat{\mathbf{u}}_{\mathrm{z}}$ are unit vectors $\mathrm{a}_{i} \mathrm{j}$, the $\mathrm{x}, \mathrm{y}$, and $z$-axes of the projectile helicity frame and similarly $\hat{\mathrm{u}}_{\mathrm{x}^{-}}, \hat{\mathrm{u}}_{\mathrm{y}^{-}}$, and $\hat{\mathrm{u}}_{\mathrm{z}^{-}}$are unit vectors along the $x^{\prime}, y^{\prime}$, and $z^{\prime}$-axes of the outgoing proton laboratory helicity frame. From $(1.44)$ it is seen that

$$
\frac{\mathrm{d} \sigma}{\mathrm{d} \Omega}=\mathrm{I}_{0}
$$

From eq. (1.45) the recoil proton's polarization is

$$
I_{0} \vec{P}_{f}=\left(|f|^{2}-|g|^{2}\right) P_{i} \hat{u}_{x}+\left(f g^{*}+f^{*} g\right) \hat{u}_{y}+i\left(f g^{*}-f^{*}{ }_{g}\right) P_{i} \hat{u}_{x} \times \hat{u}_{y}
$$

The recoil proton laboratory helicity frame is obtained from the projectile helicity frame by a rotation around the $y-\left(\right.$ or $y^{-}-$) axis by the recoll proton laboratory scattering angle $\theta_{L}$, and therefore

$$
\begin{aligned}
& \hat{\mathrm{u}}_{\mathrm{x}}=\cos \theta_{\mathrm{L}} \hat{\mathrm{u}}_{\mathrm{x}^{-}}+\sin \theta_{\mathrm{L}} \hat{\mathrm{u}}_{\mathrm{z}^{-}}, \\
& \hat{\mathrm{u}}_{\mathrm{y}}=\hat{\mathrm{u}}_{\mathrm{y}^{-}}, \\
& \hat{\mathrm{u}}_{\mathrm{z}}=-\sin \theta_{\mathrm{L}} \hat{\mathrm{u}}_{\mathrm{x}^{-}}+\cos _{\mathrm{L}} \hat{\mathrm{u}}_{\mathrm{z}^{-}}
\end{aligned}
$$




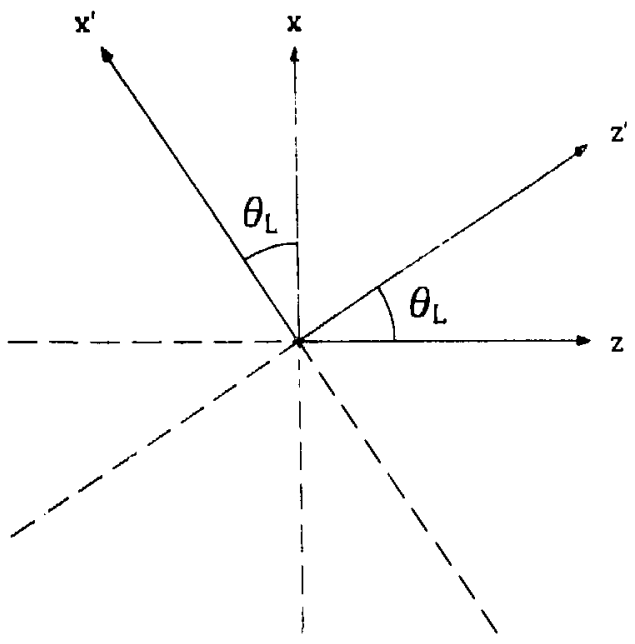

Figure 1.4. Projectlle hellcity frame and recoll proton laboratory helicity frame.

Hence, $\overrightarrow{\mathrm{P}}_{\mathrm{f}}$ in the recoil proton laboratory helicity frame is

$$
\begin{aligned}
\mathrm{I}_{\mathrm{O}} \overrightarrow{\mathrm{P}}_{\mathrm{f}}= & \mathrm{P}_{\mathrm{I}}\left[\left(|\mathrm{f}|^{2}-|\mathrm{g}|^{2}\right) \cos \theta_{\mathrm{L}}-\mathrm{i}\left(\mathrm{fg}^{\star}-\mathrm{f}^{\star} \mathrm{g}\right) \sin \theta_{\mathrm{L}}\right] \hat{\mathrm{u}}_{\mathrm{x}^{-}} \\
& +\left(\mathrm{fg}{ }^{\star}+\mathrm{f}^{\star} \mathrm{g}\right) \hat{\mathrm{u}}_{\mathrm{y}^{-}} \\
& +\mathrm{P}_{1}\left[\left(|\mathrm{f}|^{2}-|\mathrm{g}|^{2}\right) \sin \theta_{\mathrm{L}}+\mathrm{i}\left(\mathrm{fg}{ }^{\star}-\mathrm{f}^{\star} \mathrm{g}\right) \cos \theta_{\mathrm{L}}\right] \hat{\mathrm{u}}_{\mathrm{z}^{-}} \cdot
\end{aligned}
$$

Using the definition of analyzing power $A_{N}$ and defining the $A$ and $R$ parameters by*

$$
\begin{aligned}
& I_{0} R=\left(|f|^{2}-|g|^{2}\right) \cos \theta_{L}+2 \operatorname{Im}\left(f g^{*}\right) \sin \theta_{L}, \\
& I_{0} A=\left(|f|^{2}-|g|^{2}\right) \sin \theta_{L}-2 \operatorname{Im}\left(f g^{*}\right) \cos \theta_{L},
\end{aligned}
$$

the polarization becomes

$$
\overrightarrow{\mathrm{P}}_{\mathrm{F}}=R \mathrm{P}_{\mathrm{i}} \hat{\mathrm{u}}_{\mathrm{x}^{\prime}}+\mathrm{A}_{\mathrm{N}^{\prime}} \hat{\mathrm{u}}_{\mathrm{y}^{-}}+\mathrm{AP}_{\mathrm{i}} \hat{\mathrm{u}}_{\mathrm{z}^{\prime}} .
$$

From eqs. ( 1.58$)$ and $(1.59)$ it follows that

$$
\begin{aligned}
|f|^{2}-|g|^{2} & =I_{0} \sqrt{1-A_{N}^{2}} \cos \beta, \\
2 \operatorname{Im}\left(E g^{*}\right) & =I_{D} \sqrt{1-\dot{H}_{N}^{2}} \sin \beta .
\end{aligned}
$$

${ }_{\mathrm{A}}$ and $\mathrm{R}$ were named $\mathrm{D}_{\ell S}$ and $\mathrm{D}_{\mathrm{Ss}}$ respectively in the Ann Arbor
convention. 
Substituting these into $(1.61)$ we find

$$
\begin{aligned}
& R=\sqrt{1-A_{N}^{2}} \cos \left(\beta-\theta_{L}\right), \\
& A=\sqrt{1-A_{N}^{2}} \sin \left(\theta_{L}-\beta\right) .
\end{aligned}
$$

Thus the recoil polarization is

$$
\overrightarrow{\mathrm{P}}_{\mathrm{f}}=\mathrm{A}_{\mathrm{N}} \hat{\mathrm{u}}_{\mathrm{y}^{-}}+\mathrm{P}_{\mathrm{i}} \sqrt{\mathrm{l}-\mathrm{A}_{N^{2}}^{2}}\left[\cos \left(\beta-\theta_{\mathrm{L}}\right) \hat{\mathrm{u}}_{\mathrm{x}^{-}}+\sin \left(\theta_{\mathrm{L}^{-\beta}}\right) \hat{\mathrm{u}}_{\mathrm{z}^{-}}\right]
$$

The recoil polarization is completely deterinined by the analyzing power $A_{-N}$ and $B$ at each scattering angle ${ }_{L}$.

If the initial polarization $\overrightarrow{\mathrm{P}}_{i}$ is in the direction of Incident beam i.e. $\vec{P}_{1}=P_{i} \hat{u}_{z}$, then again from eq. (1.44) the differential cross section is

$$
\frac{\mathrm{d} \sigma}{\mathrm{d} \Omega}=\mathrm{I}_{0}
$$

and the recoil proton polarization is

$$
\overrightarrow{\mathrm{P}}_{f}=\mathrm{K}^{\prime} \mathrm{P}_{i} \hat{\mathrm{u}}_{\mathrm{x}^{-}}+\mathrm{A}_{\mathrm{N}} \hat{\mathrm{u}}_{\mathrm{y}^{\prime}}+\mathrm{A}^{-} \mathrm{P}_{i^{-}} \hat{\mathrm{u}}_{z^{-}}
$$

where

$$
\begin{aligned}
& R^{\prime}=\sqrt{1-A_{N}^{2}} \sin \left(\beta-\theta_{L}\right), \\
& A^{\prime}=\sqrt{1-A_{N}^{2}} \cos \left(B-\theta_{L}\right) .
\end{aligned}
$$

Comparing eqs. $(1.63)$ and $(1.66)$ we find

$$
\begin{aligned}
& A^{-}=R, \\
& R^{-}=-A
\end{aligned}
$$

To find out about the physical meaning of $\beta$, the spherirn1 coordinates $\theta, \phi$ that define $\hat{P}_{f}$ may be obtained as follows. If the 
initial spin direction $\hat{\mathrm{P}}_{i}$ is defined by spherical coordinates $\theta_{i}, \phi_{i}$ and the $z$-axis is chosen along $\hat{n}$, then

$$
\left|\chi_{f}\right\rangle=M\left|x_{i}\right\rangle=\left(\begin{array}{c}
f+g 0 \\
0 f-g
\end{array}\right)\left(\begin{array}{l}
\cos \left(\theta_{i} / 2\right) e^{-i \phi_{1} / 2} \\
\sin \left(\theta_{i} / 2\right) e^{+i \phi_{i} / 2}
\end{array}\right) \cdot
$$

Eigenstates of $\vec{\jmath} \cdot \hat{n}$ scatter independently, but with a definite relative phase which induces spir rotation effects. Substituting eqs. (1.56) and $(1.57)$ into $(1.68)$

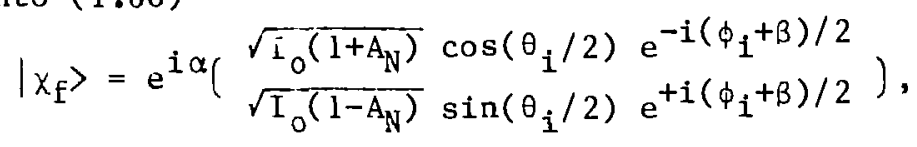

and comparing the result with

$$
\left|x_{f}\right\rangle=\left(\begin{array}{ll}
\cos (\theta / 2) & e^{-i \phi / 2} \\
\sin (\theta / 2) & e^{+i \phi / 2}
\end{array}\right)
$$

we find (except for the overall phase $\alpha$ )

$$
\begin{gathered}
\phi=\phi_{i}+\beta, \\
\sqrt{\mathrm{I}_{0}\left(1+A_{N}\right)} \cos \left(\theta_{i} / 2\right)=\cos (\theta / 2), \\
\sqrt{I_{0}\left(1-A_{N}\right)} \sin \left(\theta_{i} / 2\right)=\sin (\theta / 2) .
\end{gathered}
$$

Squaring the last two equations, and then subtracting and adding the results we find

$$
\begin{aligned}
& I_{0}\left(\cos \theta_{i}+A_{N}\right)=\cos \theta, \\
& I_{0}\left(1+A_{N} \cos \theta_{i}\right)=1,
\end{aligned}
$$

and therefore

$$
\cos \theta=\frac{\cos \theta_{i}+A_{N}}{1+A_{N} \cos \theta_{i}}
$$

In words, the spin of the partic ${ }^{1}$ e is bent toward the normal to the scattering plane $\hat{n}$ by an amount determined by the analyzing power $A_{N}$ and the original z-component of spin, whereas the direction of the projection of the spin in the scattering plane is rotated through the angle $\beta$. 
In order to determine $\beta$ we must measure the rotation of the projection of the spin vector in the scattering plane. Such an experiment is illustrated in figure 1.5. The initial direction of polarization is chosen to lie in the scattering plane. Since a simple analyzer can only detect the components of the final polarization vector in the plane perpendicular to the outgolng direction $\vec{k}_{f}$, the analyzer is chosen so as to detect the component of polarization along $\hat{u}_{\mathrm{x}}$.. This component is called $\mathrm{R}$ and if the incident beam is completely polarized is given by

$$
R=\sin \theta \cos \left(90^{\circ}-\left(\phi-\theta_{L}\right)\right)
$$

Using eq. ( 1.69$)$ with $\theta_{1}=90^{\circ}, \phi_{i}=90^{\circ}$ we find

$$
\begin{aligned}
& \cos \theta=A_{N}, \\
& \phi=90^{\circ}+B,
\end{aligned}
$$

and $\mathrm{R}$ becomes

$$
R=\sqrt{1-A_{N}^{2}} \cos \left(B-\theta_{L}\right)
$$

The component of $\overrightarrow{\mathrm{P}}_{\mathrm{f}}$ along $\overrightarrow{\mathrm{k}}_{\mathrm{f}}$ is

$$
A=\sin \theta \cos \left(\phi-\theta_{L}\right)=\sqrt{1-A_{N}^{2}} \sin \left(\theta_{L}-\beta\right),
$$

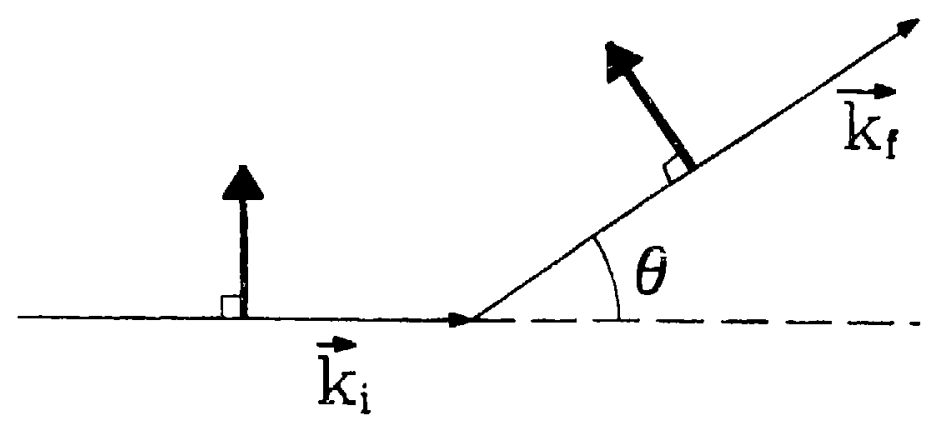

Figure 1.5. The direction of polarization is indicated by an arrow on the beam. The arrow on the outgoing beam indicates the normal to the second scattering plane and thus the component of polarization to be measured. 
and its component along the normal to the scattering plane is

$$
\cos \theta=A_{N}
$$

Physically, $B$ is the angle in the scattering plane through which the proton polarization is rotated by the $\pi-p$ interaction. If both $A$ and $R$ are measured, the angle $\beta$ can be obtained directly from their ratio. Using eqs. (1.63) we find

$$
\frac{A}{R}=\tan \left(\theta_{L}-\beta\right)
$$

where $\theta_{L}$ is the recoil proton laboratory scattering angle, i.e. the angle between the incident pion and the recoll proton in the laboratory frame. A knowledge of $R$ alone leaves the sign of $\beta$ undetermined. Using eq. (1.63) it is seen that

$$
A^{2}+R^{2}+A_{N}^{2}=1
$$

To determine $A(R)$ it is necessary to align the target polarization along $\hat{u}_{z}$ (or $\hat{u}_{x}$ ) and to measure the recoil proton scattering asymmetry in a plane perpendicular to the plane of the initial scattering. The up-down asymmetry in the second scattering is

$$
\varepsilon_{R}=\frac{I(\text { down })-I(\text { up })}{I(\text { down })+I(\text { up })}=P_{c} R P_{i},
$$

if the polarization of the target is directed along $\hat{u}_{x}$, and

$$
\varepsilon_{A}=\frac{I^{-}(\text {down })-I^{-}(\text {up })}{I^{\prime}(\text { down })+I^{\prime}(\text { up })}=P_{c^{A}} P_{i} \text {, }
$$

if the target polarization is $\vec{P}_{i}=P_{i} \hat{u}_{z}$. Here $P_{c}$ is the analyzing power of the analyzer target, and the "up" direction is deternined by the direction of the vector $\hat{u}_{z} \times \hat{u}_{z^{-}}$. Notice that while $A_{N}$ is invariant in 
going from laboratory to center of mass frame, other spin parameters are not invariant under this boost.

We summarize the observed quantities

$$
\begin{aligned}
& I_{0}=|f|^{2}+|g|^{2}, \\
& I_{0} A_{N}=2 \operatorname{Re}\left(f g^{*}\right), \\
& I_{O} R=\left(|f|^{2}-|g|^{2}\right) \cos \theta_{L}+2 \operatorname{Im}\left(f g^{*}\right) \sin \theta_{L}, \\
& I_{0} A=\left(|f|^{2}-|g|^{2}\right) \sin \theta_{L}-2 \operatorname{Im}\left(f g^{*}\right) \cos \theta_{L} .
\end{aligned}
$$

From eqs. $(1.75 c)$ and $(1.75 d)$ it follows that

$$
\begin{aligned}
|f|^{2}-|g|^{2} & =I_{0}\left(R \cos \theta_{L}+A \sin \theta_{L}\right), \\
2 \operatorname{Im}\left(f g^{*}\right) & =I_{0}\left(R \sin \theta_{L}-A \cos \theta_{L}\right) .
\end{aligned}
$$

From eqs. $(1.75 a),(1.75 b),(1.76 a)$, and $(1.76 b)$ we find

$$
\begin{aligned}
& |f|^{2}=\frac{1}{2} I_{O}\left(1+R \cos \theta_{L}+A \sin \theta_{L}\right), \\
& |g|^{2}=\frac{1}{2} I_{O}\left(1-R \cos \theta_{L}-A \sin \theta_{L}\right), \\
& f g^{*}=\frac{1}{2} I_{O}\left(A_{N}+i R \sin \theta_{L}-i A \cos \theta_{L}\right) .
\end{aligned}
$$

Let us now discuss a complete set of measurements that determine the scattering matrix $M$. of the four real quantities in $M$ ( $t$ he real and imaginary parts of $f$ and $g$ ) it is possible to find three by straightforward experiments.

First, the unpolarized differential cross section is determined in a scattering of pions off an unpolarized liquid hydrogen target. 
Second, the analyzing power is determined by the measurement of left-right asymmetry using a transversely polarized target.

Third, $B$ is given by the $A$ and $R$ parameters, and requires the measurement of a transverse polarization produced in scattering from a polarized target.

$\beta$ can only be measured by detecting the recoil polarization from a target polarized with a component in the scattering plane; in particular $\beta$ is independent of $I_{0}$ and $A_{N}$.

While a measurement of the analyzing power determines $\operatorname{Re}\left(\mathrm{fg}^{*}\right)$ a measurement of $A$ and $R$ determines $\operatorname{Im}\left(\mathrm{fg}^{*}\right)$. These three experiments will give the moduli of $f$ and $g$ and the phase angle between them. In other words at any scattering angle $\theta$ the amplitudes $f$ and $g$ are determined except for the common phase $\alpha$. In the region where the nuclear and Coulomb amplitudes are comparable this phase factor can be determined by interference of these two amplitudes. Since $f$ and $g$ are functions of $\theta$ this discussion applies for every scattering angle. If we are interested in $f$ and $g$ at all angles, then they are best expressed as sums of partial-wave amplitudes and the experimental data analyzed in terms of phase shifts.

The possibility of a direct determination of the scattering matrix from the experimentally measured quantities is essential at high energies, where a phase shift analysis is hindered by a large number of 
statfs and by the need of taking into account the influence of inelastic processes. In a phase shift analysis of the $\pi-N$ scattering data, further simplifications are obtained by using the requirements of isospin invariance of strong interactions.

All the relations given above so far and also in the next section are based only on invariance under rotations and parity. This means that all the foregoing pertains also to inelastic reactions of the type $0+\frac{1}{2}+0+\frac{1}{2}$, provided the total intrinsic parity of the initial and final particles are the same. 


\subsection{Phase Shift Analysis}

The incident particles, which are assumed to have spin $\frac{1}{2}$, are repiesented by a wave function of the form

$$
\begin{gathered}
e^{i k z}\left|x_{1}\right\rangle=e^{i k z}\left(\begin{array}{l}
a \\
b
\end{array}\right), \\
\left\langle x_{i} \mid x_{i}\right\rangle=a^{*} a+b^{*} b=1 .
\end{gathered}
$$

We look for asymptotic solutions of the Schrodinger equation which have the form

$$
e^{i k z}\left|x_{i}\right\rangle+h(\theta, \phi) \frac{e^{i k r}}{r}
$$

where the scattering amplitude $h(\theta, \phi)$ is a two-component spinor. The linearity of the Schrodingar equation allows us to construct the solution (1.79) from two particular solutions which correspond to $\left|x_{1}\right\rangle=|+\rangle$ and $\left|x_{1}\right\rangle=\mid \rightarrow$. These two special cases describe incident beams which are polarized along the direction of the initial momentum and opposite to it. We are thus led to look for two solutions of the asymptoti : form

$$
\begin{aligned}
& \left.\left|\psi_{1}==e^{i k z}\right|+\right\rangle+\left(M_{11}|+\rangle+M_{21} \mid \rightarrow\right) \frac{e^{i k r}}{r} \\
& \left|Y^{\prime}\right\rangle=e^{i k z}\left|\rightarrow+\left(M_{12}|+\rangle+M_{22}|-\rangle\right) \frac{e^{i k r}}{r} .\right.
\end{aligned}
$$

These equations are a precise definition of $M$. The quantities in parentheses are the appropriate scattering amplitudes. Multiplying $(1.80 a)$ by $a$, and $(1.80 b)$ by $b$, and adding the two equations, we obtain the general solution

$$
e^{i k z}\left(\begin{array}{l}
a \\
b
\end{array}\right)+\left(\begin{array}{ll}
M_{11} & M_{12} \\
M_{21} & M_{22}
\end{array}\right)\left(\begin{array}{l}
a \\
b
\end{array}\right) \frac{e^{i k r}}{r}=\left(e^{i k z}+M \frac{e^{i k r}}{r}\right)\left|x_{i}\right\rangle .
$$

Here M stands for the $2 \times 2$ scattering matrix:

$$
M=\left(\begin{array}{ll}
M_{11} & M_{12} \\
M_{21} & M_{22}
\end{array}\right)
$$

$M$ depends on the angles $\theta$ and $\phi$, and on the momentum $k$. The scattering 
problem is solved if $M$ can be determined as a function of these variables. The incident waves in ( $1.80 \mathrm{a})$ and $(1.80 \mathrm{~b})$ are eigenstates of the $z$-component of the total angular momentum $J_{z}$ with eigenvalues $+1 / 2$ and $-1 / 2$, respectively. Since the operator $J_{z}$ leaves the radial dependence of the scattered wave unchanged, the solutions (1.80) must both be eigenfunctions of $\mathrm{J}_{z}$. By requiring that

$$
\begin{aligned}
& \mathrm{J}_{\mathrm{z}}\left|\psi_{\mathrm{l}}\right\rangle=+\frac{1}{2}\left|\psi_{1}\right\rangle, \\
& \mathrm{J}_{\mathrm{z}}\left|\psi_{2}\right\rangle=-\frac{1}{2}\left|\psi_{2}\right\rangle,
\end{aligned}
$$

and noting that

$$
\begin{aligned}
& J_{z}=l_{z}+s_{z} \\
& =\frac{1}{i} \frac{\partial}{\partial \phi}+\frac{1}{2} \sigma_{z} \\
& =\left(\begin{array}{cc}
\frac{1}{1} \frac{\partial}{\partial \phi}+\frac{1}{2} & 0 \\
0 & \frac{1}{1} \frac{\partial}{\partial \phi}-\frac{1}{2}
\end{array}\right),
\end{aligned}
$$

and using $z=r \cos \theta$, from $(1.80)$ we find

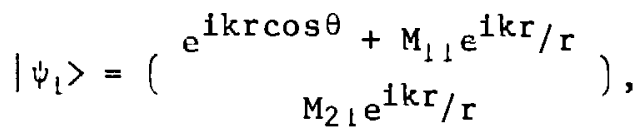

$$
\begin{aligned}
& \left|\psi_{2}\right\rangle=\left(\begin{array}{c}
M_{12} e^{i k r} / r \\
e^{i k r \cos \theta}+M_{22} e^{i k r} / r
\end{array}\right) .
\end{aligned}
$$

Substituting $(1.84)$ and $(1.85 a)$ into (1.83a) we have

$$
\begin{aligned}
\frac{\partial}{\partial \phi} M_{11} & =0, \\
\frac{1}{i} \frac{\partial}{\partial \phi} M_{21} & =M_{21} .
\end{aligned}
$$

Similarly substituting eqs. ( 1.84$)$ and (1.85b) into (1.83b) we have

$$
\begin{aligned}
\frac{1}{1} \frac{\partial}{\partial \phi} M_{12} & =-M_{12}, \\
\frac{\partial}{\partial \phi} M_{22} & =0 .
\end{aligned}
$$


From eqs. (1.86a) and (1.86d) it is seen that $M_{1} \downarrow$ and $M_{22}$ are independent of $\phi$ and can be functions of $\theta$ only, and from eqs. (1.86b) and $(1.86 \mathrm{c})$ it follows that the off-diagonal elements of the scattering matrix have the form

$$
\begin{aligned}
& M_{12}=e^{-i \phi_{1}} \text { function of } \theta, \\
& M_{21}=e^{+i \phi} \text { function of } \theta .
\end{aligned}
$$

Furthermore, the interaction is invariant under a reflection with respect to any coordinate plane. The operator for reflection in the $y-z$ plane is $\pi_{x} \sigma_{x}$, where $\pi_{x}$ simply changes $x$ to $-x$, and $\sigma_{x}$ has the effect

$$
\sigma_{x}|+\rangle=|-\rangle, \sigma_{x}|-\rangle=|+\rangle \text {. }
$$

Under this operation the incident wave $e^{i k z} \mid+>$ changes into $e^{i k z} \mid->$ and $\mathrm{e}^{i k r} / \mathrm{r}$ is invariant. Hence $(1.80 \mathrm{a})$ must go over into (1.80b). In terms of spherical coordinates $\pi_{x}$ has the effect of changing $\phi$ into $\pi-\phi$. It follows from this and $(1.87)$ that

$$
\begin{gathered}
M_{11}=M_{22}=f(\theta) \\
M_{21}(\theta,-\phi)=-M_{12}(\theta, \phi)=i g(\theta) e^{-i \phi}
\end{gathered}
$$

where we have introduced a factor of $i$ to get agreement with our previous results. * Consequently we may write

$$
\begin{aligned}
M & =\left(\begin{array}{cc}
f(\theta) & -i g(\theta) e^{-i \phi} \\
i g(\theta) e^{i \phi} & F(\theta)
\end{array}\right) \\
& =f(\theta)+g(\theta)\left(\sigma_{y} \cos \phi-\sigma_{x} \sin \phi\right) .
\end{aligned}
$$

The unit vector $\hat{\mathrm{n}}=(-\sin \phi, \cos \phi, 0)$ is the normal to the plane of ₹ If instead of eq. $(1.88 b)$ we use $M 2 I(\theta,-\phi)=-M_{12}(\theta, \phi)=-g(\theta) e^{-i \phi}$ we find Sakurai's form: $M=f(\theta)+i g(\theta) \vec{\sigma} \cdot \hat{n}$ and if we use $M_{2 l}(\theta,-\phi)=$ $-M_{12}(\theta, \phi)=g(\theta) e^{-i \phi}$ we end up with Williams s $s^{15}$ form $M=f(\theta)-i g(\theta)+\hat{\sigma} \cdot \hat{n}$. 
scattering. Hence, we conclude that the scattering matrix has the forin

$$
M=f(\theta)+g(\theta) \vec{\sigma} \cdots
$$

This is as much as we can infer about the scattering mastrix from the invarlance properties of the interaction. The asyuptotic form of the wave function takes the form

$$
\begin{gathered}
\left|\psi_{1}\right\rangle=e^{i k z}|+\rangle+\left(f(\theta)|+\rangle+\lg (\theta) e^{i \phi}|-\rangle\right) \frac{e^{i k r}}{r}, \\
\left|\psi_{2}\right\rangle=e^{i k z}\left|\rightarrow+\left(-1 g(\theta) e^{-i \phi}|+\rangle+f(\theta) \mid->\right) \frac{e^{i k r}}{r} .\right.
\end{gathered}
$$

We start with

$$
\left|\psi_{m}\right\rangle=e^{i k z}\left|x_{m^{\prime}}\right\rangle+\left(f(\theta)\left|x_{m}\right\rangle+g(\theta, \phi)\left|x_{-m}\right\rangle\right) \frac{e^{i k r}}{r}
$$

where the incoming wave $e^{i k z}\left|x_{m}\right\rangle$ can be expanded as:

$$
e^{i k z}=\sum_{\ell} i^{\ell}(2 \ell+1) j_{\ell}(k r) P_{\ell}(\cos \theta) .
$$

The asymptotic form of the Bessel function is

$$
\mathrm{j}_{\ell}(\mathrm{kr}) \stackrel{\mathrm{r}+\infty}{\longrightarrow} \frac{1}{\mathrm{kr}} \sin (\mathrm{kr}-\ell \pi / 2),
$$

and

$$
\mathrm{Y}_{\ell}^{0}(\theta, \phi)=\sqrt{(2 \ell+1) / 4 \pi} \mathrm{P}_{\ell}(\cos \theta)
$$

Substituting eqs. $(1.94)$ and $(1.95)$ into (1.93) we find

$$
\begin{aligned}
\mathrm{e}^{i \mathrm{kz}} \stackrel{\mathrm{r \rightarrow \infty} \longrightarrow}{\longrightarrow} \sum_{\ell} \sqrt{4 \pi(2 \ell+1)} \mathrm{i}^{\ell} \frac{1}{\mathrm{kr}} \sin (\mathrm{kr}-\ell \pi / 2) \mathrm{Y}_{\ell}^{O}(\theta, \phi) \\
=\sum_{\ell} \sqrt{4 \pi(2 \ell+1)} \frac{1}{2 i \mathrm{kr}}\left(\mathrm{e}^{i k r}-(-1)^{\ell} \mathrm{e}^{-i k r}\right) \mathrm{Y}_{\ell}^{O}(\theta, \phi) .
\end{aligned}
$$


We also expand $f(\theta)$ and $g(\theta, \phi)$ in spherical harmontes

$$
\begin{aligned}
\mathrm{f}(\theta) & =\sum_{\ell=0}^{\infty} \mathrm{f}_{\ell} \mathrm{Y}_{\ell}^{O}(\theta, \phi), \\
\mathrm{g}(\theta, \phi) & =\sum_{\ell=1}^{\infty} \mathrm{g}_{\ell} \mathrm{Y}_{\ell}^{2 \mathrm{~m}}(\theta, \phi) .
\end{aligned}
$$

We obtain 22

$$
\begin{aligned}
& \mathrm{f}_{\ell}=\frac{\sqrt{4 \pi /(2 \ell+1)}}{\mathrm{k}}\left[(\ell+1) \mathrm{a}_{\ell, \ell+1 / 2}+\ell \mathrm{a}_{\ell, \ell-1 / 2}\right], \\
& \mathrm{g}_{\ell}=\frac{\sqrt{4 \pi /(2 \ell+1)}}{\mathrm{k}} \sqrt{\ell(\ell+1)}\left[\mathrm{a}_{\ell, \ell+1 / 2}-\mathrm{a}_{\ell, \ell-1 / 2}\right] .
\end{aligned}
$$

The partial wave scattering amplitude, with spin taken into account is defined as

$$
a_{\ell, j}=\frac{1}{2 j}\left(e^{2 i \delta_{\ell, j}}-1\right)=e^{1 \delta_{\ell, j}} \sin \delta_{\ell, j} \cdot
$$

If the initial proton target is unpolarized and we sum over the recoil proton spin directions, the differential cross section becomes

$$
\begin{aligned}
\left(\frac{\mathrm{d} \sigma}{\mathrm{d} \Omega}\right)_{\text {unpol }} & =|\mathrm{f}(\theta)|^{2}+|\mathrm{g}(\theta, \phi)|^{2} \\
& =\left|\sum_{\ell} \mathrm{f}_{\ell} \mathrm{Y}_{\ell}^{\mathrm{O}}\right|^{2}+\left|\sum_{\ell} \mathrm{g}_{\ell} \mathrm{Y}_{\ell}^{2 \mathrm{~m}}\right|^{2} .
\end{aligned}
$$

This allows us to express the differential cross section in terms of the scattering amplitudes $a_{\ell, j}$. At low energies only phase shifts for small values of $\ell$ contribute. Experimentally, we krow that the angular distribution $1 \mathrm{e}$ not isotropic. Therefore it is not enough to include only s-waves in our analysis. At the region of this experiment we must consider $\mathrm{s}, \mathrm{p}, \mathrm{d}, \mathrm{f}$, and $\mathrm{g}$ waves which makes the analysis very complicated. As an illustration of the ideas of a partial wave analysis consider the sase of s-waves and p-waves only and neglect all higher partial waves

$$
\left(\frac{d u}{d \Omega}\right)_{\text {Unpol }}=\left|f_{O} Y_{O}^{0}+f_{L} Y_{L}^{0}\right|^{2}+\left|g_{L} Y_{L}^{L}\right|^{2} \text {. }
$$


From ( 1.98$)$ we find

$$
\begin{aligned}
& \mathrm{f}_{0}=\frac{\sqrt{4 \pi}}{\mathrm{k}} \mathrm{a}_{0,1 / 2}, \\
& \mathrm{f}_{1}=\frac{\sqrt{4 \pi / 3}}{\mathrm{k}}\left(2 \mathrm{a}_{1,3 / 2}+\mathrm{a}_{1,1 / 2}\right), \\
& \mathrm{g}_{1}=\frac{\sqrt{8 \pi / 3}}{\mathrm{k}}\left(\mathrm{a}_{1,3 / 2}-\mathrm{a}_{1,1 / 2}\right) .
\end{aligned}
$$

Introducing all these in eq. (1.101) together with the explicit Formulas for the spherical harmonics, the unpolarized differential cross section becomes

$$
\frac{\mathrm{d} \sigma}{\mathrm{d} \Omega}=\frac{1}{\mathrm{k}^{2}}\left[\left|\mathrm{a}_{0,1,2}+\left(2 \mathrm{a}_{1,3 / 2}+\mathrm{a}_{1,1 / 2}\right) \cos \theta\right|^{2}+\left|\mathrm{a}_{1,3 / 2}-\mathrm{a}_{1,1 / 2}\right|^{2} \sin ^{2} \theta\right] \text {. }
$$

This is a second-order polynomial in $\cos \theta$. The quadratic term in this polynomial is made up of p-waves only. It can be obtained from scattering in a single state only if $\mathrm{J}=3 / 2$. The linear term is the interference term between $p$-waves and s-waves and it is absent if there were only p-state scattering. The constant term gets contributions from both p-waves and s-waves.

So far we have not mentioned the isospin quantum number. Our discussion was based on the asymptotic form of the wave function. In reality, we have co make the same argument separately for each reaction $\pi^{+} p \rightarrow \pi^{+} p, \pi^{-} p \rightarrow \pi^{-} p$, and $\pi^{-} p_{p \rightarrow \pi^{\circ}} n$. Since the nucleon is an isospinor, $I_{N}=1 / 2$, and pion an isovector, $I_{\pi}=1$, and isospins add vectorially like angular momentum the pion-nucleon system can have total isospin $I_{\text {total }}=1 / 2$, or $3 / 2$. The $\pi^{+} p$ system has $I_{3}=+3 / 2$ and is therafore described by a pure $I=3 / 2$ amplitude, while the $\pi^{-} p$ has $I_{3}=-1 / 2$ and 
therefore $I=3 / 2$ or $1 / 2$. Usually we characterice a state with isospin $I$ and its third component $I_{3} ;\left|I_{1} I_{3}\right\rangle$. Thus

$$
\begin{aligned}
& \left|\pi^{+}, \mathrm{p}\right\rangle=|3 / 2,3 / 2\rangle \\
& \left|\pi^{-}, \mathrm{p}\right\rangle=\frac{1}{\sqrt{3}}(|3 / 2,-1 / 2\rangle-\sqrt{2}|1 / 2,-1 / 2\rangle), \\
& \left|\pi^{0}, \mathrm{n}\right\rangle=\frac{1}{\sqrt{3}}(\sqrt{2}|3 / 2,-1 / 2\rangle+|1 / 2,-1 / 2\rangle) .
\end{aligned}
$$

The differential cross section is equal to the square of the matrix element connecting initial and final states, i.e.

$$
\frac{\mathrm{d} \sigma}{\mathrm{d} \Omega}=\rho\left|\left\langle\psi_{\mathrm{f}}|\mathrm{M}| \psi_{\mathbf{i}}\right\rangle\right|^{2}
$$

where $p$ is the density of final staices.

The hypothesis of charge independence states that the orientation of the third axis in isospin space has no physical significance. This means that scattering depends only on $I$ and not on $I_{3}$. In other words there is no operator connecting initial and final states of different isospin. Thus the two amplitudes

$$
\begin{aligned}
& a^{1 / 2}=\left\langle\psi_{f}\left(\frac{1}{2}\right)|M| \psi_{i}\left(\frac{1}{2}\right)\right\rangle, \\
& a^{3 / 2}=\left\langle\psi_{f}\left(\frac{3}{2}\right)|M| \psi_{i}\left(\frac{3}{2}\right)\right\rangle,
\end{aligned}
$$

are sufficient to characterize the scattering. We find

$$
\begin{array}{ll}
\frac{\mathrm{d} \sigma^{+}}{\mathrm{d} \Omega}=\left|a^{3 / 2}\right|^{2} & \text { for } \pi^{+}+p+\pi^{+}+p \\
\frac{\mathrm{d} \sigma^{-}}{\mathrm{d} \Omega}=\frac{1}{9}\left|a^{3 / 2}+2 a^{1 / 2}\right|^{2} & \text { for } \pi^{-}+p \rightarrow \pi^{-}+p \\
\frac{\mathrm{d} \sigma^{0}}{\mathrm{~d} \Omega}=\frac{2}{9}\left|a^{3 / 2}-a^{1 / 2}\right|^{2} & \text { for } \pi^{-}+p \rightarrow \pi^{0}+n
\end{array}
$$

Thus owing to isospin conservation there are only two independent amplitudes, and we need only double our parameters to describe the $\pi-N$ 
system. Six independent amplitudes are sufficient to describe both the $\pi-p$ and $\pi-n$ systems. " This means that each amplitude $a_{\ell, j}$ appears twice, once for $I=3 / 2$ and once for $I=1 / 2$. To distinguish them we add the isospin as an upper index and wrice $a_{\ell, j}^{I}$. The actual partial wave scattering amplitude for the three different scattering processes $\pi^{ \pm} p \rightarrow \pi^{ \pm} p$ and $\pi^{-} p+\pi^{\circ} n$ can then be written

$$
\begin{array}{ll}
a^{+}=a_{\ell, j}^{3 / 2} & \text { for } \pi^{+}+p \rightarrow \pi^{+}+p, \\
a^{-}=\frac{1}{3}\left(a_{\ell, j}^{3 / 2}+2 a_{\ell, j}^{1 / 2}\right) & \text { for } \pi^{-}+p \rightarrow \pi^{-}+p, \\
a^{0}=\frac{\sqrt{2}}{3}\left(a_{\ell, j}^{3 / 2}-a_{\ell, j}^{1 / 2}\right) & \text { for } \pi^{-}+p \rightarrow \pi^{0}+n .
\end{array}
$$

Thus from isospin invariance

$$
a^{+}=a^{-}+\sqrt{2} a^{0}
$$

The arguments leading to eq. (1.79) and the derivation of (1.98) are valid provided the potential goes to zero more rapidly than $1 / \mathrm{r}$ at large $r$, and are therefore not valid in the case when the potential includes a Coulomb term. When the Coulomb potential is included, it is still possible to make a partial wave expansion of the scattering. The Coulomb amplitude is 23

where

$$
f_{c}(\theta)=-\frac{\gamma}{2 k \sin ^{2}(\theta / 2)} e^{-1 \gamma \log \sin ^{2}\left(\frac{\theta}{2}\right)+2 i \sigma_{0}}
$$

$$
e^{2 i \sigma_{\ell}}=\frac{\Gamma(\ell+1+i \gamma)}{\Gamma(\ell+1-i \gamma)} \quad \text { Coulomb phase shift }
$$

and

$$
Y=\frac{Z_{1} Z_{2} e^{2}}{h v}=\frac{\mu Z_{1} Z_{2} e^{2}}{h^{2} k}, \quad \mu=\text { reduced mass. }
$$

FIF isospin were not conserved, we would need nine independent amplitudes to describe the $\pi-p$ system, and if charge symmetry were not valid, we would need another nine for the $\pi-n$ system. 
We can take the Coulomb effect into account by adding the Coulomb amplitude to the non spin-flip amplitude (neglecting the distortion of the nuclear potential by the Coulomb potential), since Coulomb scattering does not involve spin flip

$$
\mathrm{f}_{\text {total }}(\theta)=\mathrm{f}_{\mathrm{c}}(\theta)+\mathrm{f}(\theta)
$$

and thus

$$
\left(\frac{\mathrm{d} \sigma}{\mathrm{d} \Omega}\right)_{\text {unpol }}=\left|\mathrm{f}_{\mathrm{c}}(\theta)+\mathrm{F}(\theta)\right|^{2}+|\mathrm{g}(\theta, \phi)|^{2}
$$

The differential cross section corresponding to the Coulomb interaction 1s peaked in the forward direction. Consequently, it is far from isotropic and keeping $\ell=0,1$ terms only and neglecting $\ell>2$ approximation is not justified.

Let us consider the large angle approximation where $F_{c}(\theta)<<f(\theta)$, and neglect $f_{c}(\theta)$. In eq. (1.102), we can replace $\sin ^{2} \theta$ by $1-\cos ^{2} \theta$, and obtain

$$
\begin{aligned}
\left(\frac{d \sigma}{d \Omega}\right)_{\text {unpol }}= & \frac{1}{{ }^{2}}\left\{\left|a_{0,1 / 2}\right|^{2}+\left|a_{1,3 / 2}-a_{1,1 / 2}\right|^{2}+\right. \\
& 2 \operatorname{Re}\left[a_{0,1 / 2}{ }^{*}\left(2 a_{1,3 / 2}+a_{1,1 / 2}\right) \cos \theta\right]+ \\
& \left.\left(\left|2 a_{1,3 / 2}+a_{1,1 / 2}\right|^{2}-\left|a_{1,3,2}-a_{1,1 / 2}\right|^{2}\right) \cos ^{2} \theta\right\} .
\end{aligned}
$$

Hence

where

$$
\left(\frac{\mathrm{d} \sigma}{\mathrm{d} \Omega}\right)_{\text {unpol }}=\frac{1}{\mathrm{k}^{2}}\left(\mathrm{~A}+\mathrm{B} \cos \theta+\cos ^{2} \theta\right),
$$

$$
\begin{aligned}
& A=\left|a_{0,1 / 2}\right|^{2}+\left|a_{1,3 / 2}-a_{1,1 / 2}\right|^{2}, \\
& B=2 \operatorname{Re}\left[a_{0,1 / 2}^{*}\left(2 a_{1,3 / 2}+a_{1,1 / 2}\right)\right], \\
& C=3\left[\left|a_{1,3 / 2}\right|^{2}+2 \operatorname{Re}\left(a_{1,3,2} * a_{1,1 / 2}\right)\right] .
\end{aligned}
$$


We can write for the three unpolarized $\pi^{ \pm}-p$ scattering processes

$$
\begin{aligned}
& \frac{d \sigma}{d \Omega}\left(\pi^{+} p \rightarrow \pi^{+} p\right)=\frac{1}{k^{2}}\left(A^{+}+B^{+} \cos \theta+C^{+} \cos ^{2} \theta\right), \\
& \frac{d \sigma}{d \Omega}\left(\pi^{-} p+\pi^{-} p\right)=\frac{1}{k^{2}}\left(A^{-}+B^{-} \cos \theta+C^{-} \cos ^{2} \theta\right), \\
& \frac{d \sigma}{d \Omega}\left(\pi^{-} p \rightarrow \pi^{\circ} n\right)=\frac{1}{k^{2}}\left(A^{0}+B^{\circ} \cos \theta+C^{0} \cos ^{2} \theta\right) .
\end{aligned}
$$

For $\pi^{+}$p elastic scattering from eq. (1.107a) and (1.114) we find

$$
\begin{aligned}
& \mathrm{A}^{+}=\left|\mathrm{a}_{0,1 / 2}^{3 / 2}\right|^{2}+\left|\mathrm{a}_{1,3 / 2}^{3 / 2}-a_{1,1 / 2}^{3 / 2}\right|^{2}, \\
& \mathrm{~B}^{+}=2 \operatorname{Re}\left[\mathrm{a}_{0,1 / 2}^{3 / 2}{ }^{*}\left(2 \mathrm{a}_{1,3 / 2}^{3 / 2}+\mathrm{a}_{1,1 / 2}^{3 / 2}\right)\right], \\
& \mathrm{C}^{+}=3\left[\left|\mathrm{a}_{1,3 / 2}^{3 / 2}\right|^{2}+2 \operatorname{Re}\left(\mathrm{a}_{1,3 / 2}^{3 / 2} * \mathrm{a}_{1,1 / 2}^{3 / 2}\right)\right] .
\end{aligned}
$$

Similar but more complicated expressions involving $a_{\ell, j}^{3 / 2}$ and $a_{\ell, j}^{1 / 2}$, can be derived for $A^{-}, B^{-}, C^{-}$, and $A^{\circ}, B^{\circ}$, and $C^{\circ}$ by inserting eqs. $(1.107 b, c)$ into $(1.114)$.

Let us row express the scattering amplitudes in terms of the corresponding phase shifts. We adopt the standard notation for a phase shift: $\delta_{2 I, 2 J}$ is the phase shift for the state with isospin $I$ and angular momentum $J$. For $s$-waves, the angular momentum index $J$ is omitted, but it is understood that $J=1 / 2$. In this notation

$$
a_{\ell, j}^{I}=\frac{1}{2 i}\left(e^{2 i \delta} 2 I, 2 J-1\right)=e^{i \delta} 2 I, 2 J \sin \delta 2 I, 2 J
$$

For $\pi^{+}$-p elastic scattering we have $I=3 / 2$, and thus

$$
\begin{aligned}
& a_{0, L / 2}^{3 / 2}=e^{i \delta 3} \sin \delta_{3}, \\
& a_{1,1 / 2}^{3 / 2}=e^{i \delta 31} \sin \delta_{1}, \\
& a_{1,3 / 2}^{3 / 2}=e^{i \delta 33} \sin \delta_{3} .
\end{aligned}
$$


Expressing (1.116) in terms of phase shifts, we find

$$
\begin{aligned}
& A^{+}=\sin ^{2} \delta_{3}+\sin ^{2}\left(\delta_{33}-\delta_{31}\right), \\
& \mathrm{B}^{+}=2 \sin \delta_{3}\left[2 \sin \delta_{33} \cos \left(\delta_{33}-\delta_{3}\right)+\sin \delta_{31} \cos \left(\delta_{31}-\delta_{3}\right)\right] \text {, } \\
& C^{+}=3\left[\sin ^{2} \delta_{33}+2 \sin \delta_{31} \sin \delta_{33} \cos \left(\delta_{33}-\delta_{31}\right)\right] \text {. }
\end{aligned}
$$

Let us now assume that only $\delta_{33} \neq 0$. Then we find

$$
\begin{array}{lll}
\mathrm{A}^{+}=\left|\mathrm{a}_{1,3 / 2}^{3 / 2}\right|^{2}=\sin ^{2} \delta_{33}, & \mathrm{~B}^{+}=0, & \mathrm{C}^{+}=3 \sin ^{2} \delta_{33} \\
\mathrm{~A}^{-}=\frac{1}{9}\left|\mathrm{a}_{1,3 / 2}^{3 / 2}\right|^{2}=\frac{1}{9} \sin ^{2} \delta_{33}, & \mathrm{~B}^{-}=0, & \mathrm{C}^{-}=\frac{1}{3} \sin ^{2} \delta_{33} \\
\mathrm{~A}^{\mathrm{O}}=\frac{2}{9}\left|\mathrm{a}_{1,3,2}^{3 / 2}\right|^{2}=\frac{2}{9} \sin ^{2} \delta_{33}, & \mathrm{~B}^{\mathrm{O}}=0, & \mathrm{C}^{\mathrm{O}}=\frac{2}{3} \sin ^{2} \delta_{33}
\end{array}
$$

Inserting these into $(1.115)$ we find

$$
\begin{aligned}
& \frac{\mathrm{d} \sigma^{+}}{\mathrm{d} \Omega}=\frac{\sin ^{2} \delta_{33}}{\mathrm{k}^{2}}\left(1+3 \cos ^{2} \theta\right) \\
& \frac{\mathrm{d} \sigma^{-}}{\mathrm{d} \Omega}=\frac{\sin ^{2} \delta 33}{9 \mathrm{k}^{2}}\left(1+3 \cos ^{2} \theta\right) \\
& \frac{\mathrm{d} \sigma^{\circ}}{\mathrm{d} \Omega}=\frac{2 \sin ^{2} \delta_{33}}{9 k^{2}}\left(1+3 \cos ^{2} \theta\right)
\end{aligned}
$$

Thus if on $1 y \quad \delta_{33} \neq 0$

$$
\frac{\mathrm{d} \sigma^{+}}{\mathrm{d} \Omega}: \frac{\mathrm{d} \sigma^{-}}{\mathrm{d} \Omega}: \frac{\mathrm{d} \sigma^{0}}{\mathrm{~d} \Omega}=9: 1: 2 .
$$

Let us norj go back to eq. (1.43) which for a transversely polarized target could be written as

$$
I( \pm)=I_{0}\left(1 \pm P_{i} A_{N}\right)
$$

or

$$
\begin{array}{ll}
I^{+}( \pm)=I_{0}^{+}\left(1 \pm P_{i} A_{N}^{+}\right) & \text {for } \pi^{+}+p \rightarrow \pi^{+}+p \\
I^{-}( \pm)=I_{0}^{-}\left(1 \pm P_{i} A_{N}^{-}\right) & \text {for } \pi^{-}+p \rightarrow \pi^{-}+p \\
I^{O}( \pm)=I_{0}^{0}\left(1 \pm P_{i} A_{N}^{0}\right) & \text { for } \pi^{-}+p+\pi^{0}+n
\end{array}
$$


and from eas. $(1.106)$ and $\left(1.10^{7}\right)$

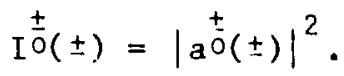

From eq. $(1.108)$ we see that

$$
a^{+}( \pm)=a^{-}( \pm)+\sqrt{2} a^{0}( \pm) \text {. }
$$

This implies that the three amplitudes $\mathrm{a}^{+}, \mathrm{a}^{-}$, and $\mathrm{a}^{0}$ form a triangle in the complex plane. Since the length of one side in a triangle is always smaller than the sum of the lengths of two other sides and larger than their difference, we have

$$
\left|a^{+}( \pm)\right|-\left|a^{-}( \pm)\right| \leqslant \sqrt{2}\left|a^{0}( \pm)\right|<\left|a^{+}( \pm)\right|+\left|a^{-}( \pm)\right|
$$

or, in terms of measured differential cross sections and analyzing powers

$$
\frac{1}{2}\left(\sqrt{\mathrm{I}^{+}( \pm)}-\sqrt{\mathrm{I}^{-}( \pm)}\right)^{2}<\mathrm{I}^{\circ}( \pm)<\frac{1}{2}\left(\sqrt{\mathrm{I}^{+}( \pm)}+\sqrt{\mathrm{I}^{-}( \pm)}\right)^{2}
$$

while for unpolarized differential cross sections

$$
\frac{1}{2}\left(\sqrt{I_{0}^{+}}-\sqrt{I_{0}^{-}}\right)^{2} \leqslant I_{0}^{0} \leqslant \frac{1}{2}\left(\sqrt{I_{0}^{+}}+\sqrt{I_{0}^{-}}\right)^{2}
$$

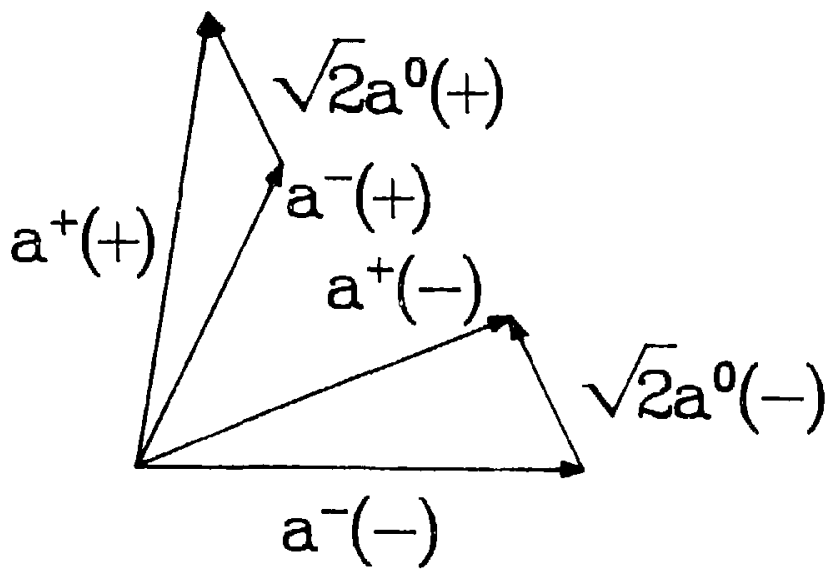

Figure 1.6. The amplitudes $a^{+}, a^{-}$, and $a^{\circ}$ form a triangle in the complex plane. 
The analyzing power could also be expressed in terms of phase shifts. From tis definition we have

$$
I_{0} A_{N}(\theta)=\mathrm{fg}^{\star}+\mathrm{f}^{\star} \mathrm{g}=2 \operatorname{Re}\left(\mathrm{fg}^{\star}\right),
$$

where $f(\theta)$ is

$$
\begin{aligned}
f(\theta) & =f_{0} Y_{0}^{0}(\theta, \phi)+f_{L} Y_{1}^{0}(\theta, \phi) \\
& =\frac{1}{k}\left[a_{0,1 / 2}+\left(2 a_{1,3 / 2}+a_{1,1 / 2}\right) \cos \theta\right],
\end{aligned}
$$

and for $\pi^{+}$p elastic scattering $f(\theta)$ in terms of the phase shifts is

$$
f(\theta)=\frac{1}{k}\left[e^{1 \delta_{3}} \sin \delta_{3}+\left(2 e^{i \delta_{33}} \sin \delta_{33}+e^{i \delta_{3 !} \sin \delta_{3 !}}\right) \cos \theta\right] .
$$

While the spin-flip amplitude is

$$
\begin{aligned}
& \qquad \begin{aligned}
g(\vartheta, \phi) & =\operatorname{gtY} Y_{1}^{t}(\theta, \phi) \\
& =\frac{-1}{k}\left(a_{1,3 / 2}-a_{1,1 / 2}\right) \sin \theta e^{i \phi} .
\end{aligned} \\
& \text { Comparing }(1.91 a) \text { with }(1.92) \text { we find }(m=+1 / 2) \\
& g(\theta, \phi)=i g(\theta) e^{i \phi} .
\end{aligned}
$$

Therefore $g(\theta)$ is

$$
g(\theta)=\frac{i}{k}\left(a_{1,3 / 2}-a_{1,1 / 2}\right) \sin \theta,
$$

and for $\pi^{+}$p elastic scattering $g(\theta)$ in terms of the $s, p$ phase shifts becomes

$$
g(\theta)=\frac{1}{k}\left(e^{1 \delta_{33}} \sin \delta_{33}-e^{1 \delta_{3 \perp}} \sin \delta_{31}\right) \sin \theta .
$$

Substituting eqs. (1.127) and (1.130) into (1.126) we obtain

$$
I_{0} A_{N}(\theta)=\frac{-2}{k^{2}} \operatorname{Im}\left\{\left[a_{0,1 / 2}+\left(a_{1,3 / 2}+a_{1,1 / 2}\right) \cos \theta\right]\left(a_{1,3 / 2}^{*}-a_{1,1 / 2}\right) \sin \theta\right\} \text {. }
$$

The analyzing power for $\pi^{+} p$ elastic scattering in terms of the $s, p$ phase shifts is

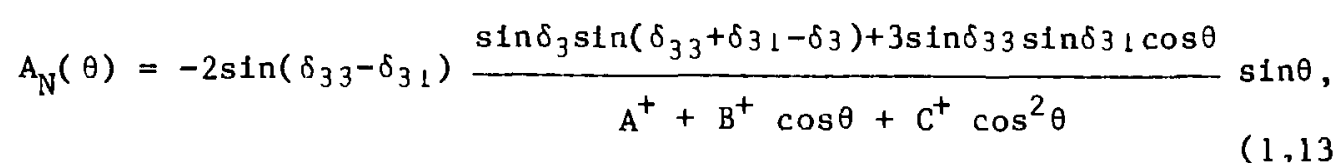


where $\mathrm{A}^{+}, \mathrm{B}^{+}$, and $\mathrm{C}^{+}$are given by eqs. (1.116).

If we retain only $\ell=0,1$ terms, fron eq. (1.130) or $(1.131)$ it is seen that $g(\theta)$ is proportional to $\sin \theta$. This statement is true at any order. In $(1.9 \mathrm{la}) \mathrm{m}=+1 / 2$, and from $(1.96 \mathrm{~b})$ we $f$ ind

$$
g(\theta, \phi)=\sum_{\ell} g_{\ell} Y_{\ell}^{l}(\theta, \phi),
$$

where

$$
Y_{\ell}^{1}(\theta, \phi)=-\left[\frac{2 \ell+1}{4 \pi} \frac{(\ell-1) !}{(\ell+1) !}\right]^{1 / 2} P_{\ell}^{1}(\cos \theta) e^{i \phi}
$$

and

$$
\mathrm{P}_{\ell}^{!}(\cos \theta)=\sin \theta \frac{\mathrm{dP}_{\ell}}{\mathrm{d}(\cos \theta)} .
$$

The relation between $g(\theta, \phi)$ and $g(\theta)$ is given by eq. (1.129), and thus It is seen that $g(\theta)$ is proportional to $\sin \theta$. Therefore

$$
g(\theta)=0 \text { at } \theta=0 \text {, or } \pi
$$

Since

we find that

$$
A_{N}(S)=\frac{2 \operatorname{Re}\left(\mathrm{fg}^{*}\right)}{|\mathrm{f}|^{2}+|\mathrm{g}|^{2}}
$$

$$
A_{N}(\theta)=0 \text { at } \theta=0 \text {, or } \pi
$$

The total angular momentum $\vec{J}$ is compounded from the orbital angular momentum $\vec{l}$ and the spin of the nucleon $\vec{s}: \vec{J}=\vec{\imath}+\vec{s}$, therefore $j=\ell \pm 1 / 2$ or $\ell=j \pm 1 / 2$. Conservation of parity requires $(-)^{\ell}=(-)^{\ell^{-}}$. Because $\ell$ and $\ell^{-}$must be $j \pm 1 / 2$, we see that $\ell=\ell^{-}$. We can talk about $\ell$ instead of parity. For a given $j$, only two states with $\ell$ values $j+1 / 2$ and $j-1 / 2$ are possible. These states, differing $1 \mathrm{n} \ell$ by 1 , have opposite parfty. States of the same $j$ but different $\ell$ do not mix. This means that if we interchange partial-wave amplitudes between the 
two states of different $\ell$ (and thus opposite parity) but of the same $j$, then there is no change in the angular distribution. Under these interchanges

$$
\mathrm{f}^{-}+\mathrm{ig}^{-}
$$

the differential cross section does not change but the analyzing power changes sign. This is called the Minami ${ }^{24}$ ambiguity and is resolved by a measurement of the analyzing power. A more general form of the Minami ambiguity is

$$
f^{-\star}+i g^{-}
$$

This has no effect on either the differential cross section or the analyzing power. This ambiguity can be resolved by measuring the $A$ or R parameter.

The Yang ambiguity arises from charging the sign of either $f$ or $g$ :

$$
f \rightarrow-f \text { and } g+g \text { or } f+f \text { and } g+-g
$$

Again under these changes the differential cross section section remains unchanged, but the analyzing power changes sign. Either of the changes

$$
f+f^{*} \text { and } g+g^{*} \text { or } f+-f^{*} \text { and } g+-g^{*}
$$

leaves both the differential cross section and the analyzing power unchanged, and it requires a measurement of the $A$ or $R$ parameter to resolve the ambiguity.

In the most common situation, only the differential cross section and the analyzing power are experimentally $m$ :asured. Under a linear transformation 25 


$$
\begin{aligned}
\left(\begin{array}{l}
f \\
g
\end{array}\right)+\left(\begin{array}{l}
F \\
G
\end{array}\right) & =e^{-i \theta \pi_{z}} e^{i \pi \sigma} y^{/ 4}\left(\begin{array}{l}
f \\
g
\end{array}\right) \\
& =\frac{1}{\sqrt{2}}\left(\begin{array}{c}
e^{-i \theta} f+e^{-i \theta} \\
-e^{i \theta} f+e^{i \theta}
\end{array}\right)
\end{aligned}
$$

and inverting this, we find

$$
\begin{aligned}
\left(\begin{array}{l}
f \\
g
\end{array}\right) & =e^{-i \pi \sigma_{y} / 4} e^{i \theta \sigma_{z}}\left(\begin{array}{l}
F \\
G
\end{array}\right) \\
& =\frac{1}{\sqrt{2}}\left(\begin{array}{l}
e^{i \theta} F-e^{-i \theta} G \\
e^{i \theta} F+e^{-i \theta} G
\end{array}\right) .
\end{aligned}
$$

Under this transformation the differential cross section becomes

$$
\begin{aligned}
I_{0} & =|f|^{2}+|g|^{2} \\
& =|F|^{2}+|G|^{2}
\end{aligned}
$$

and the analyzing power

$$
\begin{aligned}
I_{O} A_{N} & =2 \operatorname{Re}\left(\mathrm{fg}^{*}\right)=\mathrm{fg}^{*}+\mathrm{f}^{*} \mathrm{~g} \\
& =|\mathrm{F}|^{2}-|\mathrm{G}|^{2}
\end{aligned}
$$

So we can solve for $|F|$ and $|G|$, but the phase between them is completely undetermined.

The ambiguities in the amplitudes lead to corresponding ambiguities in the phase shifts. In $\pi^{+} p$ scattering if we assume $\ell \leqslant 1$ there are three amplitudes. Given a set of phase shifts $\delta_{\ell j}: \delta_{0,1 / 2}$, $\delta_{1,1 / 2}$, and $\delta_{1,3 / 2}$ there exists another set, from the Minami ambiguity, obtained from the former by

$$
\begin{aligned}
& \delta_{0,1 / 2} \nsucceq \delta_{1,1 / 2} \\
& \delta_{1,1 / 2} \nsucceq \delta_{2,1 / 2} \\
& \delta_{1,3,2} \rightleftarrows \delta_{2,3 / 2}
\end{aligned}
$$

such that the same differential cross section results. This is tecause the differential cross section depends on the total angular momentum $J$ 
but not on the parity (or angular momentum $\mathfrak{l}$ ). Allowing this ambiguity means that we have to allow $\ell$ up to 2 .

If we have a set of $\delta_{2 I, 2 J}$ that satisfy the observed differential cross section, then there exists, from the Yang ambiguity, a second set $\delta_{2 \mathrm{I}, 2 \mathrm{~J}}$ related to the first by

$$
\begin{aligned}
\delta_{3}^{-} & =\delta_{3} \\
\delta_{33}^{-}-\delta_{31}^{-} & =\delta_{31}-\delta_{33}
\end{aligned}
$$

such that $\left|E^{-}\right|^{2}=|f|^{2}$ and $\left|g^{-}\right|^{2}=|g|^{2}$. These two equations leave one common undetermined constant in the phase shifts $\delta_{31}$ and $\delta_{33}$. This ambiguity is particularly interesting because when the phase shift $\delta_{33}$ is equal to $\pi / 2$ as is the case in the neighborhood of the $\Delta_{33}$ resonance, the phase shift $\delta_{33}^{-}$(and $\delta_{3 l}$ ) will in general, not even be in the neighborhood of $\pi / 2$. This shows that a resonance in one channel can be caused by the combined effect of several phase shifts, none of which passes through $\pi / 2$.

From eq. (1.137) we see that $\delta_{33}-\delta_{31}$ can change sign. The phase shifts $\delta_{33}$ and $\delta_{31}$ themselves also change, and we see that the polarization of the recoiling proton is different for the two sets of phase shifts (see eq. (1.133)). Consequently, we can decide which of these sets is the correct one by measuring the polarization of the recolling proton (or equivalently the analyzing power). 
Finally, as long as the Coulomb term is neglected, the cross section is invariant under a change of sign of all phase shif:s involved (i.e. $f+-f, g+-g)$. This ambiguity can be removed ${ }^{26}$ by looking at the interference between $\pi^{+}$nuclear and Coulomb scattering amplitudes (which we know to be repulsive). When $\delta_{3} 3$ is positive one expects a destructive interference, indicating that the dominant part of the nuclear force is attractive. This destructive interference with the Coulomb term causes a sharp dip in the differential crose section at small angles. When $\tilde{\jmath}_{33}$ is negative, the interference is constructive and the diffeiential cross section has a smooth behavior. 
CHA.PTER TWO

THE EXPERIMENT

\subsection{The Pion Beam}

The experiment was performed at the Los Alamos Meson Physics Facility (LAMPF) in the east cave of the Pion Particle Physics $\left(P^{3}\right)$ channel. This channel provides positive or negative pion beams over a broad range of momenta, $190 \mathrm{MeV} / \mathrm{c}<\mathrm{p}<730 \mathrm{MeV} / \mathrm{c}$, with a momentum bite $0.0025<\Delta \mathrm{P} / \mathrm{F}<0.1 .27$ LAMPF is a high-intensity, linear proton accelerator which is more than half a mile long. By using high-purity hydrogen gas in special ion sources, three beams are available at LAMPF : $H^{\dot{r}}$ (hydrogen nuclei, or protons), $\mathrm{H}^{-}$(hydrogen atoms with an extra electron), and $\mathrm{P}^{-}$(polarized $\mathrm{H}^{-}$beam). The facility utilizes a three-stage linear accelerator (linac) that can simultaneously accelerate the $\mathrm{H}^{+}$beam and either the $\mathrm{H}^{-}$or $\mathrm{P}^{-}$beam. The first stage is an injector which accelerates $\mathrm{H}^{+}$and either $\mathrm{H}^{-}$or $\mathrm{P}^{-}$ions from their respective sources to $750 \mathrm{KeV}$. At this stage the ion source is pulsed at $120 \mathrm{~Hz}$ rate, while each beam pulse is 570 micro-seconds long, giving a duty factor of $6.84 \%$ for the accelerator (i.e. beam is on only for 


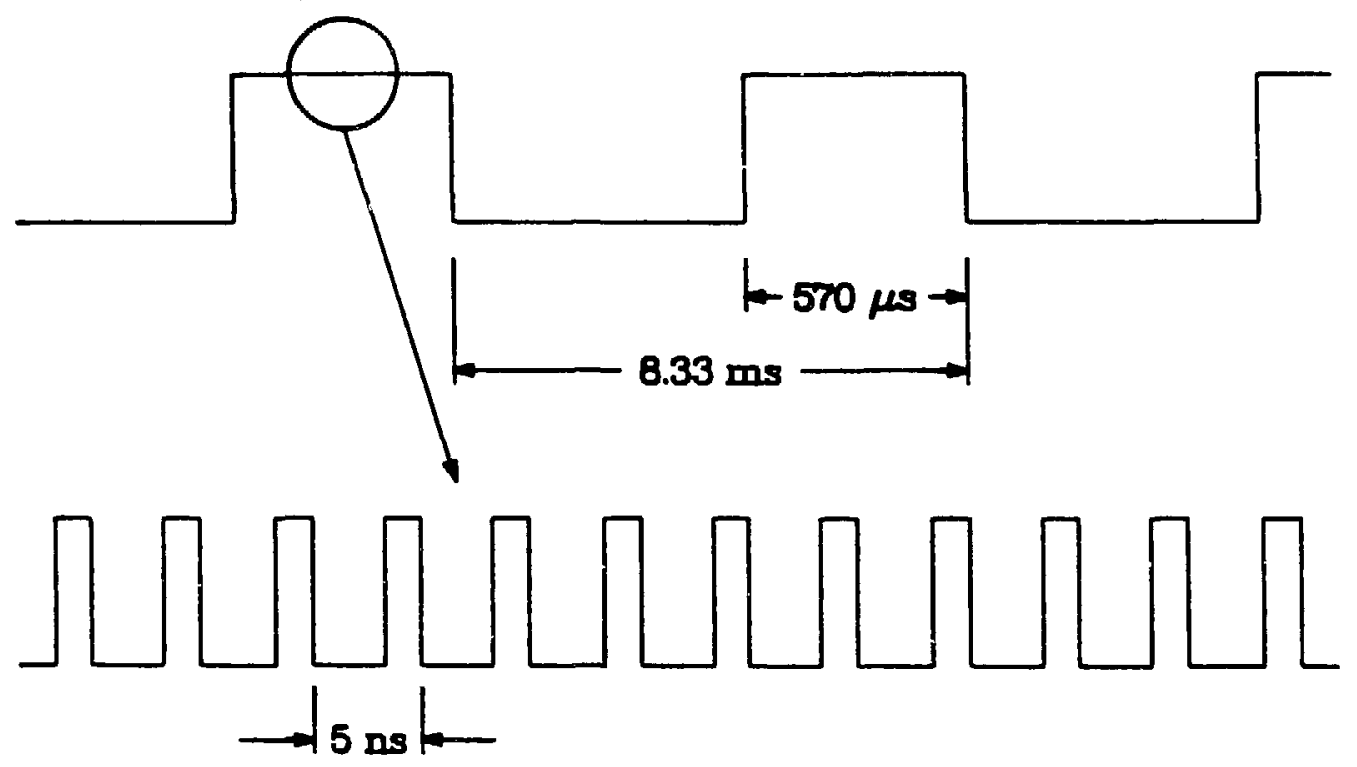

Figure 2.1. Time structure of the LAMPE $\mathrm{H}^{+}$beam. 
$570 \mu$ s every $8.33 \mathrm{~ms}$ ). This is the beam macrostructure. Higher duty factors are possible, and are achieved by lengthening the macropulse. In the second stage an Alvarez type drift tube (or standing wave section) operating at $201.25 \mathrm{MHz}$, accepts ions from two of the three injectors, accelerates the ions to $100 \mathrm{MeV}$, and sends them to the main accelerator. This gives a microstructure to the beam; each of the 570 us long macro puises consists of "micro buckets", every $1 / 201.25 \mathrm{MHz}=4.97 \mathrm{~ns}$ or approximately every $5 \mathrm{~ns}$. This is the $201 \mathrm{MHz}$ pulsed time structure of the beam. Figure 2.1 shows the time structure of the $\mathrm{H}^{+}$beam. In the third and firal section of the accelerator, the particles are accelerated up to $800 \mathrm{MeV}$, using the side-coupled cavity waveguide linac developed at Los Alamos. ${ }^{28}$ The frequency of this section of the linac, $805 \mathrm{MHz}$ is chosen to be the fourth harmonic of the second stage. The final proton beam energy is stepwise variable from $100 \mathrm{MeV}$ to $800 \mathrm{MeV}$. Figure 2.2 shows the accelerator. The $\mathrm{H}^{+}$and $\mathrm{H}^{-}$(or $\mathrm{P}^{-}$) beams enter the beam switchyard area shown in figure 2.3 , where they ara magnetically separated. In the switchyard the beams are directed to the four main beam lines. Line A gets most of the $\mathrm{H}^{+}$beam. There are four production targets in line A: a thin target used for nuclear-chemistry studies; the A-1 target, $3 \mathrm{~cm}$ carbon, provides the pion beam (and other particles) for the Low Energy Pion (LEP) channel and the Energetic Pion Channel and Spectrometer (EPICS); the A-2 target, $5 \mathrm{~cm}$ carbon, provides the pion beam for the Pion Particle Physics $\left(\mathrm{P}^{3}\right)$ channel and a muon bean for the Stopped Muon Channel (SMC); the A-5 target, $8 \mathrm{~cm}$ carbon, which provides a pion bean for the biomedical experimental area. The A1 and A2 


\section{CLINTON P. ANDERson}

mESON PHYSICS FACILITY

\section{EXPE RIWEFTAL AAEA C
HIGH RE SOLIITHOM PAOTON SPECTMOETETE}

"IS

15

ENG - A/LAMPF
OFFICES

COOL ING TOWEAS

MUCLEAR CHEMISTRY LABORATORY 417

ENTRance 5 TATION

WATER

STORAGE

,

Nin

لv"

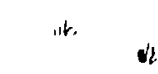

l.

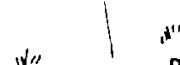

D.

WAREHOUSE

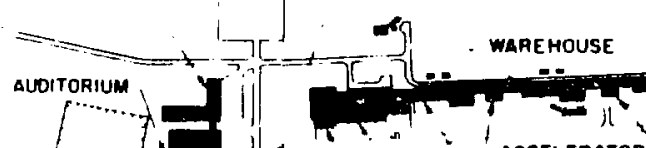

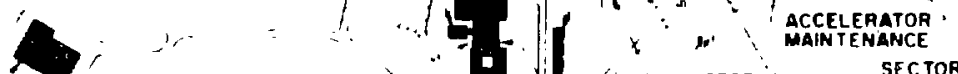

VISITOR CENTER $* " 11, \%$ SEctón a

14 FMIT LaBoratory gutDING EOUIPMENT TEST
LABOFATORY

"1

$$
\text { "Laboratory-OFFICE }
$$

"12

AT-I
OFFICES

i)

ACC. TECHWOLOGY LABORTORY
LAMPF OPERATIONS BUILOING

$\rightarrow$ Ho

BUILDING

แ3

$\frac{200}{0.0200 \quad 400 \quad 000}$

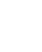

$$
\begin{aligned}
& \text { ACCELERATOR } \\
& \text { TET.HNOI ONIY } \\
& \text { OF YICE } \\
& \text { BUIIUNCS }
\end{aligned}
$$

no

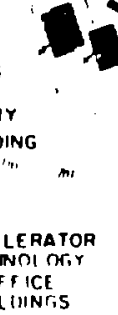

WNR CONTAOL
BUILDING
WNR OFFICE
RIJLTINTSS

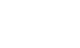

" WAREHOUSE

AND THERAPY

"11

EXPE RIMENTAL AREA B

EXPERIMENTAL AREA A 10

RADIATION DAMAGE AND

ISOTOPE PaOduction

NEUTAINO RESEAACH

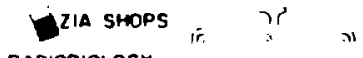

RADHOBIOLOGY

SEWAGE LAGOOHS

EXPERIMENTAL AREA SMMPS

BEAM SWITCHYAHO IT

WNA SUPPORT 


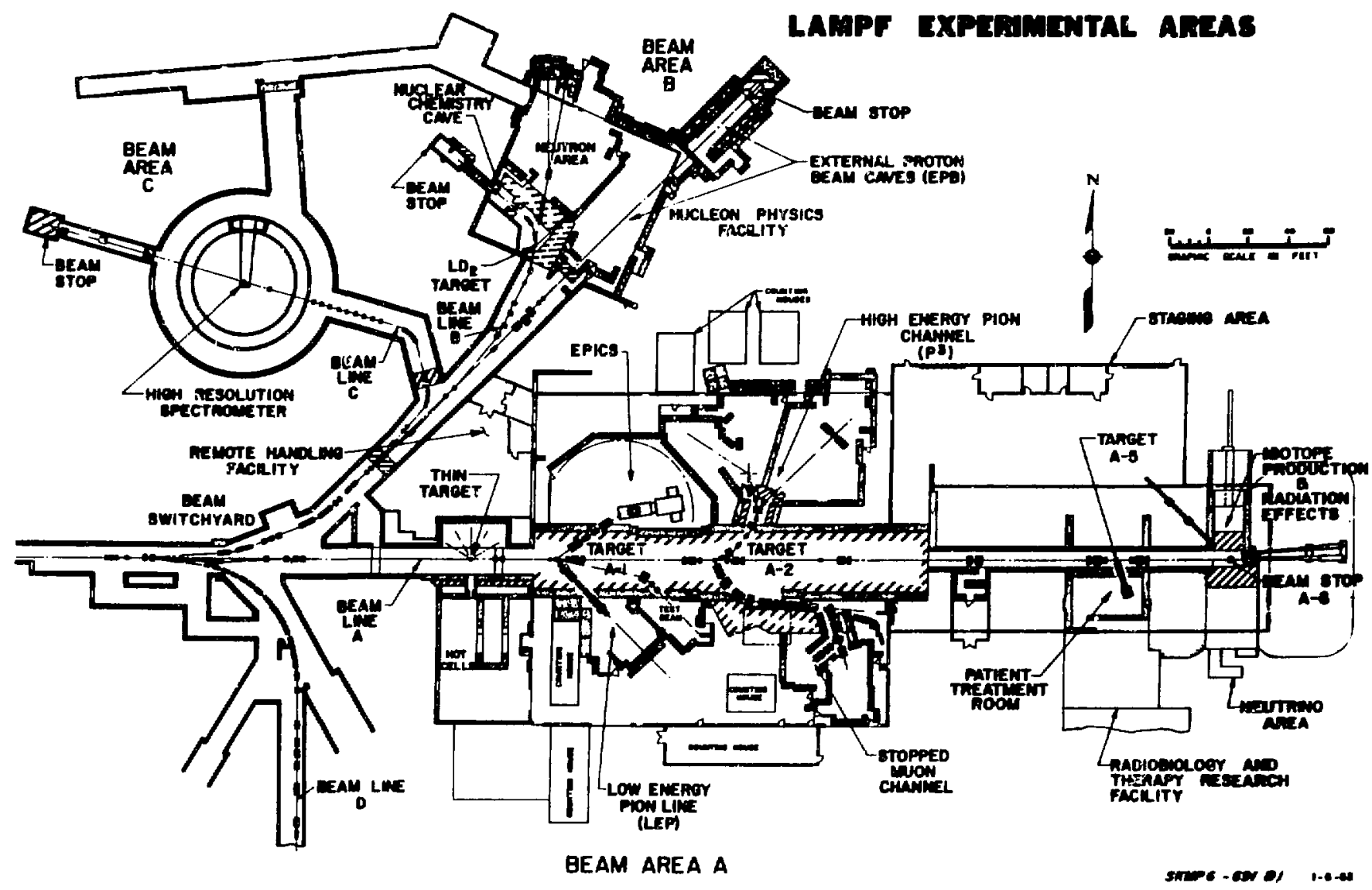

Figure 2.3. Beam switchyard and experimental area A. 
targets are continuously in the beam. The experimental area A which contains several secondary beam lines, including the $\mathrm{P}^{3}$ channel, is also shown in figure 2.3. A falrly detailed description of LAMPF and its facilities is given in the LAMPF users Handbook. ${ }^{29}$ To transport the pirns produced in the A-2 production target by the main proton beam to the $\mathrm{P}^{3}$ experimental area, three rectangula: bending magnets (dipoles) are used. The bend planes are horizontal. Each bending magnet has two entrance and two exit quadrupole magnets. This three-bend analyzing system selects and momentum analyzes positive or negative pions. The production angle is $20^{\circ}$. After the first bend which determines the momentum and polarity of the pions, the beam is focused between QM-04 and QM-05 where a set of slits, MS03, define the channe1's momentum acceptance. A degrader may be inserted to provide chromatic separation of $\pi^{+}$and $P$ which initially had the same momentum. BM02 translates this $L$ romatic separation into spatial separation at the position of the absorber slits. A second focus occurrs in the center of the last bending magnet (BM-03). This magnet steers the beam to the east or west cave. Horizontal and vertical slits near the A-2 production target are used to control the pion beam intensity. A diagram of the $\mathrm{P}^{3}$ channel is shown in figure 2.4. Extensive studies of the $\mathrm{p}^{3}$ beam line were conducted by our group previously. 30

The polarized proton target was located 6 meters from the last quadrupole in the channel (QM-16). The channel was tuned using the results of a computer simulation for the 6 meter focus. A beam spot size of $2 \mathrm{~cm}$ by $2 \mathrm{~cm}$ at the target position was specified as input to a 


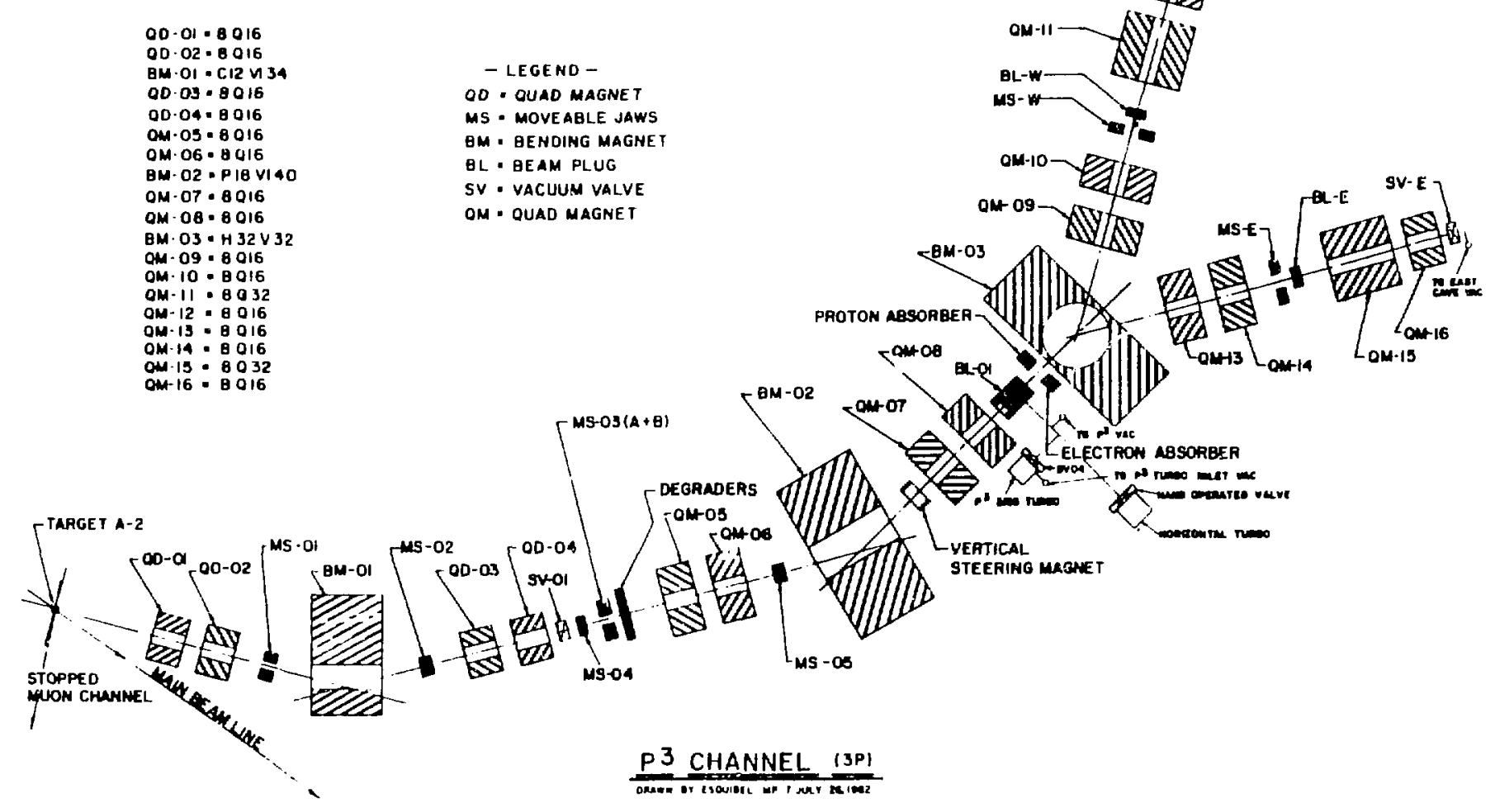

Figure 2.4. The Pion Particle Physics $\left(p^{3}\right)$ Channel. 
TRANSPORT ${ }^{31}$ program. TRANSPORT runs for a beam momentum of $350 \mathrm{MeV} / \mathrm{c}$. The resulting magnetic field outputs were input to a second program, SHUNT, which produced the corresponding shunt voltages for the beam line magnets (SHUNT scales the momentum). Fine tuning was accomplished with the aid of two Beam Profile Monitors, BPMkl ( 3.0 meters upstream of the target) and BPM非 ( 1 meter upstream of the target) shown in figure 2.5. The last two bending magnets (BM-02 and BM-03) were alternately fine tuned to bring the heam on the beam line. The last two quadrupole magnets (QM-15 and $Q M-16)$ were also adjusted so that the beam was brougti to a waist at the target position. Shunt readings of each magnet in the beam line are listed in appendix A. Two more steering magnets at the end of the $\mathrm{P}^{3}$ channel were used to compensate for the bending of the beam in the field of the polarized target magnet and to correct for mis-steering of the beam. The $x$-steerer was used to steer the beam in the horizontal plane and the y-steerer, which was usually off during the course of the experiment, steered the beam vertically. These steering magnets are also shown in figure 2.5. A summary of the beam characteristics are given in table 2.1. Bean intensity was obtained from visual scalers, and the bending due to the polarized target magnetic field was calculated for the beam central ray. The bending of the particles in this ragneric field causes an of iset in the scattering angle and in the target vertex. 


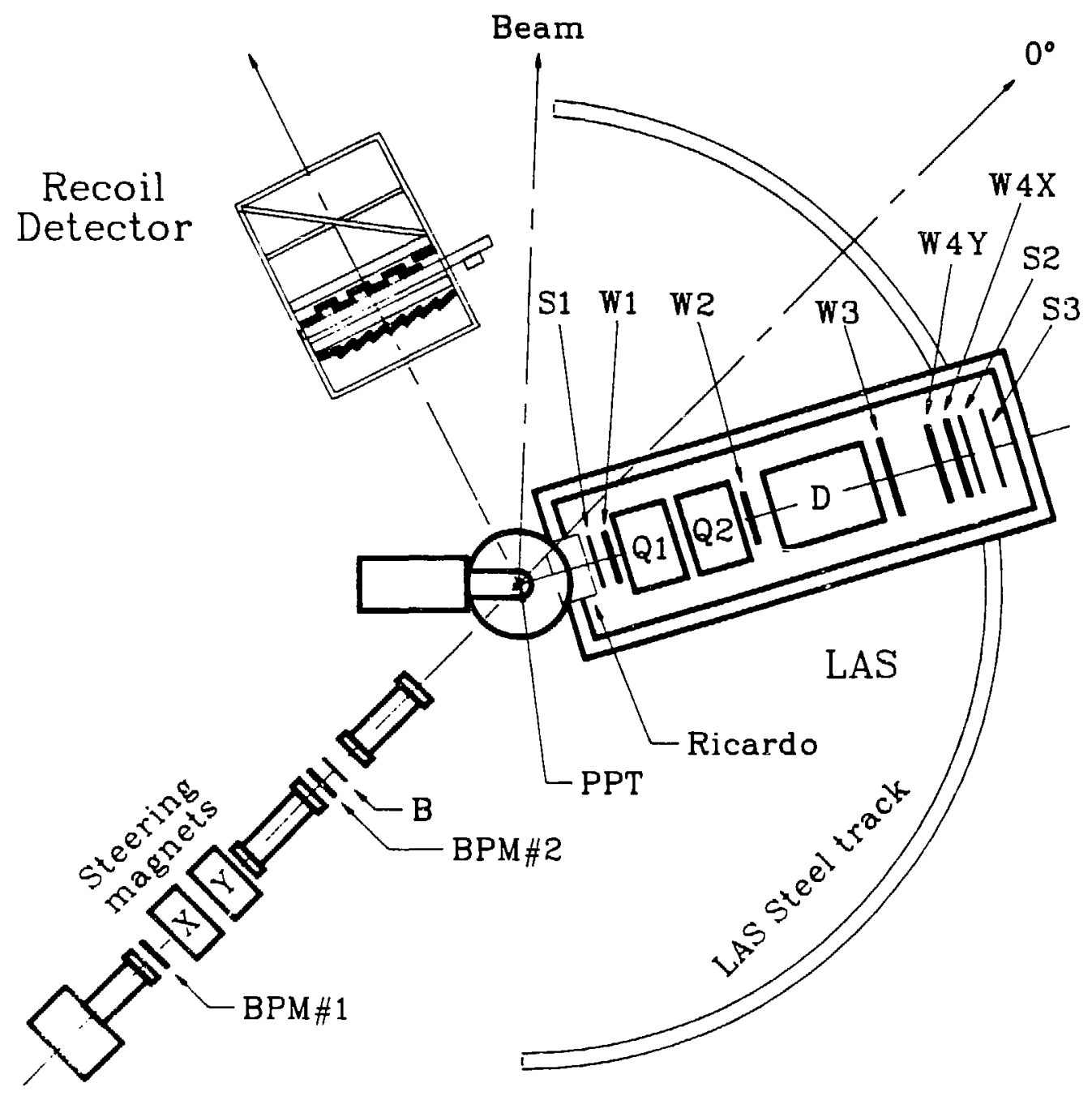

Figure 2.5. Layout of the experimental setup. 
Table 2.1. Sumnary of beam characteristics.

\begin{tabular}{|c|c|c|c|c|}
\hline $\begin{array}{l}\text { Beam Momentum } \\
\text { at center of target } \\
(\mathrm{MeV} / \mathrm{c})\end{array}$ & $\begin{array}{l}\text { Polarity } \\
\pi^{+} \text {or } \pi^{-}\end{array}$ & $\begin{array}{c}\text { Rate } \\
\text { (pions/sec) } \\
\left(\times 10^{5}\right)\end{array}$ & $\begin{array}{l}\text { FWHM } \\
\Delta \mathrm{p} / \mathrm{p} \\
(\%)\end{array}$ & $\begin{array}{l}\text { off set } \\
\text { (Degrees) }\end{array}$ \\
\hline 470.85 & $\pi^{+}$ & $0.38-3.10$ & 2.0 & 18.1 \\
\hline$"$ & $\pi^{-}$ & $0.41-0.56$ & $"$ & $"$ \\
\hline 546.86 & $\pi^{+}$ & $0.091-2.99$ & 1.3 & 15.5 \\
\hline$"$ & $\pi^{-}$ & $0.20-0.44$ & $"$ & $"$ \\
\hline 624.86 & $\pi^{+}$ & $0.020-2.70$ & 1.6 & 13.5 \\
\hline$"$ & $\pi^{-}$ & $1.90-3.60$ & $"$ & $"$ \\
\hline 687.00 & $\pi^{+}$ & $0.078-1.50$ & 5.3 & 12.3 \\
\hline$"$ & $\pi^{-}$ & $0.20-0.40$ & $"$ & $"$ \\
\hline
\end{tabular}




\section{1 .1 Beam Monitoring}

The beam spot was monitored using two beam profile monitors upstream of the target and a hole counter at the target position. The beam spot size at the target was approximately $2 \mathrm{~cm}$ in diameter as measured with polaroid film. The LAMPF beam profile monitor ${ }^{32}$ consisted of a pair of multiwire proportional chambers, each with a xand $y$-plane. The two planes were separated by $1 \mathrm{~cm}$, and each contained 64 wires with $2 \mathrm{~mm}$ wire spacing. The chambers were filled with argon and operated at about $-2.4 \mathrm{kV}$. An input amplifier converted the current from each wire to a voltage. A peak detector and a capacitor latched the output voltage's peak value, enabling a muliplexer to scan all 64 wires. The output from each chamber was displayed on an oscilloscope, providing a histogram of the voltage for each wire. This arrangement was most convenient for beam tuning and provided assurance that the beam was on target. The profile monitors stayed in the beam throughout the experiment, and were also used to verify that beam line components had not drifted during data-taking.

The high pion flux prevented the use of absolute beam monitors, nor were absolute rates needed in this experiment. Relative beam intensities were measured using two similar plastic scintillation counter te:escopes above and below the beam line at the same distance from the central line. The telescopes were sensitive to muons from pion decay in-flight and to pions in the beam scattered at small angles by the windows and beam monitors. Also a scintillation counter 
directly in the $b-\pi$ was used; it sagged at high beam rates. Each of the two telescopes consisted of two counters, M1 and M2. M1 was $4.5 \mathrm{~cm}$ by $4.5 \mathrm{~cm}$ and $0.64 \mathrm{~cm}$ thick, M2 was $10.2 \mathrm{~cm}$ by $10.2 \mathrm{~cm}$ and $0.64 \mathrm{~cm}$ thick. M2 was $8 \mathrm{~cm}$ behind $M 1 . M 1 U M 2 U+M 1 D \cdot M 2 D$ was used as the relative beam monitor. 


\subsection{The Target}

A polarized proton target (PPT) with the axis of polarization perpendicular to the scattering plane was used. The polarized proton target system consisted of eight sub-systems. The inter-relation of these sub- rstems will be discussed briefly in sections 2.2 .1 through 2.2.8. Figure 2.6 shows the elements of the polarized taiget. A more detailed discussion may be found in the thesis of Tippens. ${ }^{3}$ See also the polarized proton target handbook. 34

In order to polarize protons a magnetic field $\vec{B}$ splits the two energy levels:

$$
\begin{aligned}
& E_{+}=-\mu_{P} B \text { for proton spin parallel to } \vec{B} ; \\
& E_{-}=+\mu_{P} B \text { for proton spin anti-parallel to } \vec{B},
\end{aligned}
$$

where $\mu_{\mathrm{F}}=8.8043 \times 10^{-18} \mathrm{MeV}$ Gauss ${ }^{-1}$ is the magnetic moment of the proton. The two energy levels are then populated according to Boltzman statistics 35 in the ratio

$$
\frac{\langle t\rangle}{\langle-\rangle}=e^{\frac{2 \mu_{P^{B}}}{K_{B}^{T}}}
$$

The "+" represents a spin parallel to the field state, and "-" a state of spins anti-parallel to the field. To get a high polarization $a$ strong field $B$ and a low temperature $T$ is needed. Typical values are $\mathrm{B}=25 \mathrm{KG}$ and $\mathrm{T}=0.5^{\circ} \mathrm{K}$, so that

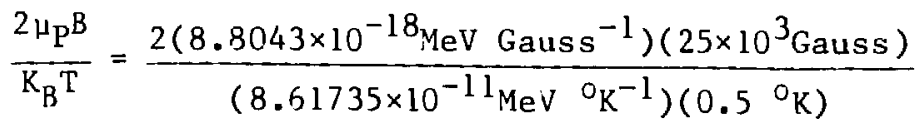

$$
\begin{aligned}
& =\mathrm{i} .0217 \times 10^{-2} \text {, }
\end{aligned}
$$




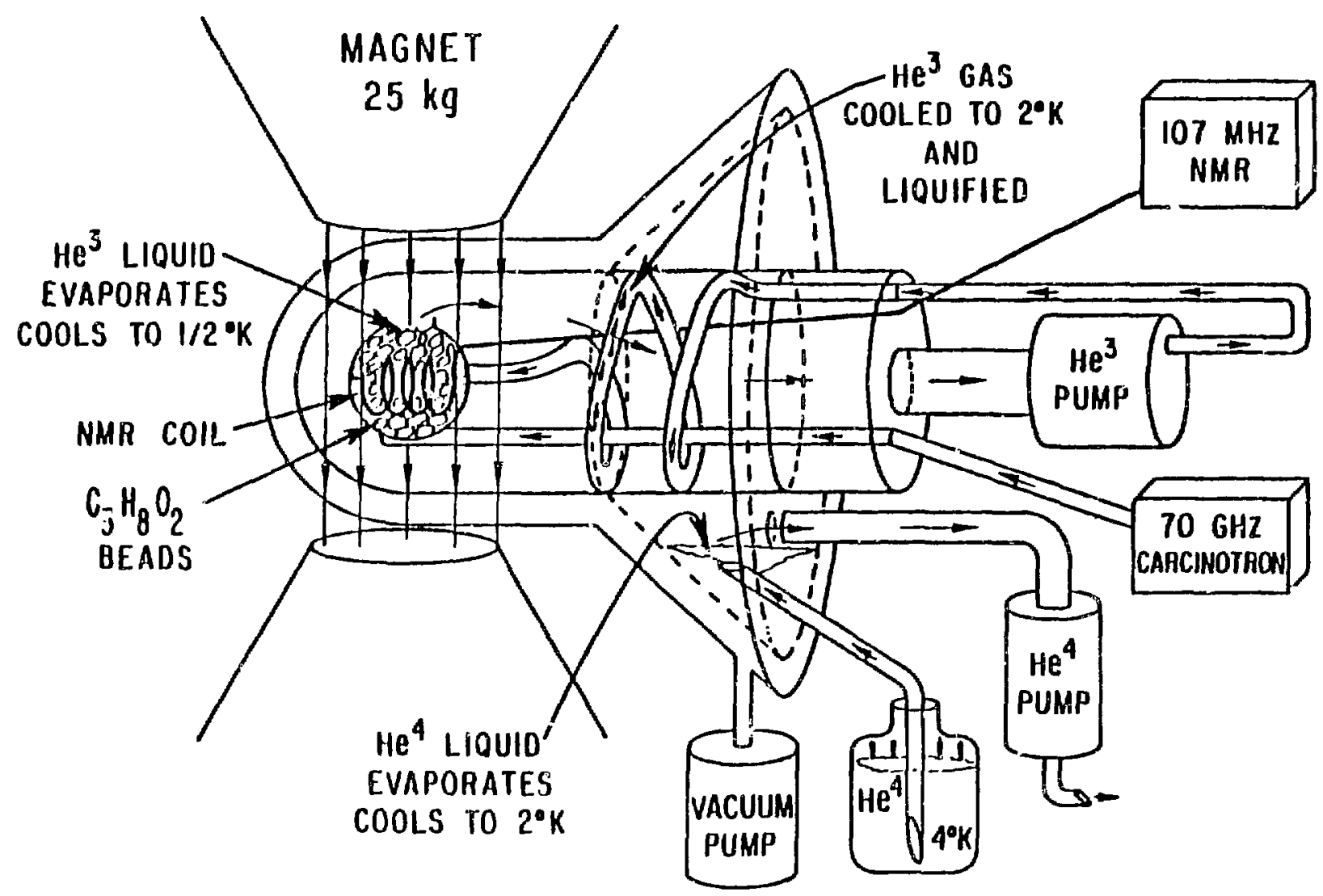

Figure 2.6. Elements of the polarized target (PPT). 
thus

$$
\frac{\langle t\rangle}{\langle-\rangle}=1.01
$$

This means that states with proton spin parallel to the magnetic field are almost as populated as the states with proton spin antiparallel to the magnetic fleld; this corresponds to the polarization

$$
\frac{\langle+\rangle-\langle-\rangle}{\langle+\rangle+\langle-\rangle}=0.0051
$$

i.e., under these conditions the thermal equilibrium polarization is onl $30.51 \%$. This is used for absolute calibration but is not useful as a polarized target. For electrons, however, having a much larger wagnetic dipole moment $\mu_{\mathrm{e}}=0.5795 \times 10^{-14} \mathrm{MeV}^{-10 u s s}{ }^{-1}$ we find under the same conditions that

$$
\frac{\langle+\rangle-\langle-\rangle}{\langle+\rangle+\langle-\rangle}=.9976
$$

i.e. the electrons are almost $100 \%$ polarized. The target polarization is enhanced with the Dynamic Nuclear Polarization (DNP) technique using microwaves to transfer the polarization from the electrons to the protons. 36 Operationally this technique can be described as follows: a diamagnetic insulator containing a small concentration of fixed paramagnetic impurities is bombarded with mirowaves on either side of the Eleccron Spin Resonance (ESR) curve at a frequency $\nu=\nu \mathrm{e}^{+\delta}$ where $|\delta| \ll\left|v_{e}\right|$. This can lead to an enhanced nuclear polarization, comparable to the electronic polarization. 36,37

$$
\begin{aligned}
& \text { Application of microwaves of enerby } \\
& \qquad h \nu=2 \mu_{e^{B}}+2 \mu_{p} B
\end{aligned}
$$


which correspond to the frequency

$$
v=70.06 \mathrm{GHz} \pm 106.4 \mathrm{6Hz}
$$

simultaneously flips an electron and a proton spin. The alectron quickly realigns ready to flip another proton. The proton, however, can not flip back unless it interacts with the atomic lattice, so it stays put typically for several hours. Thus by folarizing the electrons, the protons are then polarized by transferring the electron polarization to the protons using DNP technique.

Consider the energy levels of an isolated electron-proton pair of spins $S=1 / 2, I=1 / 2$ in a strong magnetic field. In the absence of dipole-dipole interaction between $S$ and $I$ the four energy states: $\left|a_{0}\right\rangle=|++\rangle,\left|b_{0}\right\rangle=|+-\rangle . \quad\left|c_{0}\right\rangle=|-+\rangle$, and $\left|d_{0}\right\rangle=|--\rangle$ are pure, where the $\left|s_{z}(e){ }_{z}(p)\right\rangle$ refer tc the $z$-component of the electron and proton spin respectively. Double spin flip transitions by an $R F$ field are forbidden since they involve changes in angular momentum of $\Delta J=0$ for $|+\rangle+|+\rangle$ transitions and $\Delta \mathrm{J}=2$ for $|++\rightarrow+|-\rangle$ transitions, while the RF field can only induce $\Delta J=1$ transitions.

The dipole-dipole interaction between $\vec{\mu}_{\mathrm{S}}=\gamma_{\mathrm{S}} \stackrel{\overrightarrow{\mathrm{S}}}{\text { and }} \vec{\mu}_{\mathrm{I}}=\gamma_{\mathrm{I}} \overrightarrow{\mathrm{I}}$

$$
\frac{\gamma_{I} \gamma_{S}}{r^{3}}[\vec{I}+\vec{S}-3(\vec{I} \circ \vec{r})(\vec{S} \cdot \hat{r})]
$$

(where $r \equiv|\vec{r}|$ is the distance between the two spins and $\hat{r}=\frac{\vec{r}}{r}$ ) scraubles chese four pure states, making them into the perturbed states $|a\rangle,|b\rangle$, $|c\rangle,|d\rangle$ represented in figure 2.7 . The coefficients $F$ and $q$ may be calculated by perturbation theory. Since for electrons the magnetic 

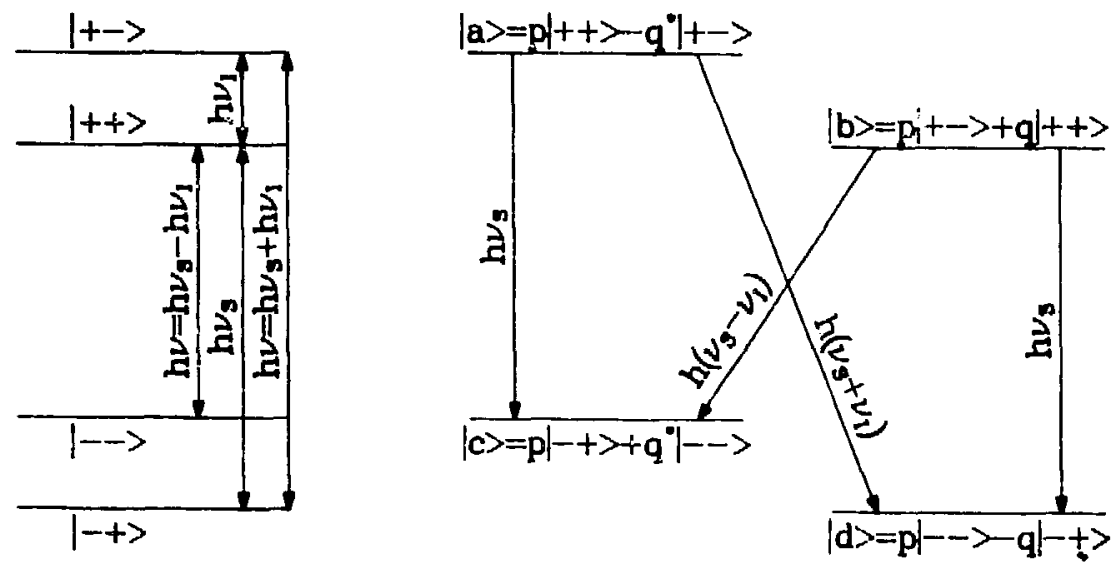

Figure 2.7 (a) Energy levels of an isolated electron-proton pair in a strong magnetic field. (b) Eigenstates of an electron-proton pair coupled by dipole-dipole interactions in a strong magnetic field.

moment $\vec{\mu}$ and spin $\vec{S}$ are antiparallel the lower energy states are those with electron spin antiparallel to $\vec{B}$.

Assume that the electrons which have spins $S$ are completely polarlzed, say all down, and the nucleons with spin I are completely unoolarized. The dipole-dipole interaction permits the simultaneous reversal of $\mathrm{S}$ and $\mathrm{I}$ spins in opposite directions, and also the reversal in the same direction. In such reversal the total energy of the spin system changes by an amount $h\left(v_{S} \pm v_{I}\right)$ and the process will not occur unless the missing energy is supplied by the crystalline latice. Suppose now that an external source of microwave energy of irequency $v=v_{S} \pm v_{I}$ induces either $|+\rangle++|-+\rangle$ or $|+\rangle \rightarrow|-\rangle$ transitions. Assume also that the electron line width $\Delta \nu_{S}$ is much smaller than the nuclear frequency $v_{I}$ so that when $|+\rangle++|-+\rangle$ transitions are induced $\left(v=v_{S}-v_{I}\right)$, the $|H \rightarrow| \rightarrow$ transitions $\left(v=v_{S}+v_{I}\right)$ can not occur because they are off-resonance with the driving frequency $v$, and vice versa. Assume that we drive, say, forced $|+\rangle+\rightarrow|+\rangle$ transitions and that the 
strength of the source is such that the rate at which they occur is much greater than the nuclear relaxation rate $\left(1 / T_{n} \simeq 10^{-3} \mathrm{~s}^{-1}\right.$ is typical for polarized target material). The reversal of an electron spin alone, caused by its coupling to the lattice, occurs at a much faster rate $\left(1 / \mathrm{T}_{\mathrm{e}} \approx 10^{3} \mathrm{~s}^{-1}\right)$.

Consider first a spin I that is down. All the S spins being down, the spin I could only do a $|-\rightarrow+|++\rangle$ transition, which is forbidden as it is off-resonance. On the other hand, a spin I that is up may do a $|-+\rangle+|+-\rangle$ transition with a spin $S$ that is down, ending in a situation where $I$ is down and $S$ is up. This spin $S$, which is now up, is a danger to all of the I spins that are down, since it could bring one of them up through a forced $|+\rangle+|-+\rangle$ transition if it were not for the fast electron-lattice relaxation rate which flips the up spin $S$ down without affecting the spin $I$. Tf the rate at which $|-+\rangle+\mid+->$ transitions are induced (determined by the microwave power) is slower than the electron-lattice relaxation rate the cycle can start again until all the I spins are down (parallel to electron spins). Then the high electron polarization will be maintained and one can ideally polarize the I spins via the $S$ spin polarization. If the source frequency $\quad v=v_{S}+v_{I}$ drives $|--|+|+\rangle$ rather than $|-+\rangle+|+-\rangle$ transitions, the I spins will go lip with a polarization opposite to that of the $S$ spins. For an electron polarization $\left|P_{e}\right|<1$, these processes lead to a nuclear polarization $P_{n}= \pm P_{e}$. 


\subsubsection{The Polarized Proton Target Magnet (PTM)}

The magnet is a conventional iron-core, water-cooled, C-shape 22-inch Varian magnet with a $7.62 \mathrm{~cm}$ pole-tip gap which produces a uniform field of $25 \mathrm{KG}$ over the target volume. The beam passed through a cylindrical hole $13 \mathrm{~cm}$ in diameter at the back of the yoke so that an angular range of $88^{\circ}$ to $-155^{\circ}$ was accessible. Since small nonuniformities of the PTM fiold within the target reduce the maximum polarization attainable, the target volume was 1 imited to $12.57 \mathrm{~cm}^{3}$, with a \pm 5 Gauss maximum variation of the field.

At the early stages of the experiment it was realized that the PTM would reduce acceptance of the spectrometer at low momenta. So to increase the acceptance a horizontal steering magnet of the WERBECKA type was mounted at the spectrometer entrance. Because of the proximity of this steering magnet ("Ricardo" in figure 2.5) to the PTM, a possible distortion of the PTM central field was anticipated. However, detailed maps of the combined PTM-Ricardo field showed that (see figure 2.8) the uniformity of the PTM central fleld was uraffected to a few parts in $10^{5}$. Plots of the PTM field as a function of the radial distance from the center of pole tip gap in the horizcntal plane midway between the pole tips is shown in figure 2.9 .

\section{$2.2 .2{ }^{4}$ He System}

The ${ }^{4}$ He system provide a bath of liquid ${ }^{4} \mathrm{He}$ (the evaporator) at a temperature of about $2{ }^{\circ} \mathrm{K}$ to liquify ${ }^{3} \mathrm{He}$. Liquid ${ }^{4} \mathrm{He}$ at a temperacure of $4{ }^{\circ} \mathrm{K}$ is drawn froni a dewar into the cryostat. The ${ }^{4}$ He 1iquid passes 


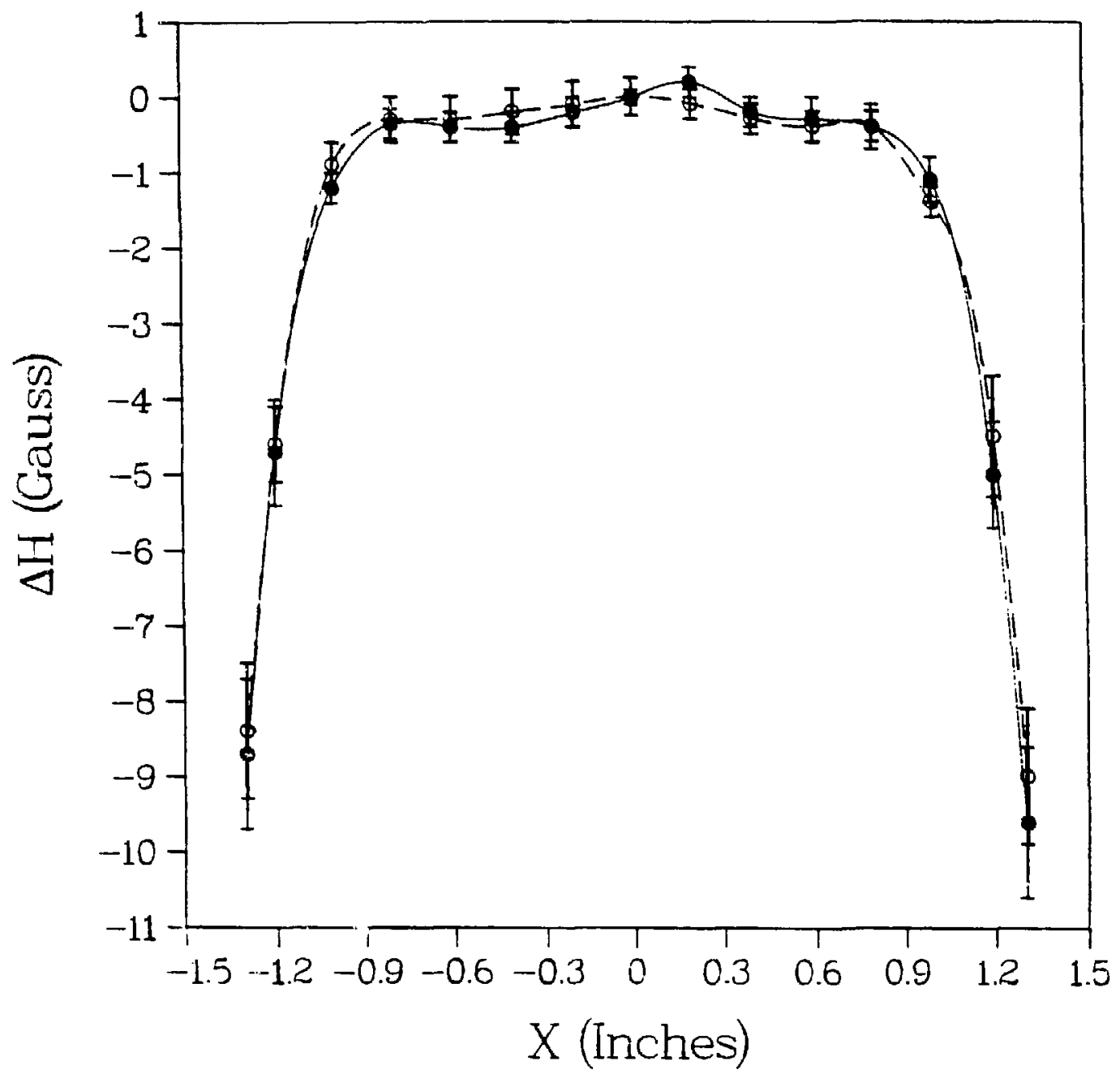

Figure 2.8. PTM-Ricardo field maps.

(•) Ricardo in place.

(o) Ricardo removed. 


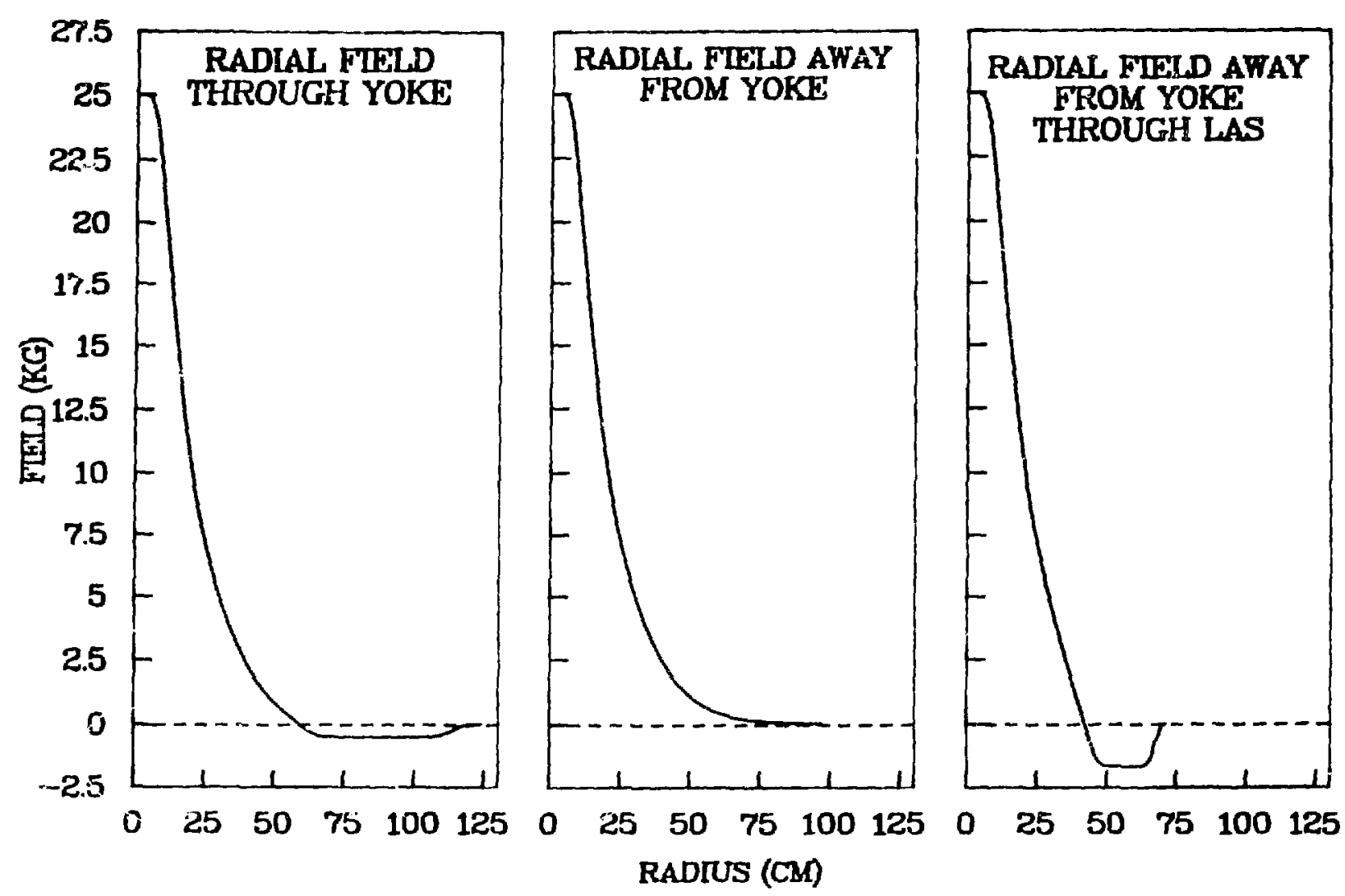

Figure 2.9. Field maps of polarized target magnet (PTM). 
through a filter before entering the cryostat. This filter reduces the temperature instabilities in the target caused by water or alr-1ce blocking the tiny valves in the cryostat. The ${ }^{4}$ He liquid flows through the separator valve into the separator (see figure 2.10) whose main function is to separate the gas that was bolled off in transferring it from the dewar to the cryoscat and provide a reservoir of liquid to feed the evaporator. The separated gas is used to cool the heat shields and the first ${ }^{3} \mathrm{He}-{ }^{4} \mathrm{He}$ heat exchanger before leaving the cryostar. Liquid ${ }^{4}$ He flows frum the separator through the evaporator valve into the evaporator tank which runs at $2{ }^{\circ} \mathrm{K}$. Evaporation of ${ }^{4} \mathrm{He}$ In the evaporator liquifies ${ }^{3}$ He gas in the liquifier since they are in close thermal contact. Gas from the evaporacor then flows through the second ${ }^{3} \mathrm{He}^{4} \mathrm{He}$ heat exchanger which cools the incoming ${ }^{3} \mathrm{He}$ gas to a

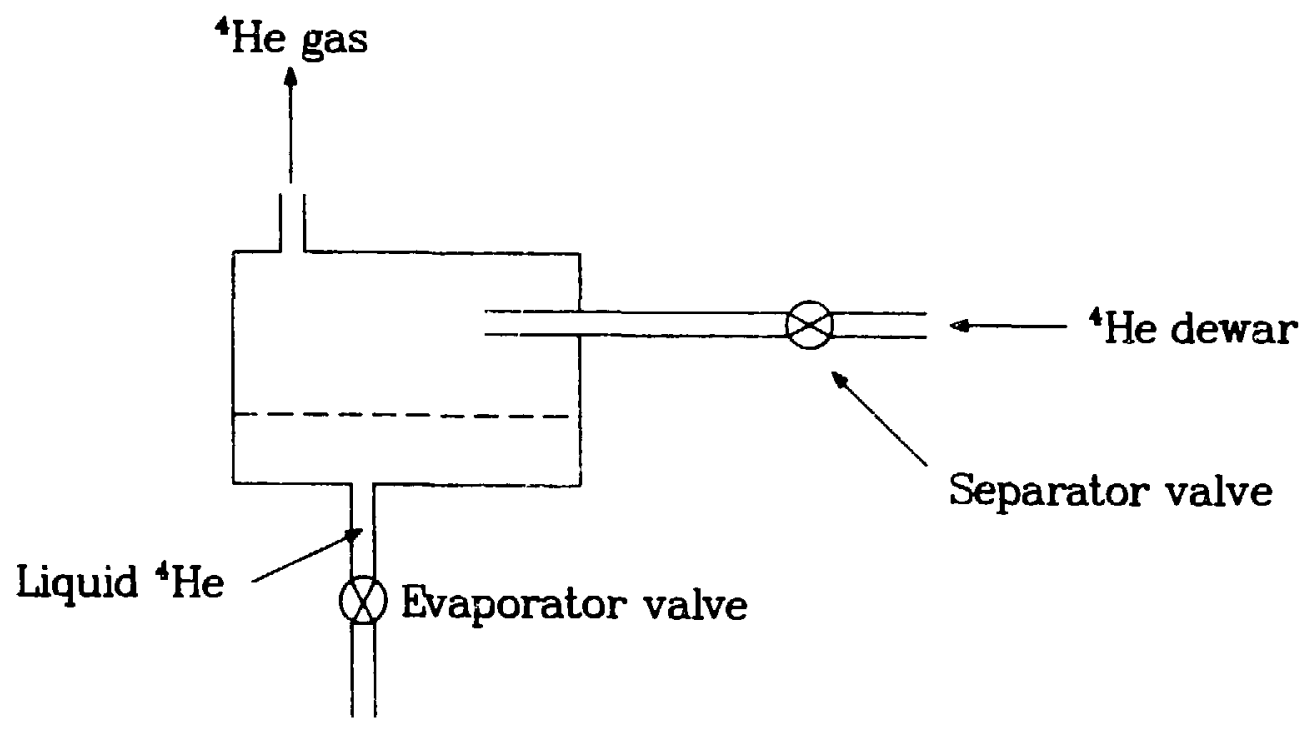

Figure 2.10. The separator. 
temperature just above the liquification point before leaving the cryostat.

\section{$2.2 .3^{3} \mathrm{He}$ System}

The ${ }^{3}$ He system provide a bath of 1 iquid ${ }^{3}$ He at a temperature below $0.5{ }^{0} \mathrm{~K}$ to cool the target material. ${ }^{3} \mathrm{He}$ is expensive so it flows in a closed loop. The ${ }^{4}$ He and ${ }^{3}$ He systems are physically separate but in thermal contact in the target cryostat. ${ }^{3} \mathrm{He}$ is a more desirable refrigerant than ${ }^{4} \mathrm{He}$ in the $0.5-1{ }^{\circ} \mathrm{K}$ region because it does not become a superfluid at these temperatures and control of the liquid flow is accomplished with conventional needle valves. Secondly, its vapor pressure below $2{ }^{\circ} \mathrm{K}$ is much higher allowing a much larger heat transfer. Figure 2.11 shows the ${ }^{3}$ He vapor pressure vs temperature below $1 \mathrm{o}_{\mathrm{k}} .38{ }^{3} \mathrm{He}$ gas enters the cryostat at a pressire somewhat less than an atmosphere. It is first precooled by thermal contact with outflowing ${ }^{4}$ He gas from the separator, and is further cooled by the outflowing evaporator gas at the second ${ }^{3} \mathrm{He}^{-4}$ He heat exchanger to just above the liquifacticn temperature $\left(3^{\circ} \mathrm{K}\right.$ at 620 torr $)$. It is 1 iquified in the ${ }^{3}$ He Iiquifier by thermal contact with the $2{ }^{\circ} \mathrm{K}$ bath of ${ }^{4} \mathrm{He}$ evaporator. The liquid ${ }^{3}$ He is further cooled to $1^{0} \mathrm{~K}$ by the returning ${ }^{3}$ He gas from the target in the ${ }^{3}$ He counter flow heat exchanger isee Eigure 2.12). It then passes through the ${ }^{3}$ He expansion valve and is cooled to about $0.5^{\circ} \mathrm{K}$ at the target by this expansion. Gas generated in this cooling process is pumped out at 100 torr (or lower) through the $30.5 \mathrm{~cm}$ diamete: tube to the ${ }^{3}$ He pumps. 


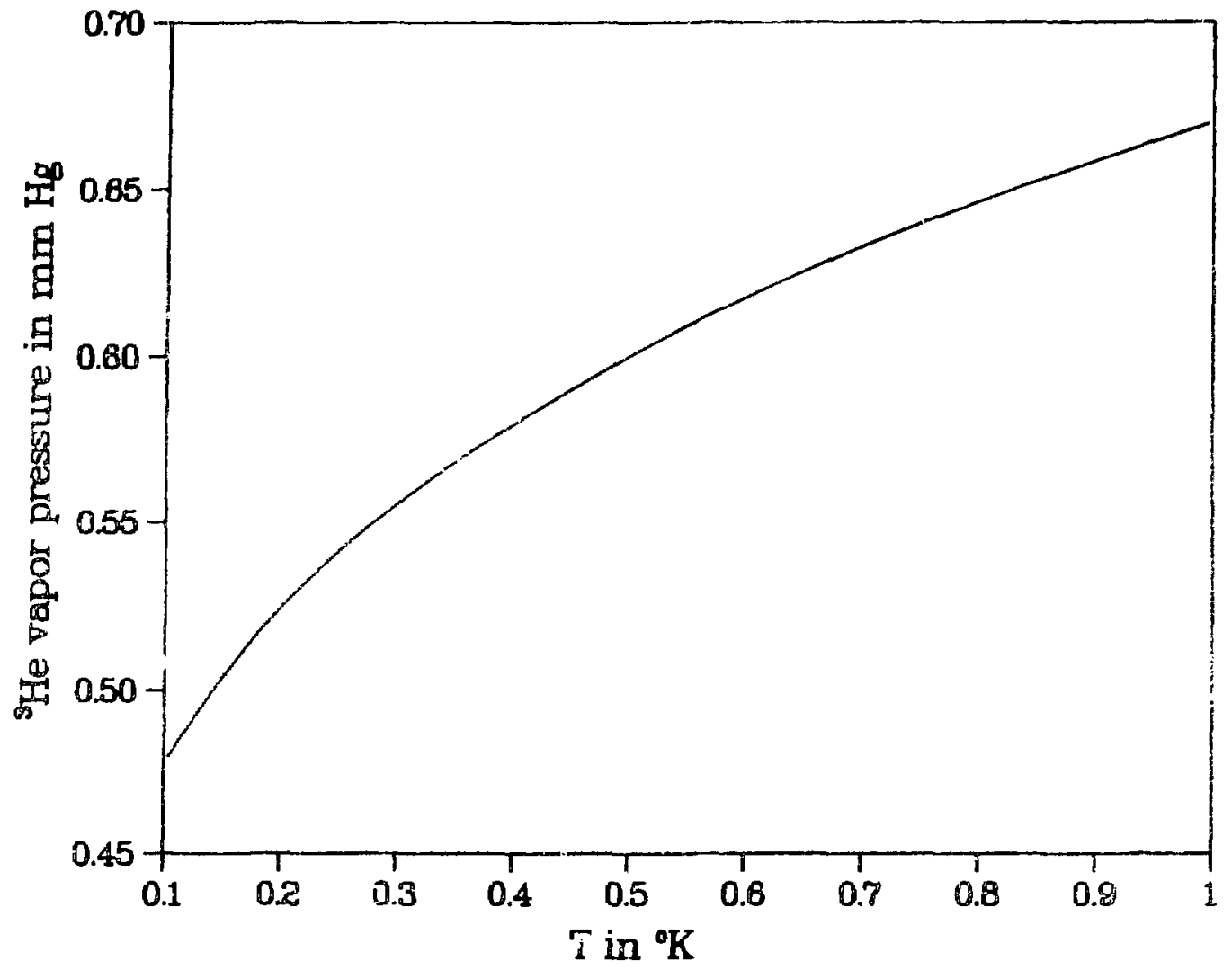

Figure 2.11. ${ }^{3}$ He vapor pressule vs Temperature below $1^{\circ} \mathrm{K}$. 
Figure 2.12. The target cryostat and target insert. The items numbered on the drawing are the following:

1. Separator Valve

2. Separator Tank

3. Evaporator Valve

4. Evaporator

5. Second ${ }^{4}$ He Heat Exchanger

6. First ${ }^{4}$ He Heat Exchanger

7. $10 \mathrm{~K}$ Heat Shieid

8. 50 K Heat Shield

9. First ${ }^{3}$ He Heat Exchanger

10. Second ${ }^{3}$ He Heat Exchanger

11. ${ }^{3}$ He Liquifier

12. Counter Flow Heat Exchanger

13. Pre-Cool Valve

14. ${ }^{3}$ He Valve

15. Target Cell 


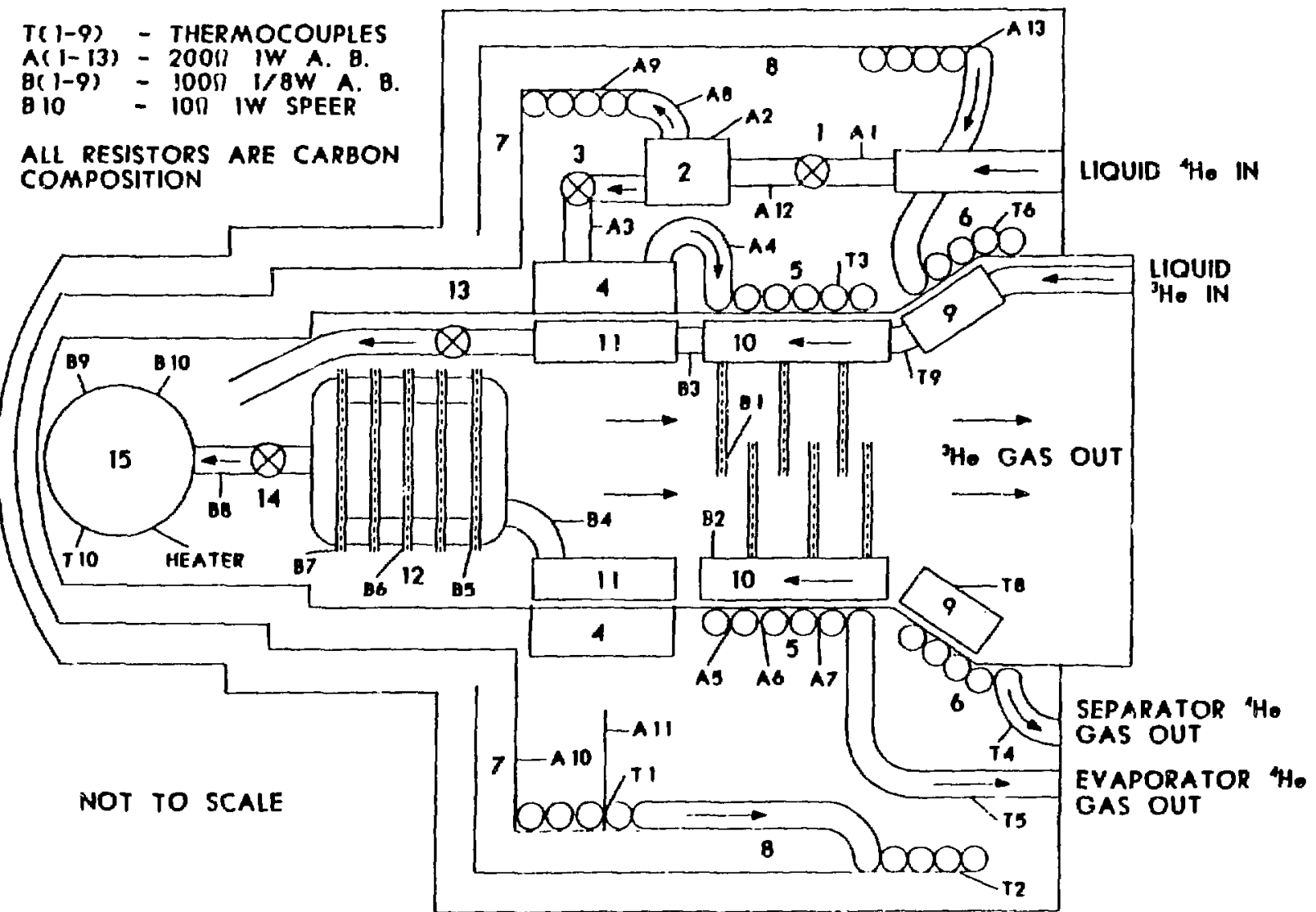




\subsubsection{Cryostat Vacuum}

The cryostat vacuum is needed to insulate the cryogenic systems from roou: iemperature.

\subsubsection{Thermometry}

Nine thermocouples, thirteen $200 \Omega$ carbon resistors, nine $100 \Omega$ carbon resistors, and one $10 ?$ carbon resistor at the target cell monitor the temperature throughout the cryestat and indicate the operating conditions of the target. The target pressure gauge monitors the pressure in the target cell and provides a measure of the target temperature. The location of these devices is shown in figure 2.12 . Measuring the target temperature is especially important in thermal equilibrium runs for the absolute calibration of the NMR polarization monitor using a reference based on Boltzman statistics. The thermocouples are used only during the initial cool-down. The $200 \Omega$, 1 watt Allen Bradley carbon resistors are used in the 2 to $50^{\circ} \mathrm{K}$ range. In the ${ }^{3} \mathrm{He}$ region $100 \Omega, 1 / 2$ watt resistors are used. By having previously measured the temperature-resistance characteristics of a resistor (see figure 2.13), we can determine its temperature by measuring its resistance using sufficiently low currents. Carbon is a semfonductor and its resistance at low temperatures roughly follows the relationship

$\log R \propto \frac{1}{T}$ 


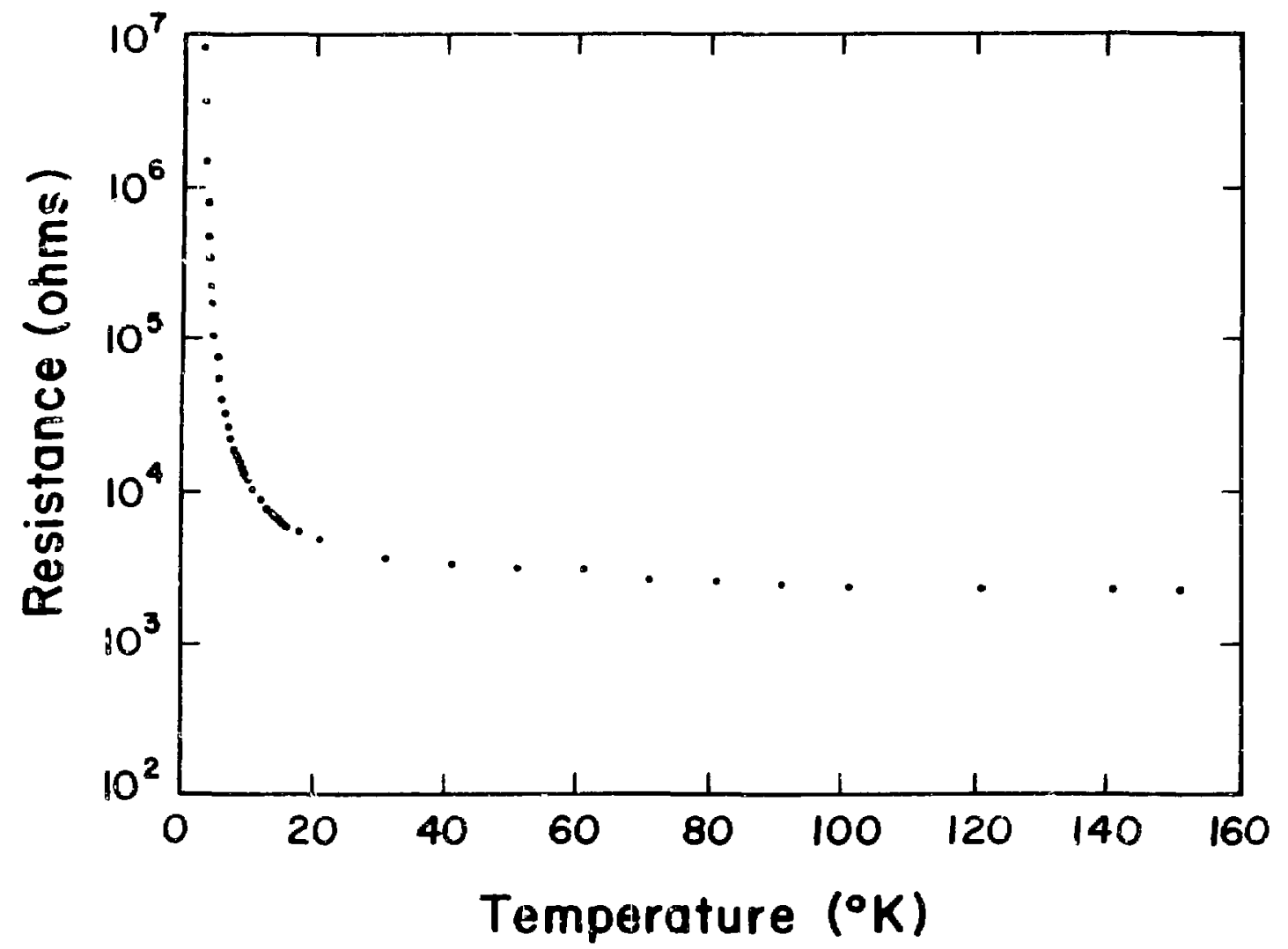

Figure 2.13. Temperature versus resistance curve for an Allen Brarley $100 \Omega, 1 / 2$ watt resistor. 


\subsubsection{Polarized Target Material}

The target material contains free protons which are polarized to a high value by the free electrons of a paramagnetic material. The target material used in this experiment was 1,2-propanediol (PPD, $\mathrm{CH}_{3} \mathrm{CH}(\mathrm{OH}) \mathrm{CH}_{2} \mathrm{OH}$, or $\left.\mathrm{C}_{3} \mathrm{H}_{8} \mathrm{O}_{2}\right)$ to which $\mathrm{Cr}^{5+}$ was added to form paramagnetic complexes. ${ }^{39}$ There are 8 hydrogen atoms per 3 carbon and 2 oxygen atoms, thus the hydrogen percentage in the molecule is $\frac{8}{3 \times 12+2 \times 16+3}=10.53 \%$ by mass. The partial density of free hydrogen is about $0.07 \mathrm{gm} / \mathrm{cm}^{3}$. The target cell consists of a cylindrical copper cavity ( $2 \mathrm{~cm}$ in diameter and $4 \mathrm{~cm}$ long) containing an inner teflon cell which holds the target material and an NMR coil. The material is prepared in the form of beads about $1 \mathrm{~mm}$ dianeter in order to improve thermal contact with the ${ }^{3} \mathrm{He}$ bath and thus dissipate the heat load of the absorbed microwave radiation.

\subsubsection{Microwaves}

$70 \mathrm{GHz}$ microwave radiation transfers the electron polarization to the proton. Changing the microwave frequency by about $0.4 \mathrm{GHz}$ flips the polarization. A reversal of the polarization direction requires about 30-40 minutes. An attenuator allows the microwave power at the target to be adjusted in order to uptimize the polarization. Too little power will not saturate the required transition. Too much power will heat the target beads, reducing the electron polarization (the proton polarization can not exceed the electron polarization). With the microwaves off the heat load on the target becomes low, the 
temperature drops, and the target becomes a "frozen spin target" which will maintain a high polarization for many hours. The microwaves are supplied from a watercooled $400 \mathrm{~mW}$ klystron and transmitted to the target through a waveguide. The frequency may be measured by either of the two wavemeters, which are adjustable resonance cavities.

\section{$2.2 .8 \mathrm{NMR}$}

The NMR (Nuclear Magnetic Resonance) technique is used to monitor and measure the polarization of the target material. The protons populate two levels with energies $\pm_{\mu_{\mathrm{P}}}$. Bonbarding with RF (Radio Frequency) photons of energy $2 \mu_{\mathrm{P}^{B}}$ (which corresponds to a frequency $v=2 \mu_{\mathrm{P}} \mathrm{B} / \mathrm{h}=106.4 \mathrm{MHz}$ ) from the NMR coll in the target induces transitions between these levels. If the populations of the two levels are equal the number of transitions up and down are equal so that no net power is taken from or given to the RF fleld. If the lower energy level has a large population (positive polarization) more transitions are induced upward than downward, absorbing power from the field and giving a dip in the RF signal (see figure 2.14). With a negative polarization power

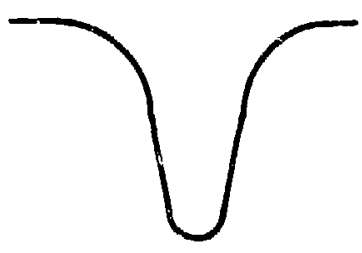

(a)

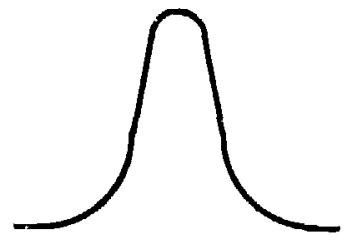

(b)

Figure 2.14. (a) Positive polarization absorb energy from RF field. (b) Negative polarization gives energy to RF field. 
is given to the field and the signal has a hump (see figure 2.14). To first order the size (or more strictly the integral) of the dip or hump is proportional to the polarization.

A multichannel analyzer was used as a frequency analyzer, with the channel number representing the $R F$ frequency and the channel count representing the $\mathrm{RF}$ amplitude. Since changes in the $\mathrm{RF}$ power cause changes in the signal area the RF power was monitored by measuring an RF liode DC voltage. An NMR measurement included a measurement of the RF of the generator, the PTM fleld, the vapor pressure of the ${ }^{3} \mathrm{He}$ surrounding the target and the diode voltage from the NMR coil. The pressure measurement is important only for the thermal equilibrium case since it provides a measure of the target temperature. These data, along with the frequency spectrum were saved in a histogram disk file which can be displayed or accessed by the analysis program.

The NMR apparatus consists of an RF oscillator which drives two Identical circuits one of which drives the NMR coll, the second acts as a dummy for background subtraction. Since the only essential difference is that one coll is in the target, the difference is the NMR signal together with random nolse. 'the difference is amplified by a differential anplifier and stored in a signal averager. The random noise is reduced by the signal averager, which records, displays and integrates the NMR signal. 


\subsection{Detectors And Detection System}

The objective of the experiment was to measure the analyzing power in $\pi^{ \pm}$elastic scattering accirately. This required a high signal-co-noise ratio. Thus the detection system was over-constrained to roject background events, making mis-identification of events nearly impossible. Acceptable events were defined by strong kinematic constraints, TOF requirements, and pulse helght for particle identification. In almos: all measurements the scattered pion and the recoll proton were detected in colncldence using the Large Acceptance Spectrometer, LAS, and a recoll detector. For coincidence events $\mathbf{P}_{S}$, $\theta_{S}$, and $\phi_{S}$ (the momentum, polar, and azimuthal angles of the particle detected in the LAS) together with the polar and azimuthal angles of the particle detected in recoll arm $\left(\theta_{R}, \phi_{R}\right)$ were measured. Because the bend plane is vertical, the particle momentum and scattering angle are determined independently. For the particles detected in the LAS, momentum and time of flight measurements allow unambiguous particle identification. Identification of the particles detected in the recoil arm is accomplished using time of flight with respect to the LAS front scintillator and pulse helght. In a two body final state reaction six degrees of freedom exist. Conservation of energy and momentum provide four relations among these. Thus, the measurement of only two variables, say, $P_{S}$ and $\theta_{S}$, is sufficlent to obtain all the information about the reaction. However, this is not sufficient to reject background from quasi-free scattering, l.e., pion scattering from bound protons in complex nuclei (carbon, oxygen, etc.). Quasi-free 
scatterings are three body final state events. Thus requiring coincident detection with strict two-body kinematics produces a highly efficient background rejection. Because of motion of the protons in the nucleus, the quasi-free events will be kinematically smeared out and will appear, for example, as a broad hump in the coplanarity signal.

\subsubsection{The LAS}

The large acceptance spectrometer 40 consisted of a quadrupole doublet followed by a dipole bending magnet (QQD) with helium gas in the spectrometer drift space to minimize Coulomb multiple scattering along the particle trajectory. The large solid angle of the spectroneter resulted from the large acceptance of the quadrupole pair located close to the target. The LAS incorporates three scintillation counter circuits and four MWPCs with $x$ and $y$ plane wire spacing of $2 \mathrm{~mm}$. A schematic diagram of the LAS showing the magnets and the particle detection system is presented in figure 2.15. The resolution of the system is dictated by wire spacing and is momentum dependent due to multiple scattering effects. The bend plane is vertical (chosen as the $x-z$ plane) with a nominal bend of $30^{\circ}$. The LAS rolls on a leveled semi circle steel track and rotates in the horizontal plane (chosen as the $y-z$ plane) about a vertical axis centered on the target. The horizontal angular acceptance was $9.7^{\circ}$ and vertical angular acceptance $2.4^{\circ}$ giving a solid angle of $29 \mathrm{~ms} r$. The beam design program TRANSPORT ${ }^{36}$ was used to obtain the quadrupole strength so as to achieve a first-order point-to-point transformation from the target to the 


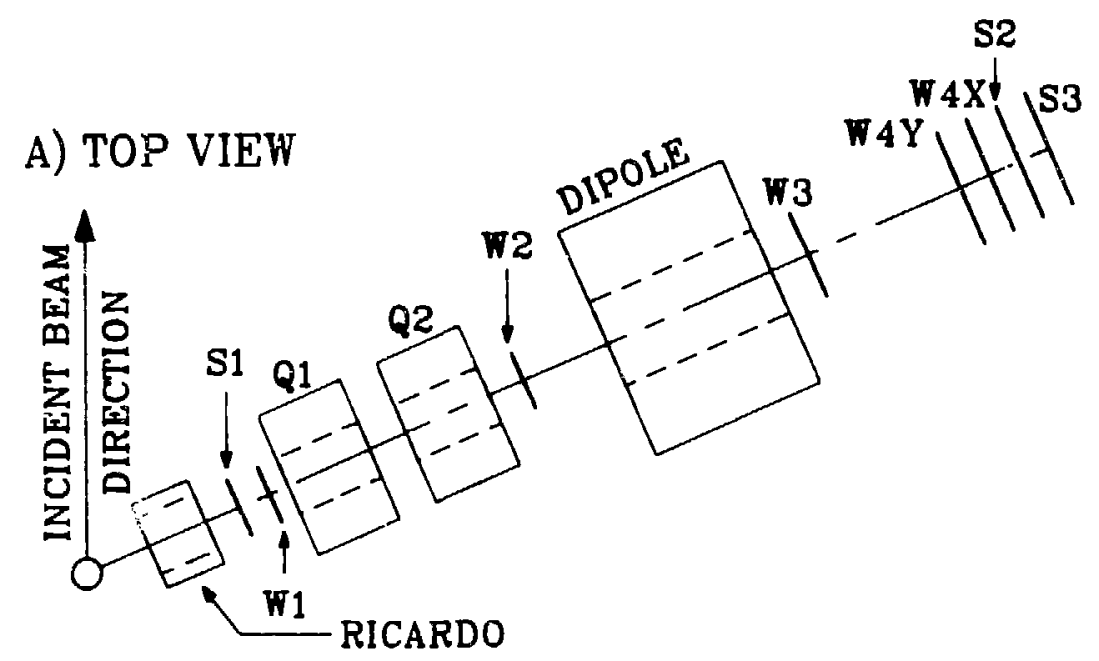

B) SIDE VIEW

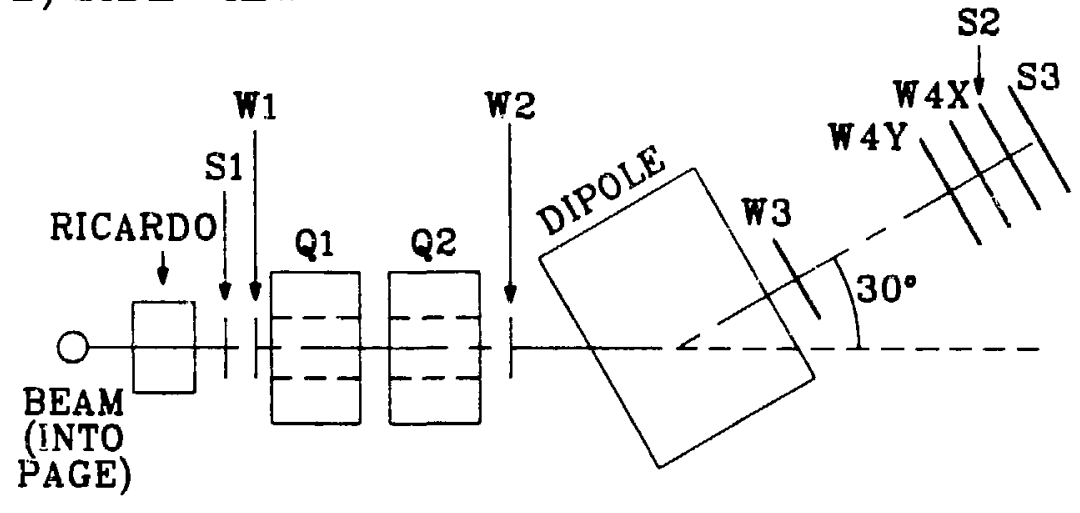

Figure 2.15. The LAS and its particle detection system. 
final MWPC located at a beam flight path of $530 \mathrm{~cm}$. The quadrupoles had an effective length of $50 \mathrm{~cm}$, a bore radius of $15 \mathrm{~cm}$, and a maximum pole tip field of $10 \mathrm{KG}$. Q1 focused in the nonbend plane, while Q2 focused in the bend plane. The dipole had an effective length of $101.2 \mathrm{~cm}$ with a $20.32 \mathrm{~cm}$ horizontal gap. The beam elements are 1 isted In table 2.2. The particle trajectories in the LAS are determined using wire chambers at the entrance and exit of the quadrupole doublet and a pair of chambers following the bend. A scincillation counter at the spectrometer entrance and a focal-plane scintillation array provided time of flight and pulse height information. The plastic scintillation detectors each measured $50.3 \mathrm{~cm} \times 21.8 \mathrm{~cm} \times 0.63 \mathrm{~cm}$ and were located $23 \mathrm{~cm}$ and $28 \mathrm{~cm}$ behind the focal plane. The spectrometer focus is at chamiver $4 X$. The particle momentum is determined by measuring the angular deflection experienced by the particle in traversing the dipole field. In principle this deflection is established by observing the angles of the orbits entering and leaving the dipole region with the wire chamber pairs W1-W2 and W3-W4. However the quadrupole palr between $w 1$ and $W 2$ complicated the trajectory analysis.

W1 and W2 are Individual wire readout chambers. For these chambers the data readout was done using a Proportional Chamber Operating System ${ }^{41}$, PCOS, a commercially available single wire readout system. The system allows multiple hits in each plane and delivers the centroid and width of each hit to a CAMAC crate. The gas mixture for these chambers was about $70 \%$ argon, $30 \%$ isobutane, and less than a 
Table 2.2. Beam elements for the LAS.

\begin{tabular}{|c|c|c|}
\hline Element & Description & $\begin{array}{l}\text { Effective } \\
\text { Length }(\mathrm{cm})\end{array}$ \\
\hline Target & & - \\
\hline Ricardo & Horizontal bend & 22.1 \\
\hline Drift & & 4.2 \\
\hline$S 1$ & Scintillator & - \\
\hline Drift & & 3.9 \\
\hline MWPCl & Wire Chamber & - \\
\hline Drift & & 12.1 \\
\hline Q1 & Forizontal Focus & 50 \\
\hline Drift & & 11 \\
\hline Q2 & Horizontal Defocus & 50 \\
\hline Drift & & 24.6 \\
\hline MWPC2 & Wire Chamber & - \\
\hline Drift & & 43.7 \\
\hline Dipole & Bend $30^{\circ}$ Up & 101.2 \\
\hline Drift & & 50.6 \\
\hline MWPC3 & Wire Chamber & - \\
\hline Drift & & 101.6 \\
\hline MWPC4Y & Wire Chamber & - \\
\hline MWPC4X & Wire Chamber & - \\
\hline s2, s3 & Scintillator & - \\
\hline
\end{tabular}


percent (0.4\%) freon. A charged particle traversing the gas mixture produces a controlled breakdown signal which appears on adjacent wires. The freon gas acts to quench the charge multiplication while the other two gases are involved in amplification.

W3 and W4 are delay line chambers. In these chambers the position at which the particle passes through is determined by measuring the time interval between pulses reaching two ends of a delay line to which the wires are attached. A summary of the LAS wire chamber dimensions is given in table 2.3. Wire spacing is 2 mm in every plane in the LAS

Table 2.3. The LAS wire chamber dimensions.

\begin{tabular}{lcc}
\hline Chamber & Actual Size & Number of \\
& $(\mathrm{cm})$ & Wires \\
\hline $1 X$ & 6.4 & 32 \\
$1 \mathrm{Y}$ & 25.6 & 128 \\
$2 \mathrm{X}$ & 38.4 & 192 \\
$2 \mathrm{Y}$ & 25.6 & 128 \\
$3 \mathrm{X}$ & 50.0 & - \\
$3 \mathrm{Y}$ & 40.0 & - \\
$4 \mathrm{X}$ & 100.0 & - \\
$4 \mathrm{Y}$ & 50.0 & \\
\hline
\end{tabular}


except $4 \mathrm{Y}$ in which it is $4 \mathrm{~mm}$. The gas mixture and the gas flow rate in delay line chambers was the same as in the two front PCOS chambers.

The key information derived $E$ rom the wire chamber coordinates is the LAS particle's momentum. If the spectrometer central momentum is denoted by $\mathrm{P}_{0}$, then the particle's momentum is given by

$$
p=p_{0}(1+\delta)
$$

where

$$
\delta=C_{1} \times 1+C_{2} \times 2+C_{3} \times 3+C_{4} \times 4
$$

The coefficients were first estimated using TRANSPORT. They were then adjusted by trial and error to give minimum peak width and mirimum variation in the peak energy as $p_{0}$ was varied. 42 These coef ${ }^{2}$ icient.s are 1isted below:

$$
\begin{aligned}
& C_{1}=2.420 \\
& C_{2}=-0.960 \\
& C_{3}=0.650 \\
& C_{4}=0.641
\end{aligned}
$$

To see how good the LAS particle momentum is calculated, the channel was tuned for $427 \mathrm{MeV} / \mathrm{c}^{-}$beam and the LAS was set at $0^{\circ}$ while the polarized target magnet was turned off and no target was in the beam. Putting in narrow slits to keep the beam Intensity low, the calculated LAS momentum was compared with the channel momentum

$$
P=427.3 \mathrm{MeV} / \mathrm{c} \quad \text { (Centroid) }
$$


and we got a good focus at the back planes

$$
\begin{aligned}
& \sigma(4 X)=1.5 \mathrm{~cm} \quad(1.1 \mathrm{~cm} \text { from TURTLE) } \\
& \sigma(4 Y)=4.8 \mathrm{~cm} \quad(4.5 \mathrm{~cm} \text { froin TURTLE) }
\end{aligned}
$$

Also the target projections looked reasonable

$$
\begin{aligned}
\sigma(\mathrm{TGTY}) & =0.9 \mathrm{~cm} \quad \text { (Horizontal projection) } \\
\sigma(\mathrm{TGTX}) & =1.0 \mathrm{~cm} \quad \text { (vertiçal projection) }
\end{aligned}
$$

Then the channel was tuned for $427 \mathrm{MeV} / \mathrm{c}^{+}$beam. Same conditions as for $\pi^{-}$beam, the LAS at $0^{\circ}$, PTM off, and no target. The beam was a little over focused in $Y$

$$
\begin{aligned}
& \sigma(3 Y)=4.1 \mathrm{~cm} \\
& \sigma(4 Y)=5.7 \mathrm{~cm}
\end{aligned}
$$

and very sharp in $X$

$$
\begin{aligned}
& \sigma(3 x)=1.3 \mathrm{~cm} \\
& \sigma(4 x)=0.7 \mathrm{~cm}
\end{aligned}
$$

and the LAS momentum spectruin was centered at.

$$
P=427.3 \mathrm{MeV} / \mathrm{c}
$$

with a full width at half maximum of

$$
\sigma(P)=2.5 \mathrm{MeV} / \mathrm{c} \text {. }
$$

\subsubsection{The Recoil Detector}

The recoil arm consisted of a multiwire proportional chamber (MWPC) sandwiched between two planes of elght scintillation counters each. Pulse height, time of flight and wire numbers were recorded for each particle ietected in the recoil detector. The detector was mounted on a movable cart. The cart could be moved, positioned and 
locked at any preset position. Positioning was achieved using a piumb bob, which hung from lower Erame of the cart, and corresponded to the center of the MWPC, over a mark on the floor.

The eight front counters were each consisted of a solid scintillator $0.64 \mathrm{~cm}$ thick, $13 \mathrm{~cm}$ wide, and $71 \mathrm{~cm}$ long coupled via plastic light pipe to an RCA 8575 rhotomultifler = The countis overlapped slightly to cover the entire acceptance of the MWPC. The chamber had an active area of $61.6 \mathrm{~cm}$ by $93.6 \mathrm{~cm}$ with $4 \mathrm{~mm}$ resolution vertically ( 160 horizontal wires) and $8 \mathrm{~mm}$ wire spacing horizontally (120 vertical wires). Because of the large angular acceptance of the spectrometer the recoil detector needed a large angular acceptance. At a distance of $190 \mathrm{~cm}$ from the target, the MWPC had an angular acceptance of $14.2^{\circ}$ horizontally and $9.6^{\circ}$ vertically, giving a solid angle of $170 \mathrm{msr}$. The chamber is an individual wire readout chamber which was operated at 3100 volts. The gas mixture was $70 \%$ argon bubbled through liquid methylal at $0^{\circ} \mathrm{C}$ and $30 \%$ isobutane. The chamber operated at atmospheric pressure and the flow rate displaced the volume of the chamber approximately every three hours.

The chamber was backed by a hodoscope cunsisting of eight double ended scintillation counters. The counters, each $1.27 \mathrm{~cm}$ thick, $15 \mathrm{~cm}$ wide, and $81 \mathrm{~cm}$ long were mounted with approximately $0.5 \mathrm{~cm}$ overlap. Coincldence registers were used to record which counters had fired. Two front counters, two up ends of the back counters, and two down ends were fanned together. The time of flignt and pulse height information 
were sufficient to clearly distinguish pions from protons in the recoil array. The recoll detector is shown in figure 2.16 .

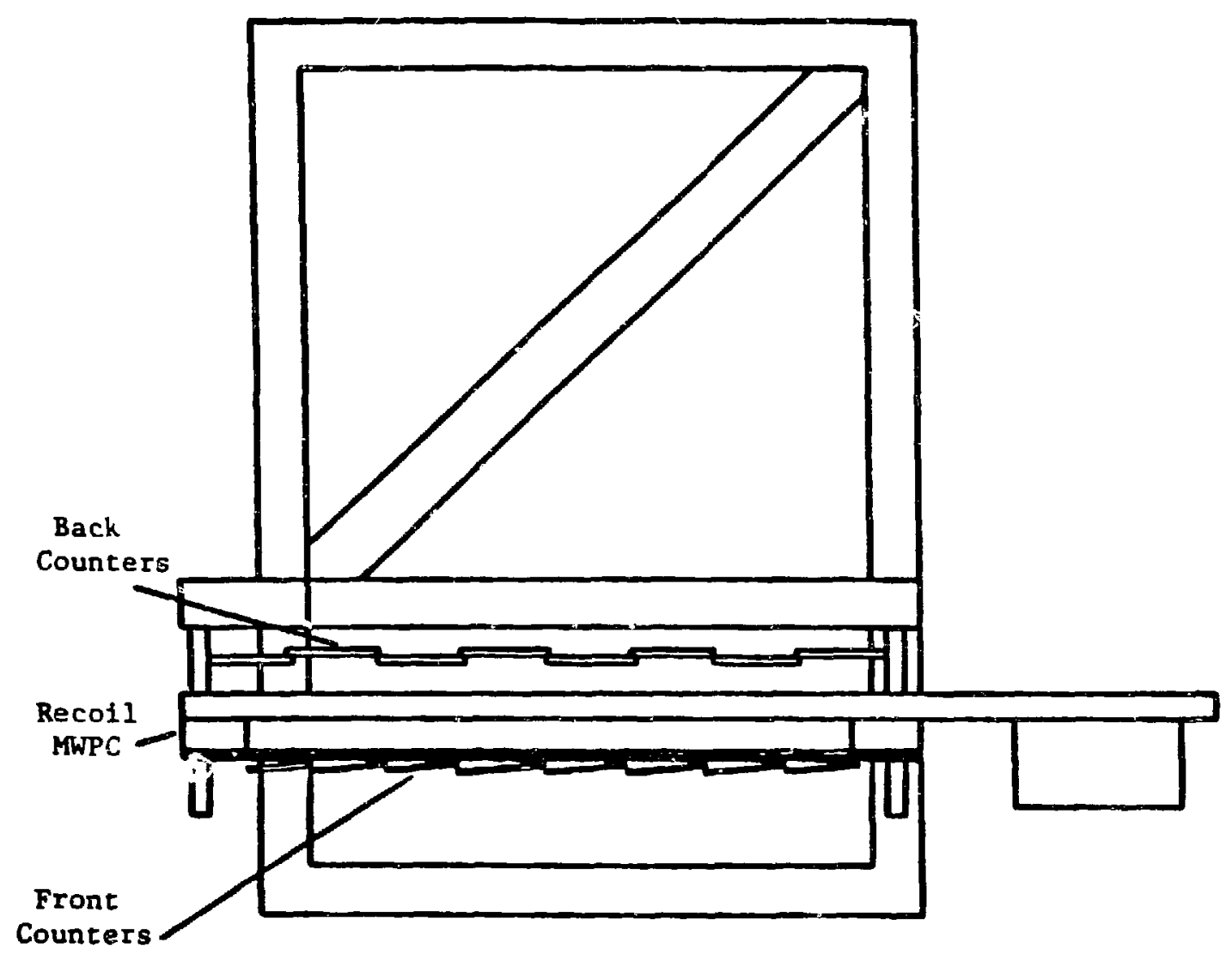

Figure 2.16. Recoil detector and its stand. Top view. 


\subsection{The Electronics}

The electronics provided event definitions and event tiiggers to the computer for the colncidence event and several other types of events. The fast electronics used in the experiment may be divided into four sections:

1) The LAS electronic circuit,

2) The recoil detector electronic circuit,

3) The inhibit electronic circuit,

4) The coincidence event trigger electronic circuit.

Each of these will be outlined. Even though the varlous functions interact with oach other, it 18 easier to understand the overall logic if the above divisicns are made.

\subsubsection{The LAS Electronic Circuit}

A charged particle traversirg S1, S2, and S3 produced a current pulse in each of the associated photomultiplier tubes. Each phototube drove a discriminator whose threshold was set above the noise level ( $\sim 50 \mathrm{mv})$. A three-fold fast coincidence among the logic pulses from the plastic scintillation counters S1, S2, and S3 produced a NIM standard negative $\log 1 \mathrm{c}$ pulse $\mathrm{S} 1 \cdot \mathrm{S} 2 \cdot \mathrm{S} 3$.

S2 consisted of five scintillator counters S2A, S2B, ..., S2E each with its own phototube. The logical "OR" of these was called S2:

$$
\mathrm{S} 2=\mathrm{S} 2 \mathrm{~A}+\mathrm{S} 2 \mathrm{~B}+\cdots+\mathrm{S} 2 \mathrm{E}
$$




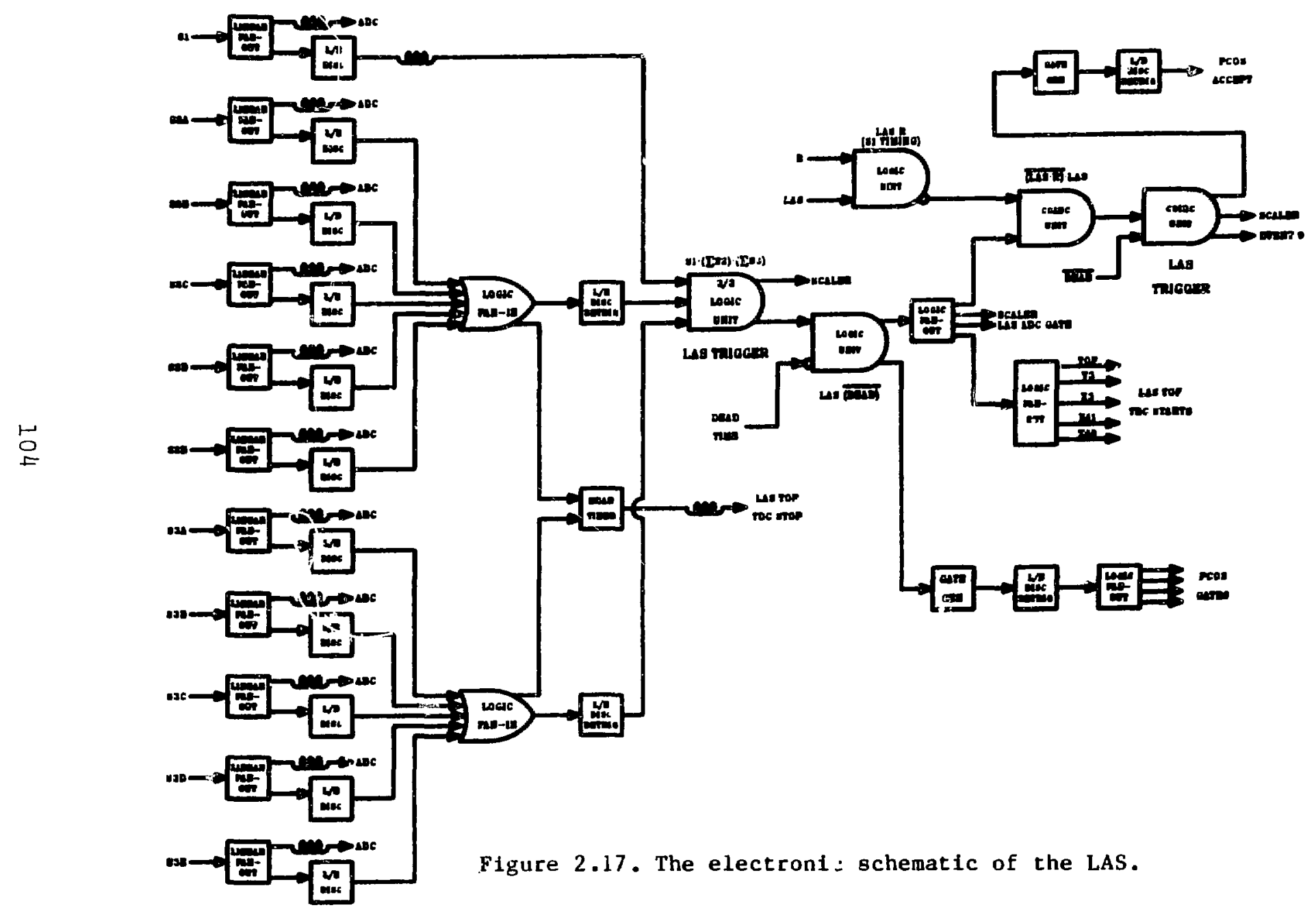


where "+" stands for the logical OR of the signals. Also S3 consisted of five counters with

$$
\mathrm{S} 3=\mathrm{S} 3 \mathrm{~A}+\mathrm{S} 3 \mathrm{~B}+\cdots+\mathrm{S} 3 \mathrm{E} \text {. }
$$

The pulses from S1, S2A, ..., S3E were sent to leading edge discriminators. The two outputs from $\mathrm{S} 2$ and $\mathrm{S} 3$ were put into a mean timer which gave an output pulse at the mean time of the two input pulses. The output from the mean timer provided the time-of-flight (TOF) "stop" signal for the LAS time-to-digital converter (TDC). The start came from the LAS trigger. The LAS trigger consisted of $\mathrm{S} 1 \cdot \mathrm{S} 2 \cdot \mathrm{S} 3$ in colncidence with $\overline{\mathrm{DEAD}}$. The "." symbol stands for the logical "AND" of the signals. The DEAD TIME electronic circuit will be discussed later. No MWPC was in the trigger. The LAS trigger also provided the start for the LAS delay line chamber TDCs and gate pulses for the PCOS chambers and the LAS analog-to-digital converters (ADC). The TDC stops were provided by delay line end pulses. The macro-pulse structire of the beam was used as a regular tick of a clock and was scaled.

\subsubsection{The Recoil Detector Electronic Circuit}

The front scintillator anode signals from the phototubes were split into an $\mathrm{ADC}$ pulse and a discriminator pulse in a 1 to 2 ratio. The back scintillator anode signals from up and down phototubes were split into an $\mathrm{ADC}$ pulse and a discriminator pulse in a 1 to 1 ratio. In order to reduce the number of ADCs required, the ADC signals from front counter pairs 1 and 5,2 and 6,3 and 7 , and 4 and 8 were Fanned together. However, each individual counter had its own discrininator. 


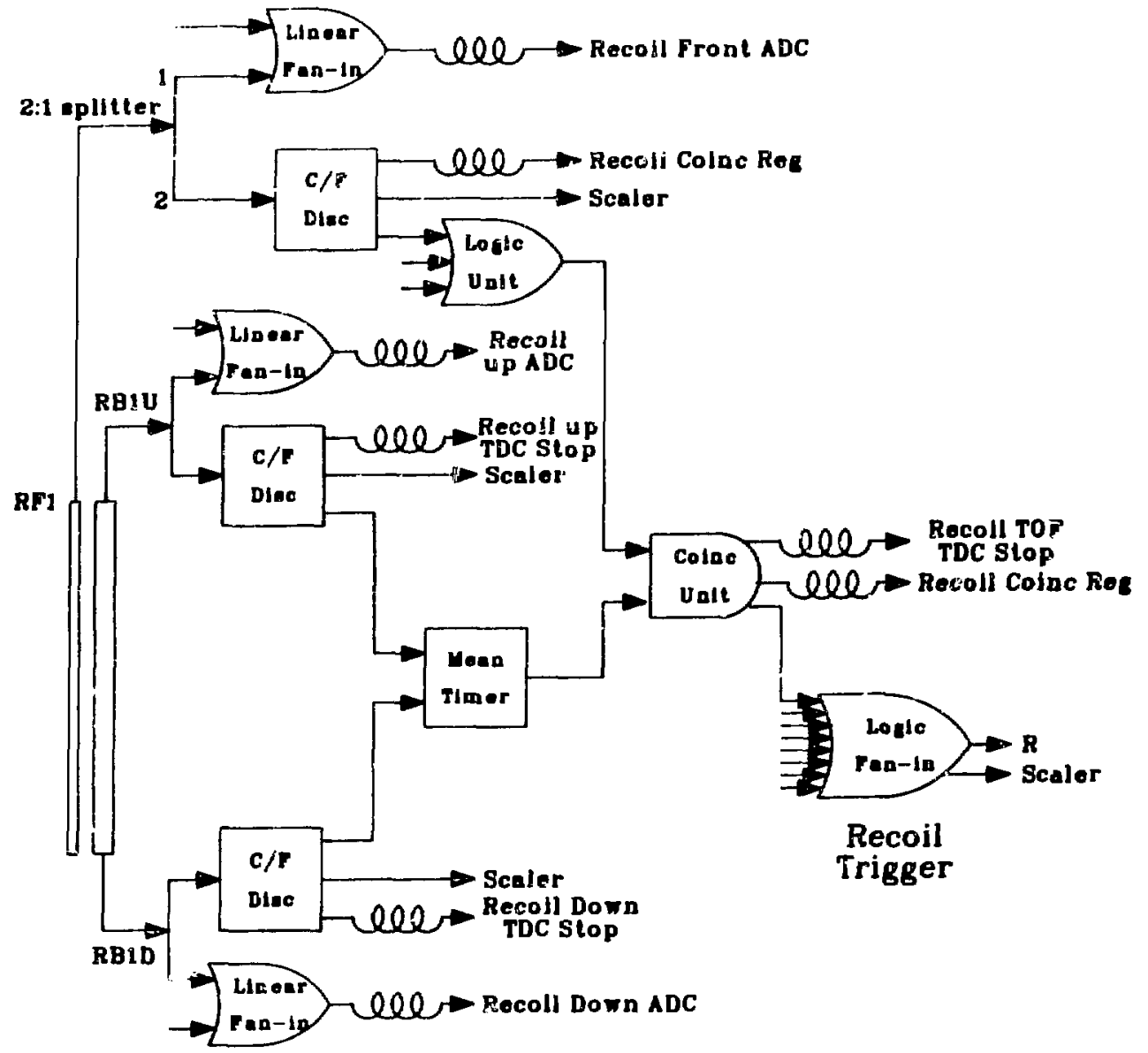

Figure 2.18. The electronic diagram of the recoil detector. 
A coincidence register, which was gated by the event trigger gave a 1 for every signal above the discriminator threshold, and a 0 for the signals which were below. Thus the colncidence register indicated which counter had fired. For the two-ended back scintillation counters the up and down anode pulses were sent to constant fraction discriminators (CFD) providing better time resolution. The two outputs were put into a mean timer, thus eliminating time jitter due to variation in location where the particle passed through the scintillator. The output of the mean timer corresponding to counter $i$ was put in coincidence with the logical $O R$ of front counters $i-1,1$, 1+1. This is the recoil counter trigger circuit. Again the recoil MWPC was not included in the trigger. For each particle detected in the recoll detector the pulse height in the front and back counters and timing information were recorded. In the back counters the time of arrival of the signal at each end of the counter, relative to the event trigger time, was recorded. TOF with respect to Sl (the front counter of the LAS) was also measured. Here the start came from a $L A S \cdot R \cdot \bar{D}$ trigger with S1 timing, and the stop from the individual mean timers. See figure 2.18 for details. The recoll MWPC information was also recorded. Scalers recorded the rates of the individual scintillation counters and wire chamber planes. 


\subsubsection{The Inhibit Electronic Circuit}

The expressions "inhibit electronic circuit" and "dead time generator" are used interchangably and indicate situations under which we do not accept data. The LAMPF Gate Generator (LGG) provided a run gate flip-flop and permitted the control of start and stop of the data runs. The LGG could be controlled by the computer through a CAMAC Kinetic System input gate KS3420 module. The accelerator generated a logic pulse (beam gate) which was dvailable to the experimenter. The beam gate signal was on during a beam macropulse. A "computer busy" signal came from the LAMPF Event Trigger Module. It was set by the event latch and terminated by the computer when the processing of the event was complete. Once the computer read began, the trigger module sent out an inhibit pulse until all modules were read, a process which took about 400 Hs. The CAMAL electronics and the computer were gated off using the LAMPF beam gate, the run gate and computer busy signal as shown in figure 2.19 , insuring that no data would be accepted when the accelerator was not on, the run gate was off or the computer was busy.

For a data run the computer was initialized and events were then accumulated during a run gate. The conputer was signaled to process the event. It subsequently read and cleared the CAMAC registers and added the event to the appropriate histogram. The dead-time pulse ensured that no further gate pulse could reach the registers while the event was being processed. The fraction of events lost during dead time was determined by counting the $\mathrm{Sl} \cdot \mathrm{S} 2 \cdot \mathrm{S} 3$ signals in CAMAC scalers with and without a dead-time inhibit. 


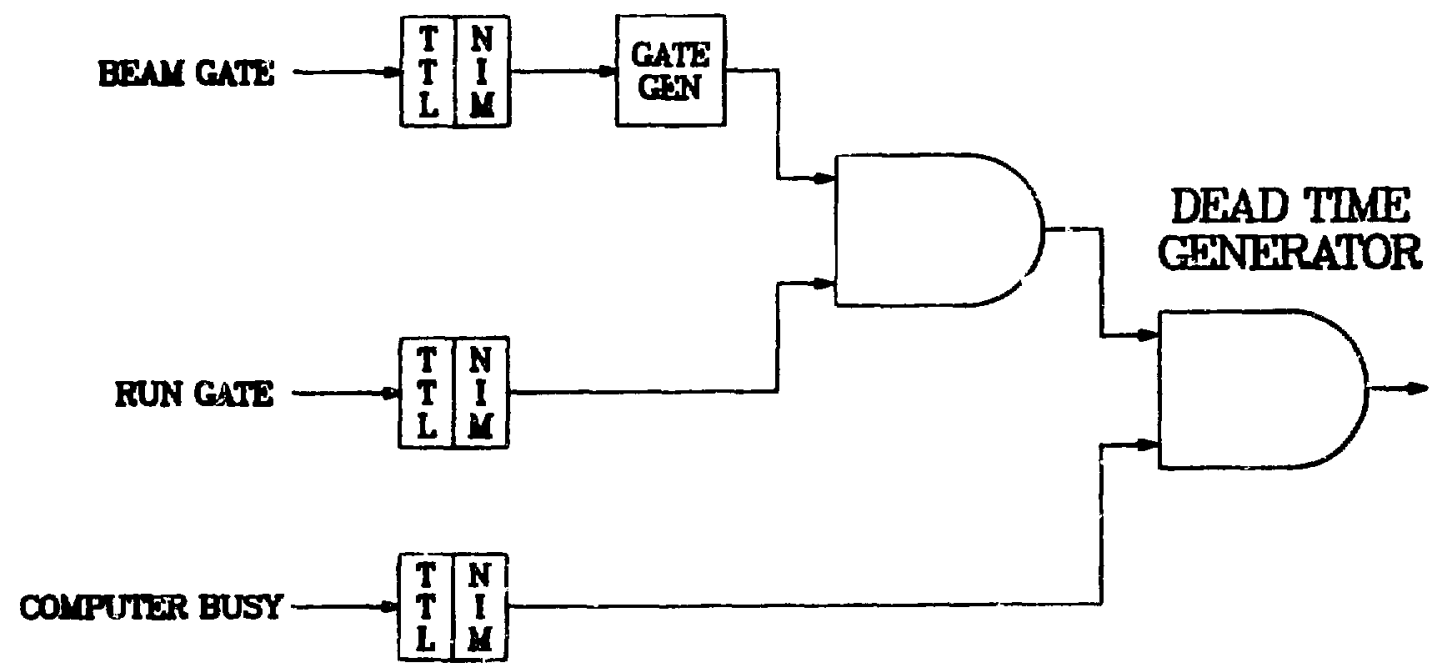

Figure 2.19. Electronic schematic of the dead time generator. 


\subsubsection{The Coincidence Event Trigger Electronic Circuit}

The coincidence event trigger is a coincidence of the LAS and $\mathrm{R}$ in antt-coincidence with the dead time signal:

$\mathrm{LAS} \cdot \mathrm{R} \cdot \overline{\mathrm{D}}$.

A single arm event was defined as:

$$
\overline{(\operatorname{LAS} \cdot R)} \cdot \operatorname{LAS} \cdot \bar{D} \text {. }
$$

The event trigger signal is fed into the LAMPF trigger module to initiate reading of the event, it also provides the start for the TDCs and gate pulses for the ADCs, coincidence register and $\bar{Y} C u$ s chambers. The coincidence event trigger electronic circuit is shown in figure 2.20 . 


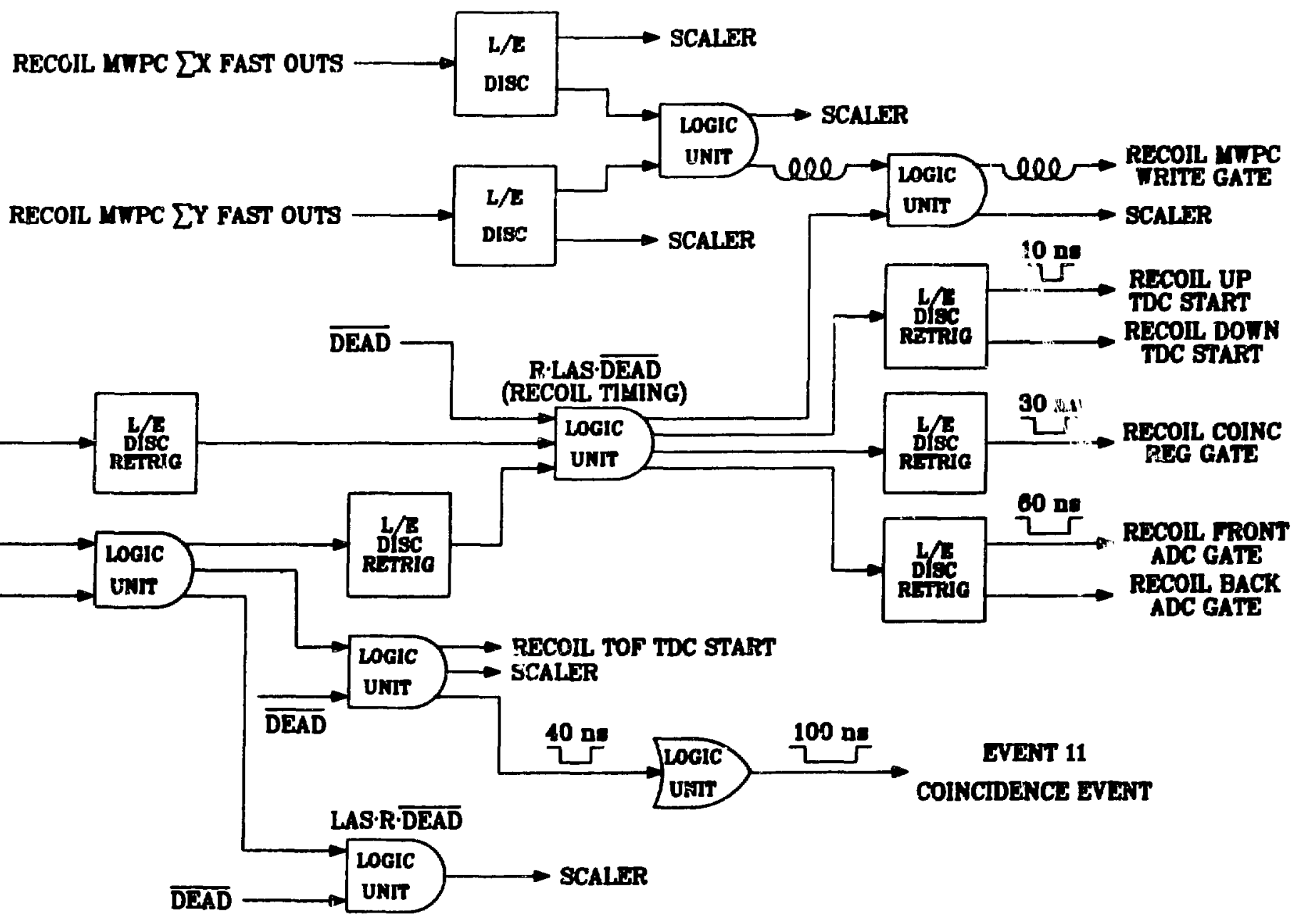

Figure 2.20. Electronic schematic of a coincidence event. 


\subsection{Data Acquisition and Software}

The data acquisition components consisted of the computer, the Micro-programmable Branch Driver (MBD), and the CAMAC and NIM electronic modules. A Digital Equipment Corporation (DEC) PDP-11/45 minicomputer was used with $124 \mathrm{~K}$ words of memory providing room for histogramming and data processing. The $\mathrm{MBD}^{43}$ is a small computer which handles the transfer of data from the CAMAC crates to the main computer. The resulting data consisting of 139 16-bit integers for each coincidence event and 47 data words for each single arm event were readout through CAMAC into the $M B D$, which buffered the data until it was released to the computer. The CAMAC modules were reset and the system made active for the next event. Meanwhile the data were transferred from the MBD to the computer memory and subsequently recorded on magnetic tape. The events are routed to the MBD via the LAMPF trigger module. This is a module with a series of inputs numbered 4 to 11 . Whenever a NIM signal is received at the trigger module, the corresponding event is triggered. This experinent used three data events: 6,9 , and 11. Event 6 was the scalers readout event, event 9 the single arm (spectrometer only) event, and event 11 the coincidence event. The single arm event was defined as the detection of a charged particle in the spectrometer, while the coincidence event was the simultaneous detection of charged particles in both spectrometer and recoil detector. Whenever an event was triggered, the data were read, then written to tape. The data record 
included the run number, event number, and the number of data words in the event.

The computer was run in "MAY PROCESS" mode. In this mode all incoming events are written on tape, but an event is processed on-1ine only if time is available (i.e. computer is not busy). The advantages of running in may process mode are obvious-the tape can be replayed later on to retrieve all events recorded, and fewer events are lost due to computer dead time.

The data processing was done with a progran called the EVENT ANALYZER 44 in conjunction with a TEST FILE and a DISPLAY PACKAGE. The event analyzer is the most important part of the data acquisitior. system. It indicated what data should be acquired from CAMAC in response to a particular trigger and what analysis was required on these data. The test file performed a set of logical tests on each event. The tests can be cuts, as well as logical AND or oR combinations of the previous tests. The display package enabled construction and visual display of histograms containing either raw or computed quantities from the analyzer. A histogram can be gated by any test from the test package. 
CHAPTER THREE

THE ANALYSIS

\subsection{Scattering Data Analysis}

One of the great advantages of a two-body final state reaction is that the kinematics are well determined. Virtually all of the software cuts imposed on the data exploit the kinematics of the reaction $\pi^{ \pm} \mathrm{p}+\pi^{ \pm} \mathrm{p}$. This chapter will focus on the handling of the data after a trigger has been accepted as a good event candidate by the hardware. So, in a sense, some of the data selection is already done in the design of the experiment before the data is ever taken. Before data reduction is discussed, let us briefly talk about the kinematics in the presence of the polarized target magnet. With the PTM field up the $\pi^{-}$ beam bends to the left, while it bends to the right when the PTM field direction is down. To extend the accessible final state angular range the incident pion beam went through the PTM yoke. As a result the central ray was slightly shifted at the target position and made an angle relative to the undeflected incident beam line. This angle, which is called the incident beam angle is different from the offset 
angles given in table 2،1. At $471 \mathrm{MeV} / \mathrm{c}$ the incident angle is as large as $18^{\circ}$. This is important in positioning the detectors. All laboratory angles are measured relative to the undeflected incldent beam direction with positive angles indicating left deflection and negative angles right deflection. Center of mass scattering angles are indicated by the subscript $\mathrm{cm}$. Take $\pi^{-} \mathrm{p}$ elastic scattering for the $471 \mathrm{MeV} / \mathrm{c}$ incident beam with the PTM field down at $\theta_{\mathrm{cm}}=62.5^{\circ}$. At this scattering angle $p_{\pi}=406 \mathrm{MeV} / \mathrm{c}, \theta_{\pi}=-46^{\circ}, p_{p}=347 \mathrm{MeV} / \mathrm{c}$, and $\theta_{p}=57^{\circ}$. The LAS first chamber was $75 \mathrm{~cm}$ away from the target. The $406 \mathrm{MeV} / \mathrm{c}$ scattered $\pi^{-}$loses $5 \mathrm{MeV} / \mathrm{c}$ in the target and bends $17^{\circ}$ in the PTM-Ricardo fleld to the right over this distance, therefore the LAS was set at $\theta_{\text {LAS }}=-\left(46^{\circ}+18^{\circ}+17^{\circ}\right)=-81^{\circ}$ with a nominal central momentum of $401 \mathrm{MeV} / \mathrm{c}$. The recoll detector was located $190 \mathrm{~cm}$ away from the

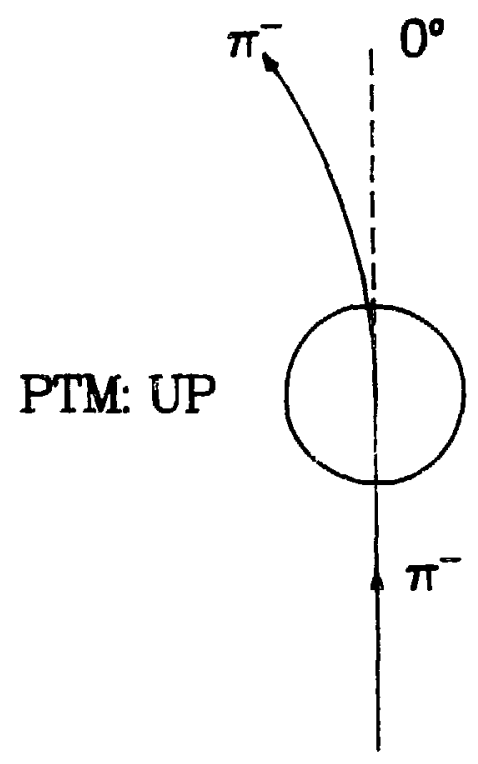

(a)

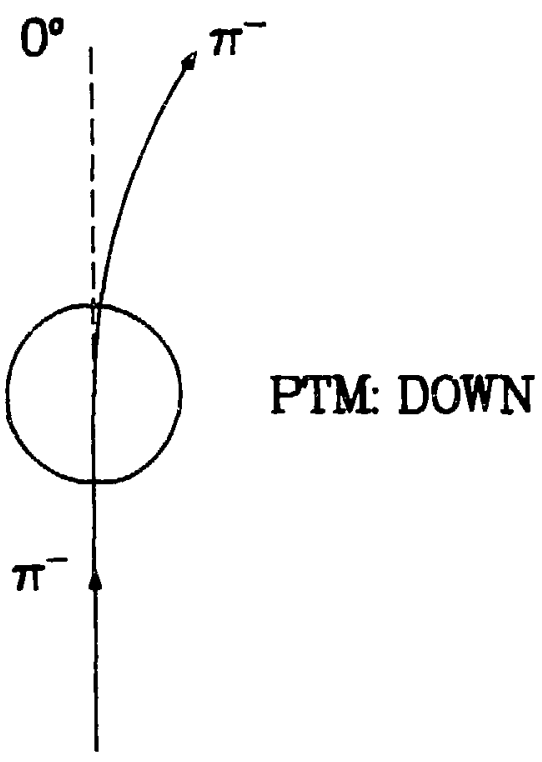

(b)

Figure 3.1. Bending of charged particles in the PTM. When the PTM field is up the $\pi^{-}$beam bends to the left. It bends to the right when the PTM is down. 
target. The $347 \mathrm{MeV} / \mathrm{c}$ recoil protons lose $70-100 \mathrm{MeV} / \mathrm{c}$ in the target cryostat and when they emerge from the target their momentum is only $200-300 \mathrm{MeV} / \mathrm{c}$. The $250 \mathrm{MeV} / \mathrm{c}$ protons bend $34.5^{\circ}$ in the PTM field to the left. Therefore the recoll detector was set at $\theta_{R}=57^{\circ}+34.5^{\circ}-18^{\circ}=73.5^{\circ}$. To extend the available angular range, the polarized target magnet was operated with opposite polarities at different times. In this way, pions scattered at certain angles which would miss the LAS with one setting of the magnet will be deflected Into the LAS with the opposite polarity. These polarity reversals are not required to reverse the direction of the proton polarization. Target polarization reversal is accomplished, at either magnet polarity, by a change in the frequency of the microwave radiation used in the dynanic polarization process. For the $\pi^{+}$p elastic scattering at $471 \mathrm{MeV} / \mathrm{C}$ with the PTM up and at the same center of mass scattering angle of $\theta_{\mathrm{cm}}=62.5^{\circ} ; \mathrm{p}_{\pi}, \theta_{\pi}, \mathrm{p}_{\mathrm{p}}$, and $\theta_{\mathrm{p}}$ are the same as in the previous example. Since the sign of the electric charge of the pion and the direction of the PTM field both have changed, the LAS settings (both central momentum and angle) remain the same. But the recoil protons, unlike in the previous example bend to the right. Therefore $\theta_{\mathrm{R}}=57^{\circ}-18^{\circ}-34.5^{\circ}=4.5^{\circ}$. Because of physical Iimitations the recoil detector was set at $6.6^{\circ}$ in this case.

Since the LAS central momentum is the same, $\pi^{+}$and $\pi^{-}$have the same time-of-flight (TOF). The only difference is that in the $\pi^{-} p$ case, protors having a positive electric charge do not make it through the spectrometer. Figure 3.3 shows the LAS time-of-flight histograms. 


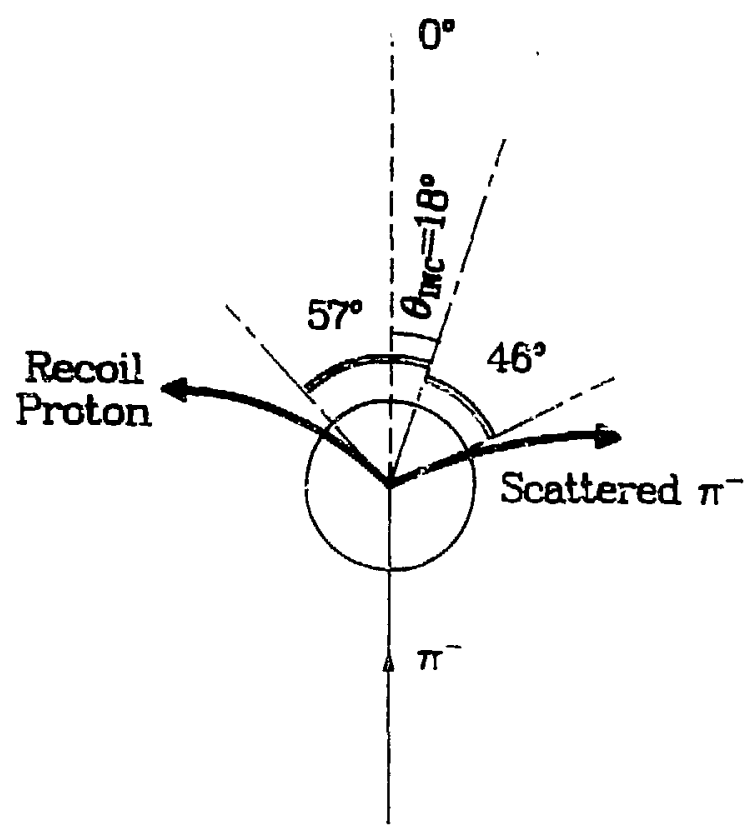

(a)

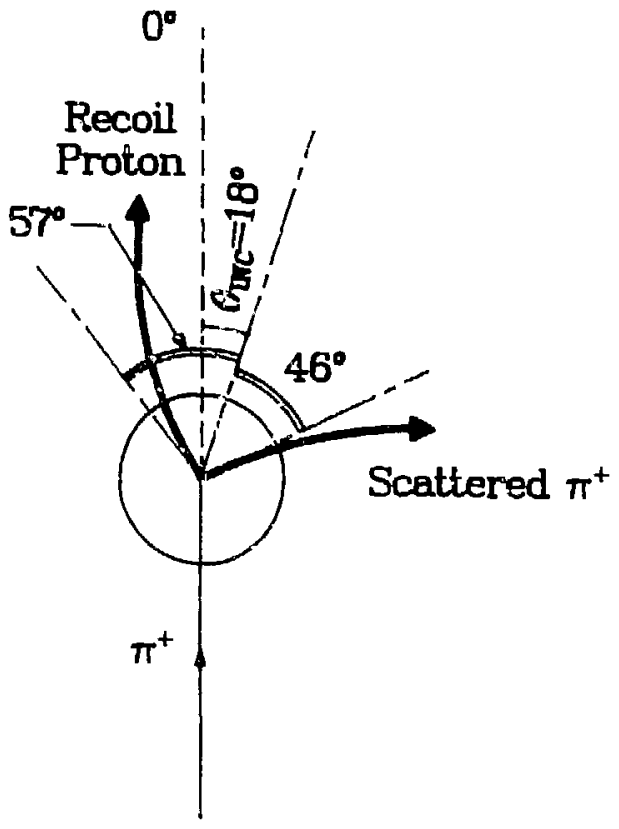

(b)

Figure 3.2. $\pi^{ \pm}$p elastic scattering in the presence of the PTM.

As the distance between $\mathrm{Sl}$ and $\mathrm{S} 2 \cdot \mathrm{S} 3$ is 4.65 meters the time of flight difference between pion and proton is 23 ns. in order to produce histograms which were used to obtain the yields needed to calculate the scattering asymmetries, the scattering data were subjected to cuts. We used the same TOF cuts for $\pi^{+}$and $\pi^{-}$data.

In the case of the $\pi^{+}$data proton-proton inceractions could potentially contribute to the background. The proson contanination of the $\pi^{+}$beam was measured by splitting the beam counter signal in a 1 to 1 ratio. Each signal was fed into a discriminator with different threshold setting. Since protons are heavier than pions they lose more 


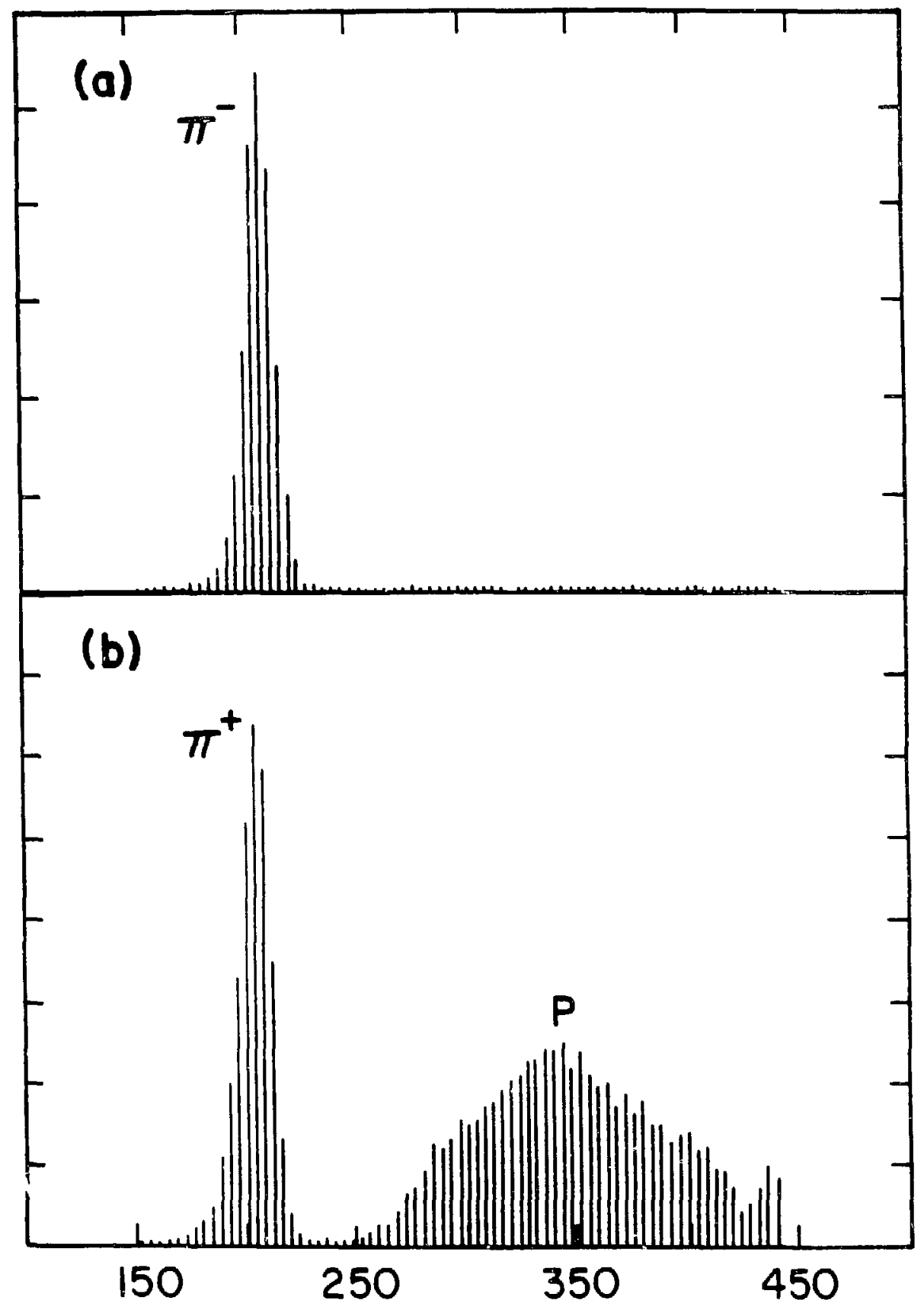

Figure 3.3. TOF in the spectrometer measured between $\mathrm{S} 1$ and $\mathrm{S} 2 \cdot \mathrm{S} 3$. Each channel is 0.2 ns. (a) The LAS tuned for $\pi^{-}$detection. (b) The LAS tuned for $\pi^{+}$detection. 
energy in the beam counter and thus give rise to a bigger pulse height stgnal. In the worst case at $687 \mathrm{MeV} / \mathrm{c}$ the proton contamination was $55 \%$

The proton background was rejected by applying a cut to the TOF spectrum of the LAS. The spectrometer scintillator pulse height were also used to reject the proton background. Cuts on recoil detector pulse height and time of flight between recoil counters and the LAS front scintillator $S l$ also helped to reject the proton contamination. In making the first cut, the spectrometer TOF information was used rather than the LAS counters pulse height, because of the better resolution in TOF. Figure 3.4 (a) shows the LAS front scintillator pulse height spectrum of the raw data and figure 3.4 (b) is the same data after applying the spectrometer TOF cut.

Using the position information provided by the two LAS front chambers the target projections in the vertical and horizontal directions, TGTX and TGTY respectively were obtained. The horizontal target projection, TGTY, although shifted due to the PTM field is still useful to reject the events which did not come from the target. These histograns are shown in figure 3.5.

Another two histograms, HANG and VANG, show the deviations from the central ray between the LAS third and fourth chambers in horizontal and vertical planes, respectively. The deviation is an indication that the track in the LAS is due to a muon. The muon background at the LAS 


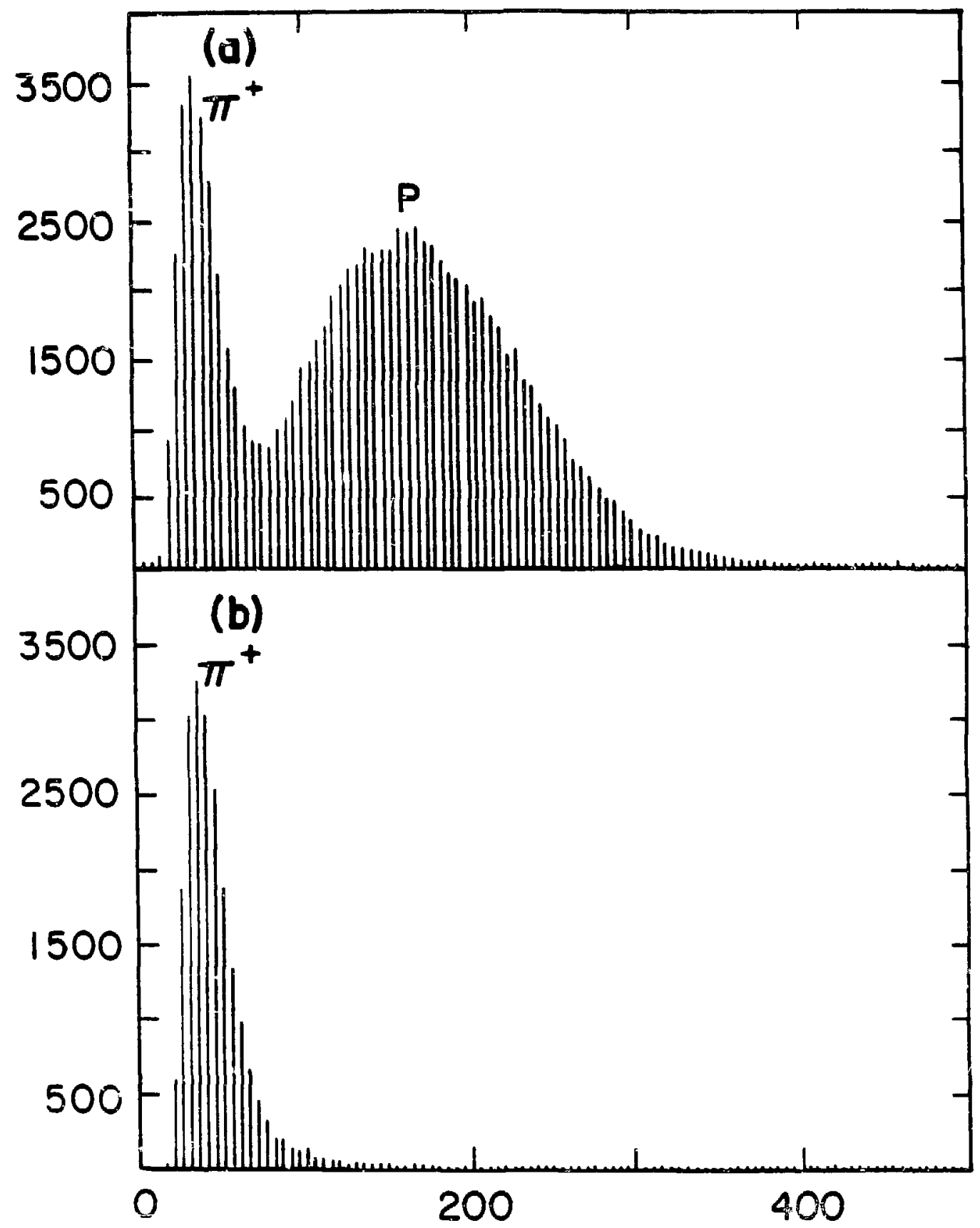

Figure 3.4. The LAS front scintillator pulse helght. (a) Raw data. (b) Same sample after the LAS TOF cuts. 


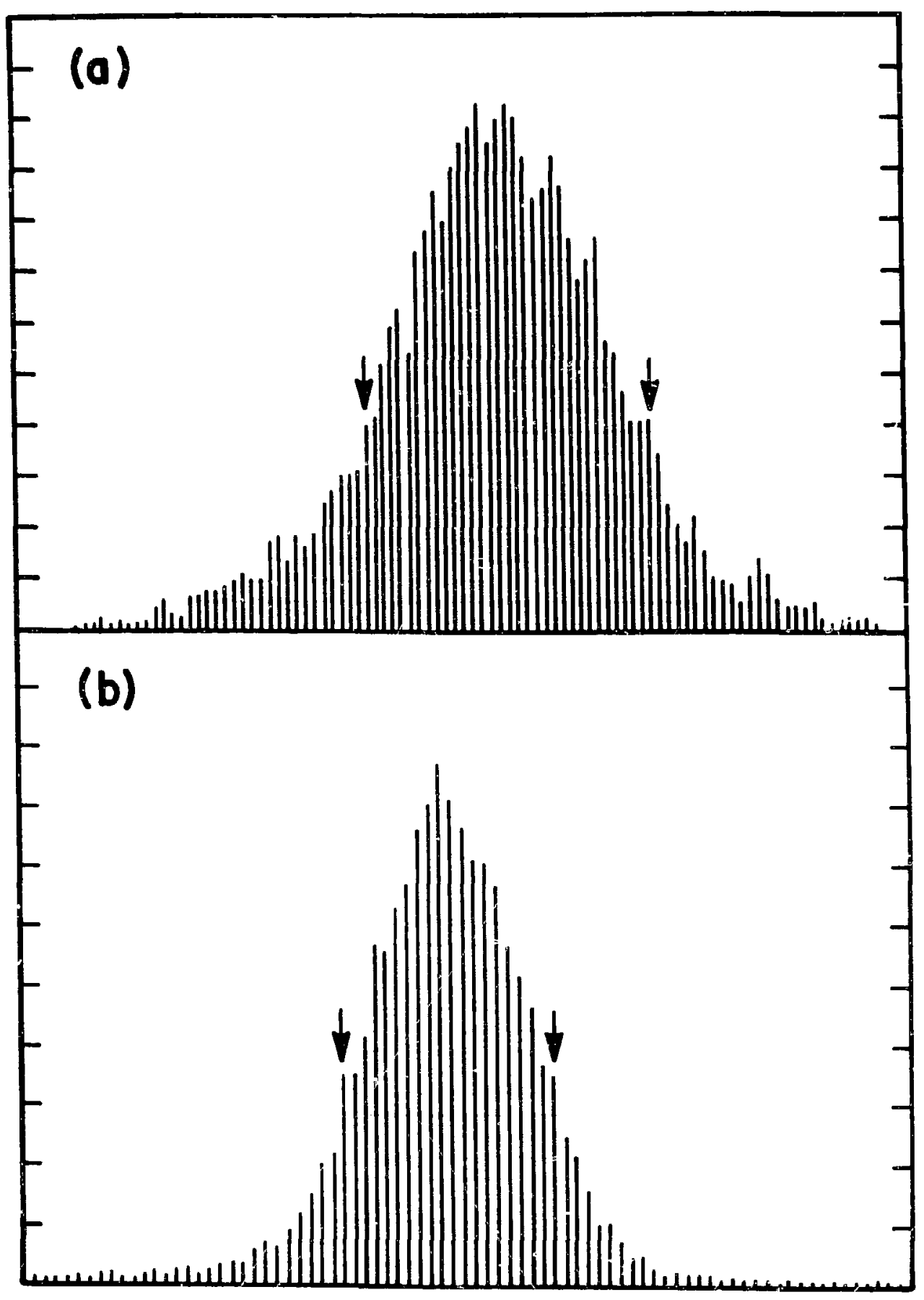

Figure 3.5. (a) Hortzontal target projection. (b) Vertical target projection. Each channel is $1 \mathrm{~mm}$. The arrows Indicate the edges of the target. 
focal plane comes from two sources: beam muons scattered Into the LAS, or muons from pion decays in the LAS. Because muons do not interact strongly, the contributions from the beam muons is expected to he small except at very small angles. The pion decays by an exponential decay law

$$
N=N_{0} e^{-t / \tau}
$$

where $N$ and $N_{0}$ are the number of pions at time $t$ and $t=0$, and $\tau=2.60 \times 10^{-8}$ seconds is the mean life. For particles in flight the mean life $\tau$ in their rest frame is different from that in the laboratory frame $\tau^{\prime}=\gamma \tau$. If the pions have a velocity $v=\beta c$, the number of remainning pions at a distance d from the source is

$$
\mathrm{N}=\mathrm{N}_{0} \mathrm{e}^{-\frac{\mathrm{d}}{\mathrm{Bc \tau \gamma}} \text {. }}
$$

Here $\mathrm{d}$ is the distance froin the target to the focal plane of the LAS. Using $c \tau=780.4 \mathrm{~cm}$ and $d=530 \mathrm{~cm}$, for $401 \mathrm{MeV} / \mathrm{c}$ pions we find $\mathrm{N} / \mathrm{N}_{\mathrm{O}}=0.79$. Muons from pion decay reach the focal plane at different positions with a variety of trajectories, giving rise to a false momentum and thus were rejected by the software cuts on HANG and VANG. The remaining few muons are expected to be randomly distributed across the focal plane and constitute a flat background. Typical HANG and VANG histograms are shown in figure 3.6 .

The recoil arm detectors are incapable of identffying different particles. However, the time-of-flight of individual recoil counters with respect to $S I$ and pulse height information were sufficient to clearly distinguish pions from protons in the recoil detector. A typical TOF histogram is shown in figure 3.7 (a). Figure 3.7 (b) is 


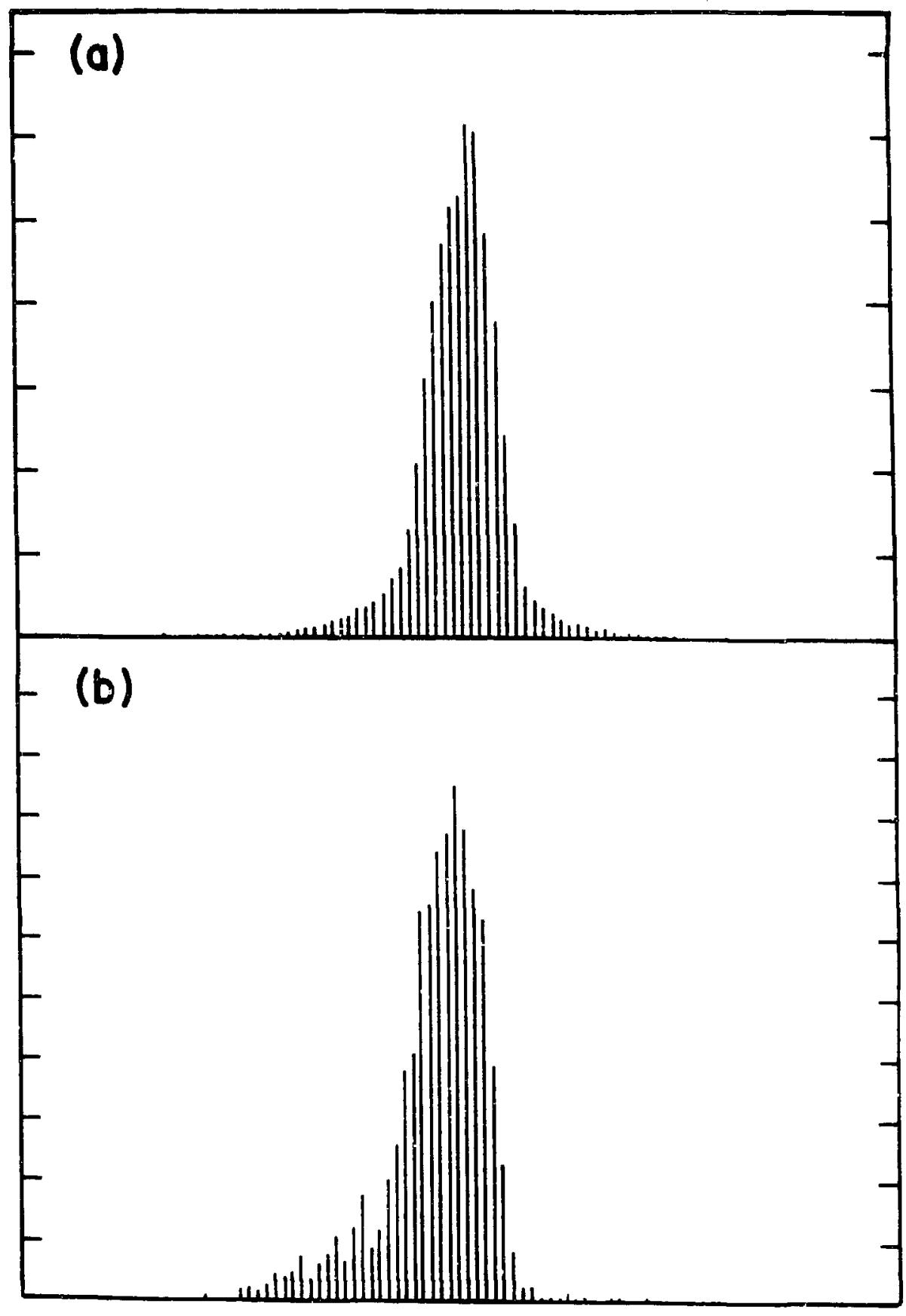

Figure 3.5. Deviations of the tracks from the central ray between the third and fourth chambers in the LAS. (a) In horizontal plane. (b) In vertical plane. Each channel is 0.2 degrees. 
the same as figure $3.7(a)$, only it is gated by the LAS TOF. This histogram helps to reject quasi-elastic events. It is important to reduce this background through the kinematic constraints as much as possible. Figure 3.8 (a) shows a typical recoil pulse height histogram, and again figure 3.8 (b) is the same histogram only gated off by the LAS TOF. In the recoil back counters the time of arrival of the signal at each end of the counter was recorded. The sum and difference of these signals were also histogramed. By applying cuts to the sum the multiple hits were rejected. The cuts on the difference rejected the quasi-elastic background. The difference acted as the coplanarity signal.

The experimental approach used was to measure the number of scattered pions and recofl protons for the two opposite directions of the target polarization. The transition from raw data to the left-right asymetry involved three sets of data at each angle: the Fularization up data, the polarization down data, and the background measurement. The same set of cuts were applied to spin up, spin down, and background runs at each angle. The ylelds were normalized to the beam monitors and corrected for chamber efficlency and dead time. The pion survival fraction over the spectrometer path length and the solid angle are multiplicative factors and remain the same independent of target spin direction, and thus cancel from numerator and denominator of the asymmetry equation. The number of target nucleons per $\mathrm{cm}^{2}$ was the same for spin up and spin down runs, but was slightly different for the background measurement runs and the background was adjusted for 


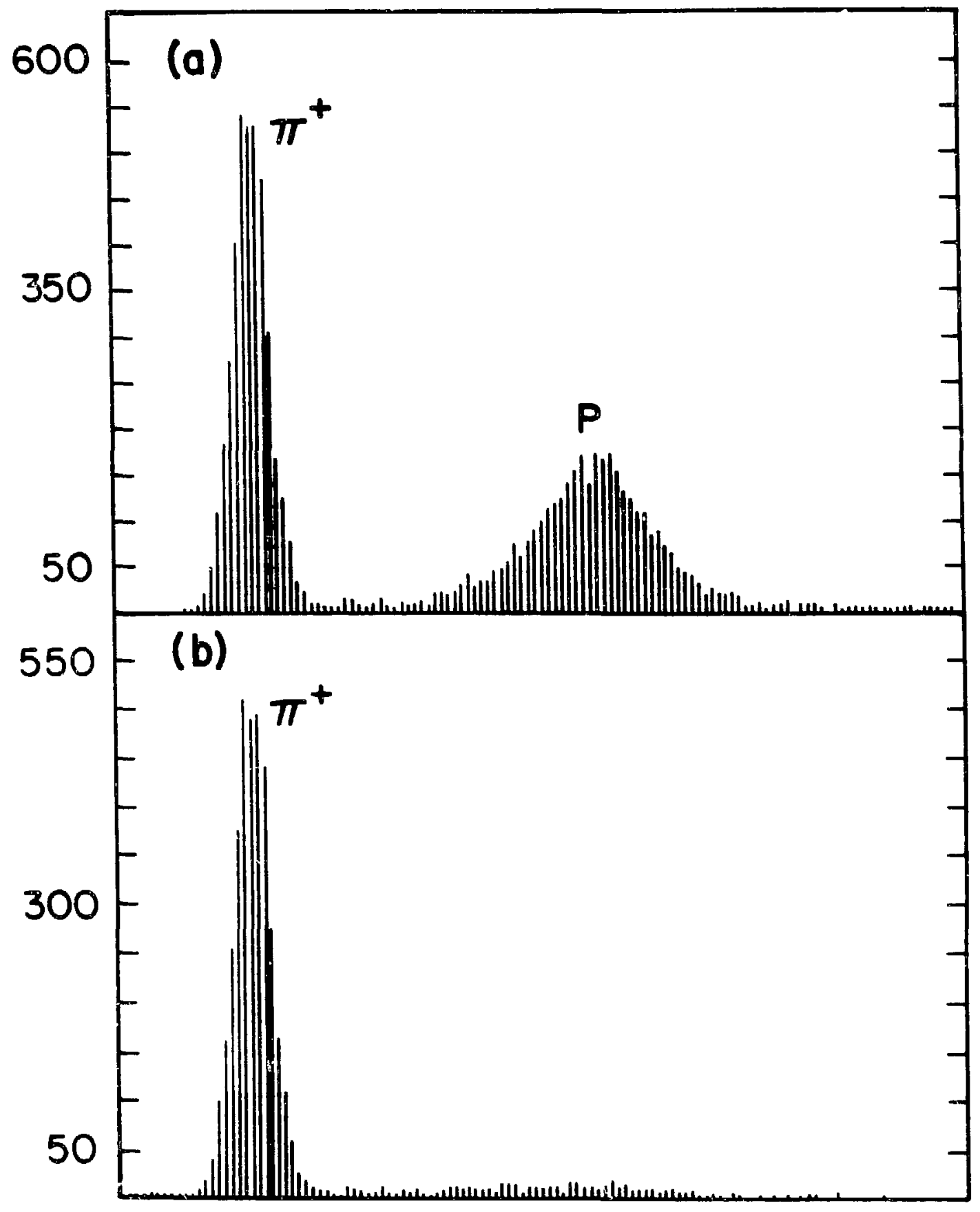

Figure 3.7. Recoil TOF. (a) Raw data. (b) Raw data gated by the LAS TOF. Each channel is 0.2 ns. Protons were detected in the LAS. 


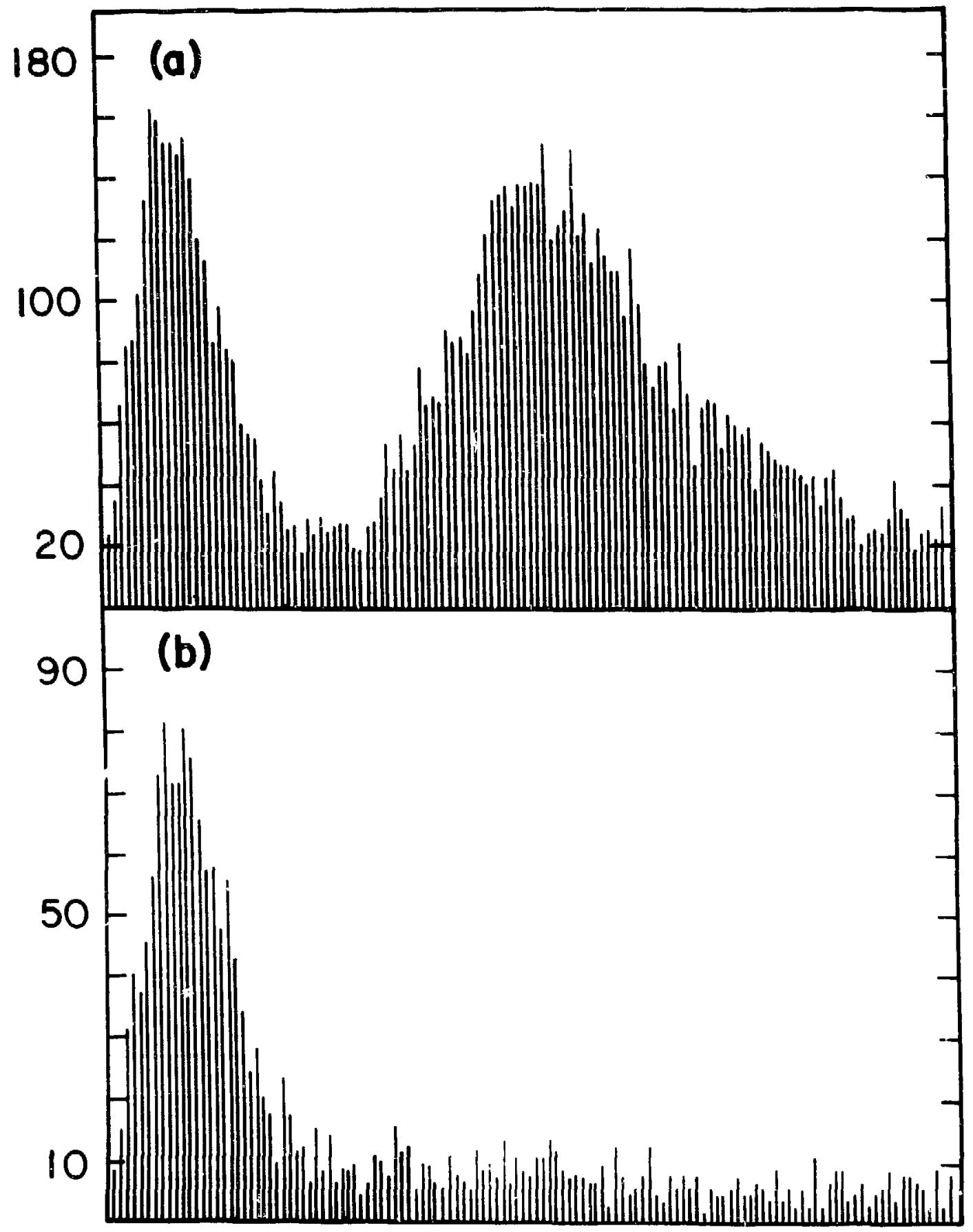

Figure 3.8. Recoil pulse height. (a) Raw data. (b) Raw data gated by the LAS TOF. Protons were detected in the LAS. 
that. A typical LAS momentum spectrum is shown in figure 3.9. The top histogram is the spectrum without the cuts and the bottom one with the LAS TOF, TGTX, TGTY, HANG, VANG and recoll TOF, and recoil chamber cuts.

The efficiency of the LAS wire chambers is defined as the ratlo of colncidences between all the LAS $x$-planes to the number of processed coincidence events

$$
\varepsilon_{\text {LAS }}=\frac{1 \mathrm{X} \cdot 2 \mathrm{X} \cdot 3 \mathrm{X} \cdot 4 \mathrm{X}}{\text { Coincidence Events }}
$$

The recoil chamber efficiency is the ratio of the recoll $x$ - and $y$-plane coincidences to the coincidences of front and back scintillator counters

$$
\varepsilon_{\mathrm{R}}=\frac{\mathrm{X} \cdot \mathrm{Y}}{\text { Recoil Coincidences }} \text {. }
$$

The total efficiency is $\varepsilon_{\mathrm{LAS}{ }^{-} \varepsilon_{\mathrm{R}}}$ and the monitor was corrected for it. 


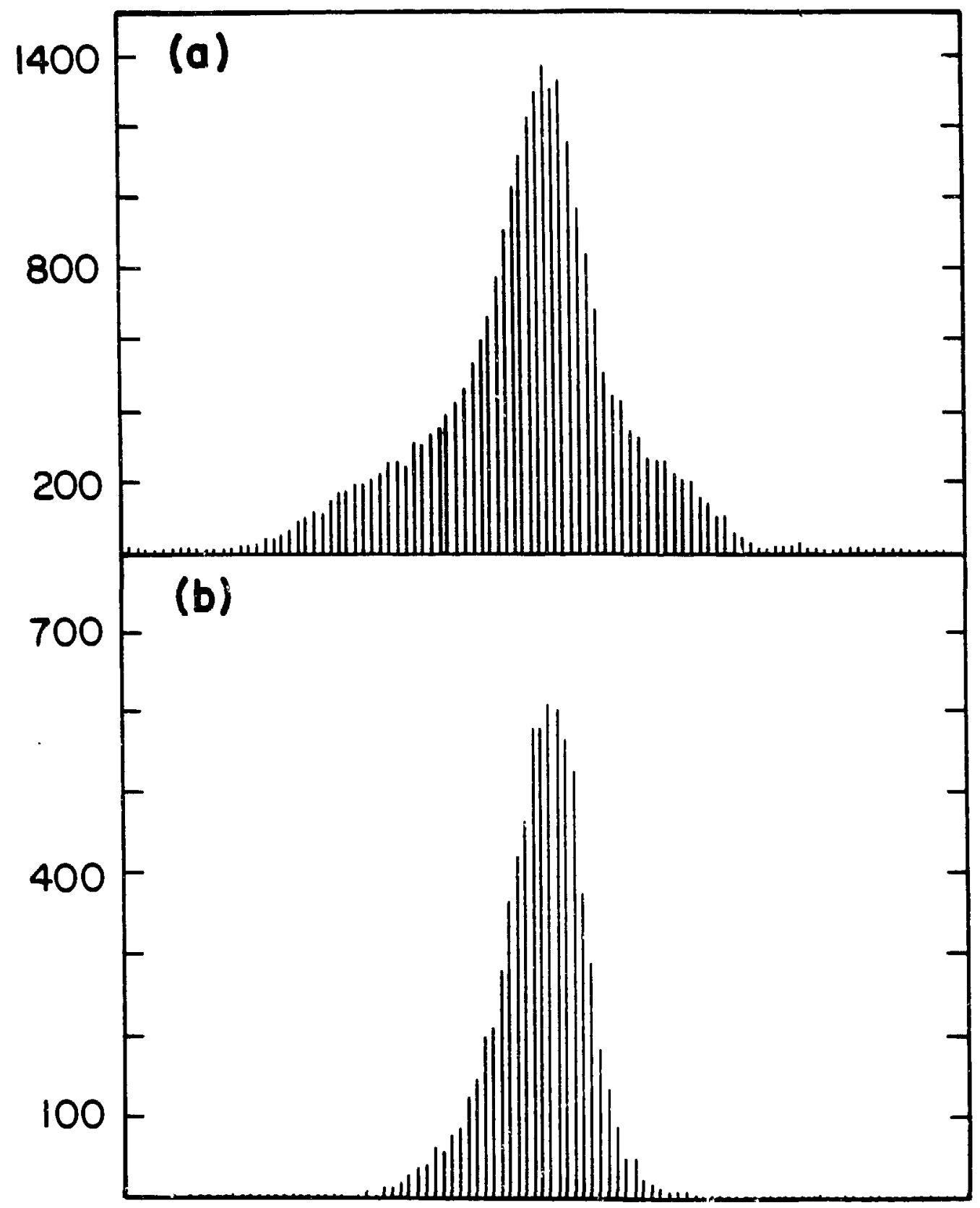

Figure 3.9. The LAS momentum spectrum. Each channel is $1 \mathrm{MeV} / \mathrm{c}$. (a) Spectrum without the cuts. (b) Spectrum with all the cuts applied to it. 
Table 3.1 A representative set of efficiencies for $471 \mathrm{MeV} / \mathrm{c}$ data.

\begin{tabular}{|c|c|c|c|c|c|}
\hline $\begin{array}{l}\theta_{\mathrm{cm}}^{\pi} \\
(\mathrm{Deg})\end{array}$ & $\begin{array}{c}\text { Target } \\
\text { Polarization } \\
\text { Direction }\end{array}$ & $\begin{array}{c}\varepsilon_{\text {LAS }} \\
(\%)\end{array}$ & $\begin{array}{l}\varepsilon_{\mathrm{R}} \\
(\%)\end{array}$ & $\begin{array}{l}\text { Dead } \\
\text { Time } \\
(\%)\end{array}$ & $\frac{\text { Monitor } \times \varepsilon_{\text {LAS }} \times \varepsilon_{R}}{\text { Dead TIme }}$ \\
\hline$\underset{" 1}{62.5}$ & $\begin{array}{l}\downarrow \\
\downarrow\end{array}$ & $\begin{array}{l}51.0 \\
49.7\end{array}$ & $\begin{array}{l}97.7 \\
95.2\end{array}$ & $\begin{array}{l}1.21 \\
1.20\end{array}$ & $\begin{array}{r}17.7 \\
8.8\end{array}$ \\
\hline $\begin{array}{l}72.1 \\
" 1\end{array}$ & $\begin{array}{l}t \\
\downarrow \\
-\end{array}$ & $\begin{array}{l}44.7 \\
45.9 \\
46.0\end{array}$ & $\begin{array}{l}96.5 \\
96.2 \\
90.6\end{array}$ & $\begin{array}{l}1.19 \\
1.17 \\
1.00\end{array}$ & $\begin{array}{r}7.9 \\
14.6 \\
25.7\end{array}$ \\
\hline${ }_{11}^{84.4}$ & $\uparrow$ & $\begin{array}{l}44.4 \\
45.3\end{array}$ & $\begin{array}{l}96.7 \\
95.1\end{array}$ & $\begin{array}{l}1.11 \\
1.10\end{array}$ & $\begin{array}{l}17.2 \\
18.7\end{array}$ \\
\hline $\begin{array}{c}94.2 \\
" 1 "\end{array}$ & $\begin{array}{l}\uparrow \\
+ \\
-\end{array}$ & $\begin{array}{l}44.3 \\
44.3 \\
46.0\end{array}$ & $\begin{array}{l}94.9 \\
94.1 \\
88.9\end{array}$ & $\begin{array}{l}1.10 \\
1.09 \\
1.09\end{array}$ & $\begin{array}{r}22.9 \\
23.0 \\
9.5\end{array}$ \\
\hline $\begin{array}{c}120.2 \\
" 1\end{array}$ & $\begin{array}{l}\uparrow \\
+ \\
-\end{array}$ & $\begin{array}{l}37.2 \\
38.3 \\
36.9\end{array}$ & $\begin{array}{l}46.5 \\
52.2 \\
34.2\end{array}$ & $\begin{array}{l}1.08 \\
1.08 \\
1.09\end{array}$ & $\begin{array}{r}18.2 \\
22.4 \\
7.9\end{array}$ \\
\hline $\begin{array}{c}134.3 \\
" 1\end{array}$ & $\begin{array}{l}t \\
\downarrow \\
-\end{array}$ & $\begin{array}{l}47.4 \\
47.8 \\
49.1\end{array}$ & $\begin{array}{l}52.4 \\
52.9 \\
58.0\end{array}$ & $\begin{array}{l}1.08 \\
1.08 \\
1.08\end{array}$ & $\begin{array}{l}43.1 \\
11.3 \\
36.0\end{array}$ \\
\hline $\begin{array}{c}154.2 \\
" 1 \\
"\end{array}$ & $\begin{array}{l}\uparrow \\
+ \\
-\end{array}$ & $\begin{array}{l}53.3 \\
53.2 \\
51.0\end{array}$ & $\begin{array}{l}82.5 \\
84.7 \\
74.7\end{array}$ & $\begin{array}{l}1.09 \\
1.09 \\
1.10\end{array}$ & $\begin{array}{l}14.8 \\
54.9 \\
21.1\end{array}$ \\
\hline
\end{tabular}




\subsection{Target Polarization Analysis}

To understand the operational principles of NMR, consider a proton, whose only degrees of freedom are the spin states, under the Influence of a large magnetic field $B_{0}$ pointing in the $z$-direction, and constant in time, and a small oscillating field bcoswt, pointing in the $x$-direction. The Hamiltonian of the system is

$$
\mathrm{H}=-\vec{\mu} \mathrm{P} \cdot \overrightarrow{\mathrm{B}}
$$

where $\vec{\mu}_{\mathrm{P}}=g \frac{\mathrm{eh}}{2 \mathrm{~m}_{\mathrm{P}} \mathrm{c}} \overrightarrow{\mathrm{S}}=\mathrm{g} \frac{\mathrm{eh}}{4 \mathrm{~m}_{\mathrm{P}} \mathrm{c}} \stackrel{\vec{\sigma}}{ }$ and $\overrightarrow{\mathrm{B}}=\mathrm{b} \cos \omega \hat{\mathrm{u}}_{\mathrm{x}}+\mathrm{B}_{\mathrm{O}} \hat{\mathrm{u}}_{\mathrm{z}}$. The Schrodinger equation

$$
H \Psi=1 h \frac{\partial \Psi}{\partial t}
$$

now reads

$$
\text { ih } \frac{d}{d t}\left(\begin{array}{l}
\psi_{1}(t) \\
\psi_{2}(t)
\end{array}\right)=g \frac{e h}{4 m_{P} c}\left(\begin{array}{c}
B_{0} b \cos \omega t \\
b \cos \omega t-B_{0}
\end{array}\right)\left(\begin{array}{l}
\psi_{1}(t) \\
\psi_{2}(t)
\end{array}\right)
$$

where $\Psi=\left(\begin{array}{l}\psi_{1}(t) \\ \psi_{2}(t)\end{array}\right)$. Defining

$$
\omega_{0}=g \frac{e B_{0}}{4 m_{p} c}, \quad \omega_{o}^{-}=g \frac{e b}{4 m_{p} c}
$$

we see that if at $t=0$ the spin points in the positive $z$-direction, then at some later time $t$, the probability that the spin points in the negative $z$-direction is

where

$$
P(++-)=\frac{\omega_{0}^{-2}}{2 \Omega^{2}}(1-\cos \Omega t)
$$

$$
\Omega^{2}=\left(2 \omega_{0}-\omega\right)^{2}+\omega_{0}^{-2} .
$$

When the frequency of the oscillating field is "tuned" to match $2 \omega_{0}$ ( ( $_{0}$ being the nuclear L - ror frequency in the PTM field), then $\Omega+\omega_{0}^{-}$and the spin flip probability, that is, the probability of the RF field causing a spin flip reaches its maximum. Since the energy of the "up" state is different from that of the "down" state, such an energy 
difference, absorbed from the external fleld, signals the resonance frequency. Because this process depolarizes the target, it is necessary to limic the RF power used so as to reduce the amount of deporarization to an insignificant level.

To obtain an absolute target polarization the system is calibrated at thermal equilibrium (microwaves off) where the polarization is known from Boltzman statistics. A relatively high temperature (about $1{ }^{\circ} \mathrm{K}$ ) was chosen for this calibration so that the spin lattice relaxation time is not excessively long and equilibrium can be achieved in a reasonable length of time. In thermal equilibrium at temperature $\mathrm{T}$, the polarization is given by

$$
\mathrm{P}_{0}=\tanh \frac{\mu_{P^{B}}}{K_{B}^{T}} .
$$

At equilibrium (i.e. when the NMR iignal has stcpped growing or decaying) the polarization is known if the temperature (and magnetic field) is known. The area of the absorption signal, $A$, is proportional to the nuclear polarization, P. By comparing the area of the signal from dynamically polarized nuclei to the area $A_{0}$ of the thermal equilibrium signal of known polarization $P_{0}$, the polarization $P$ is given by

$$
\frac{\mathrm{P}}{\mathrm{P}_{0}}=\frac{\mathrm{A}}{\mathrm{A}_{0}} .
$$

This calibration method involves no assumptions about the size or the shape of the target or the mechanism of polarization. The most serious Imitation of this method is poor signal-to-noise ratio at theriual equilibrium, resulting from imprecise measurement of $A_{0} \cdot$ This problem 
is partially overcome by using signal averaging and by measuring the background with the magnetic field slightly shifted.

Measurements of the peak area for the enhanced signal was not a major problem because the signal was much larger than the background. The accuracy of the thermal equilibrium measurement determines the accuracy of the target polarization. For a target temperature of $l^{\circ} \mathrm{K}$ in a 25 KGauss magnetic field, the natural polarization is only $0.26 \%$. In order to measure this small signal, reducing random noise sad enhancing the signal area, 256 measurements were accumulated in the signal averager. To subtract the random noise the PTM field was varied, moving the nuclear resonance frequency outside the frequency range swept by the system. Subtracting 256 measurements of the random noise, the remaining area was used in the calibration. Figure 3.10 shows a typical thermal equilibrium spectrum. The background was fitted with a second order polynomial (dotted line in figure). The calibration constant remain unchanged for each target filling. In this experiment the target polarization was near $80 \%$, and was measured with a relative accuracy of typically $(\Delta \mathrm{P} / \mathrm{P}=3 \%$. A typical target polarization at each momentum is given in table 3.2 to show the stability of the polarization as a function of time. 
Table 3.2 Target polarizarion as a function of time.

\begin{tabular}{|c|c|c|c|c|}
\hline $\begin{array}{c}\text { Beam } \\
\text { Momentum } \\
(\mathrm{MeV} / \mathrm{c})\end{array}$ & $\begin{array}{c}\text { Beam } \\
\text { Polarity }\end{array}$ & $\begin{array}{c}\theta_{\mathrm{cm}}^{\pi} \\
\text { (Degree ) }\end{array}$ & $\begin{array}{c}\text { Target } \\
\text { Polarization } \\
\text { Direction }\end{array}$ & $\begin{array}{c}\text { Target } \\
\text { Polarization } \\
(\%)\end{array}$ \\
\hline 471 & $\pi^{+}$ & 84.3 & $\uparrow$ & $\begin{array}{l}80.7 \\
80.3\end{array}$ \\
\hline$"$ & $\pi^{-}$ & 154.2 & $\begin{array}{l}t \\
\downarrow\end{array}$ & $\begin{array}{l}80.2 \\
81.7\end{array}$ \\
\hline $\begin{array}{c}547 \\
" 1\end{array}$ & $\pi^{+}$ & 135.0 & $\uparrow$ & $\begin{array}{l}81.4 \\
82.8\end{array}$ \\
\hline " & $\pi^{-}$ & $\frac{95.7}{11}$ & $\uparrow$ & $\begin{array}{l}81.7 \\
81.4\end{array}$ \\
\hline $\begin{array}{c}625 \\
" 1\end{array}$ & $\pi^{+}$ & 6.5.0 & $\uparrow$ & $\begin{array}{l}80.4 \\
81.1\end{array}$ \\
\hline$" 1 "$ & $\pi^{-}$ & 81.0 & $\downarrow$ & $\begin{array}{l}81.5 \\
79.8\end{array}$ \\
\hline $\begin{array}{c}687 \\
1 "\end{array}$ & $\pi^{+}$ & 115.0 & $\uparrow$ & $\begin{array}{l}80.7 \\
80.6\end{array}$ \\
\hline " & $\pi^{-}$ & $\begin{array}{l}65.0 \\
11\end{array}$ & $\downarrow$ & $\begin{array}{l}78.3 \\
81.0\end{array}$ \\
\hline
\end{tabular}




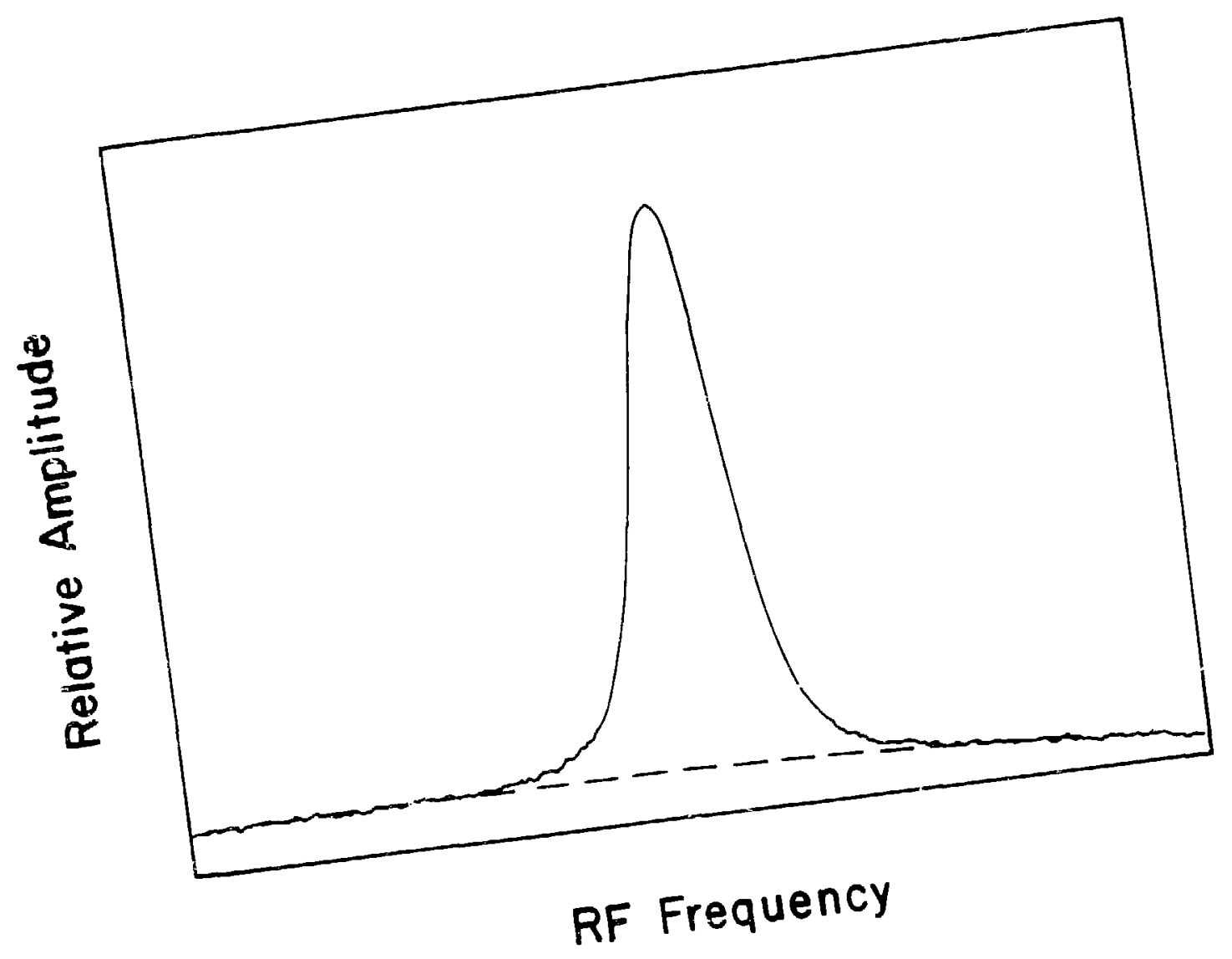
Figure 3.10 . Typical thermal equilibrium spectrum produced by the NMR
system. The dotted curve represents a quadratic fit to the background. 


\subsection{The Background}

The largest error source was a background of coincidences resulting from quasi-elastic and non-elastic reactions of pions with the carbon and oxygen of the propanediol, ${ }^{3}$ He of the helfum bath, ${ }^{4}$ He and materials of the target cryostat. The presence of nuclei other than protons in the target is not a serious drawback for low-energy scattering. Because of the existence of an iricernal Fermi energy for the nucleons inside these nuclei, scattering from them occurs with an energy different from scattering by the tree (and polarized) protons of the target and the two types of events are easily discriminated. On the contrary, in high-energy physics this discrimination, based on differences in energy much smaller than of the beam, is far less efficient and leads to a large background to be subtracted by comparison with a "dummy" target. The outgoing pioa from the quasi-elastic channel does not have well-defined momentum as a pion from elastic scattering. Quasi-elastic scattered pions have a momentum range whose limits are a function of angle for a given beall momentum. The upper limit represents the scattering of a pion which supplied the minimum amount of energy $(\sim 8.5 \mathrm{MeV})$ required to free a nucleon. The background angular dependence at each momentum was measured explicitly in separate runs with propanediol replaced by carbon (hydrogen-free dumny target) with approximately the same number of carbon nuclei. The carbon and oxygen nuclei in the propanediol target have $J=0$ and are not polarized. This background is treated as being independent of target polarization and has been subtracted from all yields. 
Accidentals are the result of uncorrelated events accidentally satisfying coincidence requirements as well as software cuts. By making the software cuts tighter, the background is reduced but some good events are lost too. While this is not acceptable for determining cross sections, it is for measuring the analyzing power.

To determine the ratio of carbon in the dummy target to the propanediol in the polarized target, the flat distribution in the outer channels below and above the the elastic peak were used. Comparing this to the background that was explicitly measured, the amount of carbon in the dummy target was obtained. Cuts placed on the LAS momentum spectrum defined that part of the spectrum considered piure background (regions $A$ and $C$ in $f$ tgure 3.11 ), and that part which was a mixture of background and good events (region B In figure 3.11). Only the LAS TOF cuts were used for this purpose. This ratio varied every time the target was refilled. The typical variation was of the order of $\pm 10 \%$. As an example for $471 \mathrm{MeV} / \mathrm{c} \pi^{+}$

$$
\frac{\mathrm{m}_{\text {carbon }}}{\mathrm{m}_{\mathrm{C}_{3} \mathrm{H}_{802}}}=0.94 \pm 0.10
$$

Once normalized, the background in region $B$ was subtracted. Tnis region was then split into two bins and the asymmetry was calculated for each bin separately.

In order to check the internal consistancy of the data, cuts on the LAS TOF, cuts on the TOF between individual recoll counters and the LAS front counter S1 (TB), and on the sum (TS), and the difference (TD) of the signals in these counters were first used. This incroduced the 


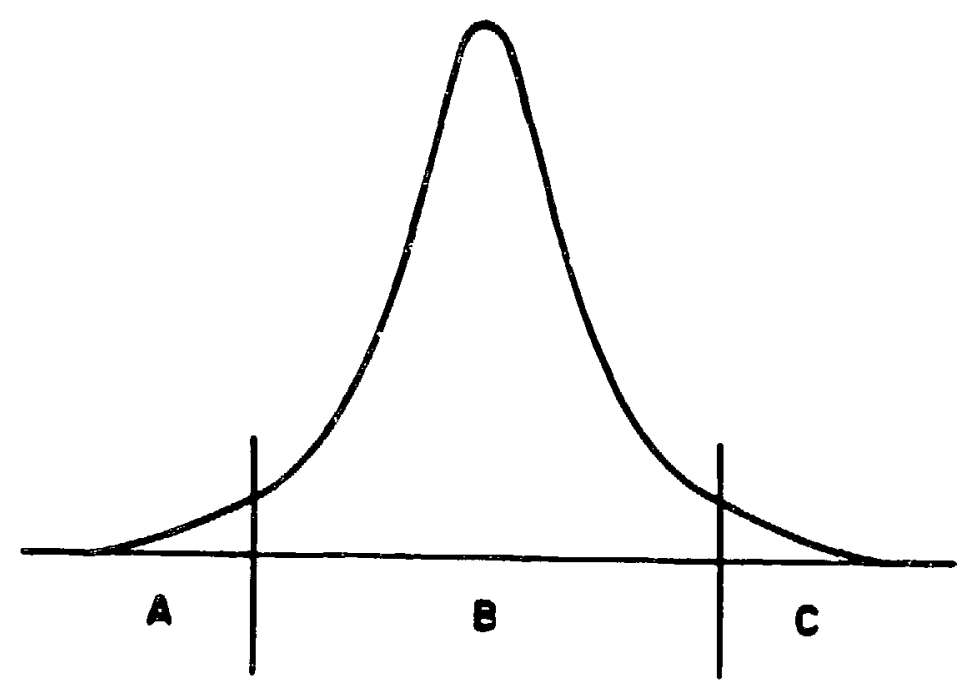

Figure 3.11. Regions of the LAS momentum spectrum used to normalize background subtraction.

ininimum chamber inefficiencies into the calculation of the analyzing power, the corrected monitor is, in this case

$$
\frac{\text { Monitor } \times \varepsilon_{\text {LAS }}}{\text { Dead Time }}
$$

The LAS particle's momentum spectrum was then divided into four bins and the analyzing power was calculated for each bin separately. The results are given in tha first 1 ine of table $3.4(a)$. Then, the recoil $\mathrm{X}$-plane wide open cuts were added to the above ToF cuts, and the results are given on the second line of the table. The corrected monitor is, in this case

$$
\frac{\text { Monitor } \times \varepsilon_{\text {LAS }} \times \varepsilon_{R}^{X}}{\text { Dead T1me }},
$$

where $E_{R}^{X}$ is the recoil $X$-plane efficiency. Line 3 in the table presents the same thing as the second line, except that tighter cuts 
are applied to the recoll X-plane. On line 4 the results of adding wide open recoll Y-plane cuts to the TOF cuts of the first line are given. The corrected monitor is, in this case

$$
\frac{\text { Monitor } \times \varepsilon_{\text {LAS }} \times \varepsilon_{\mathrm{R}}^{\mathrm{Y}}}{\text { Dead Time }}
$$

where the $E_{R}^{Y}$ is the recoil $Y$-plane efficiency. Line 5 is the same as line 4, except that cuts are tighter. Line 6 of the table gives the results for the coincidence of $T O F \cdot T B \cdot T S \cdot T D \cdot X \cdot Y$. The corrected monitor is, in this case

$$
\frac{\text { Monitor } \times \varepsilon_{\text {LAS }^{\times}}{ }^{\mathrm{E}} \mathrm{R}}{\text { Dead Time }}
$$

where $\varepsilon_{\mathrm{R}}$ is defined previously. Line 7 presents the results of adding wide open TGTX to line 6 . Line 8 is the same as 1 ine 7 , except that tighter cuts are applied to TGTX. Line 9 presents the results of adding wide open TGTY to line 6 . The ${ }^{\varepsilon}$ LAS was corrected to take account of the efficiency of the LAS first two Y-planes

$$
E_{\text {LAS }}^{-}=\frac{1 \mathrm{X} \cdot 2 \mathrm{X} \cdot 3 \mathrm{X} \cdot 4 \mathrm{X} \cdot 1 \mathrm{Y} \cdot 2 \mathrm{Y}}{\text { Coincidence Event } \mathrm{s}}
$$

and the corrected monitor is, in this case

$$
\frac{\text { Monitor } \times E_{\text {LAS }}^{\prime}{ }^{\times E} \mathrm{R}}{\text { Dead Time }}
$$

Line 10 gives the results of the coincidence between $\mathrm{TOF} \cdot \mathrm{TB} \cdot \mathrm{TS} \cdot \mathrm{TD} \cdot \mathrm{X} \cdot \mathrm{Y} \cdot \mathrm{TGTX} \cdot \mathrm{TGTY}$. Finally, on line 11 , the results of the addition of HANG cuts to line 10 are given. The $\varepsilon_{\text {LAS }}$ was corrected to include the efficiency of all the 8 planes of the LAS

$$
E_{\text {LAS }}^{\prime \prime}=\frac{1 X \cdot 2 X \cdot 3 X \cdot 4 X \cdot 1 Y \cdot 2 Y \cdot 3 Y \cdot 4 Y}{\text { Coincidence }}
$$


and the corrected monitor is, in this case

$$
\frac{\text { Monitor } \times E_{\text {LAS }}^{\prime \prime} \varepsilon_{R}}{\text { Dead Time }}
$$

The internal consistancy of the data is obvious from this table. Table $3.4(b)$ is similar to table $3.4(a)$ except that it shows the results when the background was not measured, but was estimated. As another check the difference of polarization up and down yields, Y†-Y $\downarrow$, normalized to the polarization up run and corrected for chamber efficiency and dead time, and also $Y \uparrow+Y \downarrow-2 B$, were plotted. If the background is indepenaent of the target polarization direction, these quantities must contain no background and when histogrammed both have the same shape (see figure 3.12). In the difference, for example, off peak yield almost completly cancels out, and there is less than one event per bin, this confirms that normalization was done correctly (and that the background is indeperdent of the target polarization).

At $471 \mathrm{MeV} / \mathrm{c} \pi^{+} \mathrm{p}$, background was measured at only $\theta_{\mathrm{cm}}=62.5^{\circ}$, $84.3^{\circ}, 107.2^{\circ}$, and $154.5^{\circ}$. To estimate the background at $\theta_{\mathrm{cm}}=72.1^{\circ}$, $95.8^{\circ}, 120.3^{\circ}$, and $134.3^{\circ}$ the normalized background was plotted (same software cuts applied for each momentum) versus the center of mass angle. Fitting a curve to the measured background, the unmeasured background were estimated easily. See figure 3.13. 


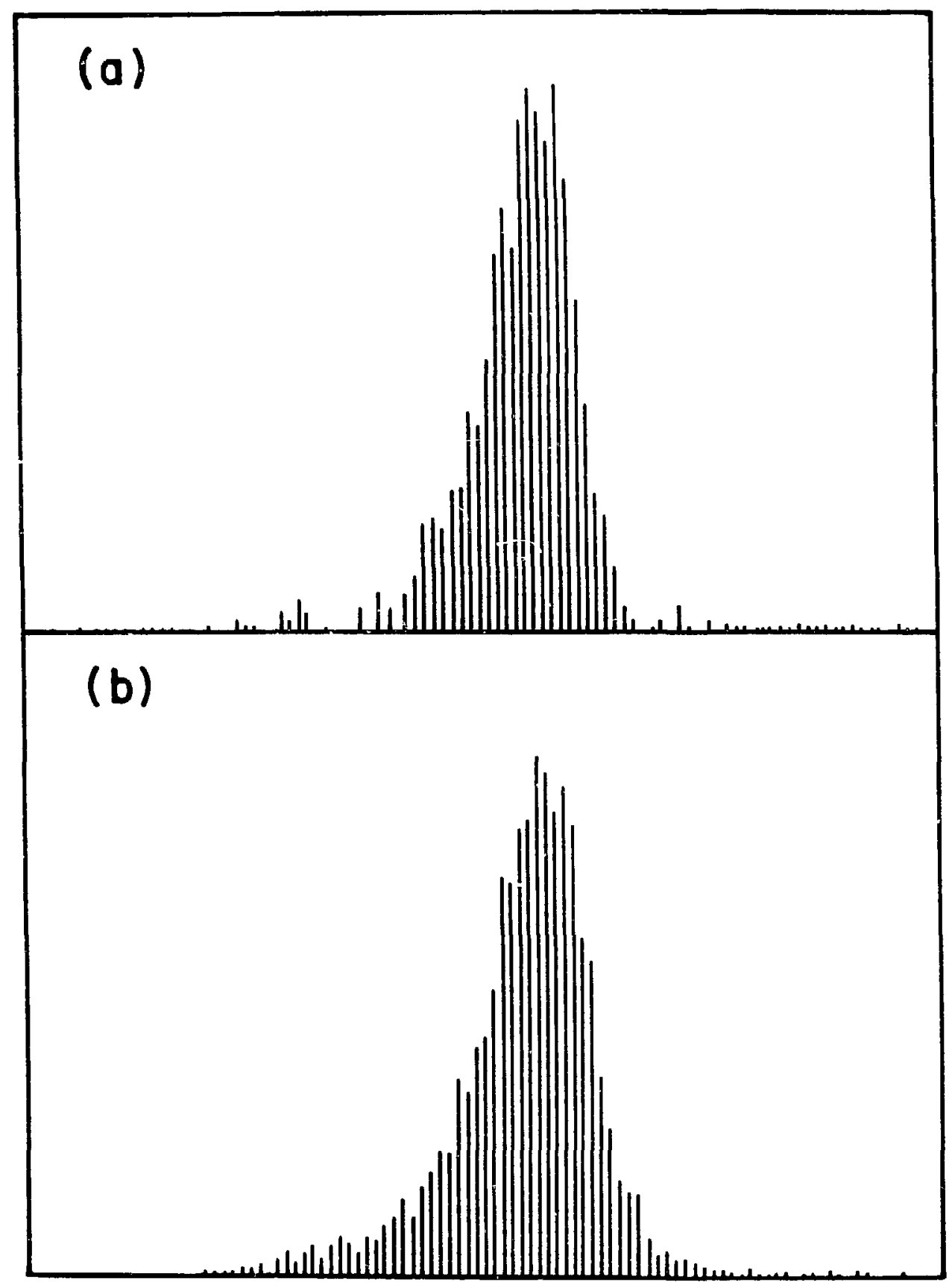

Figure 3.12. (a) The difference between polarization up and
polarization down yields, Yt-Yt, for $625 \mathrm{MeV} / \mathrm{c} \pi^{-}$at $\theta_{\mathrm{cm}}=81^{\circ}$. (b) $\mathrm{Y}++\mathrm{Y} \downarrow-2 \mathrm{~B}$. 
Table 3.3(a) Ratio of background to signal for $471 \mathrm{MeV} / \mathrm{c} \pi^{+}$data after applying the cuts.

\begin{tabular}{cccc}
\hline$\theta_{\mathrm{cm}}^{\pi}$ & $\begin{array}{c}\text { PTM } \\
\text { Field } \\
\text { Direction }\end{array}$ & $\frac{\text { Background }}{\text { Polarization Up }}$ & $\frac{\text { Background }}{\text { Polarization Down }}$ \\
\hline degree $)$ & & $(\%)$ & $(\%)$ \\
\hline $62.5^{*}$ & $\uparrow$ & 2.4 & 1.9 \\
$72.1^{*}$ & $\uparrow$ & 2.4 & 1.8 \\
$94.3^{\star}$ & $\uparrow$ & 2.8 & 1.7 \\
$107.2^{*}$ & $\uparrow$ & 3.0 & 20.6 \\
$120.3^{*}$ & $\downarrow$ & 24.0 & 27.0 \\
$134.3^{*}$ & $\downarrow$ & 22.5 & 22.5 \\
$154.4^{*}$ & $\downarrow$ & 16.6 & 15.6 \\
\hline
\end{tabular}

* Background was not measured, it was estimated.

Table 3.3(b) Ratio of background to signal for $547 \mathrm{MeV} / \mathrm{c} \pi^{+}$data after applying the cuts.

\begin{tabular}{|c|c|c|c|}
\hline$\theta^{\pi}$ & $\begin{array}{l}\text { PTM } \\
\text { Field }\end{array}$ & Background & Background \\
\hline & Direction & Polarization Up & Polarization Down \\
\hline (degree) & & $(\%)$ & $(\%)$ \\
\hline $\begin{array}{l}60.0 \\
70.0\end{array}$ & $\uparrow$ & $\begin{array}{l}0.2 \\
1.9\end{array}$ & $\begin{array}{l}0.2 \\
2.0\end{array}$ \\
\hline 80.0 & $\uparrow$ & 0.0 & 0.0 \\
\hline 90.0 & $\uparrow$ & 4.2 & 4.9 \\
\hline $105 \cdot 0^{*}$ & $\uparrow$ & 37.7 & 36.3 \\
\hline 120.0 & $\downarrow$ & 12.0 & 20.7 \\
\hline 135.0 & $\downarrow$ & 0.5 & 0.9 \\
\hline
\end{tabular}

* Background was not measured, it was estimated. 
Table 3.3(c) Ratio of background to signal for $625 \mathrm{MeV} / \mathrm{c} \pi^{+}$data after applying the cuts.

\begin{tabular}{cccc}
\hline$\theta_{\mathrm{cm}}^{\pi}$ & $\begin{array}{c}\text { PTM } \\
\text { Field } \\
\text { Direction }\end{array}$ & $\begin{array}{c}\text { Background } \\
\text { Polarization UP }\end{array}$ & $\begin{array}{c}\text { Background } \\
\text { Polarization Down }\end{array}$ \\
\hline 50.0 & & $(\%)$ & $(\%)$ \\
65.0 & $\uparrow$ & & 28.0 \\
80.0 & + & 6.3 & 5.8 \\
108.0 & $\uparrow$ & 15.8 & 63.0 \\
120.0 & + & 33.4 & 63.9 \\
150.0 & + & 30.9 & 44.0 \\
\hline
\end{tabular}

Table 3.3(d) Ratio of background to signal for $687 \mathrm{MeV} / \mathrm{c}^{+}$data after applying the cuts.

\begin{tabular}{rccc}
\hline$\theta_{\mathrm{cm}}^{\pi}$ & $\begin{array}{c}\text { PTM } \\
\text { Field } \\
\text { Direction }\end{array}$ & $\frac{\text { Background }}{\text { Polarization Up }}$ & $\frac{\text { Background }}{\text { Polarization Down }}$ \\
\hline (degree) & & $(\%)$ & $(\%)$ \\
\hline 60.0 & $\uparrow$ & 24.4 & 22.5 \\
80.0 & $\uparrow$ & 15.5 & 12.1 \\
100.0 & $\uparrow$ & 0.0 & 60.9 \\
115.0 & + & 70.3 & 30.9 \\
135.0 & + & 29.2 & 7.8 \\
155.0 & + & 5.5 & \\
\hline
\end{tabular}


Table 3.3(e) Ratio of background to signal for $471 \mathrm{MeV} / \mathrm{c}^{-}$data after applying the cuts.

\begin{tabular}{cccc}
\hline$\theta_{\text {cm }}^{\pi}$ & $\begin{array}{c}\text { PTM } \\
\text { Field } \\
\text { Direction }\end{array}$ & $\frac{\text { Backgrcund }}{\text { Polarization Up }}$ & $\frac{\text { Background }}{\text { Polarization Down }}$ \\
\hline (degree) & & $(\%)$ & $(\%)$ \\
\hline 72.5 & + & 0.6 & 3.0 \\
$84.4^{*}$ & + & 0.8 & 3.2 \\
$94.4^{*}$ & + & 2.0 & 4.9 \\
120.2 & + & 4.0 & 6.1 \\
$134.3^{*}$ & $\uparrow$ & 30.6 & 12.2 \\
154.2 & $\uparrow$ & 26.1 & 11.9 \\
\hline
\end{tabular}

* Background was not measured, it was estimated.

Table 3.3(f) Ratio of background to signal for $547 \mathrm{MeV} / \mathrm{c}^{-}$data after applying the cuts.

\begin{tabular}{cccc}
\hline$\theta_{\mathrm{cm}}^{\pi}$ & $\begin{array}{c}\text { PTM } \\
\text { Field } \\
\text { Direction }\end{array}$ & $\begin{array}{c}\text { Background } \\
\text { Polarization Up }\end{array}$ & $\frac{\text { Background }}{\text { Polarization Down }}$ \\
\hline & & $(\%)$ & $(\%)$ \\
\hline 53.0 & $\uparrow$ & & 12.5 \\
60.0 & $\uparrow$ & 3.4 & 15.5 \\
72.5 & $\uparrow$ & 5.3 & 17.8 \\
84.0 & $\uparrow$ & 6.2 & 30.8 \\
95.7 & $\uparrow$ & 18.3 & 17.2 \\
106.9 & $\uparrow$ & 27.5 & 69.1 \\
120.0 & $\uparrow *$ & 92.6 & 0.0 \\
134.3 & $\uparrow$ & 0.0 & 1.9 \\
150.0 & $\uparrow$ & 2.7 & \\
\hline
\end{tabular}

* Background was not measured, it was estimated.

**Single arm measurement. 
Table $3.3(\mathrm{~g})$ Ratio of background to signal for $625 \mathrm{MeV} / \mathrm{c}^{-}$data after applying the cuts.

\begin{tabular}{|c|c|c|c|}
\hline \multirow{2}{*}{$\theta_{\mathrm{cm}}^{\pi}$} & \multirow{3}{*}{$\begin{array}{c}\text { PTM } \\
\text { Field } \\
\text { Direction }\end{array}$} & Background & Background \\
\hline & & Polarization Up & Polarization Down \\
\hline (degree) & & $(\%)$ & $(\%)$ \\
\hline 51.0 & $\uparrow$ & 6.1 & 23.2 \\
\hline 65.7 & $\uparrow$ & 4.4 & 16.9 \\
\hline 81.0 & $\uparrow$ & 17.0 & 40.8 \\
\hline 109.3 & $\uparrow$ & 52.3 & 33.7 \\
\hline 140.0 & $\uparrow$ & 11.8 & 9.8 \\
\hline
\end{tabular}

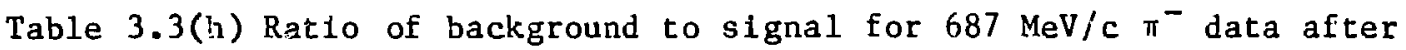
applying the cuts.

\begin{tabular}{|c|c|c|c|}
\hline$\theta_{\mathrm{cm}}^{\pi}$ & $\begin{array}{c}\text { PTM } \\
\text { Field } \\
\text { Direction }\end{array}$ & $\frac{\text { Background }}{\text { Polarization UP }}$ & $\frac{\text { Background }}{\text { Polarization Down }}$ \\
\hline (degree) & & (\%) & $(\%)$ \\
\hline 50.0 & $t$ & 9.0 & 21.8 \\
\hline 65.0 & $\uparrow$ & 10.2 & 3.3 \\
\hline 80.0 & $\uparrow$ & 18.2 & 4.7 \\
\hline 110.0 & $\uparrow$ & 11.9 & 9.4 \\
\hline 140.0 & $\uparrow$ & 4.1 & 6.5 \\
\hline 160.0 & $\uparrow$ & 2.4 & 3.1 \\
\hline
\end{tabular}




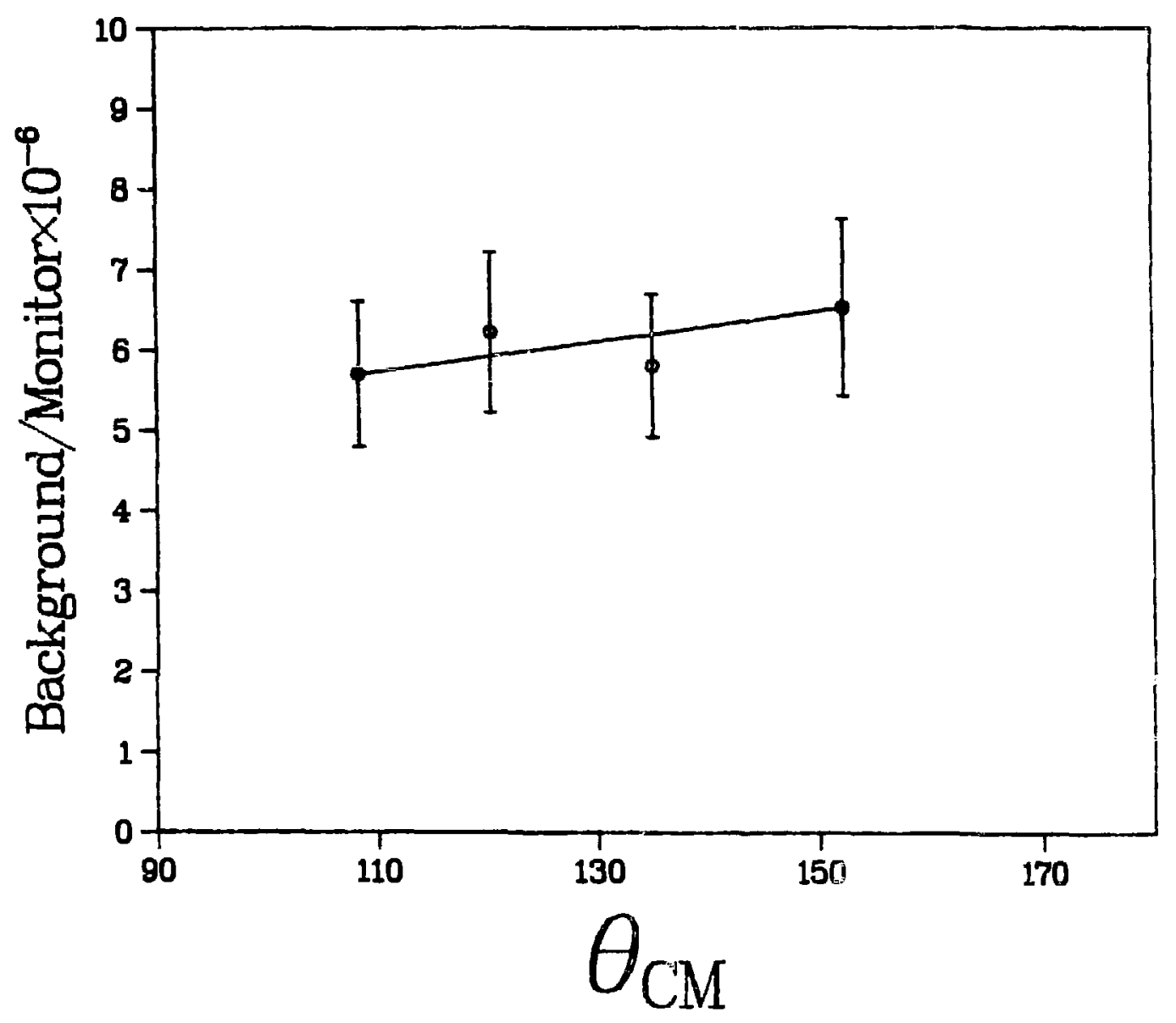

Figure 3.13. Normalized background vs $\theta_{\mathrm{cm}}$.

(•) Background was measuerej.

(o) Background was estimated. 


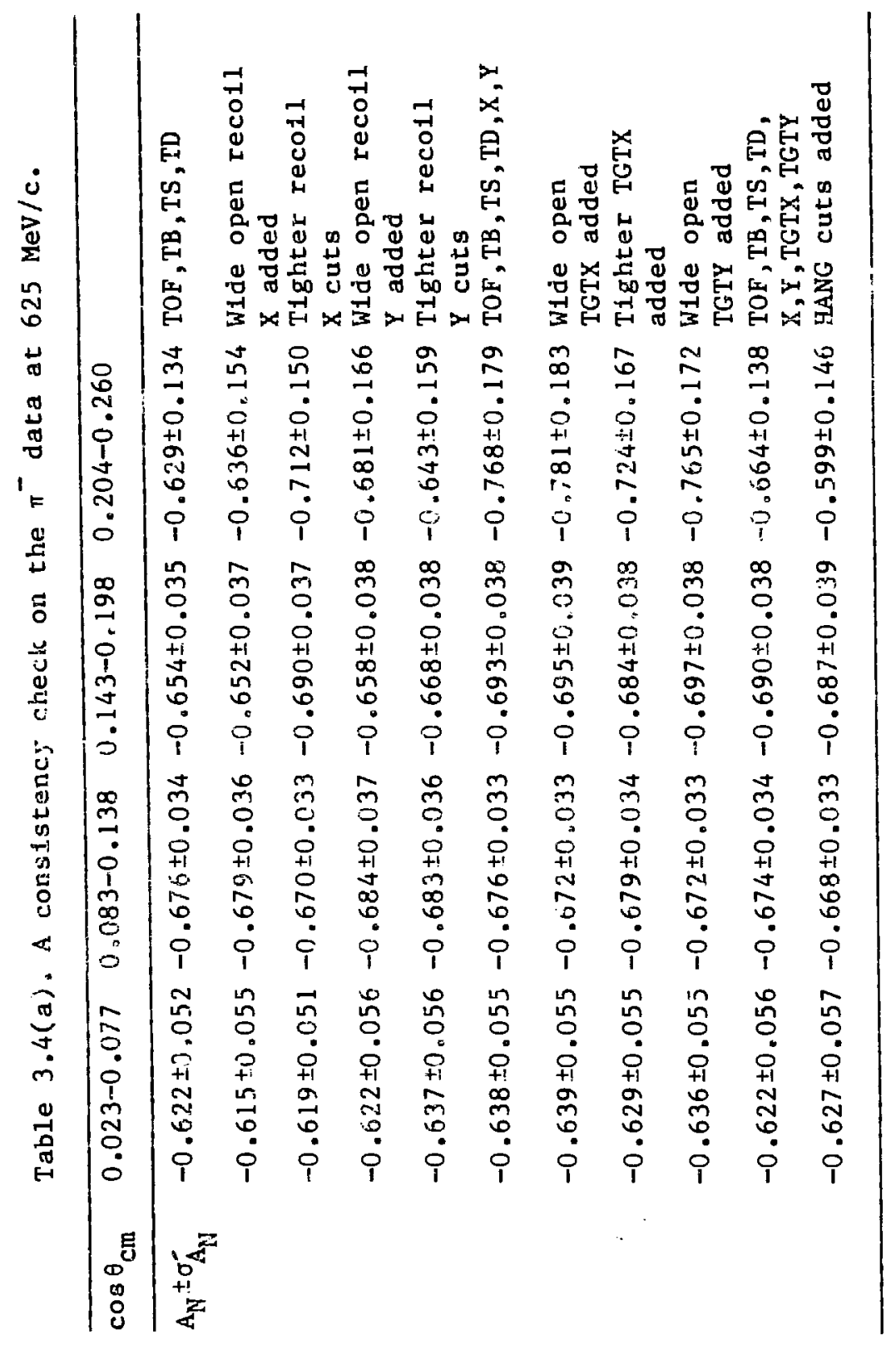


Table $3.4(\mathrm{~b})$. A consistancy check on the $\pi^{+}$data at $471 \mathrm{MeV} / \mathrm{c}$.

\begin{tabular}{|c|c|c|c|}
\hline $\cos \theta_{\mathrm{cm}}$ & $0.226-0.308$ & $0.308-0.391$ & \\
\hline$A_{N} \pm \sigma_{A_{N}}^{-}$ & $\begin{array}{l}0.176 \pm 0.027 \\
0.174 \pm 0.028 \\
0.194 \pm 0.029 \\
0.180 \pm 0.028 \\
0.190 \pm 0.029 \\
0.194 \pm 0.031 \\
0.193 \pm 0.031 \\
0.206 \pm 0.032 \\
0.210 \pm 0.032 \\
0.209 \pm 0.032 \\
0.219 \pm 0.032\end{array}$ & $\begin{array}{l}0.167 \pm 0.024 \\
0.164 \pm 0.024 \\
0.174 \pm 0.025 \\
0.167 \pm 0.024 \\
0.165 \pm 0.025 \\
0.169 \pm 0.026 \\
0.169 \pm 0.026 \\
0.164 \pm 0.027 \\
0.167 \pm 0.027 \\
0.166 \pm 0.027 \\
0.167 \pm 0.027\end{array}$ & 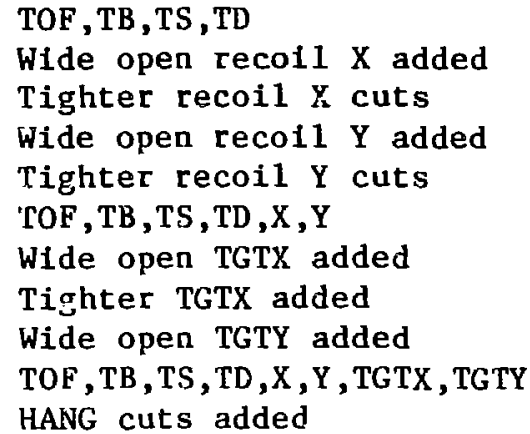 \\
\hline
\end{tabular}




\subsection{Statistical Uncertainty}

Suppose we want to determine a quantity $x$ which is a function of at least two other variables $u$ ard $v$ which are actually measured

$$
x=E(u, v, \ldots),
$$

we will assume that the most probable value for $x$ is given by

$$
\bar{x}=f(\bar{u}, \bar{v}, \ldots)
$$

The uncertainty in the resulting value for $x$ can be found by considering the spread of the values of $x$ resulting from combining the individual measurements $u_{i}, v_{i}, \ldots$ into individual results $x_{i}$

$$
x_{i}=E\left(u_{i}, v_{i}, \ldots\right) \text {. }
$$

In the limit of an infinite number of measurements, the mean of this distribution will coincide with the average $\bar{x}$ given in eq. (3.1). The variance is defined as the limit of the average of the squares of the deviations from the mean

$$
\sigma_{x}^{2}=\lim _{N \rightarrow \infty}\left[\frac{1}{N} \sum\left(x_{i}-\bar{x}\right)^{2}\right]
$$

and the standard deviation is the square root of the variance. Thus, the standard deviation is the root mean square of the deviations. We can express the deviations $x_{i}-\bar{x}$ in terms of the deviations $u_{i}-\bar{u}$, $v_{i}-\bar{v}, \ldots$ of the observed parameters

$$
x_{i}-\bar{x} \simeq\left(u_{i}-\bar{u}\right)\left(\frac{\partial x}{\partial u}\right)+\left(v_{i}-\bar{v}\right)\left(\frac{\partial x}{\partial v}\right)+\cdots,
$$

where each of the partial derivatives is evaluated with all other vartables fixed at their mean values. Combining eqs. (3.2) and (3.3) 
we can express the variance $\sigma_{x}^{2}$ for $x$ in terms of the variances $\sigma_{u}^{2}$, $\sigma_{v}^{2}, \ldots$ for the variables $u, v, \ldots$ which were actually measured

$$
\begin{aligned}
\sigma_{x}^{2} & \simeq \lim _{N \rightarrow \infty} \frac{1}{N} \int\left[\left(u_{i}-\bar{u}\right)\left(\frac{\partial x}{\partial u}\right)+\left(v_{i}-\bar{v}\right)\left(\frac{\partial x}{\partial v}\right)+\cdots\right]^{2}, \\
& \simeq \lim _{N \rightarrow \infty} \frac{1}{N} \int\left[\left(u_{i}-\bar{u}\right)^{2}\left(\frac{\partial x}{\partial u}\right)^{2}+\left(v_{i}-\bar{v}\right)^{2}\left(\frac{\partial x}{\partial v}\right)^{2}+2\left(u_{i}-\bar{u}\right)\left(v_{i}-\bar{v}\right)\left(\frac{\partial x}{\partial u}\right)\left(\frac{\partial x}{\partial v}\right)+\cdots\right] .
\end{aligned}
$$

The first two terms in this equation can be expressed in terms of the variances $\sigma_{u}^{2}$ and $\sigma_{v}^{2}$

$$
\begin{aligned}
& \sigma_{u}^{2}=\lim _{N \rightarrow \infty} \frac{1}{N} \sum\left(u_{i}-\bar{u}\right)^{2}, \\
& \sigma_{v}^{2}=\lim _{N \rightarrow \infty} \frac{1}{N} \sum\left(v_{i}-\bar{v}\right)^{2},
\end{aligned}
$$

and the third term can be expressed in terms of the covariance belween the rariables $u$ and $v$, defined by

$$
\sigma_{u v}^{2}=\lim _{N \rightarrow \infty} \frac{1}{N}\left[\left[\left(u_{i}-\bar{u}\right)\left(v_{i}-\bar{v}\right)\right]\right. \text {. }
$$

witn these definitions, the approximation for the standard deviation can he expressed as

$$
\sigma_{x}^{2} \simeq \sigma_{u}^{2}\left(\frac{\partial x}{\partial u}\right)^{2}+\sigma_{v}^{2}\left(\frac{\partial x}{\partial v}\right)^{2}+2 \sigma_{u v}\left(\frac{\partial x}{\partial u}\right)\left(\frac{\partial x}{\partial v}\right)+\cdots
$$

The first two terms are averages of squares of deviations, which will presumably dominate. The third term is the average of cross terms involving products of deviations in $u$ and $v$ simultaneously. If we can make the assumptinn that the fluctuations in $u$ and $v$ are uncorrelated, then on the average we would expect to find as many approximately equal negative values for this tern as positive values, and we would expect the contribution to vanish in the limit of a large random selection of observations. If the fluctuations in the observations of $u, v, \ldots$ are uncorrelated, eq. (3.4) reduces to

$$
\sigma_{x}^{2}=\sigma_{u}^{2}\left(\frac{\partial x}{\partial u}\right)^{2}+\sigma_{v}^{2}\left(\frac{\partial x}{\partial v}\right)^{2}+\cdots
$$


Assuming target spin up and spin down polarizations are the same the analyzing power is given by

$$
A_{N}=\frac{\varepsilon}{P}
$$

where $\varepsilon$ is the left-right asymmetry and $P$ is the target polarization. However, the target polarizacion is not necessarily the same for spin up, $P \uparrow$, and spin down, Pt, cases. The difference between maximum positive and negative polarizations results from the asymetry of the ESR line and non linearity of the electronic system used to measure the polarization. The normalized yield for polarization up, $\mathrm{N} \uparrow$ is

$$
N+=N_{0}\left(I+A_{N} P+\right)+B .
$$

This "up" direction is more precisely uefined as the direction $\hat{n}$ (the unit normal to the scatcering plane), and $N_{o}$ is the unpolarized yield and $B$, the normalized background, is treated as being independent of the target polarization direction. Similarly, the normalized yield for polarization down, $\mathrm{N}+$ is

$$
N+=N_{0}\left(1-A_{N} P \downarrow\right)+B .
$$

Defining the average polarization $\bar{p}$, and polarization difference $A P$

$$
\begin{aligned}
& \bar{P}=\frac{P t+P t}{2}, \\
& \Delta P=P t-P t,
\end{aligned}
$$

we find

$$
\begin{aligned}
& \mathrm{Pt}=\overline{\mathrm{P}}+\frac{\Delta \mathrm{P}}{2} \\
& \mathrm{Pt}=\overline{\mathrm{P}}-\frac{\Delta \mathrm{P}}{2}
\end{aligned}
$$

and therefore the yields in terms of average polarization and polarization difference become

$$
\begin{aligned}
& N \uparrow=N_{0}\left[1+A_{N}\left(\bar{P}+\frac{\Delta P}{2}\right)\right]+B, \\
& N \downarrow=N_{0}\left[1-A_{N}\left(\bar{P}-\frac{\Delta P}{2}\right)\right]+B .
\end{aligned}
$$


Eliminating $N_{0}$ between these two equations and solving for $A_{N}$, we find

$$
A_{N}=\frac{N \uparrow-N \downarrow}{\bar{P}(N \uparrow+N \downarrow-2 B)-\frac{\Delta P}{2}(N \uparrow-N \downarrow)} .
$$

When $P \uparrow$ and $P \downarrow$ are the same, $\Delta P=0$ and this equation reduces to (3.6).

$A_{N}$ is a function of the independent variables $N \uparrow, N \downarrow, B, P \uparrow$, and $P+:$

$$
A_{N}=A_{N}(N \uparrow, N \downarrow, B, P \uparrow, P \uparrow)
$$

Thus, from eq. (3.5) it follows that

$$
\sigma_{A_{N}}^{2}=\left(\frac{\partial A_{N}}{\partial N \uparrow}\right)^{2} \sigma_{N \uparrow}^{2}+\left(\frac{\partial A_{N}}{\partial N \downarrow}\right)^{2} \underset{\sigma_{N} \downarrow}{2}+\left(\frac{\partial A_{N}}{\partial B}\right)^{2} \sigma_{B}^{2}+\left(\frac{\partial A_{N}}{\partial P \uparrow}\right)^{2} \sigma_{P \uparrow}^{2}+\left(\frac{\partial A_{N}}{\partial P \downarrow}\right)^{2} \sigma_{P \downarrow}^{2} .
$$

From (3.7) we find the derivatives

$$
\begin{aligned}
& \frac{\partial A_{N}}{\partial N \uparrow}=A_{N}^{2} \frac{2 \bar{P}(N \downarrow-B)}{(N \uparrow-N \downarrow)^{2}} \\
& \frac{\partial A_{N}}{\partial N \downarrow}=A_{N}^{2} \frac{-2 \bar{P}(N \uparrow-B)}{(N \uparrow-N \downarrow)^{2}} \\
& \frac{\partial A_{N}}{\partial B}=A_{N}^{2} \frac{2 \bar{P}}{\bar{N} \uparrow-N \downarrow} \\
& \frac{\partial A_{N}}{\partial P \uparrow}=A_{N}^{2} \frac{-(N \downarrow-B)}{N \uparrow-N \downarrow} \\
& \frac{\partial A_{N}}{\partial P \downarrow}=A_{N}^{2} \frac{-(N \uparrow-B)}{N \uparrow-N \downarrow}
\end{aligned}
$$

writing

$$
\sigma_{A_{N}}^{2}=\sigma_{A_{N}}^{-2}+\sigma_{A_{N}}^{\prime \prime 2}
$$

where

$$
\sigma_{A_{N}}^{2}=\left(\frac{\partial A}{\partial N \uparrow}\right)^{2} \sigma_{N \uparrow}^{2}+\left(\frac{\partial A}{\partial N \downarrow}\right)^{2} \sigma_{N \downarrow}^{2}+\left(\frac{\partial A}{\partial B}\right)^{2} \sigma_{B}^{2}
$$

and

$$
\sigma_{A_{N}}^{\prime \prime 2}=\left(\frac{\partial A_{N}}{\partial P \uparrow}\right)^{2} \sigma_{P \uparrow}^{2}+\left(\frac{\partial A_{N}}{\partial P \downarrow}\right)^{2} \sigma_{P \downarrow}^{2}
$$


one finds

$$
\sigma_{A_{N}}^{-}=\frac{2 \bar{P} A_{N}^{2}}{(N \uparrow-N \downarrow)^{2}}\left[(N \downarrow-B)^{2} \sigma_{N \uparrow}^{2}+(N \uparrow-B)^{2} \sigma_{N \downarrow}^{2}+(N \uparrow-N \downarrow)^{2} \sigma_{B}^{2}\right]^{1 / 2}
$$

and

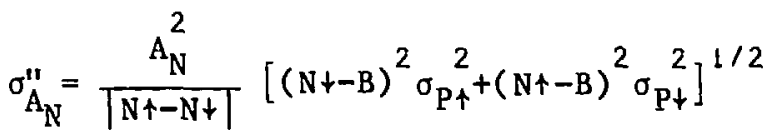

where $\mathrm{N} \uparrow$ is

$$
\mathrm{N}+=\frac{\text { Polarization Up Yield }}{\text { Corrected Monitor }} \text {. }
$$

The events occurred randomly in time and were described by a poisson distribution. Thus, for $\mathrm{N}$ events recorded in a detector, the absolute variance was $\sqrt{\mathrm{N}}$ and therefore

$$
\sigma_{\mathrm{N} \uparrow}=\frac{\sqrt{\text { Polarization Up Yield }}}{\text { Corrected Monitor }}
$$

and similar relations for $\mathrm{N} t, \sigma_{\mathrm{N}+}, \mathrm{B}$, and $\sigma_{\mathrm{B}}$. 


\subsection{Results and Conclusions}

The results of our measurements of the left-right asymmetry, $A_{N}$, of $\pi^{ \pm}$p elastic scattering at $471,547,625$, and $687 \mathrm{MeV} / \mathrm{c}$ incident pion momentum are given in tables $3.5-3.12$. The scattering angle was obtained from the LAS particle momentum taking energy loss in the target into account. The results are presented graphically in figures $3.14-3.21$, together with partial wave analysis predictions, these PWAs do not include our new experimental results in their data base. The errors presented in the tables involve only the statistical errors in the experiment. The systematic error due to the uncertainty in the absolute calibration of the target polazization 1 s $\pm 3 \%$. The polarization-asymetry theorem states that $A_{N}=P$, where $P$ is the recoil proton polarization measured with an unpolarized target. For this reason $A_{N}$ is sometimes called the polarization parameter.

The $\pi^{-} \mathrm{p}$ data are characterized by large values of the asymmetry parameter and rapid variation in angular dependence. At forward angles the angular dependence of the asymmetry parameter is similar at all incident momenta: in the range $0.2 \leqslant \cos \theta_{\mathrm{cm}} \leqslant 0.6$ the asymmetry parameter is negative and almost constant $(\sim-0.8)$, then it rises sharply, crossing zero near $\cos \theta_{\mathrm{cm}}=-0.2$ (at this angle the differential cross sections of $\pi^{-} p$ elastic scattering have a minimum). From 471 to $625 \mathrm{MeV} / \mathrm{c} \mathrm{A}_{\mathrm{N}}$ is large and positive at backward angles. There is a dramatic change at $687 \mathrm{MeV} / \mathrm{c}$ where it is seen that the 
asymmetry parameter varies very rapidly in the range of negative $\cos \theta_{\mathrm{cm}}$, crossing zero twice.

Our $\pi^{+} p$ results show that at backward angles the asymmetry parameter strongly depends on momentum. There is a dip in $A_{N}$ at backward angles in the regicn $\cos \theta_{\mathrm{cm}}=-0.4$ to -0.6 . When the momentum increases from 471 to $625 \mathrm{MeV} / \mathrm{c}$ this dip becomes deeper approaching the value -1 . For higher momenta this minimum decreases and then $A_{N}$ becomes positive. The origin of this rapid change of the asymmetry parameter is not understood yet.

As was mentioned earlier a complete set of experiments at a given momentum and angle are necessary to fully determine the scattering matrix. Generally one has a series of experiments at different momenta and angles to work with. To tie all of these data together, it is necessary to find some method of parameterizing the data. The partial wave analysis (PWA) is a fit of avallable experimental data using a limited number of partial wave amplitudes (or phase shifts).

It is interesting to compare the present data with the predictions of various recent phase shift analysis (PSA). The Karlsruhe-Helsinki ${ }^{5}$ phase shift analysis predictions are presented by the solid curves in figures $3.14-3.21$. To fill up the void caused by the lack of spin transfer data the $\mathrm{K}-\mathrm{H}$ PWA exploits Lorentz invariance, unitarity, crossing symmetry, analyticity and isospin invariance. The Carnegie-Mellon University and Lawrence Berkeley Laboratory, CMU-LBL, 
collaboration 6 PWA predictions are presented by the chain-dashed curves in the Figures. The CMU-LBL collaboration PWA uses analyticity at fixed $s$ in the variable $\cos \theta$. The Virginia Polytechnic Institute, 45 VPI, partial wave analysis results are presented in the figures by Jashed curves. The PSA's differ in the data base, the use of dispersion relations and in the incorporation of theoretical constraints from analyticity and isospin invariance.

Figures 3.14 - 3.19 show that the existing PWA reproduce the qualitative features of our experimental $\pi^{+} \mathrm{p}$ data. At $471 \mathrm{MeV} / \mathrm{c}$ the quantitative agreement is also good. For a numerical comparison of our data with the PWA predictions see table 3.5. At $547 \mathrm{MeV} / \mathrm{c}$ VPI solutions are in slightly better agreement with our results than the $\mathrm{K}-\mathrm{H}$ and CMU-LBL predictions. See table 3.6 for a numerical comparison. At $625 \mathrm{MeV} / \mathrm{c}$ again the VPI values are perhaps in better agreement with our data. Finally, at $687 \mathrm{MeV} / \mathrm{C} \mathrm{K}-\mathrm{H}$ predicts positive values for the asymmetry parameter at backward angles while our data indicate a negative value. The CMU-LBT, predictions are in better agreement with our measurements at this momentum.

For our $\pi^{-}$p results at $471 \mathrm{MeV} / \mathrm{c}$ comparison shows that all three phase shfit solutions equally well describe the experimental data around $90^{\circ}$. PWAs predictions indicate a minimum of -1 in the region $0.2 \leqslant \cos \theta_{\mathrm{cm}} \leqslant 0.6$ but our data shows a minimum not quite as low. At $547 \mathrm{MeV} / \mathrm{c}$ the agreement is good at backward angles, but the PWAs are lower by up to six standard deviations at forward angles. At $625 \mathrm{MeV} / \mathrm{c}$ 
at backward angles the disagreement is of the order of four standard deviations and at forward angles up to nine standard deviations. Finally at $687 \mathrm{MeV} / \mathrm{c}$ the agreement is gocis at forward angles, but PWAs are lower by up to four standard deviations at backward angles. From 471 to $625 \mathrm{MeV} / \mathrm{c}$ at backward angles $\mathrm{A}_{\mathrm{N}}$ is large and positive. There is a dramatic change between 625 and $687 \mathrm{MeV} / \mathrm{c}$. As the incident pion momentum increases, $A_{N}$ at backward angles becomes more negative approaching -1 .

There are daca from earlier works. Below our momentum region there exist the data of Amsler et $a .^{46}$ at 8 momenta from 188 to $427 \mathrm{MeV} / \mathrm{c}$, and Alder et al. 47 at 408 and $425 \mathrm{MeV} / \mathrm{c}$. At higher momenta Albrow et al. 48 at 23 momenta from 800 to $2400 \mathrm{MeV} / \mathrm{c}$, and also Martin et al. 49 otrer $\pi^{ \pm}$p polarization measurements within the momentum range of the present experiment have been made by the following: Martin et al. $49 \pi^{+} \mathrm{p}$ at 64 momenta Erom 600 to $2550 \mathrm{MeV} / \mathrm{c}$, Bekrenev et al.12 $\pi^{-} \mathrm{p}$ at 5 momenta from 573 to $726 \mathrm{MeV} / \mathrm{c}$, Abaev et al. $50 \mathrm{~m}^{+} \mathrm{p}$ at 7 momenta from 455 to $705 \mathrm{MeV} / \mathrm{c}$, We compare briefly the present results with the data from these experiments at the nearest available momenta. As we have seen the asymetry parameter varies very rapidly in our momentum region, therefore direct comparison of data is impossible if the beam momenta are not close enough. Figure 3.22 shows the results of the present experiment for $\pi^{+} \mathrm{p}$ at $625 \mathrm{MeV} / \mathrm{c}$ together with the results of Martin et al. 49 at $617 \mathrm{MeV} / \mathrm{c}$. It is not meaningful to compare numerically our data with the results of their work owing to their low statistical 
accuracy. Nevertheless one can see a different behavior of the angular dependence in the forward angles. The data of this work obviausly have a much higher precision.

Figure 3.23 shows the results of the present experiment for $\pi^{+} \mathrm{p}$ at $687 \mathrm{MeV} / \mathrm{c}$ together with the results of Abaev et al. 50 at $685 \mathrm{MeV} / \mathrm{c}$. Their measurements are restricted to backward angles. Our results agree within the errors. Such an agreement can be considered as evidence of the absence of large systematic errors. In figure 3.24 the results of the present experiment for It p at $687 \mathrm{MeV} / \mathrm{c}$ together with the results of Bekrenev ot $2: .^{12}$ at $685 \mathrm{MeV} / \mathrm{c}$ are presented. Their results have erroro greater than 0.1 . Our results are in good agreement with Bekrenev et al. data at forward angles, but their results are slightly lower than our measurements at backward angles. If their beam momentum in reality had been slightly higher than our beam momentum, this can easily account for the difference. In summary, the present data are in agreement with other resuits, but they have always substantlally higher precision. The data in this work were obtajned for a wide angular range and at precisely the same incident pion beam momentum as the previuusly measured elastic and charge exchange differential cross sections.

Charge independence in strong inceractions implies isospin reiations ancing the amplitudes of pion-nucleon scattering. Cutkosky 5 has pradi ted that isospin violations in $\pi \mathrm{N}$ scattering in the region 525-ico MeV due to $\pi^{0}-\eta^{0}$ mixing may be observable at backward angles. 
The deviations from isospin invariance need not be purely of electromagnetic origin. In fact it is currently believed that the masses of up and down quarks are different causing a small isospin violation. Our data together with the forthcoming data on $A_{N}\left(\pi^{-} p^{\circ} \pi^{0}\right)$ can be used to probe the triangle inequalities for a model independent test of the charge independence. 
Table $3 . j(a)$

Results of the measurement of the asymmetry parameter in $\pi^{+} \mathrm{p}$ elastic scattering at $471 \mathrm{MeV} / \mathrm{c}$ together with phase shift analysis predictions. Only statistical errors are 1isted. The systematical error due to the uncertainty in the absolute target polarization is $\pm 3 \%$.

\begin{tabular}{|c|c|c|c|c|c|c|c|c|}
\hline${ }^{\theta} \mathrm{cm}$ & $\Delta \theta_{\mathrm{cm}}$ & $\cos \theta_{\mathrm{CI}}$ & $\Delta \cos \theta_{\mathrm{cm}}$ & $\mathrm{A}_{\mathrm{N}}$ & $\sigma_{\mathrm{A}_{\mathrm{N}}}^{\prime}$ & $\mathrm{K}-\mathrm{H}$ & CMU-LBL & VPI \\
\hline 59.7 & $\begin{array}{l}+2.6 \\
-7.0\end{array}$ & 0.50 & $\begin{array}{l}+0.10 \\
-0.14\end{array}$ & 0.180 & 0.023 & 0.16 & 0.17 & 0.12 \\
\hline 65.3 & $\begin{array}{l}+5.7 \\
-3.0\end{array}$ & 0.42 & $\begin{array}{l}+0.05 \\
-0.09\end{array}$ & 0.161 & 0.023 & 0.18 & 0.20 & 0.14 \\
\hline 69.0 & $\begin{array}{l}+2.1 \\
-4.0\end{array}$ & 0.36 & $\begin{array}{l}+0.06 \\
-0.03\end{array}$ & 0.164 & 0.027 & 0.20 & 0.21 & 0.16 \\
\hline 73.4 & $\begin{array}{l}+3.5 \\
-2.4\end{array}$ & 0.29 & $\begin{array}{l}+0.04 \\
-0.06\end{array}$ & 0.196 & 0.028 & 0.23 & 0.24 & 0.18 \\
\hline 81.4 & $\begin{array}{l}+2.1 \\
-3.1\end{array}$ & 0.15 & $\begin{array}{l}+9.05 \\
-0.04\end{array}$ & 0.232 & 0.031 & 0.27 & 0.29 & 0.24 \\
\hline 85.7 & $\begin{array}{l}+3.4 \\
-2.2\end{array}$ & 0.07 & $\begin{array}{l}+0.04 \\
-0.06\end{array}$ & 0.267 & 0.036 & 0.29 & 0.32 & 0.28 \\
\hline 92.7 & $\begin{array}{l}+2.1 \\
-3.5\end{array}$ & -0.05 & $\begin{array}{l}+0.06 \\
-0.04\end{array}$ & 0.321 & 0.052 & 0.31 & 0.35 & 0.32 \\
\hline 97.0 & $\begin{array}{l}+3.3 \\
-2.3\end{array}$ & -0.12 & $\begin{array}{l}+0.04 \\
-0.06\end{array}$ & 0.217 & 0.053 & 0.30 & 0.35 & 0.33 \\
\hline 107.8 & $\begin{array}{l}+2.9 \\
-2.4\end{array}$ & -0.31 & $\begin{array}{l}+0.04 \\
-0.05\end{array}$ & 0.192 & 0.063 & 0.12 & 0.19 & 0.16 \\
\hline 121.5 & $\begin{array}{l}+3.0 \\
-2.3\end{array}$ & -0.52 & $\begin{array}{l}+0.03 \\
-0.04\end{array}$ & -0.184 & 0.042 & -0.17 & -0.11 & -0.14 \\
\hline 134.2 & $\begin{array}{l}+6.4 \\
-5.4\end{array}$ & -0.70 & $\begin{array}{l}+0.07 \\
-0.07\end{array}$ & -0.183 & 0.042 & -0.21 & -0.19 & -0.20 \\
\hline 154.0 & $\begin{array}{l}+9.5 \\
-6.0\end{array}$ & -0.90 & $\begin{array}{l}+0.05 \\
-0.06\end{array}$ & -0.062 & 0.040 & -0.13 & -0.15 & -0.13 \\
\hline
\end{tabular}


Tab1e 3.5(b)

Same as table $3.5(a)$ except that bins have not been divided.

\begin{tabular}{|c|c|c|c|c|c|c|c|c|}
\hline$\theta_{\mathrm{cm}}$ & $\Delta \theta_{\mathrm{cm}}$ & $\cos \theta_{\mathrm{cm}}$ & $\Delta \cos \theta_{\mathrm{cm}}$ & $\mathrm{A}_{\mathrm{N}}$ & $\sigma_{\mathrm{A}_{N}}^{-}$ & $\mathrm{K}-\mathrm{H}$ & CMU-LBL & VPI \\
\hline 62.6 & $\begin{array}{l}+8.5 \\
-9.8\end{array}$ & 0.46 & $\begin{array}{l}+0.14 \\
-0.14\end{array}$ & 0.173 & 0.017 & 0.17 & 0.13 & 0.13 \\
\hline 71.3 & $\begin{array}{l}+5.7 \\
-6.3\end{array}$ & 0.32 & $\begin{array}{l}+0.10 \\
-0.09\end{array}$ & 0.180 & 0.020 & 0.21 & 0.22 & 0.17 \\
\hline 83.5 & $\begin{array}{l}+5.7 \\
-5.1\end{array}$ & 0.11 & $\begin{array}{l}+0 .: 3 \\
-0.10\end{array}$ & 0.253 & 0.024 & 0.28 & 0.30 & 0.26 \\
\hline 94.7 & $\begin{array}{l}+5.6 \\
-5.5\end{array}$ & -0.08 & $\begin{array}{l}+0.10 \\
-0.10\end{array}$ & 0.273 & 0.038 & 0.31 & 0.35 & 0.33 \\
\hline 107.8 & $\begin{array}{l}+2.9 \\
-2.4\end{array}$ & -0.31 & $\begin{array}{l}+0.04 \\
-0.05\end{array}$ & 0.192 & 0.063 & 0.12 & 0.19 & 0.16 \\
\hline 121.5 & $\begin{array}{l}+3.0 \\
-2.3\end{array}$ & -0.52 & $\begin{array}{l}+0.03 \\
-0.04\end{array}$ & -0.184 & 0.042 & -0.17 & -0.11 & -0.14 \\
\hline 134.2 & $\begin{array}{l}+6.4 \\
-5.4\end{array}$ & -0.70 & $\begin{array}{l}+0.07 \\
-0.07\end{array}$ & -0.183 & 0.042 & -0.21 & -0.19 & -0.20 \\
\hline 154.0 & $\begin{array}{l}+9.5 \\
-6.0\end{array}$ & -0.90 & $\begin{array}{l}+0.05 \\
-0.06\end{array}$ & -0.062 & 0.040 & -0.13 & -0.15 & -0.13 \\
\hline
\end{tabular}


Table $3.6(\mathrm{a})$

Results of the measurement of the asymmetry parameter in $\pi^{+} \mathrm{p}$ elastic scattering at $547 \mathrm{MeV} / \mathrm{c}$ together with phase shift analysis predictions. Only statistical errors are listed. The systematical error due to the uncertainty in the absolute target polarization is $\pm 3 \%$.

\begin{tabular}{|c|c|c|c|c|c|c|c|c|}
\hline$\theta_{\mathrm{cm}}$ & $\Delta \theta_{\mathbf{c m}}$ & $\cos \theta_{\mathrm{cm}}$ & $\Delta \cos \theta_{\mathrm{cm}}$ & $A_{N}$ & $\sigma_{\mathrm{A}_{\mathrm{N}}}$ & $\mathrm{K}-\mathrm{H}$ & CMU-LBL & VPI \\
\hline 58.5 & $\begin{array}{l}+2.4 \\
-3.0\end{array}$ & 0.52 & $\begin{array}{l}+0.04 \\
-0.04\end{array}$ & 0.059 & 0.025 & 0.10 & 0.10 & 0.04 \\
\hline 62.9 & $\begin{array}{l}+3.2 \\
-2.0\end{array}$ & 0.46 & $\begin{array}{l}+0.03 \\
-0.05\end{array}$ & 0.101 & 0.034 & 0.11 & 0.10 & 0.04 \\
\hline $68.4^{\star}$ & $\begin{array}{l}+0.9 \\
-1.0\end{array}$ & 0.37 & $\begin{array}{l}+0.02 \\
-0.02\end{array}$ & 0.018 & 0.032 & 0.12 & 0.11 & 0.05 \\
\hline $70.3^{\star}$ & $\begin{array}{l}+0.9 \\
-1.0\end{array}$ & 0.34 & $\begin{array}{l}+0.02 \\
-0.02\end{array}$ & 0.042 & 0.027 & 0.13 & 0.11 & 0.05 \\
\hline 78.5 & $\begin{array}{l}+2.3 \\
-2.4\end{array}$ & 0.20 & $\begin{array}{l}+0.04 \\
-0.04\end{array}$ & 0.049 & 0.031 & 0.15 & $0 . \bar{i} 3$ & 0.07 \\
\hline 82.6 & $\begin{array}{l}+2.7 \\
-1.9\end{array}$ & 0.13 & $\begin{array}{l}+0.03 \\
-0.05\end{array}$ & 0.041 & 0.046 & 0.16 & 0.14 & 0.08 \\
\hline $88.3^{*}$ & $\begin{array}{l}+1.4 \\
-2.1\end{array}$ & 0.03 & $\begin{array}{l}+0.04 \\
-0.02\end{array}$ & 0.087 & 0.041 & 0.18 & 0.17 & 0.11 \\
\hline $91.3^{\star}$ & $\begin{array}{l}+2.0 \\
-1.5\end{array}$ & -0.02 & $\begin{array}{l}+0.03 \\
-0.04\end{array}$ & 0.139 & 0.036 & 0.17 & 0.16 & 0.10 \\
\hline $108.8^{\star}$ & $\begin{array}{l}+2.0 \\
-1.5\end{array}$ & -0.32 & $\begin{array}{l}+0.02 \\
-0.03\end{array}$ & -0.109 & 0.053 & -0.01 & -0.09 & -0.15 \\
\hline 118.3 & $\begin{array}{l}+1.9 \\
-2.7\end{array}$ & -0.47 & $\begin{array}{l}+0.04 \\
-0.03\end{array}$ & -0.298 & 0.055 & -0.40 & -0.42 & -0.44 \\
\hline 122.2 & $\begin{array}{l}+2.3 \\
-2.0\end{array}$ & -0.53 & $\begin{array}{l}+0.03 \\
-0.03\end{array}$ & -0.403 & 0.058 & -0.48 & -0.46 & -0.48 \\
\hline 136.2 & $\begin{array}{l}+5.5 \\
-5.3\end{array}$ & -0.72 & $\begin{array}{l}+0.07 \\
-0.06\end{array}$ & -0.268 & 0.034 & -0.44 & -0.39 & -0.40 \\
\hline
\end{tabular}

*Protons were detected in the LAS. 
Table $3.6(b)$

Same as table 3.6(a) except that bins have not been divided.

\begin{tabular}{|c|c|c|c|c|c|c|c|c|}
\hline$\theta_{\mathrm{cm}}$ & $\Delta \theta_{\mathrm{cm}}$ & $\cos \theta_{\mathrm{cm}}$ & $\Delta \cos \theta_{\mathrm{cm}}$ & $\mathrm{A}_{\mathrm{N}}$ & $\sigma_{\mathrm{A}_{\mathrm{N}}}^{-}$ & $\mathrm{K}-\mathrm{H}$ & CMU-LBL & VPI \\
\hline 60.1 & $\begin{array}{l}+5.9 \\
-4.6\end{array}$ & 0.50 & $\begin{array}{l}+0.07 \\
-0.09\end{array}$ & 0.072 & 0.021 & 0.10 & 0.10 & 0.04 \\
\hline $69.5^{*}$ & $\begin{array}{l}+1.7 \\
-2.1\end{array}$ & 0.35 & $\begin{array}{l}+0.04 \\
-0.03\end{array}$ & 0.035 & 0.021 & 0.13 & 0.11 & 0.05 \\
\hline 80.0 & $\begin{array}{l}+5.3 \\
-3.9\end{array}$ & 0.17 & $\begin{array}{l}+0.07 \\
-0.09\end{array}$ & 0.057 & 0.026 & 0.15 & 0.14 & 0.07 \\
\hline $90.1^{*}$ & $\begin{array}{l}+3.2 \\
-3.8\end{array}$ & 0.00 & $\begin{array}{l}+0.07 \\
-0.06\end{array}$ & 0.112 & 0.028 & 0.17 & 0.17 & 0.10 \\
\hline $108.8^{*}$ & $\begin{array}{l}+2.0 \\
-1.5\end{array}$ & -0.32 & $\begin{array}{l}+0.02 \\
-0.03\end{array}$ & -0.109 & 0.053 & -0.01 & -0.09 & -0.15 \\
\hline 120.4 & $\begin{array}{l}+4.1 \\
-4.8\end{array}$ & -0.51 & $\begin{array}{l}+0.07 \\
-0.06\end{array}$ & -0.347 & 0.041 & -0.46 & -0.46 & -0.47 \\
\hline 136.2 & $\begin{array}{l}+5.5 \\
-5.3\end{array}$ & -0.72 & $\begin{array}{l}+0.07 \\
-0.06\end{array}$ & -0.268 & 0.034 & -0.44 & -0.39 & -0.40 \\
\hline
\end{tabular}

* Protons were detected in the LAS. 


\section{Table $3.7(a)$}

Results of the measurement of the asymmetry parameter in $\pi^{+} p$ elastic scattering at $625 \mathrm{MeV} / \mathrm{c}$ together with phase shift analysis predictions. Only statistical errors are lisced. The systematical error due to the uncertainty in the absolute target polarization is $\pm 3 \%$.

\begin{tabular}{|c|c|c|c|c|c|c|c|c|}
\hline$\theta_{\mathrm{cm}}$ & $\Delta \theta_{\mathrm{cm}}$ & $\cos \theta_{\mathrm{cm}}$ & $\Delta \cos \theta_{\mathrm{cm}}$ & $A_{N}$ & $\sigma_{A_{N}}$ & $\mathrm{~K}-\mathrm{H}$ & CMU-LBL & VPI \\
\hline 48.2 & $\begin{array}{l}+2.3 \\
-3.3\end{array}$ & 0.67 & $\begin{array}{l}+0.04 \\
-0.03\end{array}$ & -0.004 & 0.043 & -0.02 & 0.04 & 0.00 \\
\hline 52.7 & $\begin{array}{l}+3.4 \\
-2.2\end{array}$ & 0.61 & $\begin{array}{l}+0.03 \\
-0.05\end{array}$ & -0.005 & 0.070 & -0.02 & 0.03 & -0.02 \\
\hline $65.3^{*}$ & $\begin{array}{l}+0.8 \\
-1.0\end{array}$ & 0.42 & $\begin{array}{l}+0.02 \\
-0.01\end{array}$ & -0.026 & 0.035 & -0.03 & 0.01 & -0.05 \\
\hline $66.6^{*}$ & $\begin{array}{l}+1.3 \\
-0.6\end{array}$ & 0.40 & $\begin{array}{l}+0.01 \\
-0.02\end{array}$ & -0.085 & 0.043 & -0.03 & 0.01 & -0.05 \\
\hline 77.0 & $\begin{array}{l}+1.9 \\
-2.7\end{array}$ & 0.22 & $\begin{array}{l}+0.05 \\
-0.03\end{array}$ & -0.040 & 0.048 & -0.03 & 0.00 & -0.07 \\
\hline 80.9 & $\begin{array}{l}+2.8 \\
-2.0\end{array}$ & 0.16 & $\begin{array}{l}+0.03 \\
-0.05\end{array}$ & -0.099 & 0.054 & -0.04 & 0.00 & -0.08 \\
\hline 103.5 & $\begin{array}{l}+1.9 \\
-2.2\end{array}$ & -0.23 & $\begin{array}{l}+0.04 \\
-0.03\end{array}$ & -0.475 & 0.119 & -0.08 & -0.14 & -0.27 \\
\hline 107.3 & $\begin{array}{l}+2.0 \\
-1.9\end{array}$ & -0.30 & $\begin{array}{l}+0.03 \\
-0.03\end{array}$ & -0.545 & 0.141 & -0.16 & -0.33 & -0.47 \\
\hline 120.5 & $\begin{array}{l}+1.7 \\
-1.8\end{array}$ & -0.51 & $\begin{array}{l}+0.03 \\
-0.03\end{array}$ & -0.737 & 0.159 & -0.85 & -0.93 & -0.85 \\
\hline i23.9 & $\begin{array}{l}+2.0 \\
-1.7\end{array}$ & -0.56 & $\begin{array}{l}+0.03 \\
-0.03\end{array}$ & -0.699 & 0.151 & -0.77 & -0.79 & -0.71 \\
\hline 150.1 & $\begin{array}{l}+3.6 \\
-2.9\end{array}$ & -0.87 & $\begin{array}{l}+0.03 \\
-0.03\end{array}$ & -0.289 & 0.158 & -0.45 & -0.38 & -0.41 \\
\hline
\end{tabular}

* Protons were detected in the LAS. 
Table 3.7(b)

Same as table 3.7(a) except that bins have not been divided.

\begin{tabular}{|c|c|c|c|c|c|c|c|c|}
\hline$\theta_{\mathrm{crn}}$ & $\Delta \theta_{\mathrm{cm}}$ & $\cos \theta \mathrm{cm}$ & $\Delta \cos \theta_{\mathrm{cm}}$ & $\mathrm{A}_{\mathrm{N}}$ & $\sigma_{\mathrm{A}_{N}}$ & $\mathrm{~K}-\mathrm{H}$ & CMU-LBI & VPI \\
\hline 50.1 & $\begin{array}{l}+6.0 \\
-5.1\end{array}$ & 0.64 & $\begin{array}{l}+0.07 \\
-0.08\end{array}$ & -0.014 & 0.038 & -0.02 & 0.04 & -0.01 \\
\hline $65.8^{*}$ & $\begin{array}{l}+2.1 \\
-1.5\end{array}$ & $0.4 !$ & $\begin{array}{l}+0.02 \\
-0.03\end{array}$ & -0.063 & 0.028 & -0.03 & 0.01 & -0.05 \\
\hline 78.9 & $\begin{array}{l}+4.7 \\
-4.6\end{array}$ & 0.19 & $\begin{array}{l}+0.08 \\
-0.08\end{array}$ & -0.039 & 0.036 & -0.03 & 0.00 & -0.08 \\
\hline 105.0 & $\begin{array}{l}+4.3 \\
-3.8\end{array}$ & -0.26 & $\begin{array}{l}+0.06 \\
-0.07\end{array}$ & -0.512 & 0.097 & -0.11 & -0.21 & -0.32 \\
\hline 122.1 & $\begin{array}{l}+3.7 \\
-3.5\end{array}$ & -0.53 & $\begin{array}{l}+0.05 \\
-0.05\end{array}$ & -0.720 & 0.115 & -0.81 & -0.86 & -0.78 \\
\hline 150.1 & $\begin{array}{l}+3.6 \\
-2.9\end{array}$ & -0.87 & $\begin{array}{l}+0.03 \\
-0.03\end{array}$ & -0.289 & 0.158 & -0.45 & -0.38 & -0.41 \\
\hline
\end{tabular}

*Protons were detected in the LAS. 
Table 3.8(a)

Results of the measurement of the asymmetry parameter in $\pi^{+} \mathrm{p}$ elastic scattering at $687 \mathrm{MeV} / \mathrm{c}$ together with phase shift analysis predictions. only statistical errors are listed. The systematical error due to the uncertainty in the absolute target polarization is $\pm 3 \%$.

\begin{tabular}{|c|c|c|c|c|c|c|c|c|}
\hline$\theta_{\mathrm{cm}}$ & $\Delta \theta_{\mathrm{cm}}$ & $\cos \theta_{\mathrm{cm}}$ & $\Delta \cos \theta_{\mathrm{cm}}$ & $\mathrm{A}_{\mathrm{N}}$ & $\sigma_{\mathrm{A}_{\mathrm{N}}}^{-}$ & $\mathrm{K}-\mathrm{H}$ & CMU-LBL & VPI \\
\hline $57.6^{*}$ & $\begin{array}{l}+1.5 \\
-1.6\end{array}$ & 0.54 & $\begin{array}{l}+0.02 \\
-0.02\end{array}$ & -0.097 & 0.060 & -0.10 & -0.04 & -0.09 \\
\hline $60.7^{*}$ & $\begin{array}{l}+1.6 \\
-1.7\end{array}$ & 0.49 & $\begin{array}{l}+0.03 \\
-0.03\end{array}$ & -0.110 & 0.064 & -0.11 & -0.05 & -0.11 \\
\hline $75.7^{*}$ & $\begin{array}{l}+0.9 \\
-1.1\end{array}$ & 0.25 & $\begin{array}{l}+0.02 \\
-0.02\end{array}$ & -0.124 & 0.065 & -0.16 & -0.08 & -0.16 \\
\hline $77.7^{*}$ & $\begin{array}{l}+1.0 \\
-1.1\end{array}$ & 0.21 & $\begin{array}{l}+0.02 \\
-0.02\end{array}$ & -0.166 & 0.081 & -0.17 & -0.08 & -0.16 \\
\hline $79.7^{*}$ & $\begin{array}{l}+1.1 \\
-1.0\end{array}$ & 0.18 & $\begin{array}{l}+0.02 \\
-0.02\end{array}$ & -0.241 & 0.101 & -0.17 & -0.09 & -0.17 \\
\hline 97.7 & $\begin{array}{l}+1.9 \\
-2.3\end{array}$ & -0.13 & $\begin{array}{l}+0.04 \\
-0.03\end{array}$ & -0.193 & 0.047 & -0.09 & -0.07 & -0.17 \\
\hline 101.3 & $\begin{array}{l}+2.5 \\
-1.7\end{array}$ & -0.19 & $\begin{array}{l}+0.03 \\
-0.04\end{array}$ & -0.094 & 0.057 & 0.00 & -0.06 & -0.13 \\
\hline 112.4 & $\begin{array}{l}+4.7 \\
-4.7\end{array}$ & -0.38 & $\begin{array}{l}+0.08 \\
-0.07\end{array}$ & 0.132 & 0.147 & 0.62 & 0.03 & 0.35 \\
\hline 133.6 & $\begin{array}{l}+1.7 \\
-2.0\end{array}$ & -0.69 & $\begin{array}{l}+0.03 \\
-0.02\end{array}$ & -0.141 & 0.100 & 0.17 & $-0.1 ?$ & -0.01 \\
\hline 137.0 & $\begin{array}{l}+2.1 \\
-1.8\end{array}$ & -0.73 & $\begin{array}{l}+0.02 \\
-0.02\end{array}$ & -0.172 & 0.108 & 0.12 & -0.14 & -0.07 \\
\hline 156.8 & $\begin{array}{l}+4.1 \\
-3.1\end{array}$ & -0.92 & $\begin{array}{l}+0.02 \\
-0.03\end{array}$ & -0.288 & 0.059 & -0.04 & -0.34 & -0.36 \\
\hline
\end{tabular}

*Protons were detected in the LAS. 


\section{Table $3.8(\mathrm{~b})$}

Same as table 3.8 (a) except that birs have not been divided.

\begin{tabular}{|c|c|c|c|c|c|c|c|c|}
\hline${ }^{\theta} \mathrm{cm}$ & $\Delta \theta_{\mathrm{cm}}$ & $\cos \theta_{\mathrm{cm}}$ & $\Delta \cos \theta_{\mathrm{cm}}$ & $A_{N}$ & $\sigma^{-A_{N}}$ & $\mathrm{~K}-\mathrm{H}$ & CMU-LBL & VPI \\
\hline $59.3^{*}$ & $\begin{array}{l}+3.1 \\
-3.3\end{array}$ & 0.51 & $\begin{array}{l}+0.05 \\
-0.05\end{array}$ & -0.112 & 0.045 & -0.10 & -0.04 & -0.10 \\
\hline $77.8^{*}$ & $\begin{array}{l}+3.0 \\
-3.1\end{array}$ & 0.21 & $\begin{array}{l}+0.05 \\
-0.05\end{array}$ & -0.168 & 0.046 & -0.17 & -0.08 & -0.16 \\
\hline 99.1 & $\begin{array}{l}+4.6 \\
-3.7\end{array}$ & -0.16 & $\begin{array}{l}+0.07 \\
-0.08\end{array}$ & -0.141 & 0.037 & -0.07 & -0.07 & -0.16 \\
\hline 112.4 & $\begin{array}{l}+4.7 \\
-4.7\end{array}$ & -0.38 & $\begin{array}{l}+0.08 \\
-0.07\end{array}$ & 0.132 & 0.147 & $0 . \dot{u} 2$ & 0.03 & 0.35 \\
\hline 135.4 & $\begin{array}{l}+3.7 \\
-3.8\end{array}$ & -0.71 & $\begin{array}{l}+0.05 \\
-0.04\end{array}$ & -0.146 & 0.080 & 0.14 & -0.13 & -0.05 \\
\hline 156.8 & $\begin{array}{l}+4.1 \\
-3.1\end{array}$ & -0.92 & $\begin{array}{l}+0.02 \\
-0.03\end{array}$ & -0.288 & 0.059 & -0.04 & -0.34 & -0.36 \\
\hline
\end{tabular}

* Protons were detected in the LAS. 
Results of the measurement of the asymmetry parameter in $\pi^{-} p$ elastic scattering at $47 \mathrm{l} \mathrm{MeV} / \mathrm{c}$ together with phase shift analysis predictions. Only statistical errors are 1isted. The systematical error due to the uncertainty in the absolute target polarization is $\pm 3 \%$.

\begin{tabular}{|c|c|c|c|c|c|c|c|c|}
\hline$\theta_{\mathrm{CII}}$ & $\Delta \theta_{\mathrm{cm}}$ & $\cos \theta_{\mathrm{cm}}$ & $\Delta \cos \theta_{\mathrm{cm}}$ & $\mathrm{A}_{\mathrm{N}}$ & $\sigma_{A_{N}}^{\prime}$ & $\mathrm{K}-\mathrm{H}$ & CMU-LBL & VPI \\
\hline 59.9 & $\begin{array}{l}+1.8 \\
-3.1\end{array}$ & 0.50 & $\begin{array}{l}+0.05 \\
-0.03\end{array}$ & -0.820 & 0.022 & -0.96 & -0.90 & -0.92 \\
\hline 63.9 & $\begin{array}{l}+2.6 \\
-2.2\end{array}$ & 0.44 & $\begin{array}{l}+0.03 \\
-0.04\end{array}$ & -0.806 & 0.020 & -0.93 & -0.87 & -0.89 \\
\hline 69.5 & $\begin{array}{l}+2.0 \\
-3.0\end{array}$ & 0.35 & $\begin{array}{l}+0.05 \\
-0.03\end{array}$ & -0.802 & 0.017 & -0.87 & -0.81 & -0.83 \\
\hline 73.6 & $\begin{array}{l}+2.8 \\
-2.1\end{array}$ & 0.28 & $\begin{array}{l}+0.03 \\
-0.05\end{array}$ & -0.718 & 0.019 & -0.81 & -0.76 & -0.78 \\
\hline 82.2 & $\begin{array}{l}+1.8 \\
-2.4\end{array}$ & 0.14 & $\begin{array}{l}+0.04 \\
-0.03\end{array}$ & -0.566 & 0.021 & -0.65 & -0.60 & -0.63 \\
\hline 86.0 & $\begin{array}{l}+2.7 \\
-2.0\end{array}$ & 0.07 & $\begin{array}{l}+0.03 \\
-0.05\end{array}$ & -0.474 & 0.024 & -0.55 & -0.50 & -0.53 \\
\hline 91.1 & $\begin{array}{l}+1.7 \\
-2.4\end{array}$ & -0.02 & $\begin{array}{l}+0.00 \\
-0.03\end{array}$ & -0.332 & 0.026 & -0.39 & -0.34 & -0.37 \\
\hline 94.7 & $\begin{array}{l}+2.4 \\
-1.8\end{array}$ & -0.08 & $\begin{array}{l}+0.03 \\
-0.04\end{array}$ & -0.191 & 0.027 & -0.26 & -0.21 & -0.25 \\
\hline 119.3 & $\begin{array}{l}+2.0 \\
-2.6\end{array}$ & -0.49 & $\begin{array}{l}+0.04 \\
-0.03\end{array}$ & 0.661 & 0.072 & 0.68 & 0.69 & 0.70 \\
\hline 123.2 & $\begin{array}{l}+2.3 \\
-2.0 \\
+4.1\end{array}$ & -0.55 & $\begin{array}{l}+0.03 \\
-0.03\end{array}$ & 0,672 & 0.085 & 0.70 & 0.70 & 0.71 \\
\hline 134.5 & $\begin{array}{l}+4.1 \\
-3.9\end{array}$ & -0.70 & $\begin{array}{l}+0.05 \\
-0.05\end{array}$ & 0.588 & 0.055 & 0.62 & 0.58 & 0.59 \\
\hline 154.1 & $\begin{array}{l}+4.2 \\
-3.6\end{array}$ & -0.90 & $\begin{array}{l}+0.03 \\
-0.03\end{array}$ & 0.487 & 0.036 & 0.33 & 0.31 & 0.28 \\
\hline
\end{tabular}


Table $3.9(b)$

Same as table 3.9(a) except that bins have not been divided.

\begin{tabular}{|c|c|c|c|c|c|c|c|c|}
\hline$\theta_{\mathrm{cm}}$ & $\Delta \theta_{\mathrm{cm}}$ & $\cos \theta_{\mathrm{cm}}$ & $\Delta \cos \theta_{\mathrm{cm}}$ & $\mathrm{A}_{\mathrm{N}}$ & $\sigma_{\mathrm{A}_{\mathrm{N}}}$ & $\mathrm{K}-\mathrm{H}$ & CMU-LBL & VPI \\
\hline 62.2 & $\begin{array}{l}+4.3 \\
-5.4\end{array}$ & 0.47 & $\begin{array}{l}+0.08 \\
-0.07\end{array}$ & -0.809 & 0.016 & -0.94 & -0.88 & -0.90 \\
\hline 71.6 & $\begin{array}{l}+4.9 \\
-5.1\end{array}$ & 0.32 & $\begin{array}{l}+0.08 \\
-0.08\end{array}$ & -0.763 & 0.013 & -0.84 & -0.79 & -0.81 \\
\hline 84.0 & $\begin{array}{l}+4.7 \\
-4.2\end{array}$ & 0.10 & $\begin{array}{l}+0.07 \\
-0.08\end{array}$ & -0.525 & 0.017 & -0.61 & -0.56 & -0.58 \\
\hline 92.9 & $\begin{array}{l}+4.1 \\
-4.2\end{array}$ & -0.05 & $\begin{array}{l}+0.07 \\
-0.07\end{array}$ & -0.262 & 0.020 & -0.33 & -0.28 & -0.31 \\
\hline 121.1 & $\begin{array}{l}+4.4 \\
-4.4\end{array}$ & -0.52 & $\begin{array}{l}+0.07 \\
-0.06\end{array}$ & 0.657 & 0.056 & 0.69 & 0.70 & 0.71 \\
\hline 134.5 & $\begin{array}{l}+4.1 \\
-3.9\end{array}$ & -0.70 & $\begin{array}{l}+0.05 \\
-0.05\end{array}$ & 0.588 & 0.055 & 0.62 & 0.58 & 0.59 \\
\hline 154.1 & $\begin{array}{l}+4.2 \\
-3.6\end{array}$ & -0.90 & $\begin{array}{l}+0.03 \\
-0.03\end{array}$ & 0.487 & 0.036 & 0.33 & 0.31 & 0.28 \\
\hline
\end{tabular}




\section{Table $3.10(a)$}

Results of the measurement of the asymmetry parameter in $\pi^{-} p$ elastic scattering at $5: ; \mathrm{MeV} / \mathrm{e}$ together with phase shift analysis predictions. only statistical errors are listed. The systenatical error due to the uncertainty in the absolute rarget polarization is $\pm 3 \%$.

\begin{tabular}{|c|c|c|c|c|c|c|c|c|}
\hline$\theta_{\mathrm{cm}}$ & $\Delta \theta_{\mathrm{cm}}$ & $\cos \theta_{\mathrm{cm}}$ & $\Delta \cos \theta_{\mathrm{cm}}$ & ${ }^{A} N$ & $\sigma_{A_{N}}^{-}$ & $\mathrm{K}-\mathrm{H}$ & CMU-LBL & VPI \\
\hline 53.5 & $\begin{array}{l}+1.5 \\
-1.9\end{array}$ & 0.59 & $\begin{array}{l}+0.02 \\
-0.03\end{array}$ & -0.752 & 0.036 & -0.90 & -0.99 & -0.98 \\
\hline 56.7 & $\begin{array}{l}+2.0 \\
-1.7\end{array}$ & 0.55 & $\begin{array}{l}+0.02 \\
-0.03\end{array}$ & -0.732 & 0.034 & -0.91 & -0.99 & -0.98 \\
\hline 62.5 & $\begin{array}{l}+4.3 \\
-3.8\end{array}$ & 0.46 & $\begin{array}{l}+0.06 \\
-0.07\end{array}$ & -0.846 & 0.021 & -0.92 & -0.97 & -0.98 \\
\hline 71.9 & $\begin{array}{l}+1.8 \\
-2.6\end{array}$ & 0.31 & $\begin{array}{l}+0.04 \\
-0.03\end{array}$ & -0.784 & 0.027 & -0.89 & -0.91 & -0.93 \\
\hline 75.9 & $\begin{array}{l}+2.5 \\
-2.2\end{array}$ & 0.24 & $\begin{array}{l}+0.04 \\
-0.05\end{array}$ & -0.802 & 0.027 & -0.86 & -0.87 & -0.90 \\
\hline 82.6 & $\begin{array}{l}+1.2 \\
-1.5\end{array}$ & 0.13 & $\begin{array}{l}+0.02 \\
-0.02\end{array}$ & -0.635 & 0.025 & -0.77 & -0.76 & -0.80 \\
\hline 85.2 & $\begin{array}{l}+1.6 \\
-1.4\end{array}$ & 0.08 & $\begin{array}{l}+0.03 \\
-0.03\end{array}$ & -0.612 & 0.021 & -0.71 & -0.69 & -0.75 \\
\hline 88.1 & $\begin{array}{l}+1.8 \\
-1.3\end{array}$ & 0.03 & $\begin{array}{l}+0.02 \\
-0.03\end{array}$ & -0.547 & 0.032 & -0.64 & -0.62 & -0.66 \\
\hline 93.5 & $\begin{array}{l}+1.6 \\
-1.8\end{array}$ & -0.06 & $\begin{array}{l}+0.03 \\
-0.03\end{array}$ & -0.403 & 0.058 & -0.43 & -0.40 & -0.46 \\
\hline 96.5 & $\begin{array}{l}+1.6 \\
-1.4\end{array}$ & -0.11 & $\begin{array}{l}+0.02 \\
-0.03\end{array}$ & -0.402 & 0.094 & -0.27 & -0.25 & $-0.3 i)$ \\
\hline 105.0 & $\begin{array}{l}+2.3 \\
-2.7\end{array}$ & -7.26 & $\begin{array}{l}+0.05 \\
-0.04\end{array}$ & 0.268 & 0.096 & 0.32 & 0.32 & 0.28 \\
\hline $118.2^{\star}$ & $\begin{array}{l}+2.0 \\
-2.2\end{array}$ & -0.47 & $\begin{array}{l}+0.03 \\
-0.03\end{array}$ & 0.746 & 0.092 & 0.97 & 0.98 & 0.97 \\
\hline $122.3^{*}$ & $\begin{array}{l}+2.2 \\
-2.1\end{array}$ & -0.53 & $\begin{array}{l}+0.03 \\
-0.04\end{array}$ & 0.955 & 0.094 & 0.97 & 0.97 & 0.97 \\
\hline 136.9 & $\begin{array}{l}+2.0 \\
-1.7\end{array}$ & -0.79 & $\begin{array}{l}+0.02 \\
-0.02\end{array}$ & 0.572 & 0.042 & 0.63 & 0.61 & 0.64 \\
\hline 140.6 & $\begin{array}{l}+2.3 \\
-1.7\end{array}$ & -0.77 & $\begin{array}{l}+0.02 \\
-0.03\end{array}$ & 0.441 & 0.054 & 0.54 & 0.53 & 0.54 \\
\hline 153.3 & $\begin{array}{l}+4.4 \\
-\therefore .8\end{array}$ & -0.89 & $\begin{array}{l}+0.02 \\
-0.03\end{array}$ & 0.183 & 0.028 & 0.30 & 0.30 & 0.29 \\
\hline
\end{tabular}

* Single arm measurement. 
Table $3.10(b)$

Same as table 3.10(a) except that bins have not been divided.

\begin{tabular}{|c|c|c|c|c|c|c|c|c|}
\hline$\theta_{\text {сл }}$ & $\Delta \theta_{\mathrm{cm}}$ & $\cos ^{\theta} \mathrm{cm}$ & $\Delta \cos \theta_{\mathrm{cm}}$ & $A_{N}$ & ${ }^{\alpha} \dot{A}_{N}$ & $\mathrm{~K}-\mathrm{H}$ & CMU-LBL & VPI \\
\hline $55 . ?$ & $\begin{array}{l}+3.5 \\
-3.6\end{array}$ & 0.57 & $\begin{array}{l}+0.05 \\
-0.05\end{array}$ & -0.747 & 0.026 & -0.91 & -0.99 & -0.98 \\
\hline 62.5 & $\begin{array}{l}+4.3 \\
-3.8\end{array}$ & 0.46 & $\begin{array}{l}+0.06 \\
-0.07\end{array}$ & -0.846 & 0.021 & -0.92 & -0.97 & -0.98 \\
\hline 74.1 & $\begin{array}{l}+4.3 \\
-4.8\end{array}$ & 0.27 & $\begin{array}{l}+0.07 \\
-0.07\end{array}$ & -0.786 & 0.019 & -0.87 & -0.89 & -0.92 \\
\hline 85.1 & $\begin{array}{l}+4.8 \\
-4.0\end{array}$ & 0.09 & $\begin{array}{l}+0.06 \\
-0.79\end{array}$ & -0.598 & 0.015 & -0.71 & -0.70 & -0.75 \\
\hline 94.9 & $\begin{array}{l}+3.2 \\
-3.2\end{array}$ & -0.09 & $\begin{array}{l}+0.06 \\
-0.06\end{array}$ & -0.424 & 0.057 & -0.36 & -0.34 & -0.40 \\
\hline 105.0 & $\begin{array}{l}+2.3 \\
-2.7\end{array}$ & -0.26 & $\begin{array}{l}+0.05 \\
-0.04\end{array}$ & 0.268 & 0.096 & 0.32 & 0.32 & 0.28 \\
\hline $120.2^{\star}$ & $\begin{array}{l}+4.3 \\
-4.2\end{array}$ & -0.50 & $\begin{array}{l}+0.06 \\
-0.07\end{array}$ & 0.857 & 0.069 & 0.99 & 1.00 & l. .00 \\
\hline 138.2 & $\begin{array}{l}+4.7 \\
-2.9\end{array}$ & -0.75 & $\begin{array}{l}+0.04 \\
-0.05\end{array}$ & 0.530 & 0.033 & 0.60 & 0.58 & 0.60 \\
\hline 153.3 & $\begin{array}{l}+4.4 \\
-2.8\end{array}$ & -0.89 & $\begin{array}{l}+0.02 \\
-0.03\end{array}$ & 0.183 & 0.028 & 0.30 & 0.30 & 0.29 \\
\hline
\end{tabular}

${ }^{\star}$ Single arm measurement. 
Table $3.11(\mathrm{a})$

Results of the measurement of the asymmetry parameter in $\pi^{-} p$ elastic scattering at $625 \mathrm{MeV} / \mathrm{c}$ together with phase shift analysis predictions. only statistical errors are listed. The systematical error due to the uncertainty in the absolute target polarization is $\pm 3 \%$.

\begin{tabular}{|c|c|c|c|c|c|c|c|c|}
\hline$\theta_{\mathrm{crm}}$ & $\Delta \theta_{\mathrm{CIn}}$ & $\cos \theta_{\mathrm{cm}}$ & $\Delta \cos \theta_{\mathrm{cm}}$ & $\mathrm{A}_{\mathrm{N}}$ & $\sigma_{\mathrm{A}_{\mathrm{N}}}^{-}$ & $\mathrm{K}-\mathrm{H}$ & CMU-LBL & VPI \\
\hline 51.8 & $\begin{array}{l}+2.8 \\
-5.5\end{array}$ & $0 . € 2$ & $\begin{array}{l}+0.07 \\
-0.04\end{array}$ & -0.813 & 0.033 & -0.77 & -0.86 & -0.88 \\
\hline 57.3 & $\begin{array}{l}+4.4 \\
-2.7\end{array}$ & 0.54 & $\begin{array}{l}+0.04 \\
-0.07\end{array}$ & -0.909 & 0.043 & -0.81 & -0.89 & -0.91 \\
\hline 65.9 & $\begin{array}{l}+2.4 \\
-4.2\end{array}$ & 0.41 & $\begin{array}{l}+0.06 \\
-0.04\end{array}$ & -0.798 & 0.014 & -0.84 & -0.91 & -0.93 \\
\hline 70.7 & $\begin{array}{l}+3.6 \\
-2.4\end{array}$ & 0.33 & $\begin{array}{l}+0.04 \\
-0.06\end{array}$ & -0.776 & 0.016 & -0.85 & -0.90 & -0.93 \\
\hline 79.3 & $\begin{array}{l}+2.2 \\
-4.0\end{array}$ & 0.18 & $\begin{array}{l}+0.07 \\
-0.03\end{array}$ & -0.700 & 0.042 & -0.83 & -0.85 & -0.88 \\
\hline 83.9 & $\begin{array}{l}+3.2 \\
-2.4\end{array}$ & 0.11 & $\begin{array}{l}+0.04 \\
-0.06\end{array}$ & -0.663 & 0.028 & -0.79 & -0.79 & -0.83 \\
\hline 117.5 & $\begin{array}{l}+1.8 \\
-1.8\end{array}$ & -0.30 & $\begin{array}{l}+0.03 \\
-0.03\end{array}$ & 0.339 & 0.059 & 0.62 & 0.45 & 0.48 \\
\hline 110.9 & $\begin{array}{l}+2.0 \\
-1.6\end{array}$ & -0.36 & $\begin{array}{l}+0.03 \\
-0.03\end{array}$ & 0.515 & 0.083 & 0.82 & 0.61 & 0.70 \\
\hline 138.7 & $\begin{array}{l}+1.5 \\
-2.0\end{array}$ & -0.75 & $\begin{array}{l}+0.02 \\
-0.02\end{array}$ & 0.133 & 0.046 & 0.12 & 0.03 & $0=11$ \\
\hline 142.0 & $\begin{array}{l}+2.1 \\
-1.8\end{array}$ & -0.79 & $\begin{array}{l}+0.02 \\
-0.02\end{array}$ & 0.114 & 0.038 & 0.07 & 0.00 & 0.07 \\
\hline
\end{tabular}


Table 3.116 ;

Same as table 3.11(a) except that bins have not been divided.

\begin{tabular}{|c|c|c|c|c|c|c|c|c|}
\hline$\theta_{\mathrm{cm}}$ & $\Delta^{\theta} \mathrm{crn}$ & $\cos \theta \mathrm{crn}$ & $\Delta \cos \theta_{\mathrm{Cn}}$ & $\mathrm{A}_{\mathrm{N}}$ & $\sigma_{A_{I N}}^{-}$ & $\mathrm{K}-\mathrm{H}$ & CMU-LBL & VPI \\
\hline 54.4 & $\begin{array}{l}+7.3 \\
-8.1\end{array}$ & D. .58 & $\begin{array}{l}+0.11 \\
-0.11\end{array}$ & -0.862 & 0.027 & -0.79 & -0.87 & -0.90 \\
\hline 68.2 & $\begin{array}{l}+6.1 \\
-6.5\end{array}$ & 0.37 & $\begin{array}{l}+0.10 \\
-0.10\end{array}$ & -0.833 & 0.013 & -0.85 & -0.91 & $=0.93$ \\
\hline 81.9 & $\begin{array}{l}+5.2 \\
-6.6\end{array}$ & 0.14 & $\begin{array}{l}+0.11 \\
-0.09\end{array}$ & -2.680 & $0.0 \% 4$ & -0.81 & -0.82 & -0.86 \\
\hline 103.1 & $\begin{array}{l}+3.8 \\
-3.4\end{array}$ & -0.33 & $\begin{array}{l}+0.06 \\
-0.06\end{array}$ & 0.404 & 0.049 & 0.72 & 0.54 & 0.61 \\
\hline 140.6 & $\begin{array}{l}+3.5 \\
-3.9\end{array}$ & -0.77 & $\begin{array}{l}+0.04 \\
-0.174\end{array}$ & 0.129 & 0.031 & 0.09 & 0.01 & 0.08 \\
\hline
\end{tabular}


Table 3.12(a)

Results of the measurement of the asymmetry parameter in $\pi^{-}$p elastic scattering at $687 \mathrm{MeV} / \mathrm{c}$ together with phase shift analysis predictions. only statistical errors are listed. The systematical error due to the uncertainty in the absolute target polarization is $\pm 3 \%$.

\begin{tabular}{|c|c|c|c|c|c|c|c|c|}
\hline$\theta_{\mathrm{cm}}$ & $\Delta \theta_{\mathrm{cm}}$ & $\cos { }_{\mathrm{cm}}$ & $\Delta \cos \theta_{\mathrm{cm}}$ & $\mathrm{A}_{\mathrm{N}}$ & $\sigma_{A_{N}}^{-}$ & $\mathrm{K}-\mathrm{H}$ & CMU-LBL & VPI \\
\hline 47.6 & $\begin{array}{l}+1.1 \\
-2.8\end{array}$ & 0.67 & $\begin{array}{l}+0.04 \\
-0.01\end{array}$ & -0.495 & 0.056 & -0.53 & -0.52 & -0.62 \\
\hline 50.6 & $\begin{array}{l}+1.7 \\
-1.9\end{array}$ & 0.63 & $\begin{array}{l}+0.03 \\
-0.02\end{array}$ & -0.532 & 0.030 & -0.57 & -0.55 & -0.65 \\
\hline 53.9 & $\begin{array}{l}+2.2 \\
-1.6\end{array}$ & 0.59 & $\begin{array}{l}+0.02 \\
-0.03\end{array}$ & -0.567 & 0.049 & -0.61 & -0.58 & -0.68 \\
\hline $63.7^{\star}$ & $\begin{array}{l}+1.9 \\
-1.5\end{array}$ & 0.44 & $\begin{array}{l}+0.03 \\
-0.02\end{array}$ & $\cdots 0.680$ & 0.047 & -0.70 & -0.66 & -0.75 \\
\hline $66.9^{*}$ & $\begin{array}{l}+1.7 \\
-2.0\end{array}$ & 0.39 & $\begin{array}{l}+0.03 \\
-0.03\end{array}$ & -0.717 & 0.039 & -0.72 & -0.67 & -0.77 \\
\hline $79.6^{*}$ & $\begin{array}{l}+1.6 \\
-1.2\end{array}$ & 0.18 & $\begin{array}{l}+0.03 \\
-0.02\end{array}$ & -0.811 & 0.085 & -0.75 & -0.63 & -0.76 \\
\hline $82.1^{*}$ & $\begin{array}{l}+1.3 \\
-1.6 \\
+1.6\end{array}$ & 0.14 & $\begin{array}{l}+0.02 \\
-0.03 \\
+0.04\end{array}$ & -0.791 & 0.078 & -0.74 & -0.59 & -0.73 \\
\hline 108.1 & $\begin{array}{l}+1.0 \\
-2.4\end{array}$ & -0.31 & $\begin{array}{l}+0.04 \\
-0.03\end{array}$ & 0.311 & 0.077 & 0.55 & 0.48 & 0.47 \\
\hline 111.4 & $\begin{array}{l}+2 . ! \\
-1.7 \\
+1.7\end{array}$ & -0.36 & $\begin{array}{l}+0.02 \\
-0.04\end{array}$ & 0.045 & 0.054 & 0.30 & 0.16 & 0.23 \\
\hline 140.3 & $\begin{array}{l}+1.7 \\
-2.0 \\
+2.0\end{array}$ & -0.77 & $\begin{array}{l}+0.02 \\
-0.02 \\
+0.01\end{array}$ & -0.298 & 0.048 & -0.47 & -0.53 & -0.48 \\
\hline 143.5 & $\begin{array}{l}+2.0 \\
-1.5\end{array}$ & -0.80 & $\begin{array}{l}+0.01 \\
-0.02\end{array}$ & -0.269 & 0.058 & -0.47 & -0.51 & -0.4 \\
\hline 160.5 & $\begin{array}{l}+3.8 \\
-3.2\end{array}$ & -0.94 & $\begin{array}{l}+0.02 \\
-0.02\end{array}$ & -0.170 & 0.036 & -0.33 & -0.32 & -0.3 \\
\hline
\end{tabular}

* Protons were detected in the LAS. 
Table $3.12(b)$

Same as table 3.12(a) except that bins have not been divided.

\begin{tabular}{|c|c|c|c|c|c|c|c|c|}
\hline$\theta_{\mathrm{cra}}$ & $\Delta \theta_{\mathrm{cII}}$ & $\cos ^{9} \mathrm{crn}$ & $\Delta \cos \theta_{\mathrm{cm}}$ & $A_{N}$ & $\sigma_{\mathrm{A}_{\mathrm{N}}}^{-}$ & $\mathrm{K}-\mathrm{H}$ & CMU-LBL & VPI \\
\hline 51.4 & $\begin{array}{l}+4.7 \\
-6.6\end{array}$ & 0.62 & $\begin{array}{l}+0.09 \\
-0.06\end{array}$ & -0.547 & 0.025 & -0.58 & -0.56 & -0.66 \\
\hline $65.5^{*}$ & $\begin{array}{l}+3.8 \\
-3.3\end{array}$ & 0.41 & $\begin{array}{l}+0.06 \\
-0.05\end{array}$ & -0.705 & 0.030 & -0.71 & -0.66 & -0.76 \\
\hline $81.0^{*}$ & $\begin{array}{l}+3.0 \\
-2.7\end{array}$ & 0.16 & $\begin{array}{l}+0.05 \\
-0.05\end{array}$ & -0.805 & 0.059 & -0.75 & -0.61 & -0.74 \\
\hline 109.9 & $\begin{array}{l}+3.6 \\
-4.2\end{array}$ & -0.34 & $\begin{array}{l}+0.07 \\
-0.06\end{array}$ & 0.163 & 0.044 & 0.42 & 0.30 & 0.37 \\
\hline 141.8 & $\begin{array}{l}+3.7 \\
-3.5\end{array}$ & -0.79 & $\begin{array}{l}+0.04 \\
-0.03\end{array}$ & -0.296 & 0.038 & -0.47 & -0.52 & -0.47 \\
\hline 160.5 & $\begin{array}{l}+3.8 \\
-3.2\end{array}$ & -0.94 & $\begin{array}{l}+0.02 \\
-0.02\end{array}$ & -0.170 & 0.036 & -0.33 & -0.32 & -0.30 \\
\hline
\end{tabular}

*Protons were detected in the LAS. 


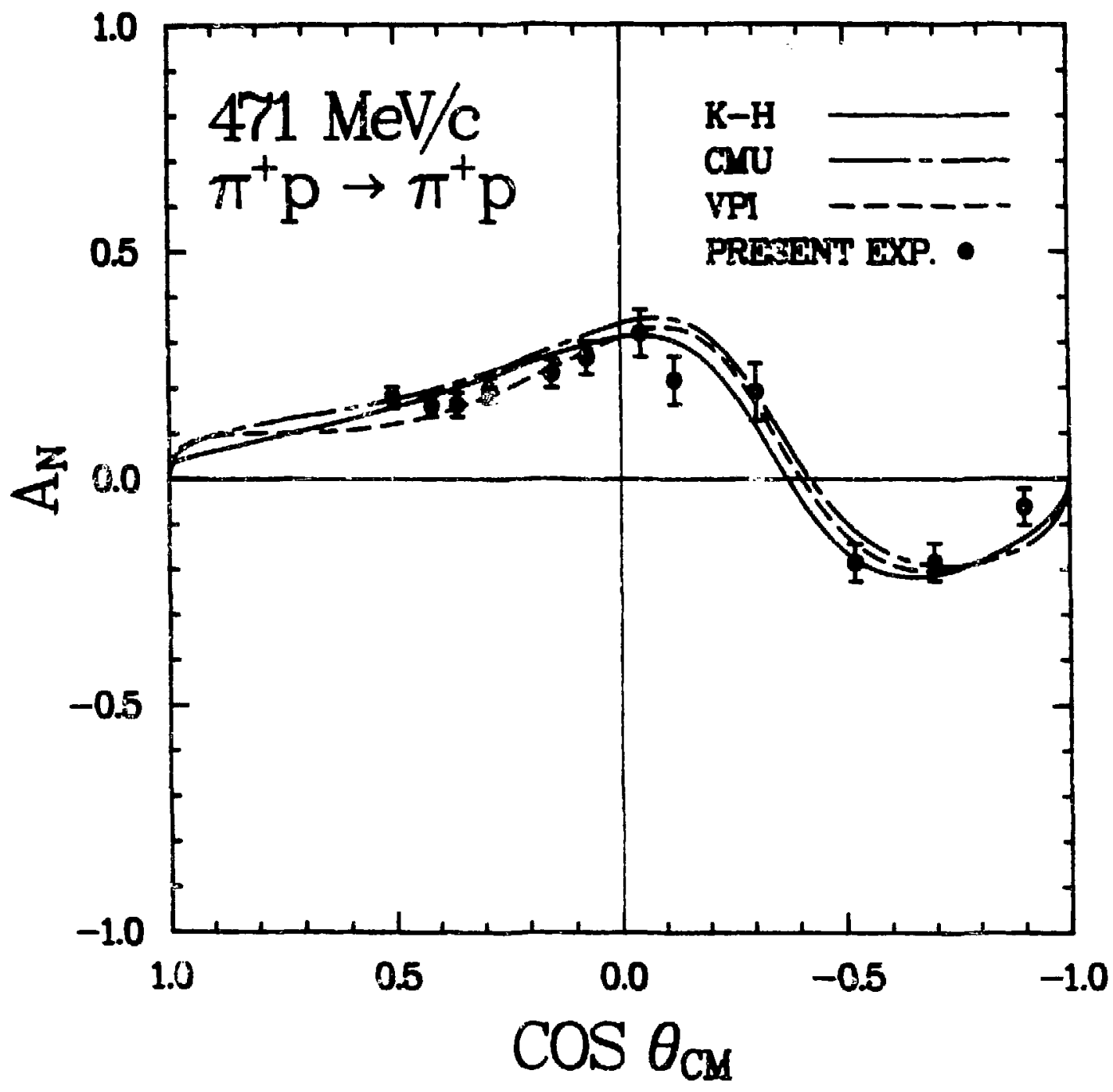

Figure 3.14. Asymmetry parameter for $\pi^{+} p$ elastic scattering at $47 \mathrm{l} \mathrm{MeV} / \mathrm{c}$ together with the PWA predictions. Solid curve shows the K-H phase shift analysis predictions. The chain-dashed curve presents the CMU-LBL partial wave analysis results. The dashed curve exhjbits the VPI solution. 


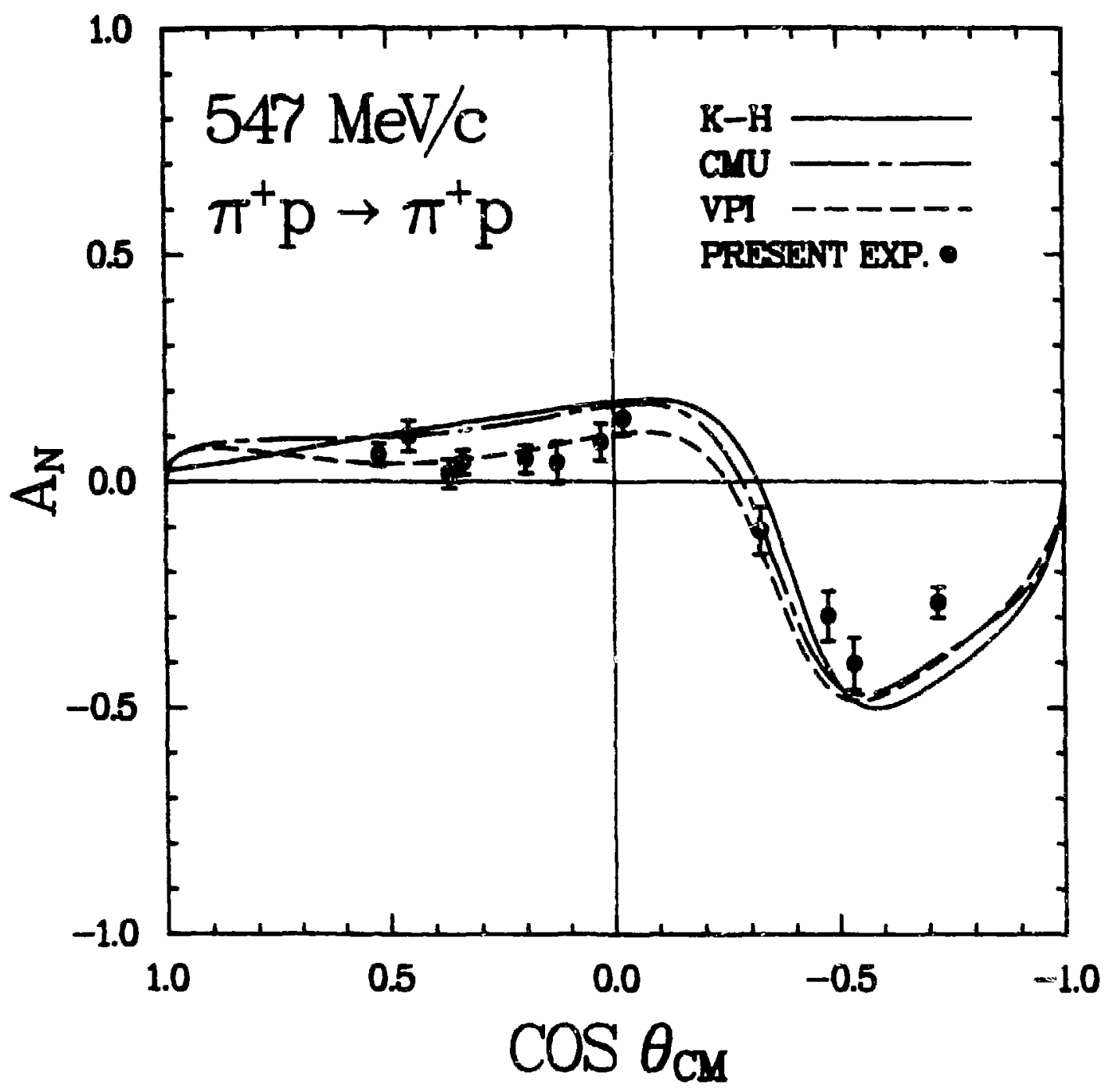

Figure 3.15. Asymetry parameter for $\pi^{+} \mathrm{p}$ elastic scattering at $547 \mathrm{MeV} / \mathrm{c}$ together with the PWA predictions. Solid curve shows the $\mathrm{K}-\mathrm{H}$ phase shift analysis predictions. The chain-dashed curve presents the CMU-LBL partial wave analysis results. The dashed curve exhibits the VPI solution. 


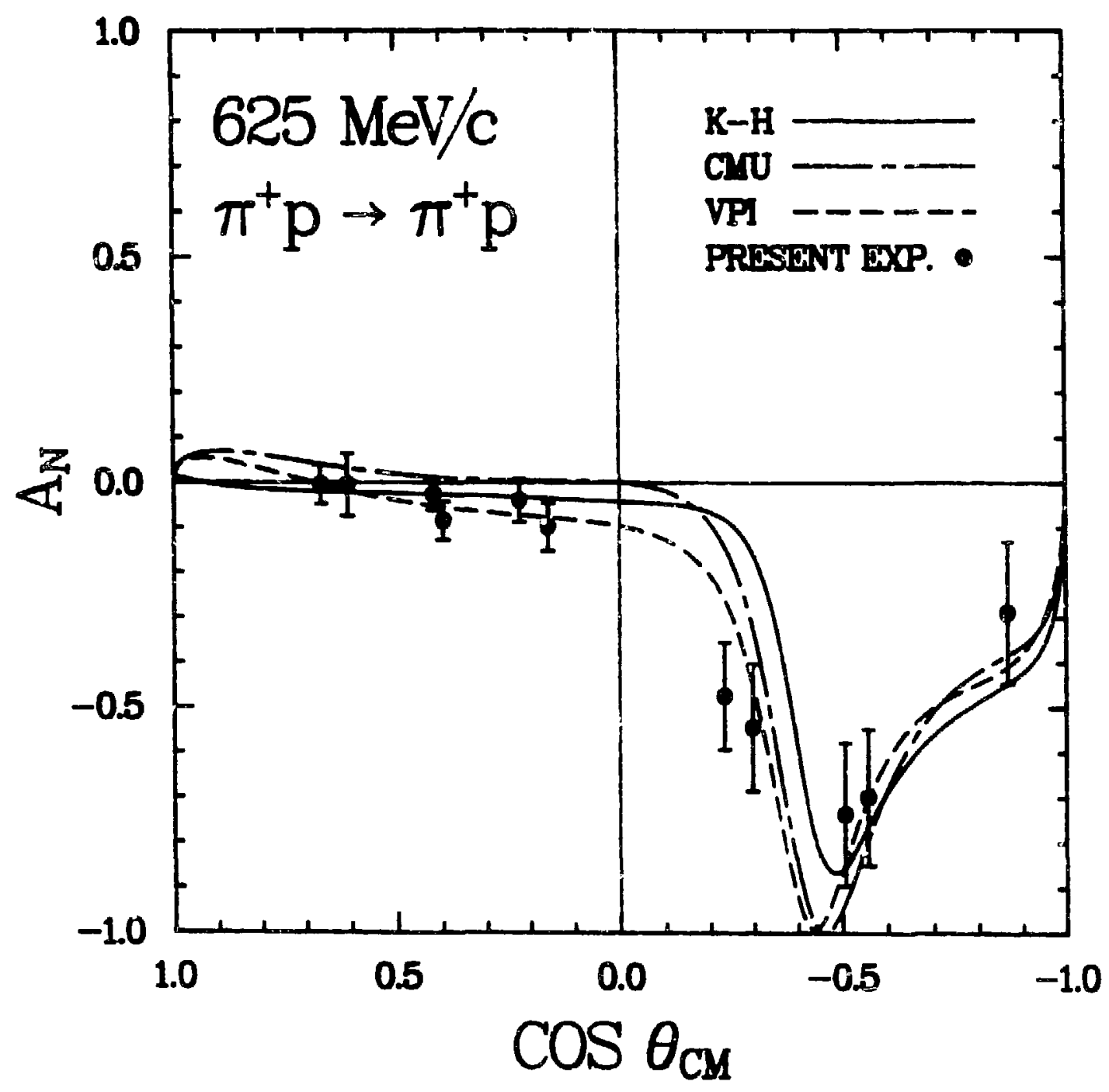

Figure 3.16. Asymetry parameter for $\pi^{+} \mathrm{p}$ elastic scatcering at $625 \mathrm{MeV} / \mathrm{c}$ together with the PWA predictions. Solid curve shows the $\mathrm{K}-\mathrm{H}$ phase shift analysis predictions. The chain-dashed curve presents the CMU-LBL partial wave analysis results. The dashed curve exhibits the VPI solution. 


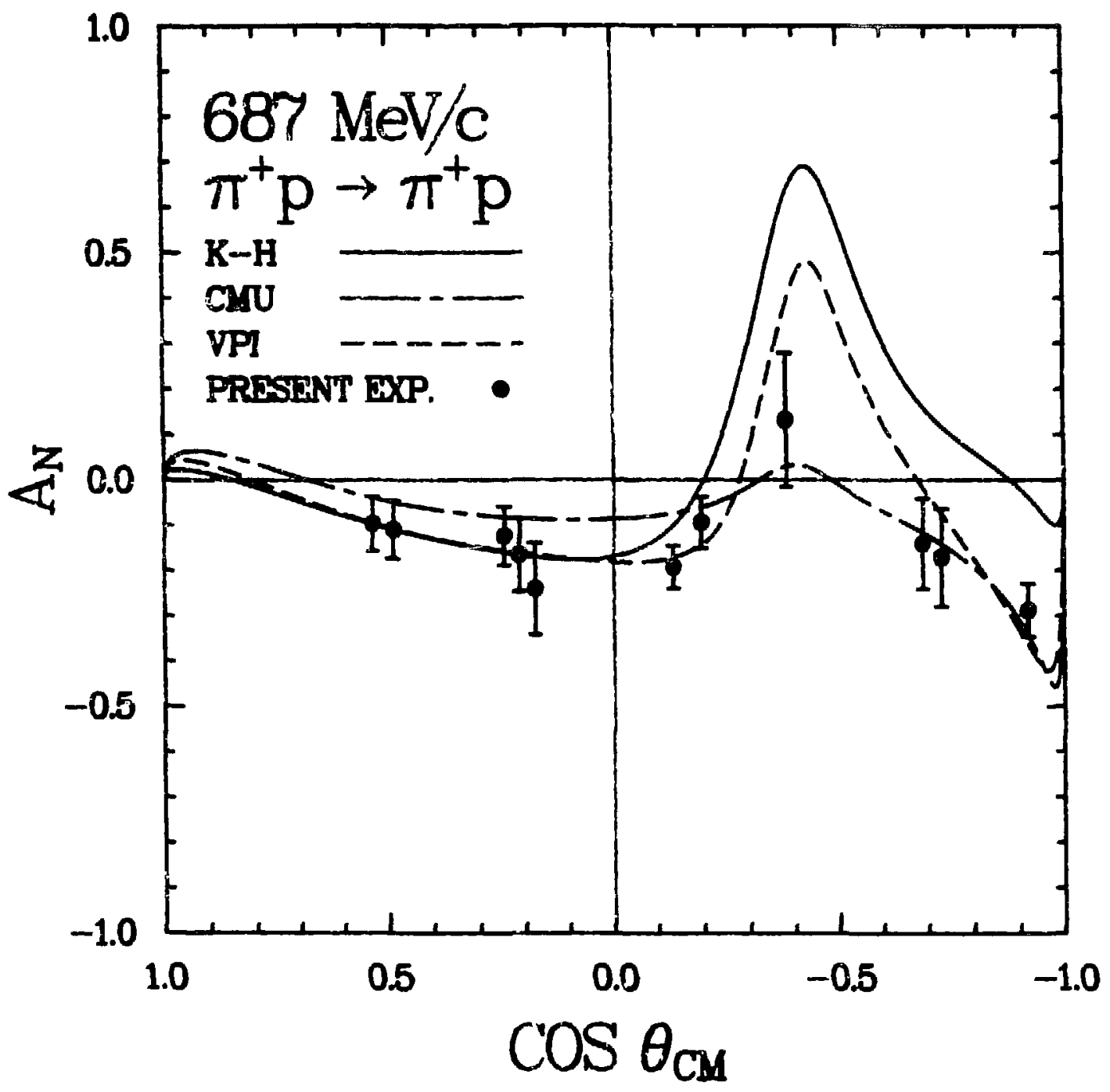

Figure 3.17. Asymmetry parameter for $\pi^{+} \mathrm{p}$ elastic scattering at $687 \mathrm{M} \in \mathrm{l} / \mathrm{C}$ together with the PWA predictions. Solid curve shows the $\mathrm{K}-\mathrm{H}$ phase shift analysis predictions. The chain-dashed curve presents the CMU-LBL partial wave analysis resilts. The dashed curve exhibits the VPI solution. 


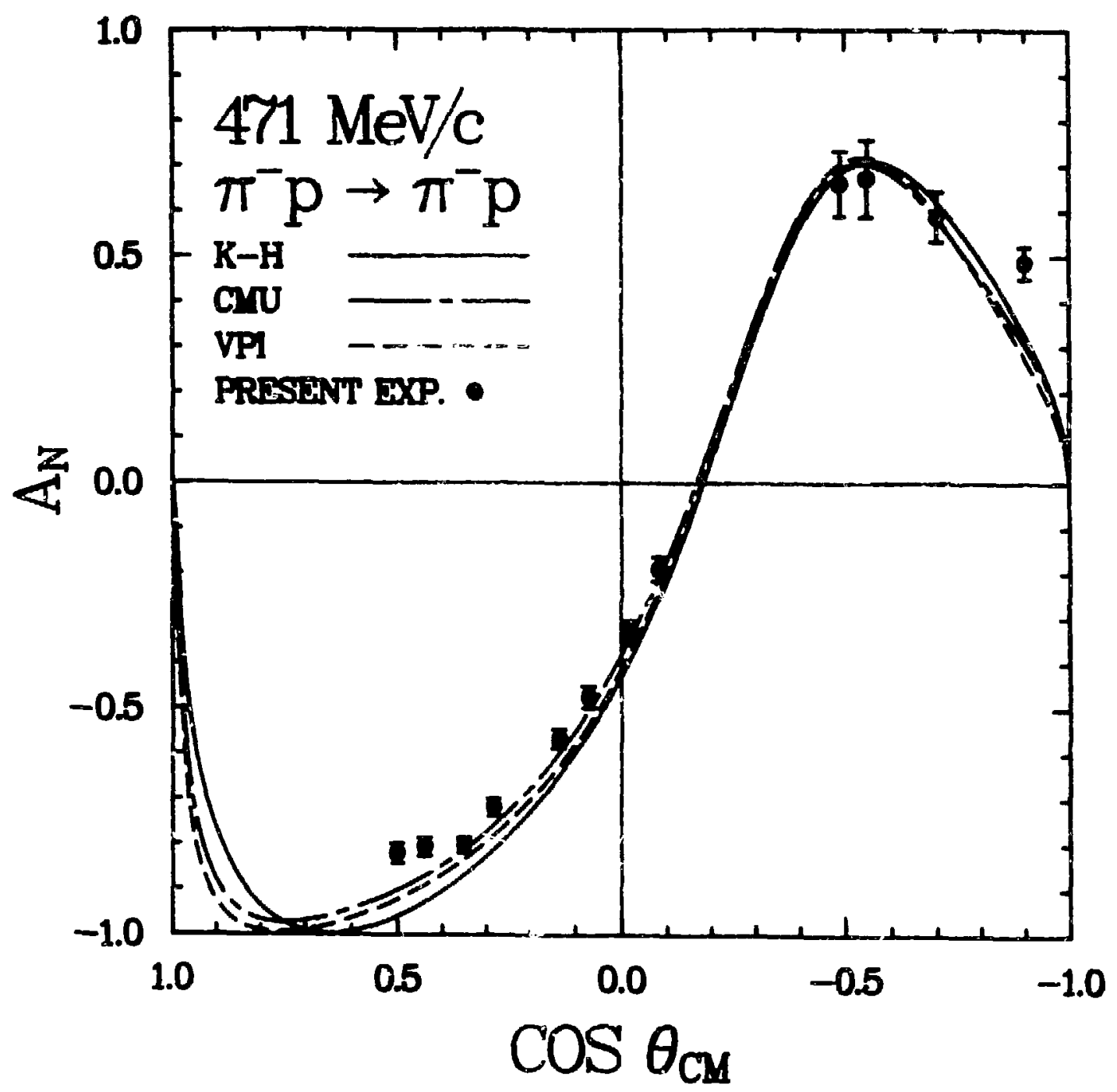

Figure 3.18. Asymetry parameter for $\pi^{-p}$ elastic scattering at $471 \mathrm{MeV} / \mathrm{C}$ together with the PWA predictions. Solid curve shows the $\mathrm{K}-\mathrm{H}$ phase shift analysis predictions. The chain-dashed curve presents the CMU-LBL partiai wave analysis results. The dashed curve exhibits the VPI solution. 


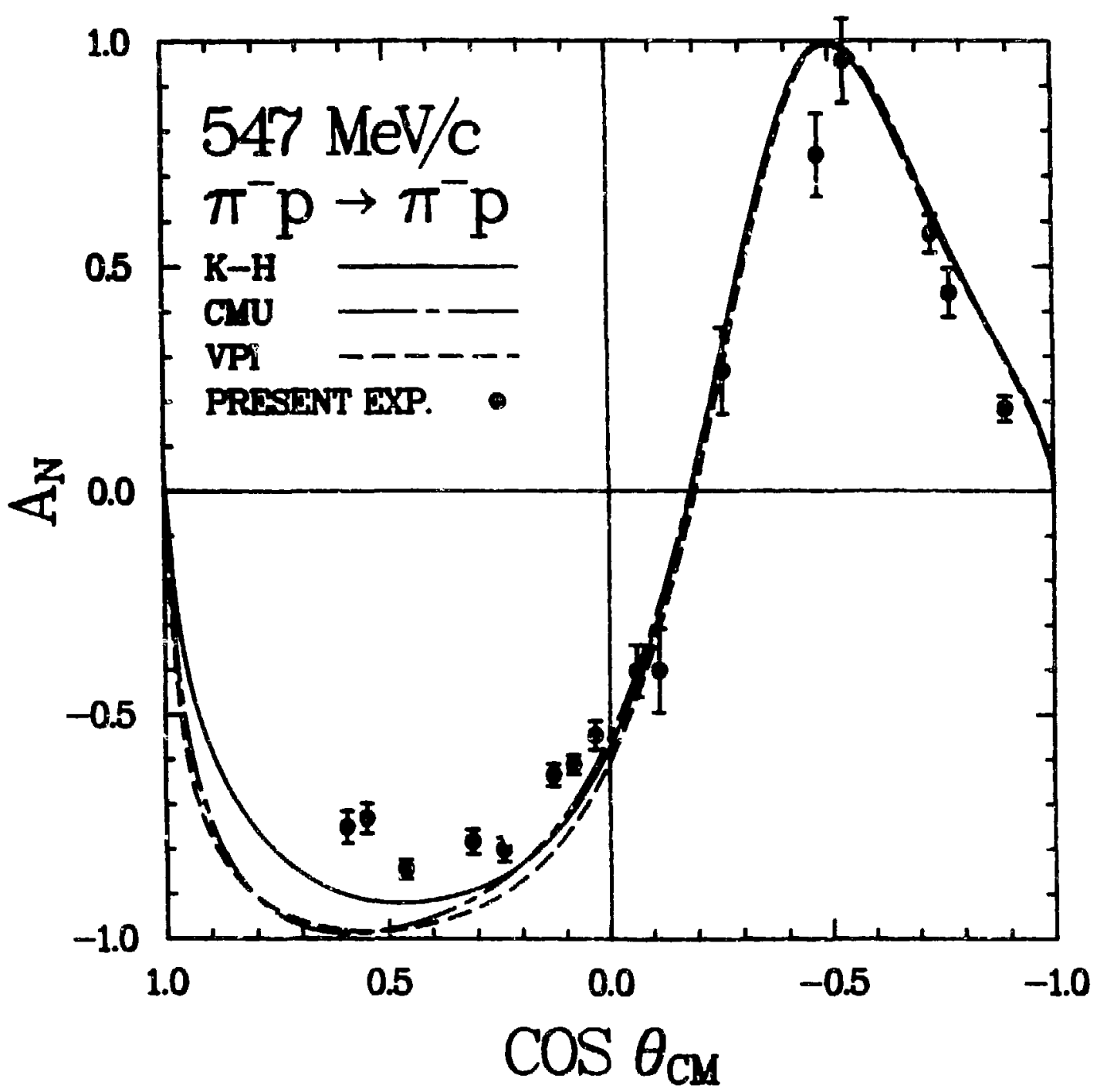

Figure 3.19. Asymetry parameter for $\pi^{-} \mathrm{p}$ elastlc scattering at $547 \mathrm{MEV} / \mathrm{c}$ together with the PWA predictions. Solid curve shows the $\mathrm{K}-\mathrm{H}$ phase shift analysis predictions. The chain-dashed curve presents the CMU-LBL partial wave analysis results. The dashed curve exhibits the VPI soiution. 


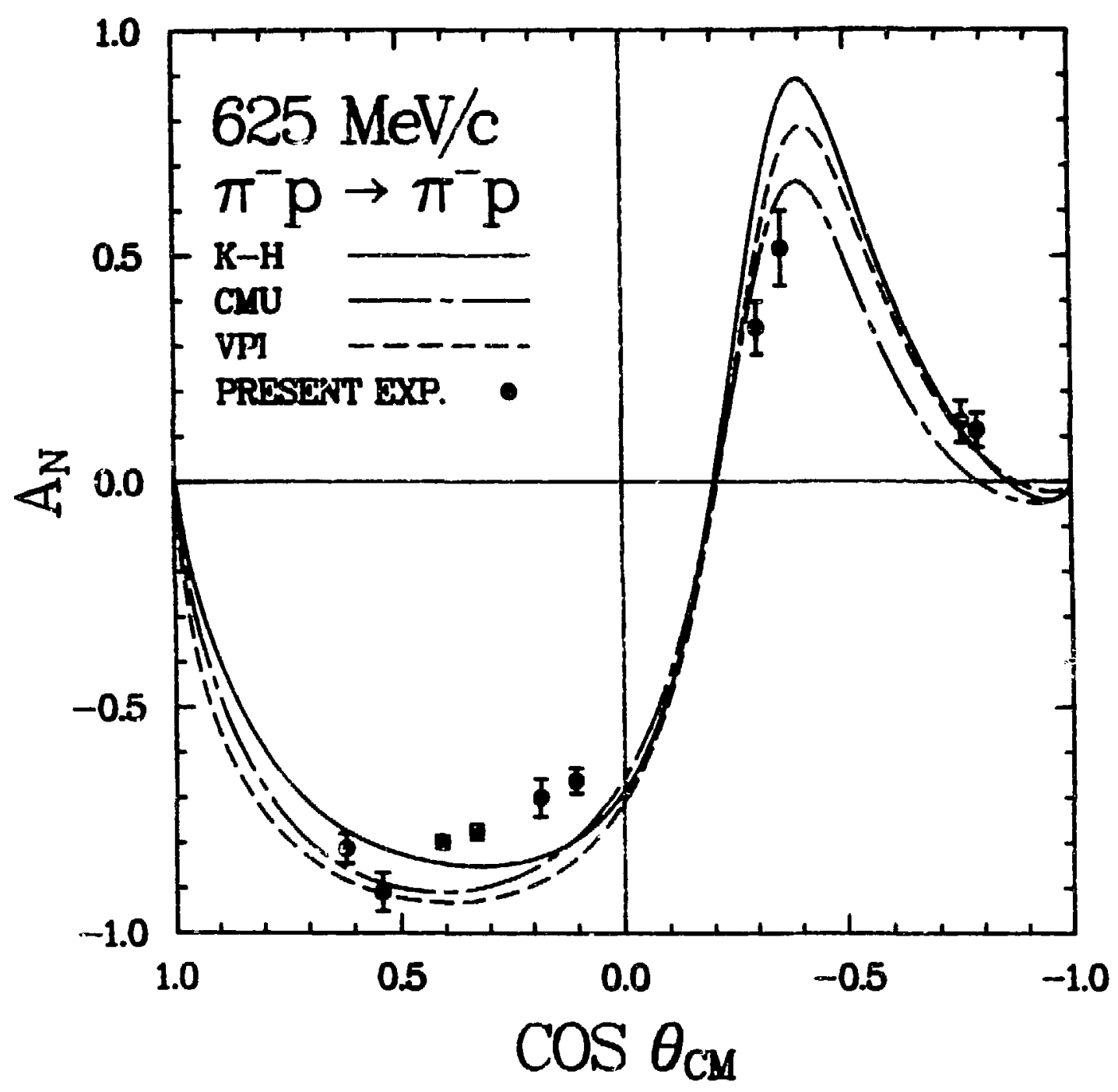

Figure 3.20. Asymetry parameter for $\pi^{-} \mathrm{p}$ elastic scattering at $625 \mathrm{MeV} / \mathrm{c}$ together with the PWA predictions. Solid curve shows $t \mathrm{H}: \mathrm{K}-\mathrm{H}$ phase shift analysis predictions. The chain-dashed curve presents the CMU-LBL partial wave analysis results. The dashed curve exhibits the VRI solution. 


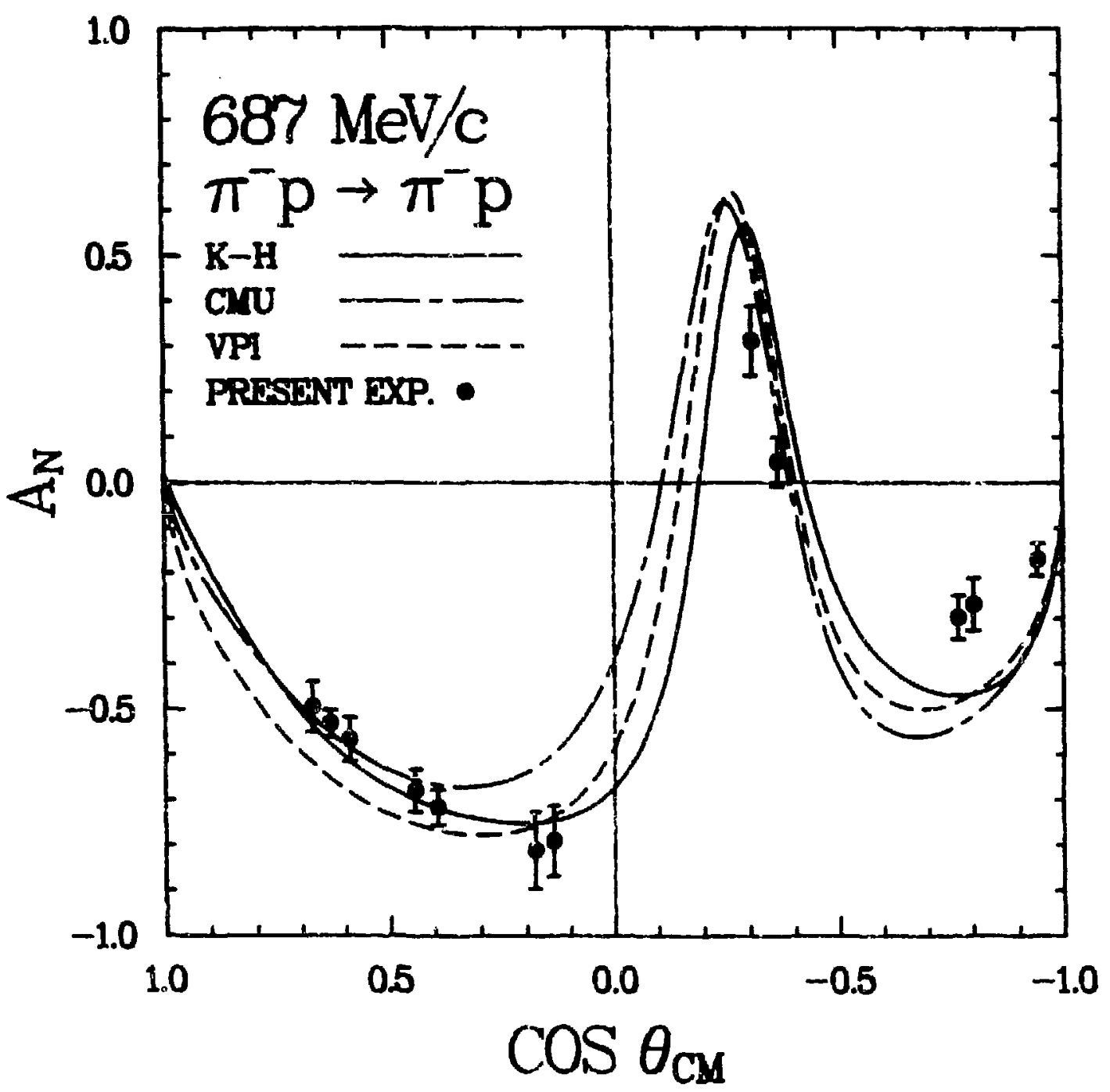

Figure 3.21. Asymetry parameter for $\pi^{-} p$ elastic scattering at $687 \mathrm{MeV} / \mathrm{c}$ together with the PWA predictions. Solid curve shows the K-H phase shift analysis predictions. The chain-dashed curve prasents the CMU-LBL partial wave analysis results. The dashed curve exhibits the VPI solution. 


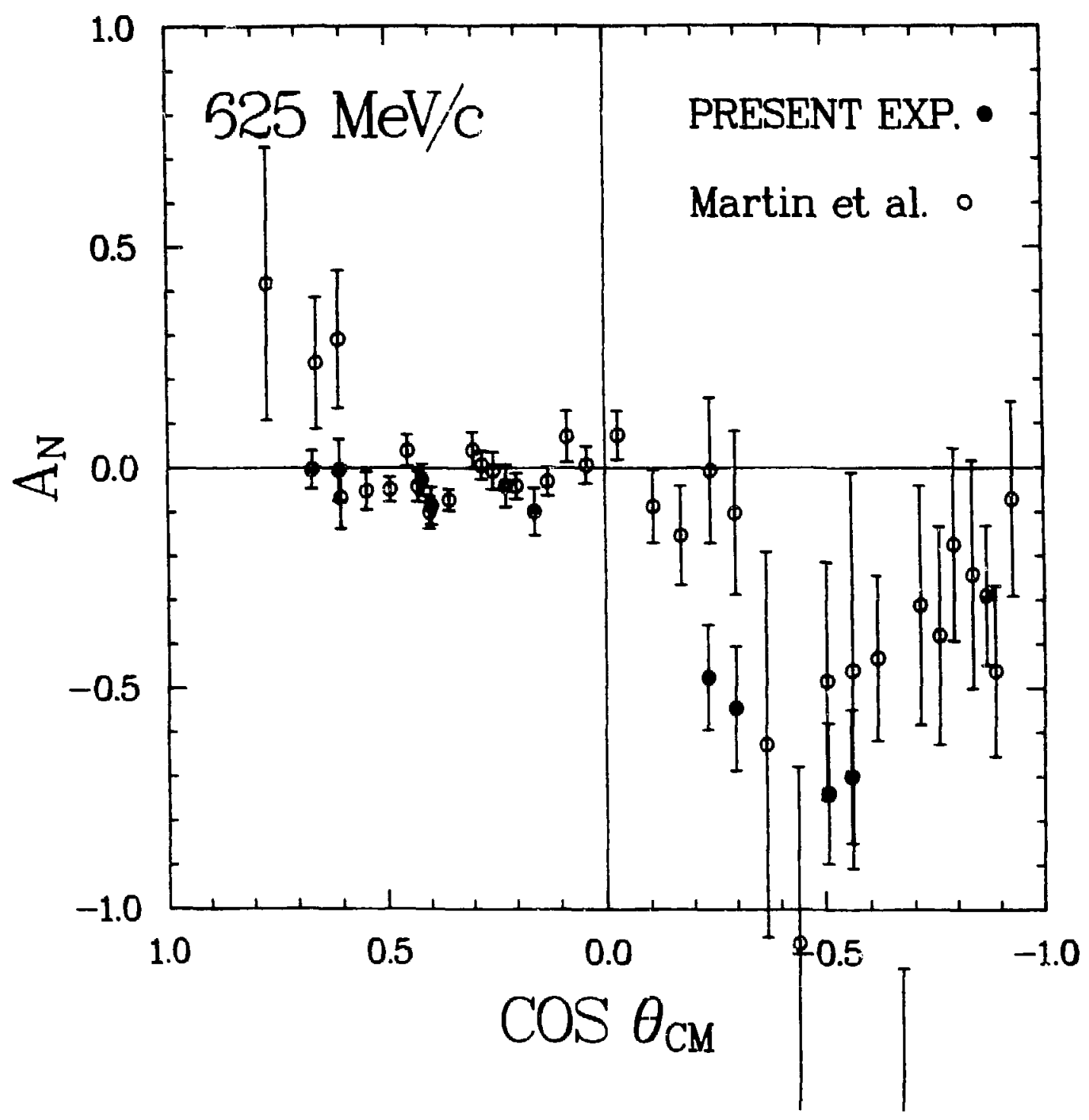

Figure 3.22 . Comparison of our $\pi^{+}$p results at $625 \mathrm{MeV} / \mathrm{C}$ with Martin et al data at $617 \mathrm{MeV} / \mathrm{c}$. 


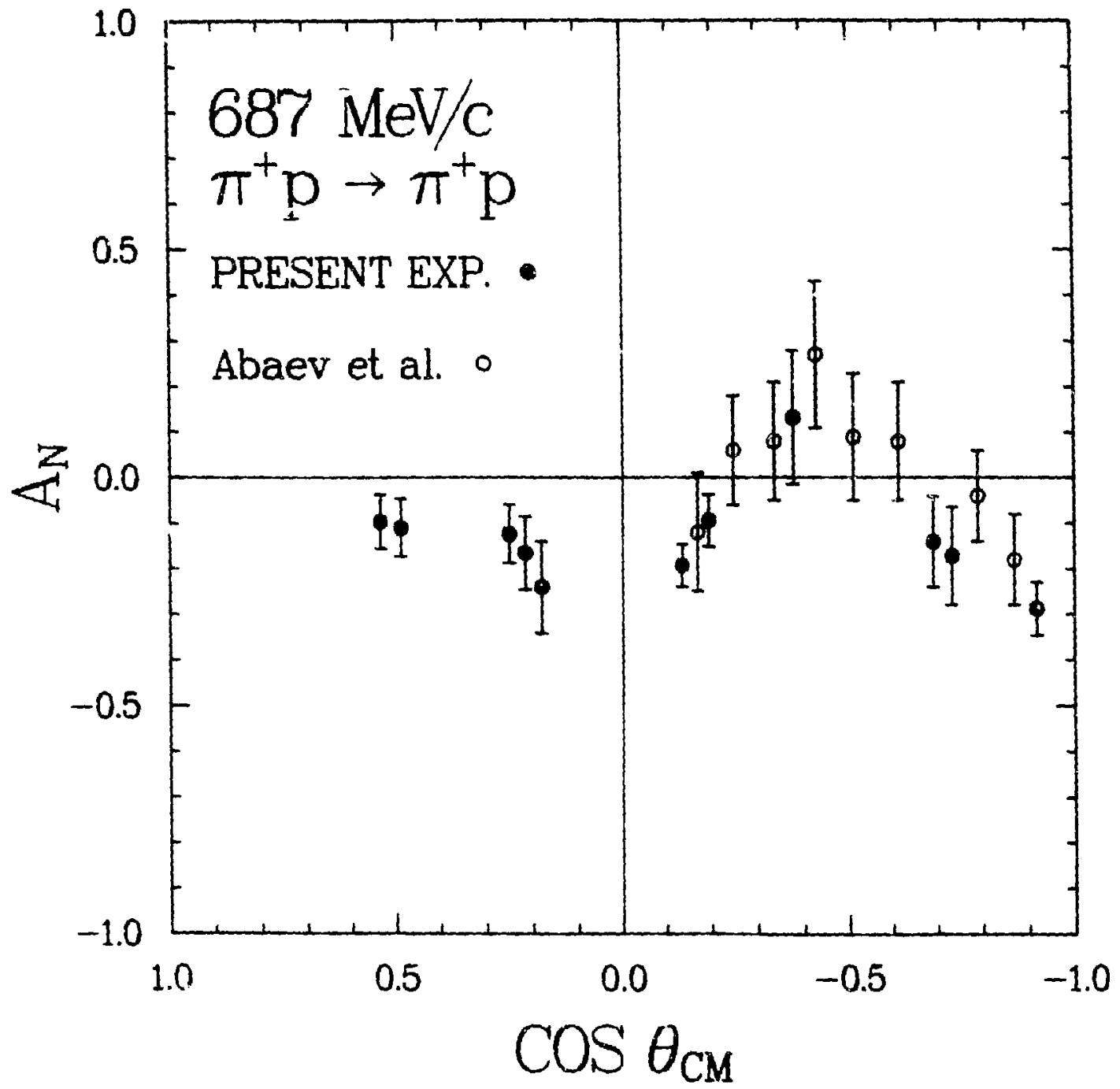

Figure 3.23. Our $\pi^{+} p$ results at $687 \mathrm{MeV} / \mathrm{C}$ compared to Abaev et al. data at $685 \mathrm{MeV} / \mathrm{C}$. 


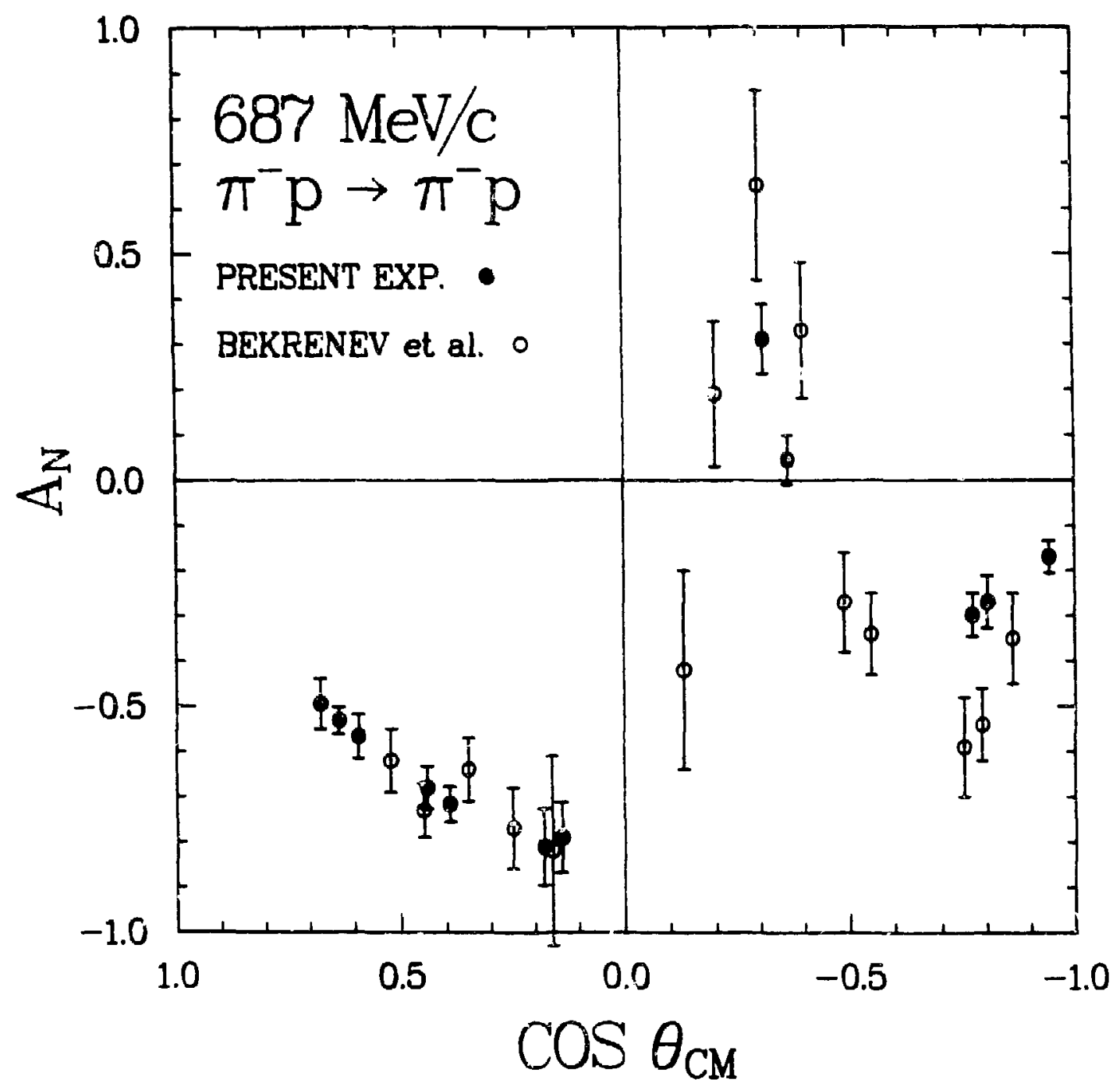

Figure 3.24. Comparison of our $\pi^{-}$p results at $687 \mathrm{MeV} / \mathrm{c}$ with Bekrenev et al. data at $685 \mathrm{MeV} / \mathrm{c}$. 


\section{Acknowledgements}

I an most grateful to Professor Bernard M. K. Nefkens for his guidance and advice throughout all stages of this work. I am also grateful to Dr. Daniel H. Fitzgerald who was largely responsible for the design, setup, for proofreading the original manuscript, and spent much time discussing the experiment. It is a pleasure to thank Dr. William J. Briscoe who also heiped to setup the experiment, data acquisition, and fitting program. I would like to thank $\mathrm{Dr}$. Michael E. Sadler who helped with the data acquisition, fitting routines and phase shift program. Special thanks go to Dr. Peter Gram who helped with the LAS spectrometer and the staff of LAMPF for their efforts in making this work possible. I am very grateful to Dr. J.E. Simmons at Los Alamos National Laboratory P-division for the loan of the polarized target and the extensive help of J.G.J. Boissevain in the initial setup of the target. I would like to thank Dr. George Glass who patiently explained tracing of charged particles in the field of polarized target magnet to me. I would also like to thank W.B. Tippens, J. Holt, G.J. Kim, A.D. Eichon, and J.A. Wightman. Finally, I would like to thank Mrs. Barbara Cabot and Mrs. Pamela Polin for their kind assistance during my graduate studies at UCLA, and Mrs. Maggie Eutsler at LAMPF。 


\section{REFERENCES}

$l_{W}$. Panofsky, Proceedings of International Symposium on High Energy Physics, Vienna, 1968.

2M. Gell-Mann, Phys, Lett. 8 , 214 (1964).

${ }^{3}$ G. Zweig, CERN Preprint TH412 (1964) unpublished.

${ }^{4}$ N. Isgur and G. Karl, Phys. Rev. D18, 4187 (1978); ibid. D19, 2653

(1979); ibid. D20, 1191 (1979); ibid. D21, 1868 (1980).

${ }^{5}$ G.H. Höhler et a1., Handbook of Pion-Nucleon Scattering, Physics Data 12-1, Kar1sruhe, Germany (1979).

${ }^{6}$ R.E. Cutkosky et al., Fhys. Rev. D20, 2804 (1979).

${ }^{7}$ R. Koch and E. Pietarinen, Nucl. Phys. A336, 331 (1980).

${ }^{8}$ V.S. Zidel1, R.A. Arndt and L.D. Roper, Phys. Rev. D21, 1225 (1980).

9 V.A. Gordeev et a1., Nucl. Phys. A364, 408 (1981).

$10 \mathrm{R}$. Ayed and P. Bareyre, Proc. Aix-en-Provence Conf. (1973).

${ }^{11} \mathrm{G}$. Karl, Proceedings of Second LAMPF II Workshop; LA-9572-C, 197 (1982).

${ }^{12}$ V.S. Bekrenev et a1., Nucl. Phys. A364, 515 (1981).

13 P.L. Csonka and M.J. Moravscik, Phys. Rev. 152, 1310 (1966).

14 J.J. Sakurai, Lnvariance Principles and Elementary Particles (Princeton University Press, Princeton, 1964).

15 W.S.C. Williams, An Introduction to Elementary Particles (Academic Press, New York, 1961).

${ }^{16} \mathrm{~L}$. Wolfenstein and J. Ashkin, Phys. Rev. $\underline{85}, 947$ (1952).

17R.H. Dalitz, Proc. Phys. Soc. A65, 175 (1952).

18 J.S. Bell and F. Mandl, Proc. Phys. Soc. A7l, 273 (1958);

Proc. Phys. Soc. A71, 867 (1958).

${ }^{19}$ U. Fano, Rev. Mod. Phys. 29, 74 (1957).

20 M.L. Goldberger and K.M. Watson, Collision Theory (John Wiley and Sons, New York, 1964 ). 


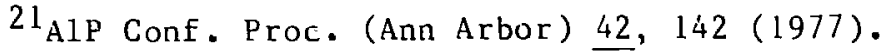

${ }^{22}$ G. Källén, Elementary Particle Physics (Academic Press, Reading, Mass, 1964).

${ }^{23}$ N.F. Mott and H.S.W. Massey, The Theory of Atomic Collisions (Clarendon Press, Oxford, 1968).

24 S. Minami, Progr. Theoret. Phys. 11,213 (1954).

${ }^{25}$ N.W. Dean and Ping Lee, Phys. Rev. D5, 2741 (1972).

$26 \mathrm{~J}$. Orear, Phys. Rev. 96, 1417 (1954).

$27 \mathrm{P}^{3}$ User Group and P.A.M. Gram, A Proposal Eor $\mathrm{P}^{3}$, A Versatile High-Energy Pion Beam Facility, LASL Report 非A-4535-MS, 1970.

28 M.S. Livingston, LASL Report LA-5000, NM (1972).

${ }^{29}$ H.H. Howard, B. Storms and S.P. Slatkin, LAMPF Ustrs Handbook, LASL, Medium Energy Physics Division, Los Alamos, NM (1974).

${ }^{30}$ W.J. Briscoe et. al., $\mathrm{P}^{3}$ Channel Beam Measurements at LAMPF, LASL Report LA-UR 81-2537.

${ }^{31}$ K.l. Brown, F. Rothacker, D.C. Carey and Ch. Iselin, SLAC-91, Rev. 2 (1977); CERN Report, CERN 73-16 (1973).

${ }^{32}$ G..I. Krausse and P.A.M. Gram, Nucl. Instr. and Meth. 156,365 (1978).

33. W. Tippens, Los Alamos Keport LA-9909-T (1983).

34 Polarized Proton Target Handbook, Los Alamos PPT I (1977).

${ }^{35} \mathrm{~A}$. Abragam, The Principles of Nuclear Magnetism, Oxford University Press, Amen House, London E.C.4 (1973).

36. Abragam and M. Goldman, Rep. on Progr. Phys. 41, 395 (1978).

37 M. Borghini, Proc. 2nd Int. Conf. on Polarized Targets, Ed. G. Shapiro, Berkeley, LBL 500, 1 (1978).

38 S.G. Sydoriak and T.R. Roberts, Phys. Rev. 106, 175 (1957).

39w. Boer, Nucl. Instr. and Meth. 107, 99 (1973).

$40 \mathrm{E}$. Colton, Nucl. Instr. and Meth. 178, 95 (1980).

41 Manufactured by LeCroy P.esearch Systems Corporation, Spring Valley, N.Y. 10977.

42J. Faucett, Los Alamos Report LA-9970-T (1984).

${ }^{43}$ L.R. Biswell and R.E. Rajala, LASL Report, LA-5144 (1973).

44 M. Kellogg et al., Introduction to Q, LASL Report, LA-7001-M (1978). 45 R.A. Arndt, Private Communication, August 1984. 
${ }^{46} \mathrm{C}$. Amsler et al., Phys. Lett. 57B, 289 (1975).

47 J.C. Alder et al., Nuovc Cim. Lett. A300, 321 (1978).

48.G. Albrow et al., Nucl. Phys. B25, 9 (1971); B37, 594 (1972).

49 J.F. Martin et al., Nucl. Phys. B89, 253 (1975).

50 V.V. Abaev et al., Z. Phys. A311, 217 (1983).

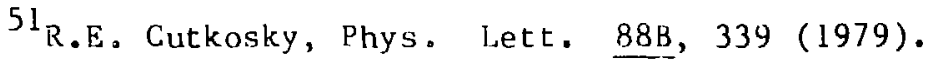


Appendix A. Beam line shunt readings.

$\frac{47 \mathrm{MeV} / \mathrm{c}}{\pi^{-}} \quad \frac{547 \mathrm{MeV} / \mathrm{c}}{\mathrm{if}^{+}} \quad \frac{625 \mathrm{MeV} / \mathrm{c}}{\mathrm{if}^{+}} \quad \frac{687 \mathrm{MeV} / \mathrm{c}}{\mathrm{ir}^{-}}$

\begin{tabular}{|c|c|c|c|c|c|c|c|c|c|}
\hline & & & & & & & & & \\
\hline 102 & $\mathrm{aV})$ & & & & & & & & \\
\hline 01 & & & & & & & & & \\
\hline & & & & & & & & & \\
\hline & & & & & & & & & \\
\hline & & & & & & & & & \\
\hline & & & & & & & & & \\
\hline & & & & & & & & & \\
\hline 107 & & & & & & & & & \\
\hline 408 & & & & & & & & & \\
\hline 103 & & & & & & & & & \\
\hline 11 & & & & & & & & & \\
\hline MI & ) & & & & & & & & \\
\hline & V) & & & & & & & & \\
\hline & & & & & & & & & \\
\hline 8 & $(0-)^{*}$ & & & & & & & & +99 \\
\hline 502 & & & & & & & & & 63 \\
\hline & & & & & & & & & 9 \\
\hline $4 \mathrm{~S} 3 \mathrm{~W}$ & $(\mathrm{~cm})$ & & & & 0.401 & & & & 99 \\
\hline MS 04 & $(\mathrm{~cm})$ & & 10.090 & 7.161 & 4.897 & & 12.2 & & 15.437 \\
\hline & $(\mathrm{cm})$ & & 15.375 & 4.5 & 8.951 & & & & 18.23 \\
\hline EAB: & $(\mathrm{cm})$ & & 1.002 & 10.8 & 1.004 & 10.7 & 0.766 & & 0.72 \\
\hline$P A B$ & & & 1.010 & & & & 0.654 & & 0.63 \\
\hline & & & -0.389 & & & -0.393 & -0.373 & -0 & -0.39 \\
\hline 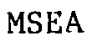 & ) & & 19.241 & & & 19.236 & 6.246 & 01 & 17.58 \\
\hline
\end{tabular}

*The read out device for MSOI did not work during the experiment. 